\title{
Jamming in Spin Systems
}

\author{
Anjana Malinge Samarakoon
}

Galle, Sri Lanka

B.Sc. Physics, University of Colombo, Sri Lanka, 2010

\begin{abstract}
A Dissertation presented to the Graduate Faculty of the University of Virginia in Candidacy for the Degree of Doctor of Philosophy
\end{abstract}

Department of Physics

University of Virginia

December 2017 
Dedicated to my family

and

all my teachers. 
"The true sign of intelligence is not knowledge but imagination."

Albert Einstein 


\section{Abstract}

Since the discovery of spin glasses in dilute magnetic systems, their study has been largely focused on understanding randomness and defects as the driving mechanism. The same paradigm has also been applied to explain glassy states found in dense frustrated systems. Recently, however, it has been theoretically suggested that different mechanisms, such as quantum fluctuations and topological features, may induce glassy states in defect-free spin systems, far from the conventional dilute limit. Here we report experimental evidence for the existence of a glassy state, that we call a spin jam, in the vicinity of the clean limit of a frustrated magnet, which shows unconventional spin glass properties, such as the quadratic low-temperature dependence of heatcapacity, a broad elastic neutron scattering peak centred around a non-zero wavevector $(Q)$, and insensitivity to low-concentration of defects. We have mainly studied two isostructural frustrated magnets, $\mathrm{SrCr}_{9 p} \mathrm{Ga}_{12-9 p} \mathrm{O}_{19}(\mathrm{SCGO}(p))$ and $\mathrm{BaCr}_{9 p} \mathrm{Ga}_{12-9 p} \mathrm{O}_{19}(\mathrm{BCGO}(p))$, in which the magnetic $\mathrm{Cr}^{3+}(\mathrm{s}=3 / 2)$ ions form a quasi-two-dimensional (Q2D) triangular system of bi-pyramids, in comparison to several other magnetic glasses ranging from dilute magnetic alloys to threedimensional frustrated magnets.

We performed both bulk susceptibility and neutron scattering experiments on both $\operatorname{SCGO}(p)$ and $\operatorname{BCGO}(p)$ systems as a function of dilution $p$ in the range of $0.4 \lesssim p \lesssim 0.97$. Our bulk susceptibility data exhibit glassy behavior at much lower temperatures $\left(T_{f}\right)$ than the absolute values of the CurieWeiss temperature, $\left|\theta_{C W}\right| \approx-504(2) \mathrm{K}$ for $\operatorname{SCGO}(p=0.968(6))$ and $-695(1) \mathrm{K}$ for $\operatorname{BCGO}(p=$ $0.902(8))$. The frustration index $f=\left|\theta_{C W}\right| / T_{f}$ is as high as 130 for $\operatorname{SCGO}(p=0.968(6))$ and 190 for $\operatorname{BCGO}(p=0.902(8))$ indicating strong frustration. Low-temperature susceptibility measurements of $\operatorname{SCGO}(p) / \mathrm{BCGO}(p)$ reveals glass-like signatures such as zero-field cooled (ZFC) - field cooled (FC) hysteresis and frequency dependent AC susceptibility but the low-temperature magnetic phases found in the dense limit of magnetic ions show distinct properties from conventional spin glasses.

Our inelastic neutron scattering data on $\operatorname{SCGO}(p=0.968(6))$ and $\operatorname{BCGO}(p=0.902(8))$ shows dispersionless magnetic excitations centered at $\hbar \omega=18.6(1) \mathrm{meV}$ and $16.5(1) \mathrm{meV}$ respectively, due to singlet to triplet excitations of spin $\mathrm{s}=3 / 2$ dimers. The spin dimers are formed by $\mathrm{Cr}^{3+}$ ions in two $4 f_{v i}$ layers that lie between $12 k-2 a-12 k$ (kagome-triangle-kagome) trilayers. Moreover, a 
continuum spectrum centered around $Q \approx 1.5 \AA^{-1}$, observed in time-of-flight (TOF) neutron scattering experiments for $\operatorname{SCGO}(p) / \operatorname{BCGO}(p)$ magnets, confirm that the kagome-triangular-kagome trilayer is responsible for low-energy spin dynamics. The imaginary part of dynamical susceptibility $\left(\chi^{\prime \prime}(\omega)\right)$ for $\operatorname{SCGO}(p=0.968(6))$ or $\operatorname{BCGO}(p=0.902(8))$ shows linear dependence at low-energies which is inconsistent with energy independent behavior of dilute spin glasses. Yet, the unconventional dynamics of these magnets can be explained by low temperature Halprin and Saslow (HS) like modes. To investigate the low-energy excitations further, we performed neutron spin echo experiments on the $\operatorname{BCGO}(p=0.902(8))$ sample and observed slowly varying dynamics in the nanosecond time scale below the glass transition temperature which is inconsistent with conventional stretched exponential nature of spin glasses.

To confirm the spin jam nature in $\operatorname{SCGO}(p)$ magnets, we performed TOF neutron scattering experiments as a function of non-magnetic doping. As the nonmagnetic $\mathrm{Ga}^{3+}$ impurity concentration is changed, there are two distinct phases of glassiness: the spin jam which is insensitive to defects, for high magnetic concentration region $(p>0.8)$ and a cluster spin glass for lower magnetic concentration, $(p<0.8)$. This observation indicates that a spin jam is a unique vantage point from which glassy states in frustrated magnets, where the spins are densely packed, can be understood.

Furthermore, we performed a comparative study of three different magnetic glasses using various experimental techniques ranging from bulk susceptibility to inelastic neutron scattering. The systems we studied are a Q2D magnet $\operatorname{BCGO}(p=0.902(8))$, a three-dimensional frustrated magnet $\mathrm{Y}_{2} \mathrm{Mo}_{2} \mathrm{O}_{7}$ and a conventional spin glass $\mathrm{CuMn} 2 \%$. The magnetic elastic order parameter was observed as a function of energy resolution for each sample. The energy resolution dependent ordering temperature could be modeled by Vogel-Fulcher law, indicating glass-like freezing in all the three compounds, yet with different fitting parameters implying distinct glass phases. The magnetic field effects on bulk susceptibility have also been studied for all the three samples and a systematic method to distinguish different magnetic glasses is presented.

The notion of complex energy landscapes underpins the intriguing dynamical behaviors in many systems ranging from polymers to brain activity, to social networks and glass transitions. The spin glass state found in dilute magnetic alloys has been an exceptionally convenient laboratory frame for studying complex dynamics resulting from a hierarchical energy landscape with rugged 
funnels. Here, we show, by a bulk susceptibility and Monte Carlo simulation study, that densely populated frustrated magnets in a spin jam state exhibit much weaker memory effects than spin glasses, and the characteristic properties can be reproduced by a nonhierarchical landscape with a wide and nearly flat but rough bottom. Our memory effect results on $\operatorname{SCGO}(p=0.97)$ and $\operatorname{BCGO}(p$ $=0.96$ ) in comparison to $C u \mathrm{Mn} 2 \%$, illustrate that the memory effects can be used to probe different slow dynamics of glassy materials, hence opening a window to explore their distinct energy landscapes.

Furthermore, we studied memory effects in various magnetic glasses including high-temperature superconductor-related materials, spin-orbit Mott insulators, frustrated magnets, and dilute magnetic alloys to characterize ubiquitous glassiness found in magnetic materials. Here, we show that scaling of magnetic memories with time can be used to classify magnetic glassy materials into two distinct classes. Our bulk magnetization measurements reveal that most densely populated magnets exhibit similar memory behavior characterized by a relaxation exponent of $1-\mathrm{n} \approx 0.6(1)$. This exponent is different from $1-n \approx 1 / 3$ of dilute magnetic alloys that were ascribed to their hierarchical and fractal energy landscape, and is also different from $1-n=1$ of the conventional Debye relaxation expected for a spin solid, a state with long-range order. Furthermore, our systematic study of dilute magnetic alloys with varying magnetic concentration exhibits crossovers among the two glassy states and spin solid.

Finally, we have developed a numerical simulation technique to comprehend neutron scattering experiments on magnetic phases with short-range correlations using Landau-Lifshitz dynamics. Here, we study the spin-S Kitaev model in the classical $(S \rightarrow \infty)$ limit and compare against the dynamical structure factors of the spin-1/2. More interestingly, the low-temperature and lowenergy spectrum of the classical model exhibits a finite energy peak, which is the precursor of the one produced by the Majorana modes of the $S=1 / 2$ model. The classical peak is spectrally narrowed compared to the quantum result and can be explained by magnon excitations within fluctuating one-dimensional manifolds (loops). Hence the difference from the classical limit to the quantum limit can be understood by the fractionalization of magnons propagating in onedimensional manifolds. Moreover, we show that the momentum space distribution of the lowenergy spectral weight of the $S=1 / 2$ model follows the momentum space distribution of zero modes of the classical model. 


\section{Acknowledgements}

First and foremost, I would like to express my sincere gratitude to my Ph.D. advisor Prof. SeungHun Lee for all the support, knowledge, opportunities and motivation he had given me throughout my Ph.D. studies. He has taught me how to plan and perform a successful neutron scattering experiment along with many other academic and non-academic tips. I would also like to thank him for opening up the opportunity to work with Dr. David Alan Tennant at Oak Ridge National Laboratory(ORNL).

In addition, I also take this opportunity to express my sincere appreciation to Dr David Alan Tennant who was my research supervisor at Oak Ridge National Laboratory (ORNL) and Prof. Cristian Batista from the University of Tennessee for all the support, knowledge, opportunities and motivation, they had given me throughout the two years of time I spent at Shull-Wollan Center, ORNL.

Next, I would like to express my deeply felt gratitude to the other members of my thesis committee, Prof. Gia-Wei Chern, Prof. Israel Klich and Prof. Slava Krushkal for their valuable, insightful comments and questions which improved my research in many perspectives. Furthermore, I would like to thank Prof. Despina Louca and Prof. Utpal Chatterjee for being the members of my research committee and giving me useful comments on my research progress timely.

I owe a deep debt of gratitude to Prof. Nilanga Liyanage, who helped me to start my life at the University of Virginia and gave timely advice on diverse of situations throughout my Ph.D. Moreover, I take this opportunity to thank my uncle, Mr. Kesawa Abeysiriwardena who encouraged me to follow my passion for physics and supported in many ways from the beginning.

Furthermore, I also thank Dr. Georg Ehlers for all the knowledge and support, especially regarding Neutron Spin Echo spectroscopy. I also thank Dr. Stephen E. Nagler, Dr. Arnab Banerjee, Dr. Souleymane Omar Diallo and Dr. Piotr A. Zolnierczuk for all the support and fruitful discussions. And I also thank Dr. Ying Wai Li, Dr. Markus Eisenbach and Dr. Hidemaro Suwa for sharing their knowledge on numerical simulations. I specially thank Dr. Ying Wai for proofreading several parts

of my dissertation and giving useful comments and suggestions. Moreover, I appreciate the collaboration with Dr. Yoshimoto Kamiya and Dr. Shang-Shun Zhang, and all the support, given 
by Prof. Yukitoshi Motome to complete the project "Comprehensive study of the dynamics of a classical Kitaev spin liquids" successfully.

I would also like to express my special thanks to Prof. Taku Sato for inviting me to Tohoku University, Japan twice for collaborative research and making necessary arrangements. I should also thank Prof. Taku for giving me unrestricted access to his laboratory and equipment, and his research group for all the support they had given me both inside and outside the school and all the fun we had together.

I must present my sincere thanks to Junjie Yang for making necessary samples for my research and teaching me essential laboratory skills including sample synthesis, single crystal growth, and PPMS measurements. Also, I would like to thank Prof. Haidong Zhou and Ryan Sinclair for providing us necessary samples for research, Prof. Gai-Wei Chern, and Tianran Chen from performing Dynamical Tree Monte-Carlo simulations to reproduce the results from Memory Effect experiments. I must also thank Dr. John R. D. Copley, Dr. Daniel Pajerowski, Dr. Nicholas P. Butch and Dr. Wei Zhou for all their support during the experiments at Disk Chopper Spectrometer (DCS), National Institute of Standards and Technology (NIST), and Dr. Antonio Faraone for all the knowledge given on NSE spectroscopy and all the support provided for the NSE experiments at NCNR. I also thank Dr. Madhusudan Tyagi for me giving me an opportunity to use High Flux Backscattering Spectrometer (HFBS), NIST.

I must call my present and former fellow labmates to mind with gratitude, Dr. Jooseop Lee, Dr. Sachith Dissanayake, Tianran Chen and Depei Zhang for all the useful discussions, support, strength and all the fun we had together.

Last but not least, I must express my heartfelt gratitude to my family and all my teachers. My parents, Asoka Samarakoon and Pushpa Nandani Samarakoon, for their endless love, support, and encouragement throughout my entire life. My lovely wife, Udari Sandamali for understanding and believing me along with love, care, patience, support, and encouragement, she had given me throughout my graduate life. My great-aunt, Chandra Axelsson for supporting throughout my entire college as well as to achieve some of other financial goals in my life, and my other greataunt, Durgadevi Abeysiriwardena who was my $4^{\text {th }}$-grade teacher, for well-wishing and supporting me in many ways since my childhood. Finally, I wish to thank all the other well-wishers and my teachers for their wishes, guidance, and support they have given throughout my life. 


\section{Contents}

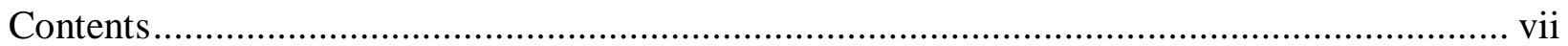

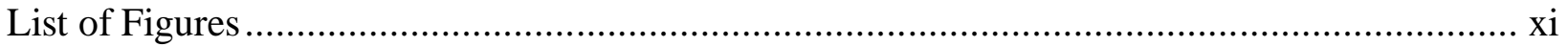

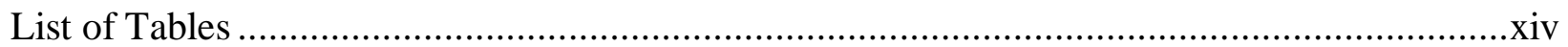

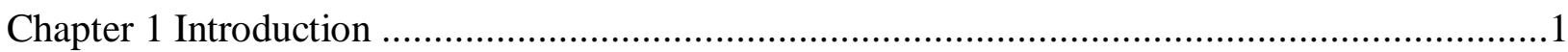

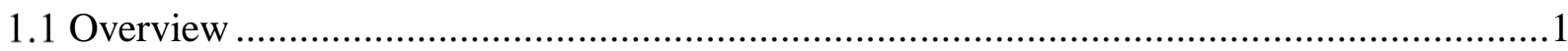

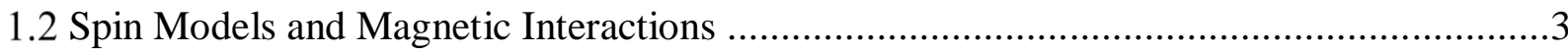

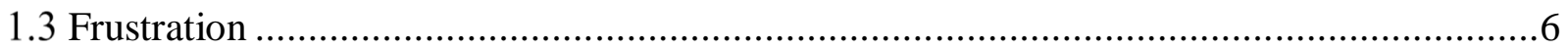

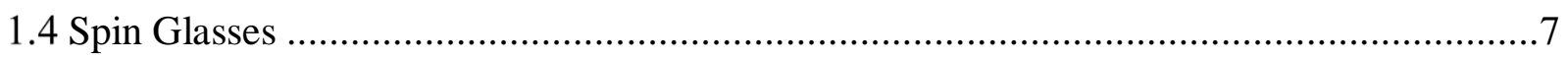

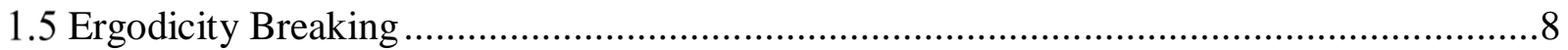

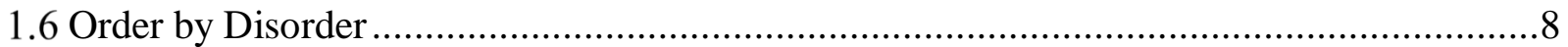

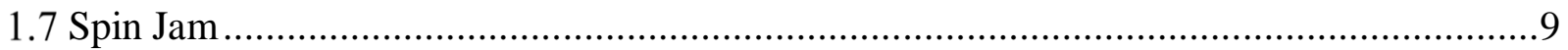

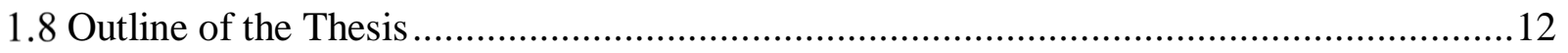

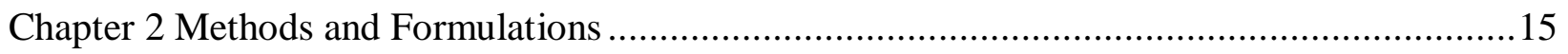

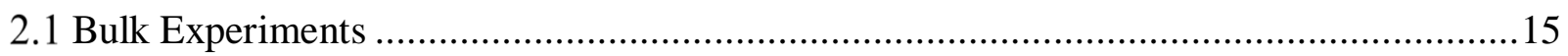

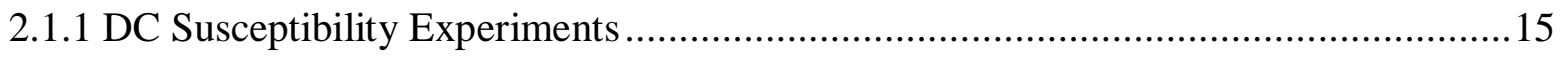

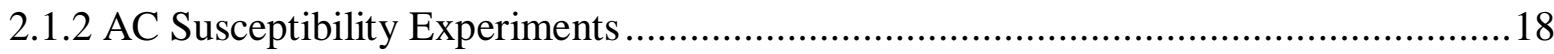

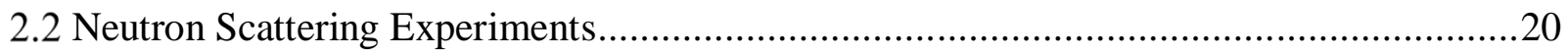

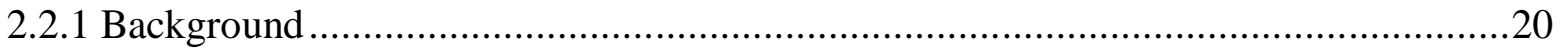

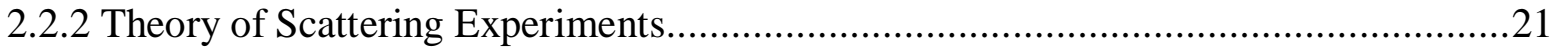

2.2.3 Neutron Backscattering Spectroscopy ......................................................................2

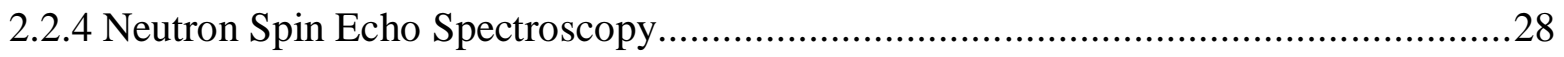

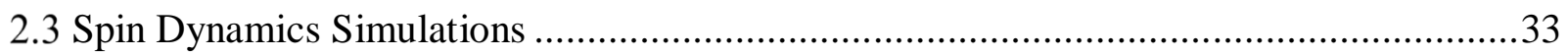


Chapter 3 A Quasi-Two-Dimensional Magnetic System.................................................36

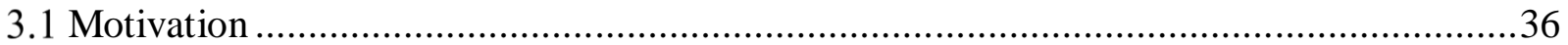

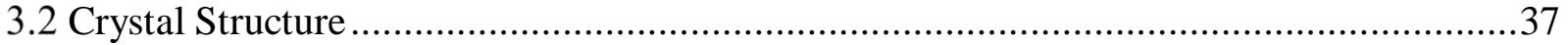

3.3 Bulk Susceptibility Experiments ...................................................................40

3.4 Two-dimensionality of $\operatorname{BCGO}(p=0.902(8))$ and the isolated spin pairs.......................43

3.5 Inelastic Neutron Scattering Spectrum .........................................................46

3.6 Quasi-Elastic Neutron Scattering …............................................................ 48

3.6.1 Neutron Time-of-Flight experiments ....................................................48

3.6.2 Neutron Spin Echo experiments ........................................................... 51

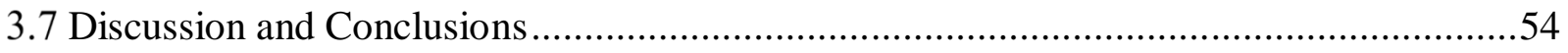

Chapter 4 Crossover from Spin Jam to Spin Glass upon dilution in $\operatorname{SCGO}(p)$........................55

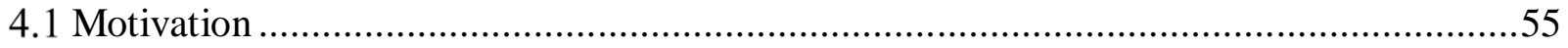

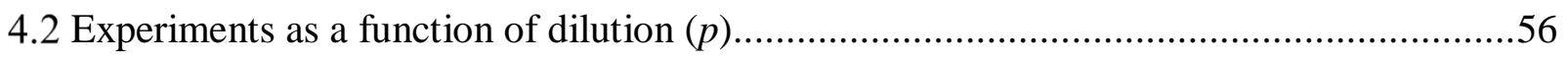

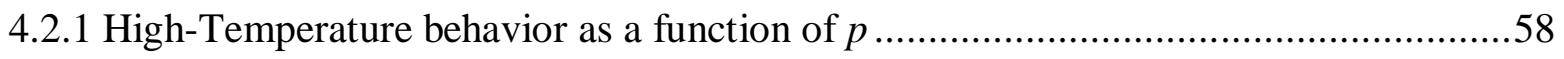

4.2.2 Low-temperature magnetic susceptibility and elastic neutron intensity. ..................59

4.2.3 Quasi-Elastic Neutron Scattering ........................................................6 60

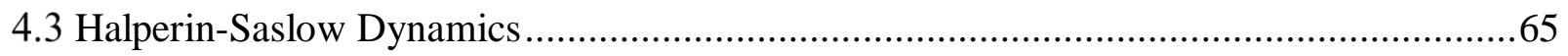

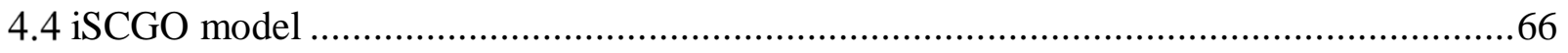

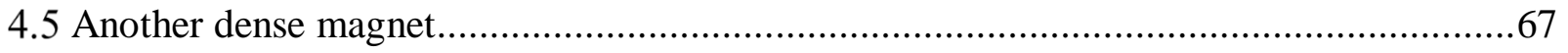

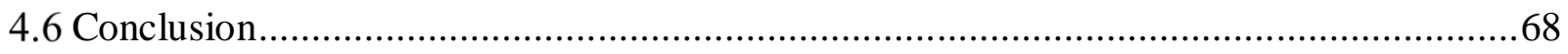

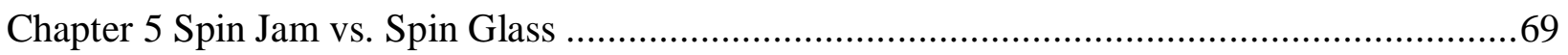

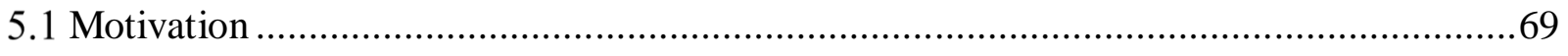

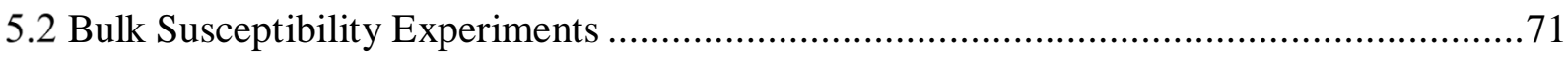

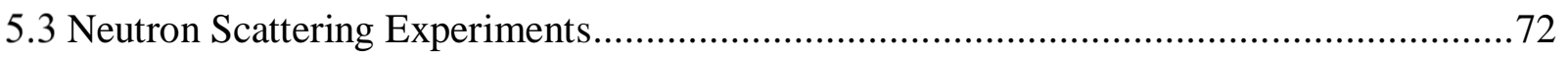

5.3.1 Neutron Spin Echo measurements ...................................................... 72 


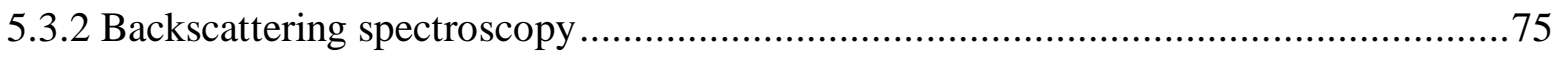

5.4 Spectral Distribution of Relaxation Time ………………….........................................78

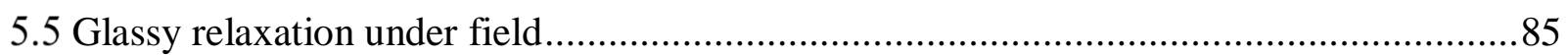

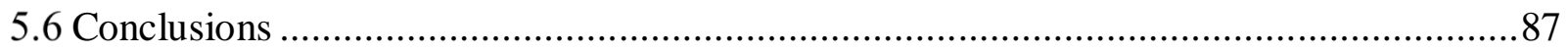

Chapter 6 Aging, Memory, and Nonhierarchical energy landscape of SCGO.............................88

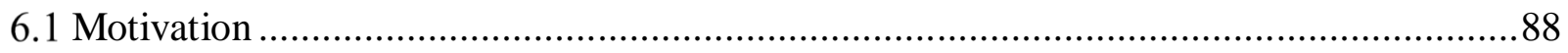

6.2 Aging and Memory Effect Experiments ......................................................................90

6.3 Magnetic Memory of Spin Jam vs Spin Glass ………................................................. 90

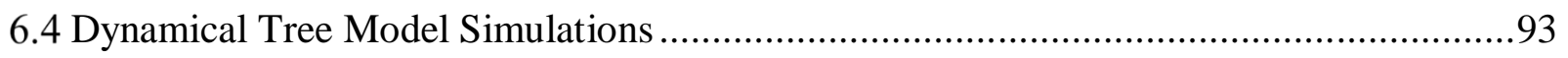

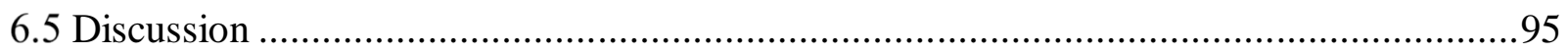

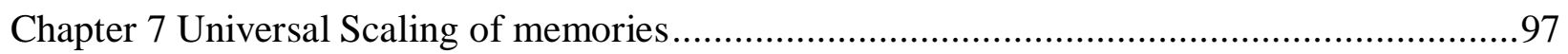

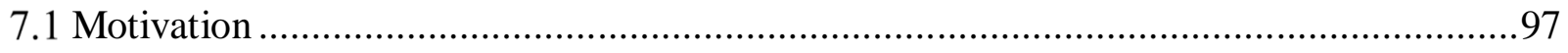

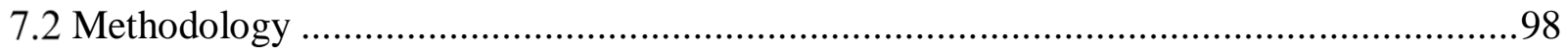

7.3 Universal Memory Behavior .....................................................................................99

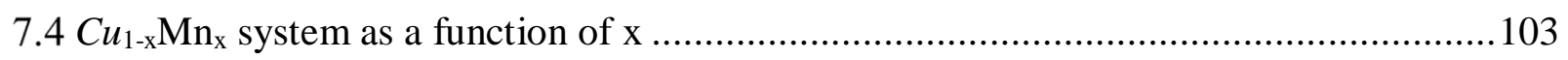

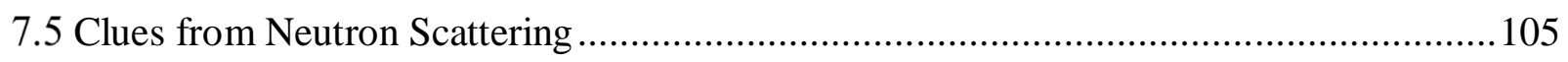

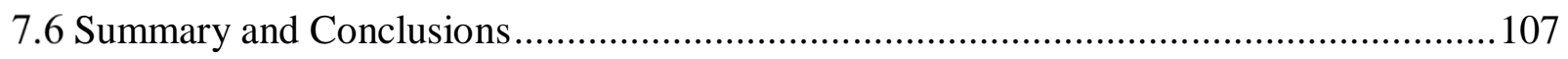

Chapter 8 Comprehensive study of the dynamics of a classical Kitaev spin liquid ...................109

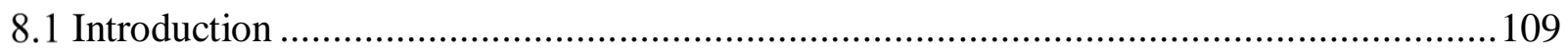

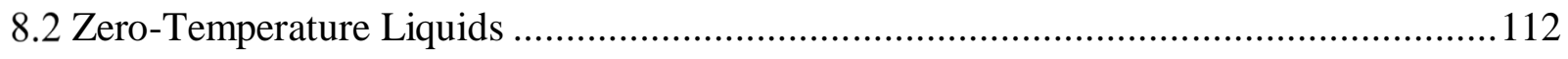

8.3 Finite Temperature Liquids ..................................................................................120

8.4 Kitaev-Heisenberg Model .....................................................................................122

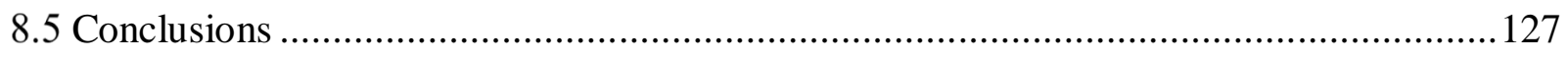

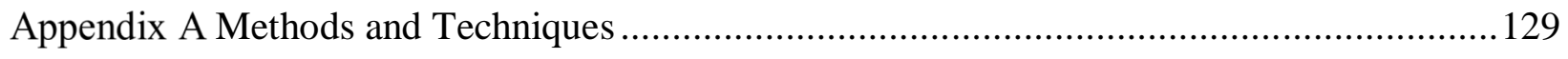

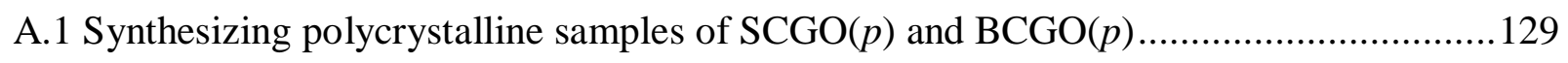




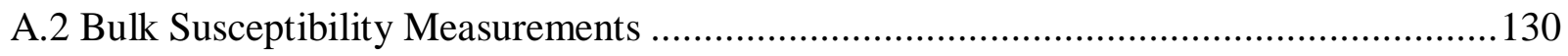

A.3 Crystal Structure determination by Neutron diffraction ..............................................131

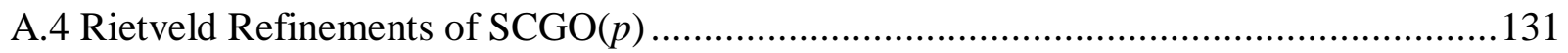

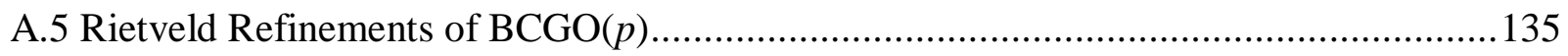

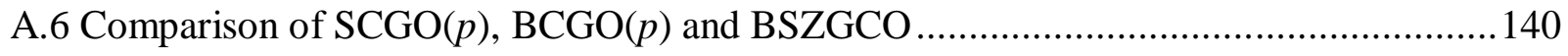

A.7 Time-of-flight Neutron Scattering Measurements.......................................................... 141

A.8 Ultra-Low Field Measurements and Thermoremanent Magnetization.............................141

A.9 $\chi$ and inelastic order parameter as a function of relaxation time .....................................142

Appendix B Spinwave Dispersion for Kagome-Triangular-Kagome Trilayer ...........................145

Appendix C Barrier Tree Models and Monte Carlo Simulations ……......................................146

Appendix D High Temperature susceptibility of $\mathrm{Fe}_{1.02} \mathrm{Se}_{0.15} \mathrm{Te}_{0.85}$ and $\mathrm{La}_{1.96} \mathrm{Sr}_{0.04} \mathrm{CuO}_{4} \ldots \ldots \ldots \ldots .154$

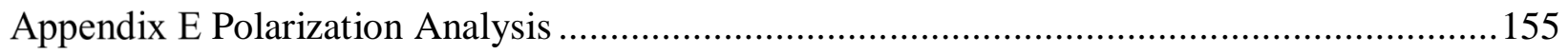

Appendix F Linear Spin Waves for CN-ground States..........................................................156

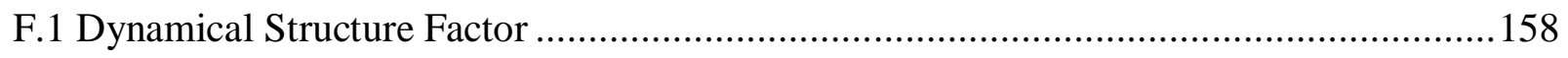

F.2 On-site Dynamical Structure Factor .........................................................................158

F.3 Nearest-neighbor Dynamical Structure Factor................................................................159

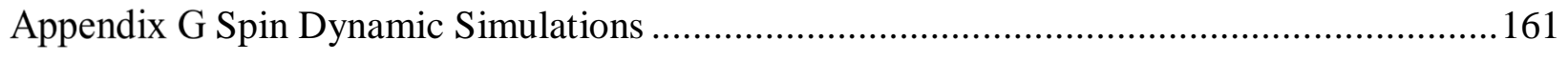

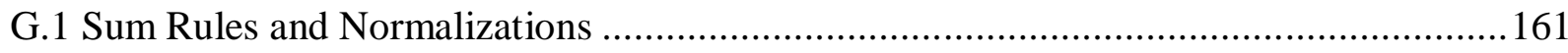

G.2 Simple Heisenberg models in Square Lattice...............................................................164

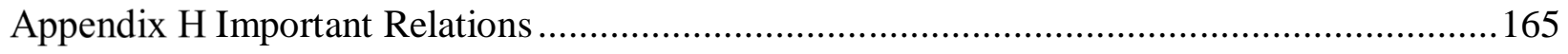

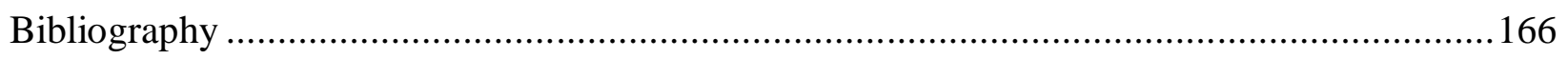




\section{List of Figures}

Figure 1.1 RKKY exchange coupling constant ........................................................

Figure 1.2 An illustration of Superexchange mechanism .............................................5

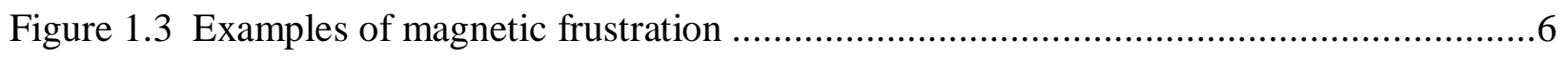

Figure 1.4 The triangular network of bipyramids and its classical ground states ................... 10

Figure 1.5 Rugged energy landscape induced by quantum fluctuations .................................11

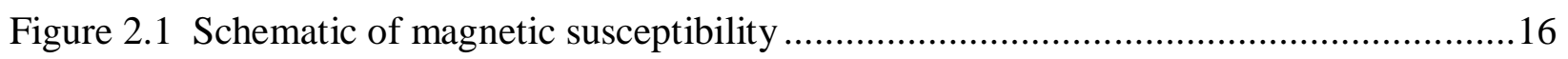

Figure 2.2 Low-T susceptibility measurements of two Spin Glasses: $C u \mathrm{Mn} 2 \%$ and $A u \mathrm{Fe} 2 \% \ldots 17$

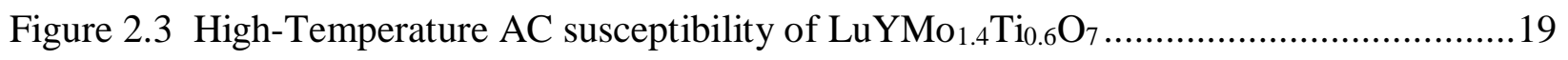

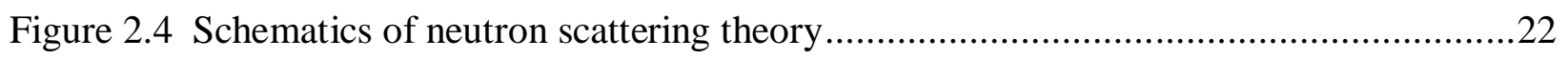

Figure 2.5 Schematics of a Neutron Time-of-Flight spectrometer .......................................24

Figure 2.6 A schematic of a neutron backscattering spectrometer ...................................27

Figure 2.7 Generic setup of a reactor-based neutron spin echo spectrometer........................29

Figure 2.8 Neutron Spin Echo Order Parameter on $\mathrm{CuMn2 \%} \mathrm{.............................................31}$

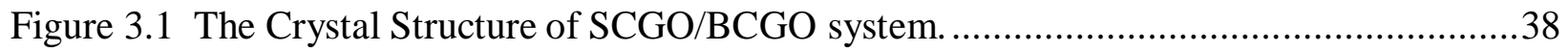

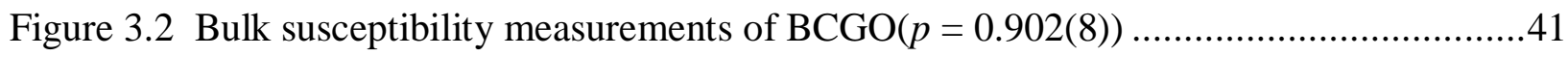

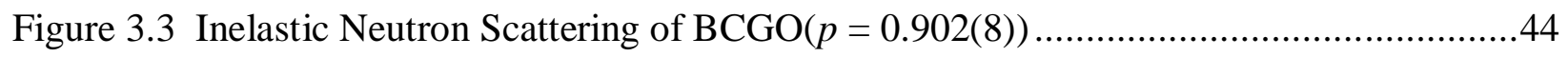

Figure 3.4 Temperature dependence of dimer excitation in $\operatorname{BCGO}(p=0.902(8)) \ldots \ldots \ldots \ldots \ldots . . . . .45$

Figure 3.5 Entire neutron scattering spectrum of BCGO and SCGO systems ........................47

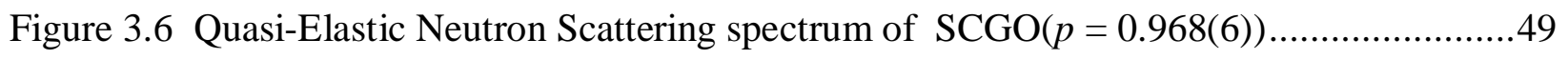

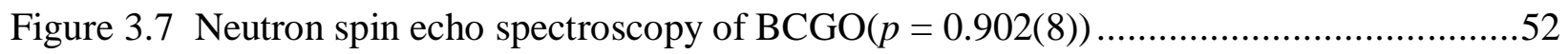

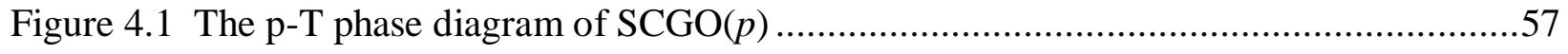

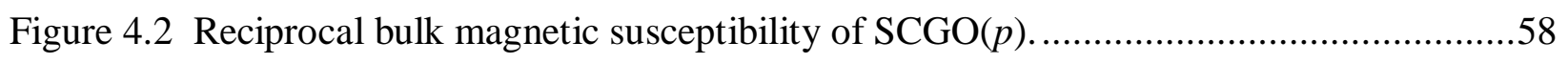

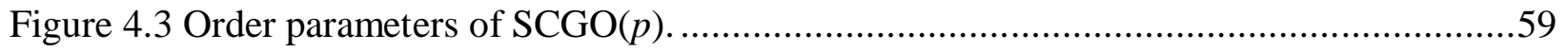

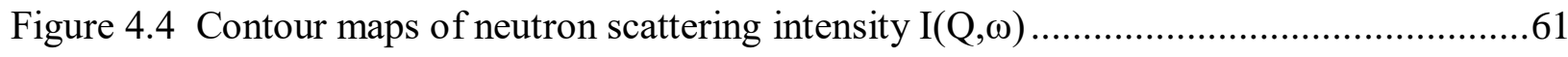

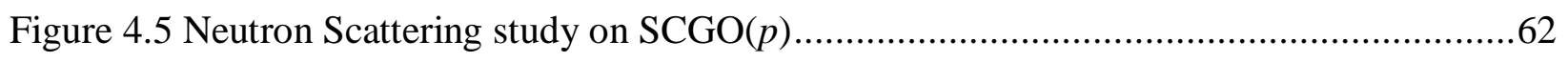

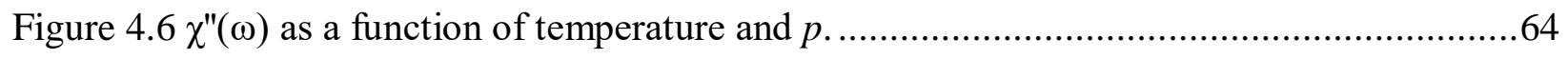

Figure 5.1 Bulk Susceptibility of $C u \mathrm{Mn} 2 \%, \operatorname{BCGO}(p=0.902(8))$ and $\mathrm{Y}_{2} \mathrm{Mo}_{2} \mathrm{O}_{7} \ldots \ldots \ldots \ldots \ldots \ldots . . \ldots 1$

Figure 5.2 Magnetic order-parameters from Neutron Spin Echo .........................................73

Figure 5.3 Neutron Backscattering experiments of $\mathrm{BCGO}(p=0.9), \mathrm{Y}_{2} \mathrm{Mo}_{2} \mathrm{O}_{7}$ and $C u \mathrm{Mn} 2 \% \ldots \ldots .75$ 


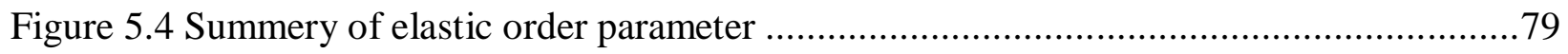

Figure 5.5 Freezing behaviors and relaxation time distributions of three magnetic glasses .........81

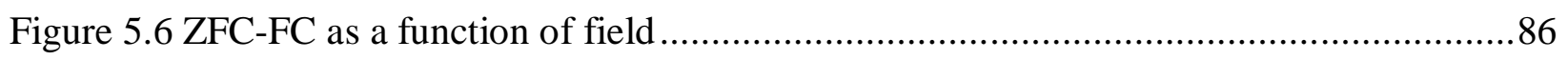

Figure 6.1 Schematic energy landscapes of spin glass and spin jam................................. 89

Figure 6.2 Magnetic memory as a function of $\mathrm{t}_{\mathrm{w}}$ for SCGO, BCGO and $C u \mathrm{Mn} 2 \%$................91

Figure 6.3 Magnetic memory as a function of Tw for SCGO, BCGO and $C u \mathrm{Mn} 2 \% \ldots \ldots \ldots \ldots \ldots . . . . .92$

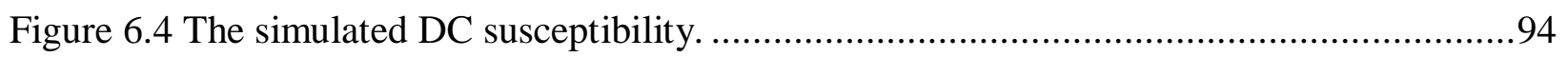

Figure 7.1 Memory Effect as a function of waiting time. ........................................... 100

Figure 7.2 Summarizing the memory effect............................................................ 101

Figure 7.3 Temperature Dependence of memory effect.............................................. 103

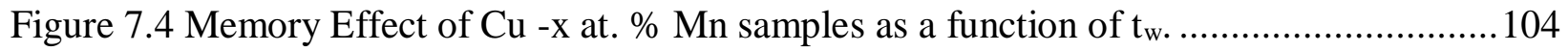

Figure 7.5 Neutron scattering measurements of NITO and $C u \mathrm{Mn} 2 \%$..............................106

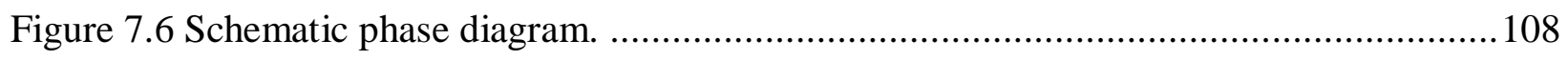

Figure 8.1 The Kitaev-Heisenberg model. ...........................................................111

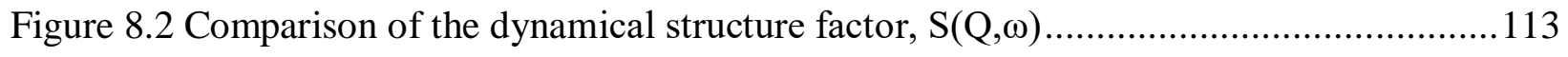

Figure 8.3 Temperature dependences of spin-spin correlations. ....................................114

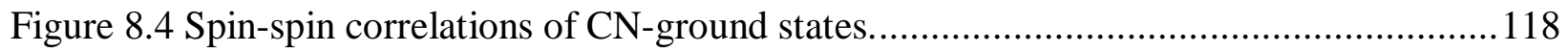

Figure 8.5 Temperature evolution of $\mathrm{S}(\mathrm{Q}, \omega)$ of AFM and FM Kitaev models.......................121

Figure 8.6 Schematic phase diagram of the classical K-H model for $(\mathrm{K}>0)$........................ 123

Figure $8.7 \mathrm{~S}(\mathrm{Q}, \omega)$ as a function of FM Heisenberg exchange $(\mathrm{J}<0)$...............................124

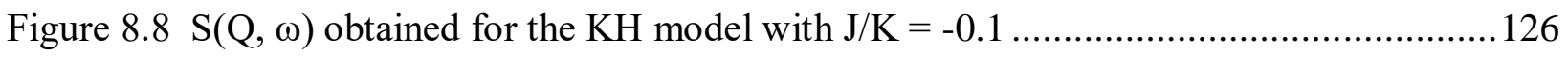




\section{Figures of Appendix}

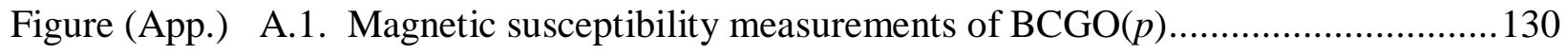

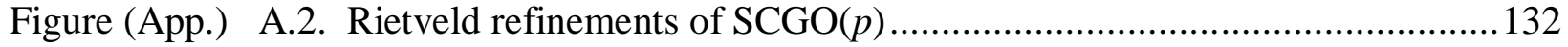

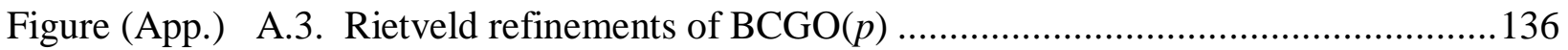

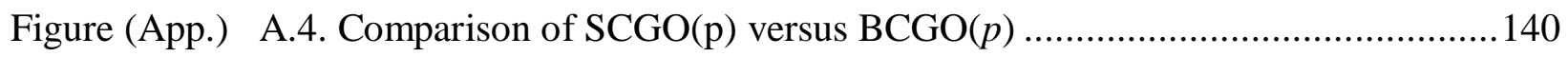

Figure (App.) A.5. Susceptibility and inelastic order parameter for $\operatorname{BCGO}(p=0.902(8)) \ldots . .142$

Figure (App.) A.6. Susceptibility and inelastic order parameter for $C u \mathrm{Mn} 2 \%$.................... 143

Figure (App.) A.7. Susceptibility and inelastic order parameter for $\mathrm{Y}_{2} \mathrm{Mo}_{2} \mathrm{O}_{7} \ldots \ldots \ldots \ldots \ldots \ldots . . . . . . .144$

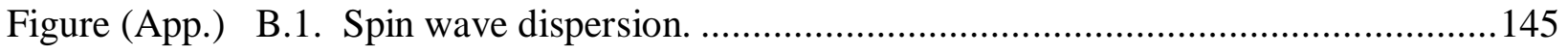

Figure (App.) C.1. Schematic diagram showing a Monte Carlo step in our dynamic barrier tree

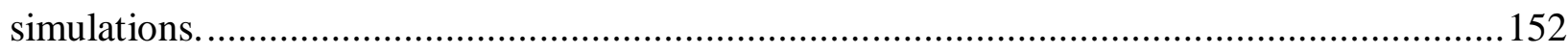

Figure (App.) C.2. Simulated Magnetization as a function of temperature. ........................... 153

Figure (App.) D.1. High-Temperature bulk susceptibility of LSCO and FST samples..........154

Figure (App.) G.1. Dispersion relations of simple Heisenberg square lattices ...................... 164 


\section{List of Tables}

Table 2-1 Comparison of Cold, Thermal and Hot neutrons.

.21

Table 3-1 Comparison of lattice constants and bond distances between $\mathrm{Cr}^{3+}$ ions in SCGO/BCGO

Table 3-2 Fitting parameters of the inelastic spectrum of $\operatorname{BCGO}(p)$ and $\operatorname{SCGO}(p)$ samples......48

Table 5-1 Fitting parameters of Vogal-Fulcher law for $\mathrm{BCGO}(p), \mathrm{Y}_{2} \mathrm{Mo}_{2} \mathrm{O}_{7}$ and $C u \mathrm{Mn} 2 \% \ldots \ldots .83$

Table 5-2 Comparison of critical field extracted from ZFC-FC magnetization experiments .......86

\section{Tables of Appendix}

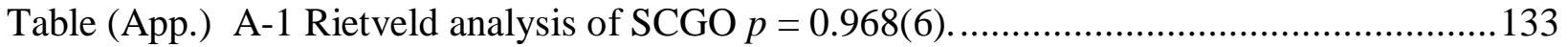

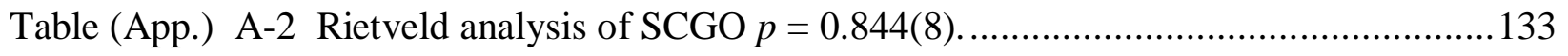

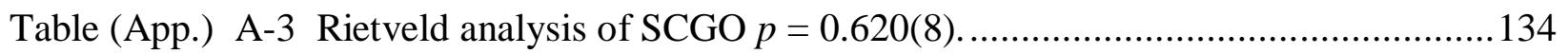

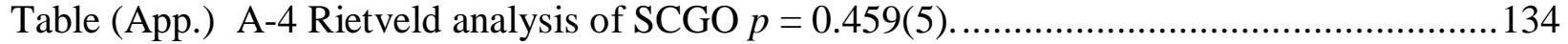

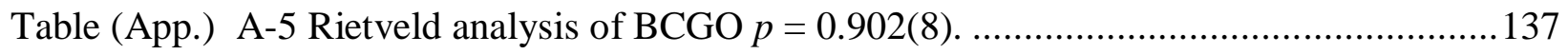

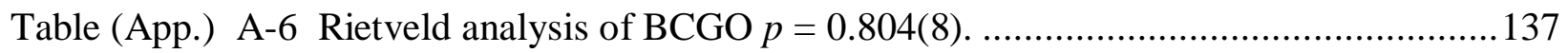

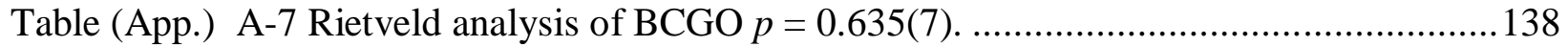

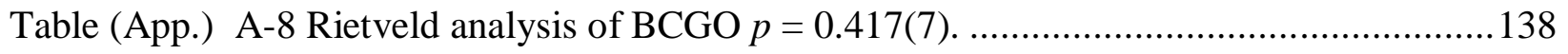

Table (App.) A-9 Summery of $\mathrm{T}_{\mathrm{DC}}$ and $\theta_{\mathrm{cw}}$ for $\operatorname{BCGO}(p), \operatorname{SCGO}(p)$ and $\operatorname{BSZGCO} \ldots \ldots \ldots \ldots . . . .140$ 


\section{Physical Constants}

Speed of Light

Plank Constant

Reduced Plank Constant

Mass of Electron

Mass of Neutron

Bohr Magneton

Boltzmann constant

$$
\begin{aligned}
c & =2.99792458 \times 10^{8} \mathrm{~ms}^{-2} \\
h & =6.626070040(81) \times 10^{-34} \mathrm{~J} \mathrm{~s} \\
& =4.135667662(25) \times 10^{-3} \mathrm{meV} \mathrm{ns}^{-34} \mathrm{~J} \mathrm{rad}^{-1} \\
\hbar & =1.054571800(13) \times 10^{-3} \\
& =6.582119514(40) \times 10^{-4} \mathrm{meV} \mathrm{ns} \mathrm{rad}^{-1} \\
m_{e} & =9.10938356(11) \times 10^{-31} \mathrm{~kg} \\
m_{n} & =1.674927471(21) \times 10^{-27} \mathrm{~kg} \\
\mu_{B}\left(=\frac{e \hbar}{2 m_{e}}\right) & =9.274009994(57) \times 10^{-24} \mathrm{~J} \mathrm{~T}^{-1} \\
& =5.7883818012(26) \times 10^{-2} \approx 0.059 \mathrm{meV} \mathrm{T}^{-1} \\
k_{B} & =1.38064852(79) \times 10^{-23} \mathrm{~J} \mathrm{~K}^{-1} \\
& =8.6173324(78) \times 10^{-2} \approx 0.086 \mathrm{meV} \mathrm{K}{ }^{-1}
\end{aligned}
$$




\section{Symbols}

$\begin{array}{lll}E=\hbar \omega & \text { Energy Transfer } & \mathrm{meV} \\ \hbar \vec{Q} & \text { Momentum Transfer } & \mathrm{kg} \mathrm{ms}^{-1} \\ \Omega & \text { Solid Angle } & \mathrm{sr} \text { (Steradian : dimensionless) } \\ \sigma & \text { Scattering Cross Section } & \mathrm{b}\left(1 \mathrm{barn}=10^{-28} \mathrm{~m}^{2}\right) \\ \frac{d^{2} \sigma}{d \Omega d E} & \text { Differential Scattering Cross Section } & \mathrm{b} \mathrm{sr}^{-1} \mathrm{eV}^{-1} \\ S(\vec{Q}, \omega) & \text { Scattering Function } & \mathrm{b}^{-1} \mathrm{sr}^{-1} \mathrm{eV}^{-1} \\ I(\vec{Q}, t) & \text { Intermediate Scattering Function } & \mathrm{b}^{-1} \mathrm{sr}^{-1} \mathrm{eV}^{-1} \\ H & \text { Magnetic Field } & \mathrm{Oe}(\mathrm{Oersted})^{2}\end{array}$




\section{Chapter 1}

\section{Introduction}

\subsection{Overview}

Jamming is an intriguing physical process, happening everywhere around us, in which the liquidity of a system is suppressed and freezes into a disordered phase, for example from soap foams to traffic jams. In the case of solid materials, such a state might look like a solid (glass) in which constituents are frozen in time and space, but the real question is: Is it truly a solid (glass)? Or does it belong to a new class of properties?

Cates et al. argue that jammed states of matter belong to a new category of 'fragile matter' [1]. Usually, a jammed state can be easily achieved by a dense particle system by applying external stress fields, and the jammed state will be relieved upon slight alteration of the stress field [2]. For example, in the case of foam, atmospheric pressure acts as an isotropic stress field to the densely packed soap bubbles coming from a spray can and freezes them into a single unit with a definite shape, but a small shear force can easily deform the shape of foam. From the viewpoint of thermodynamics, the system is trapped in a metastable state of a nearly degenerated rugged energy landscape where the kinematics of the system is confined in the phase space. However, an additional shear force large enough to overcome the nearby potential barriers can move the system into a different metastable state. In other words, a jammed system will remain the same as long as the external perturbations are not strong enough to overcome the barrier potential. As A. J. Liu and S. R. Nagel have pointed out the class of jammed materials may actually be very broad and diverse [2]. Similar to jammed states found in granular systems, it might be possible to 
find jammed states in magnetic systems in which ions with effective magnetic moments (spins) arranged in crystallographically ordered lattices.

Ever since ferromagnetism was first discovered in Lodestones, magnetism became an intriguing subject of research in Science and Technology. In ferromagnets, antiferromagnets or ferrimagnets, the spins will freeze upon cooling into long-range ordered states below a certain temperature. Subsequently, in the search of magnetism, magnetic phases were found in dilute magnetic alloys such as $A u C o$ or $C u M n$ in which spins freeze in a disordered fashion at low temperatures, named spin glasses in analogous to the atomic glasses. Since then, the search for new magnetic phases and characterization of their properties has become a major scientific branch of research in Condensed Matter Physics [3]. During the last decades, many novel magnetic phases have been found including spin glasses, spin liquids, super-paramagnets, cooperative paramagnets and chiral magnets. Moreover, glass-like magnetic phases with intriguing properties have been identified in many different materials ranging from geometrically frustrated magnets to high-temperature superconductors.

Frustration and randomness have been identified as the freezing mechanism of spin glasses found in dilute magnetic alloys in which magnetic ions are embedded in the sea of conduction electrons of a nonmagnetic host. Glassiness found in the non-dilute limit of geometrically frustrated magnets has proven to be a difficult problem in Condensed Matter Physics. Recently, a mechanism in which glass-like freezing is obtained by quantum fluctuation was proposed and applied to the case of a two-dimensional lattice of KagomeTriangular-Kagome trilayers. [4,5] Here, the energy landscape of quantum fluctuation induced frozen states is discussed as a non-hierarchical rugged energy landscape rather than a hierarchical landscape proposed for conventional spin glasses and hence the frozen state is identified as a spin jammed state. [5] In this thesis, we explore the scenario of spin jam states, their origins, and contradictory properties compared to conventional spin glasses. 


\subsection{Spin Models and Magnetic Interactions}

When a magnetic system is comprised of magnetic ions with localized unpaired electrons, such a magnetic system can be explained by an effective mathematical model in which the total energy of the system is explained by localized magnetic moments (spins) rather than the full electronic description of the system. In the classical limit $(S \rightarrow \infty)$, the Hamiltonian of a generic system can be modeled as a combination of exchange interactions, crystalline anisotropies, the external magnetic field, dipole-dipole interactions and many other.

A simple prototypical Hamiltonian can be written as,

$$
H=-\sum_{\{i . j\}} J_{i j} S_{i} \cdot S_{j}-D \sum_{i}\left(\hat{n}_{i} \cdot S_{i}\right)^{2}-h \sum_{i} \hat{n}_{h} \cdot S_{i}-w \sum_{i<j} \frac{3\left(S_{i} \cdot e_{i j}\right)\left(e_{i j} \cdot S_{j}\right)-S_{i} \cdot S_{j}}{r_{i j}^{3}}
$$

The first term is the exchange interaction term and $J_{i j}$ is the interaction energy between two spins $\left(S_{i}\right)$. When $J>0$, this term yields ferromagnetic order for any lattice while antiferromagnetic order occurs in the case of $J<0$ for an unfrustrated lattice. (Frustration will be discussed in the next section) The second term represents the crystalline anisotropy in which $D$ is the anisotropic energy and $\hat{n}_{i}$ is the single ion anisotropy direction of the $i^{\text {th }}$ spin, and the third term is for the Zeeman energy of the system in which $h$ and $\hat{n}_{h}$ are the external magnetic field and its direction respectively. The fourth term is the dipole-dipole coupling of the magnetic moments and $r_{i j}$ is the normalized distances between moments $i$ and $j$, and the $e_{i j}$ is unit vectors in the direction of $r_{i j}$ and $w$ is the interaction energy. Moreover, a microscopic Hamiltonian might also have more complex interactions like asymmetric Dzyaloshinskii-Moriya (DM) interactions, as a consequence of strong spinorbit coupling present in a magnetic system, but the following discussion will be limited only to Magnetic-exchange interactions.

Magnetic-exchange interactions come in two flavors in which spins align either parallel (Ferromagnetic) or antiparallel (Antiferromagnetic) to minimize coulomb repulsion between magnetic ions. Moreover, there are several different exchange mechanisms such as Direct exchange, Indirect exchange, Superexchange, etc. 
Direct exchange interaction is a consequence of direct overlap of the electronic wave function of two magnetic ions, and the flavor of the interaction is determined by Pauli exclusion principle. To review Pauli exclusion principle, the total wave function of two identical fermions (particles with 1/2-integer spin) has to be asymmetric. For example, in a simple case of two Hydrogen ions, constructive interference between two wave functions are energetically favored, and hence anti-parallel spin arrangement is selected by Pauli exclusion principle. Moreover, the interactions by direct exchange mechanism are strong but short-ranged.

Indirect exchange interaction couples two spins via itinerant electrons in the medium between two magnetic ions. Usually, such exchange interactions are expected when magnetic ions embedded in a conducting host lattice and named as Ruderman - Kittel Kasuya - Yoshida (RKKY) interactions. The RKKY interactions are long-ranged, and the sign of the interactions oscillates with the distance between two ions. (see Figure 1.1)

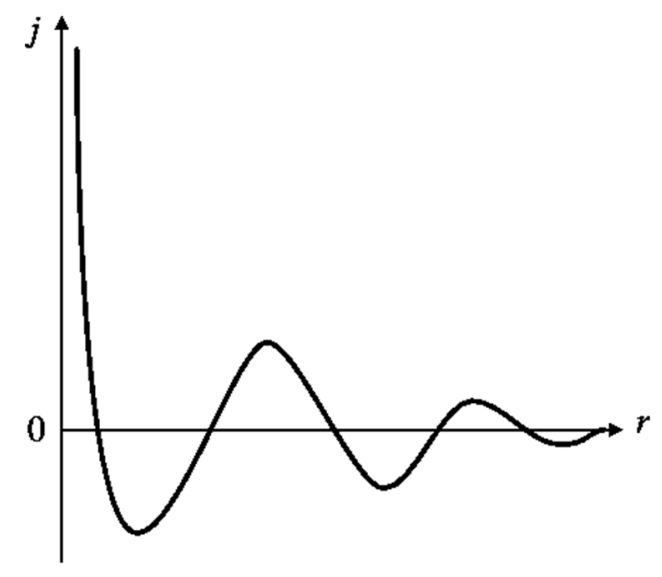

Figure 1.1 RKKY exchange coupling constant

Variation of the indirect exchange coupling constant, $j$, of a free electron gas in the neighborhood of a point magnetic moment at the origin $r=0$. [6]

A magnetic ion induces a spin polarization of the nearby conduction electrons, and spin polarization is transferred through many itinerant electrons to another magnetic ion by introducing an effective exchange coupling between two ions $\left(S_{i}\right.$ and $\left.S_{j}\right)$ described according to,

$$
J(r)=6 \pi Z J^{2} N\left(E_{F}\right)\left[\frac{\sin \left(2 k_{F} r\right)}{\left(2 k_{F} r\right)^{4}}-\frac{\cos \left(2 k_{F} r\right)}{\left(2 k_{F} r\right)^{3}}\right]
$$


where $Z$ is the number of conduction electrons per atom, $J$ is the s-d exchange constant, $N\left(E_{F}\right)$ is the density of state at the Fermi level, $k_{F}$ is the Fermi momentum and $r$ is the distance between two impurities.

Superexchange describes the coupling between two magnetic ions too far apart to be connected by direct exchange but coupled over a relatively long distance through nonmagnetic atoms. This is the primary exchange mechanism in magnetic insulators and occurs covalent mixing of the $\mathrm{p}$ and $\mathrm{d}$ (or $\mathrm{f}$ ) wave functions. The polarization of two electrons in a p-shell must be opposite in direction, and as shown in Figure 1.2, when two magnetic cations are connected through such a ligand, it generally causes antiferromagnetic coupling. Superexchange interactions are stronger and long-ranged compared to directexchange.

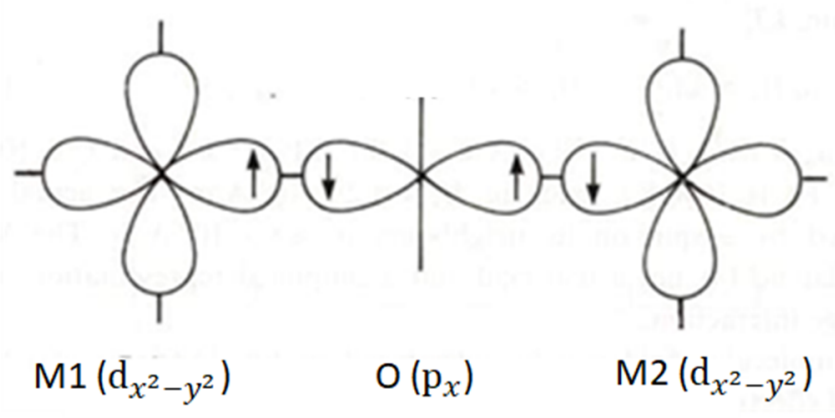

Figure 1.2 An illustration of Superexchange mechanism

M1 and M2 are two transition metal ions coupled antiferromagnetically through an oxygen ion $\left(\mathrm{O}^{2-}\right)$.

Magnetic Anisotropy defines the directional dependence (spin degree of freedom) of magnetic moments and plays an important role when studying possible phase transitions and magnetic properties of different phases, associated with a microscopic Hamiltonian. The simplest examples of anisotropy are Ising models in which all the spins are constrained to align along a single direction (easy axis) either parallel or anti-parallel, XY model in which all the spins are constrained to have any direction on a plane (easy plane) and Heisenberg model in which spin directions are fully isotropic. 


\subsection{Frustration}

When spins are arranged in a square motif with antiferromagnetic nearest neighbor couplings, we can easily find a configuration to satisfy all the magnetic interactions as shown in Figure 1.3 (a). But in some cases, it is impossible to find a configuration to satisfy all the magnetic interactions. This is named as frustration, and it mainly happens in two different ways, either by competing for ferromagnetic and antiferromagnetic couplings or when the lattice is geometrically frustrated.

(a)

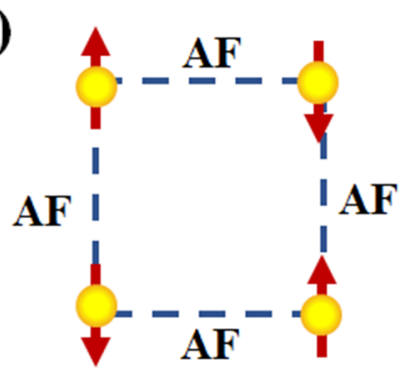

(c)

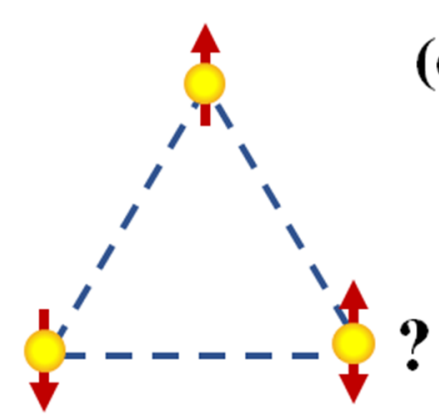

(b)

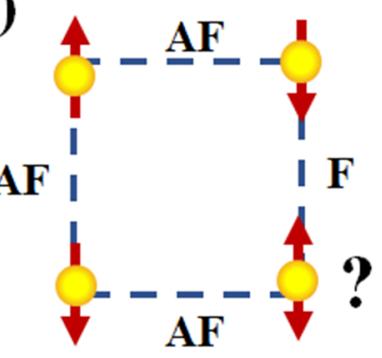

(d)

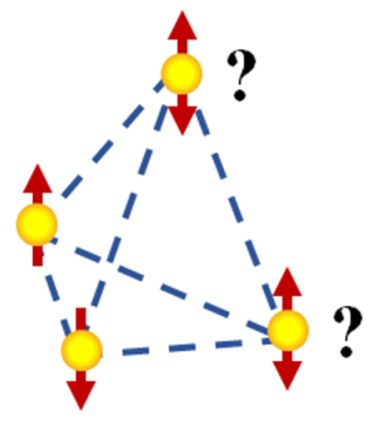

Figure 1.3 Examples of magnetic frustration

(a) Unfrustrated square lattice with antiferromagnetic(AF) interactions. (b) Frustrated square lattice with mixed interactions of antiferromagnetic (AF) and ferromagnetic (F). (c) Frustration from antiferromagnetic triangular motif (d) Frustrated antiferromagnetic tetrahedral arrangement of spins.

As shown in Figure 1.3 (b), when we replace one of the AF-coupling by F-coupling, the lattice becomes frustrated, and this is an example of frustration by mixed interactions. Notice that the ground state degeneracy of the system is two-fold for Ising spins while it is infinite for XY or Heisenberg spin systems. Moreover, when spins arranged in an antiferromagnetically coupled triangular motif (a triangle for a 2D lattice and a tetrahedron for a 3D lattice), all the magnetic interactions cannot be satisfied simultaneously (see Figure 1.3 (c) and (d)) and it is called Geometrical Frustration. 
When frustration appears in a magnetic system, it will either freeze into a single configuration below a certain temperature much lower than the average interaction energy scale of the system or stay in a liquid-like state down to near absolute zero. However, such a system has an extensive ground state degeneracy and can have either liquid-like flat energy landscape or a complex, rugged energy landscape, unlike simple energy landscapes with a single minimum found in unfrustrated systems. In some special cases, the frustration might be lifted by extra perturbations such as crystallographic distortions (Jahn-Teller distortion) or quantum fluctuation.

\subsection{Spin Glasses}

The term "spin glass" was first coined in 1970 to name the low-temperature disordered phase of dilute magnetic alloys such as $A u \mathrm{Co}$ or $C u \mathrm{Mn}$ in which magnetic impurities are doped in a non-magnetic conducting material (written in italics). Here, the magnetic impurities interact with each other via RKKY interactions induced by the electron sea of the conducting material. Numerous experiments and theoretical developments have been performed over last four decades, and frustration and disorder of a magnetic system have been understood as the key ingredients of spin glass freezing. [7]

Unlike in conventional magnets, spin glass materials do not freeze into long-range ordered phases down to lowest temperatures, instead, they undergo phase transitions to short-range ordered phases. Even though short-range spatial correlations can be directly observed from elastic neutron scattering experiments, many other intriguing properties of spin glasses can be found via other experimental techniques such as specific heat, bulk susceptibility, Mössbauer experiments, muon spin echo experiments, quasi-elastic neutron scattering experiments, etc. Among many spin glass signatures, the linear dependence of specific heat is one of the first anomalous behaviors observed in diluted magnetic alloys. Furthermore, the sharp cusp of susceptibility at low-temperatures, measured under low-field conditions, and divergence of non-linear susceptibility, implying a true phase transition at a finite temperature are further experimental signatures of a spin glass. Some other signatures from bulk susceptibility and neutron scattering experiments will be discussed later in this Thesis, and a detailed discussion can be found in ref. [7]. 


\subsection{Ergodicity Breaking}

When there are many metastable states in the energy landscape of a magnetic system, it will get trapped in one of those states below certain temperature with restricted access to other energetically equivalent regions of phase space. This is a property of ruggedness in an energy landscape, and this phenomenon is named as Ergodicity-Breaking. [8] The magnetic irreversibility observed in direct-current (DC) susceptibility under zero-field cooled (ZFC) and field-cooled (FC) conditions, is direct evidence of ergodicity-breaking in a magnetic material. (More information can be found in Section 2.1.1 ) Usually, the magnetic irreversibility will be observed below a certain temperature which can be seen as the highest energy barrier which separates regions in the phase space. Moreover, this behavior can be observed in many different magnetic systems such as magnetic glasses [7], superparamagnets $[9,10]$ or even in long-range ordered systems with multiple domains [11].

Non-ergodic behavior from DC susceptibility is used as a primary test to identify magnetic glasses. Additional characterizations of a glass phase include ascertaining the glasses transition temperature defined by the cusp of ZFC susceptibility $\left(T_{D C}\right)$, and the irreversibility temperature where ZFC-FC splitting occurs $\left(T_{\text {irr }}\right)$. In most of the conventional spin glasses, irreversibility happens below the glass transition temperature, [7] but in some magnetic glass-like materials, the magnetic irreversibility occurs at temperatures higher than $T_{D C}[11]$.

\subsection{Order by Disorder}

When spins are coupled antiferromagnetically and arranged in a geometrically frustrated lattice such as a Kagome lattice, the classical ground state of the system will be macroscopically degenerate. Here, the ground state manifold is generally flat, and configurations are connected continuously via zero-modes. Thus, such a magnetic system usually stays in a liquid-like state down to near absolute zero, upon cooling. But in some systems, thermal fluctuation or quantum fluctuations tends to favour certain configurations over others by breaking the degeneracy of the system and hence the system can have an ordering below a certain temperature. This mechanism is called order-by-disorder. [12] In 
the case of thermal fluctuations, different configurations have different entropic weights, and the configurations with the lowest free energy are favoured. But, when the quantum effects are stronger in a particular magnetic system, the quantum zero-point energy might be different from one configuration to the other and hence select an ordered state with lowest ground state energy. [5,13] Moreover, in Heisenberg pyrochlore antiferromagnets, collinear configurations are usually favoured by thermal/quantum fluctuations [14] while coplanar configurations are favoured in Heisenberg-Kagome antiferromagnets [13,15].

\subsection{Spin Jam}

Spin jam is a magnetic glass phase purely induced by quantum fluctuation from a geometrically frustrated lattice whose ground state manifold is extensively degenerated and flat at mean-field level. In other words, quantum corrections lift the classical degeneracy into a set of aperiodic spin configurations forming local minima in a rugged energy landscape. This theory was first proposed by a simple model in which Heisenberg spin-3/2 moments arranged in a quasi-two-dimensional network of Kagome-Triangular-Kagome trilayers (KTK) with a simple nearest neighbour-spin Hamiltonian $H=J \sum_{N N} S_{i} . S_{j}$. (see Figure $1.4(\mathrm{a}))$

As shown in Figure 1.4 (a), the magnetic lattice is comprised of corner-sharing tetrahedra (Bipyramids / Pyrochlore) and linking triangles that connect bipyramids in Kagome layers. The ground state of the magnetic lattice requires zero net magnetization in each tetrahedron and linking triangles. Triangles usually prefer $120^{\circ}$ configurations while a tetrahedron can adopt any one of an infinite number of possibilities which includes collinear, coplanar or non-coplanar configurations. [4] Since collinear configurations are usually favoured by thermal/quantum fluctuations in Pyrochlore lattices [14], the collinear configurations are an important subset of the KTK lattice. Thus, a special set of ground state configurations can be found as collinear bipyramids arranged in $\sqrt{3} \times \sqrt{3}$ order and named as locally collinear (LC) states. To be precise, there are 18 different collinear states of bipyramids and nine of them are shown in Figure 1.4 (b) using binary signs which represents parallel $(+)$ or anti-parallel (-) direction of each spin with respect to the direction defined by the color of the bipyramid. The other nine configurations can be obtained by sign inversion. [4] 


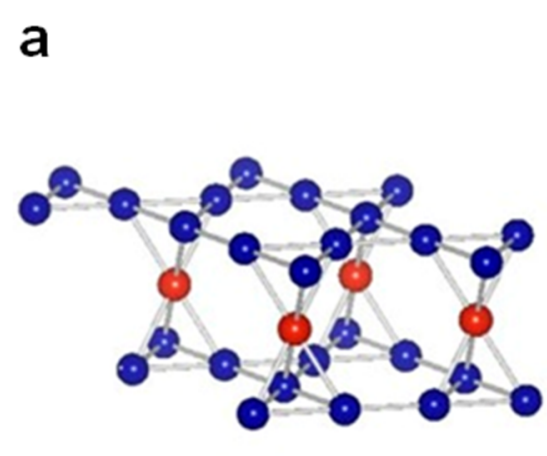

b
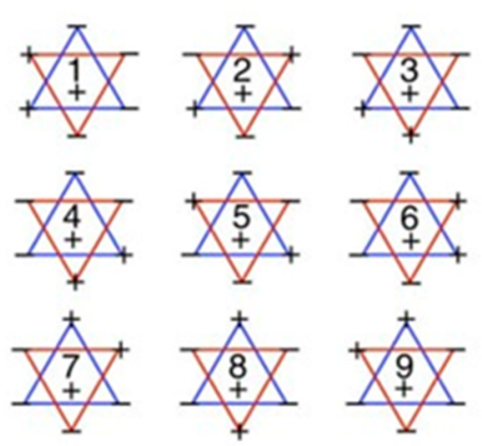

C
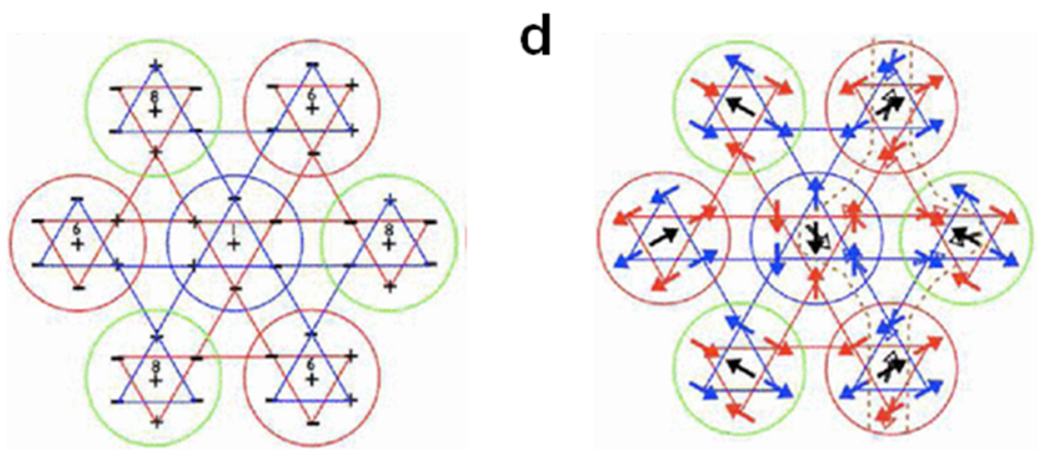

Figure 1.4 The triangular network of bipyramids and its classical ground states

(a) The kagome-triangular-kagome tri-layer, forming the triangular network of bipyramids. Each bipyramid is composed of two corner-sharing tetrahedra. The blue and red spheres represent kagome and triangular sites, respectively. (b) The internally collinear states for each bipyramid are categorized by assigning for each spin a binary sign (representing a parallel $(+)$ or antiparallel (-) direction relative to the color). These states may be viewed as the 18 elements of $Z_{2} \times Z_{3} \times Z_{3}$ by first specifying the sign of the central spin, and specifying one spin in the upper and the lower layers of the bipyramid with the same sign. For visualization, we simply label the first nine states numerically 1..9, and the counterpart (associated with flipping all the signs) as 10..18. (c) A sign state for the triangular lattice of bipyramids that has a long-range $\sqrt{3} \times \sqrt{3}$ structure. (d) Filled arrows represent spins in a collinear bipyramid spin state constructed by the color and the 1-6-8 sign state shown in (c). [5]

Any LC state can be easily obtained by mapping sign states on a long-ranged order triangular lattice with a rule of three spins on a linking triangle must have the same sign, as shown in Figure 1.4 (c). Thus, LC states can be systematically studied using a simple problem of two degrees of freedom: tri-colour representing the three directions of $\sqrt{3} \times \sqrt{3}$ global order and sign states. [5] In a counting exercise, one can always find a finite number of LC states for a given superlattice with nearest neighbour bipyramids. For example, there are only 111 LC states for 6 nearest neighbour bipyramids and 13238 LC states for 12 bipyramids. A LC-state made out of only three sign states holds a long-range order. Since 
one can find only six combinations of sign states which can form long-range orders without violating the equal sign rule of linking triangles, there exist only 36 long-range ordered LC states for any size of the superlattice. [4]

Moreover, the noncollinear and coplanar bipyramid states can be obtained from LC states by collectively rotating each pair of antiparallel spins in each tetrahedron that can be parameterized by three angles. [4] In the mean-field level, these collective rotations between different ground state configurations can be done without any energy cost and LC states are continuously connected by the collective global rotations through their resulting coplanar states, and hence the classical ground state is a flat manifold.

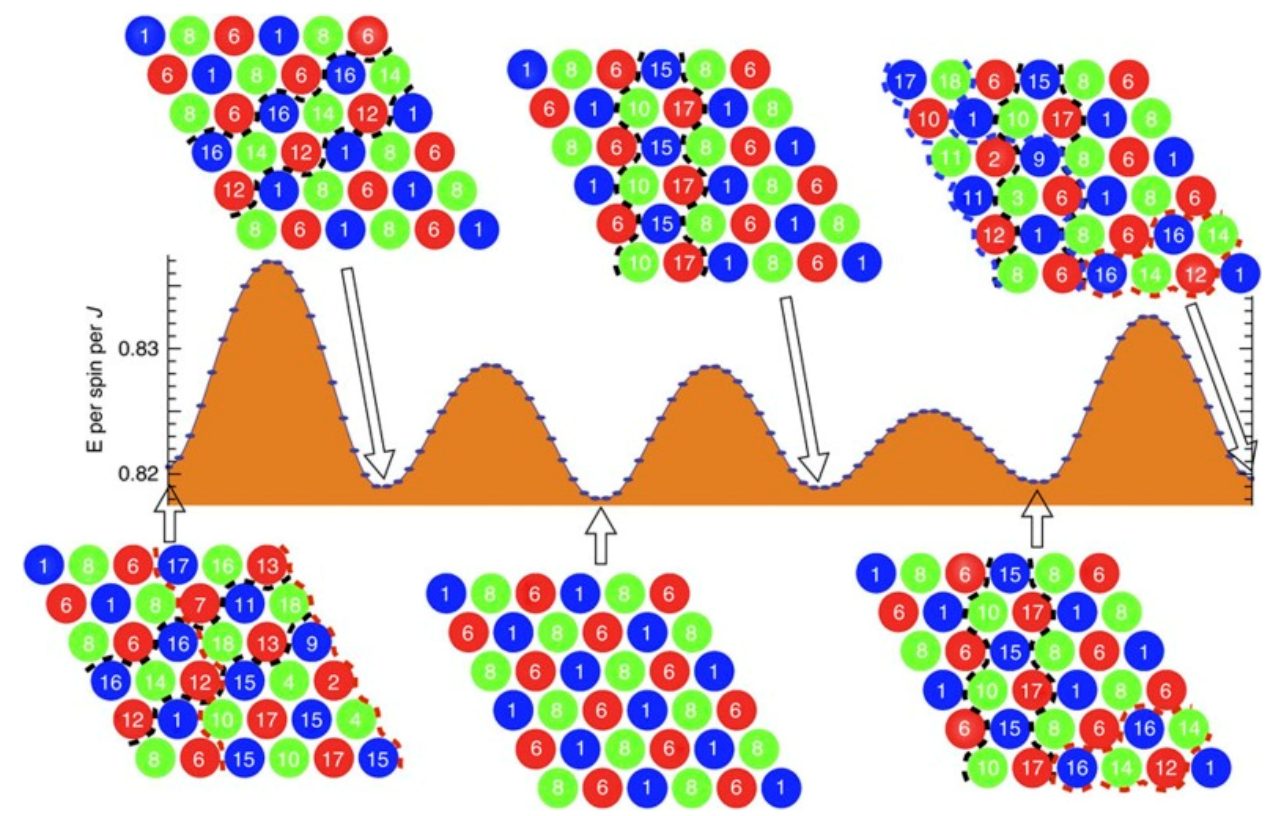

Figure 1.5 Rugged energy landscape induced by quantum fluctuations

The magnetic energy of the quantum fluctuations was calculated for several LC (sign) states near one global minimum. The energy barriers between the minima are composed of the coplanar bipyramid spin states that connect the sign states. [5]

However, the energy cost of quantum fluctuations associated with different ground state configurations are different as estimated within the harmonic (Holstein-Primakoff) approximation and all the LC states have minimum energy compared to the surrounding coplanar state by creating local minima around a LC state in the ground state manifold as shown in Figure 1.5. [5] Moreover, different LC states also have different energy corrections by lifting the classical degeneracy among LC states and long-range ordered LC 
states are the global minimums. Thus, as a result of this quantum fluctuation induced ruggedness in the energy landscape, the system gets trapped in one of the local minima of LC states with or without long-range order, upon cooling. This is an order-by-disorder mechanism for a quasi-2D KTK lattice, and the low-temperature frozen state is termed a spin-jam. [5]

Moreover, the $\mathrm{O}(3)$ invariance of our Heisenberg Hamiltonian is broken explicitly upon spin freezing, and the low-temperature thermodynamics will be governed by low energy hydrodynamic Halperin-Saslow (HS) modes. [15--17] These HS modes are linearly dispersive and lead to a quadratic temperature dependence of heat capacity for quasi-twodimensional systems. Furthermore, the frequency dependence of the imaginary part of the dynamic susceptibility $\left(\chi^{\prime \prime}(\omega)\right)$ shows a linear dependence at low frequencies $(\omega)$ as a consequence of HS modes in low temperature phase. [5]

\subsection{Outline of the Thesis}

In Chapter 02, We give a brief introduction to the experimental and simulation methods used throughout the projects, discussed in this dissertation. Bulk experiments such as direct-current (DC) susceptibility and alternating-current (AC) susceptibility experiments, as well as neutron scattering techniques such as time-of-flight, backscattering and neutron spin echo (NSE) spectroscopy, will be discussed along with the relevant theoretical background. Later in this chapter, Monte-Carlo (MC) simulation techniques such as Metropolis sampling and Langevin dynamics relevant for spin systems will also be discussed.

In Chapter 03, We will introduce two isostructural frustrated magnetic systems, $\mathrm{BaCr}_{9 p} \mathrm{Ga}_{12-9 p} \mathrm{O}_{19}[\mathrm{BCGO}(p)]$ and $\mathrm{SrCr}_{9 p} \mathrm{Ga}_{12-9 p} \mathrm{O}_{19}[\mathrm{SCGO}(p)]$ in which $\mathrm{Cr}^{3+}$ ions arranged in a magnetoplumbite lattice and their low-temperature properties using bulk susceptibility measurements as well as neutron scattering experiments. The main purpose of this chapter is to introduce the low-temperature properties of $\operatorname{SCGO}(p) / \operatorname{BCGO}(p)$ in the clean limit $(p \rightarrow 1)$. Thus, starting from a broad discussion of DC and AC susceptibility in $\operatorname{SCGO}(p) / \operatorname{BCGO}(p)$ as a function of $p$, we will narrow down the discussion to $\operatorname{BCGO}(p=$ 0.902(8)) later in the Chapter. Inelastic neutron scattering experiments as well as Neutron 
Spin Echo experiments are performed on $\operatorname{BCGO}(p=0.902(8))$ sample and the results will be discussed as a function of temperature and energy resolution. The experimental evidence for a quantum fluctuation induced spin jam behavior in the clean limit of $\operatorname{BCGO}(p)$ will be given.

In Chapter 04, $\operatorname{SCGO}(p)$ will be studied as a function of dilution ( $p$ ) using both bulk susceptibility and neutron scattering experiments, and the $p$-T phase diagram for $\operatorname{SCGO}(p)$ will be generated in compliance with the experimental results. Both static and dynamical scattering functions are studies for six powder samples, $\operatorname{SCGO}(p=0.968(6)), \operatorname{SCGO}(p=$ $0.917(9)), \operatorname{SCGO}(p=0.844(8)), \operatorname{SCGO}(p=0.777(6)), \operatorname{SCGO}(p=0.620(6))$ and $\operatorname{SCGO}(p$ $=0.459(5))$ measured at a neutron time-of-flight spectrometer, as a function of wavevector $(Q)$, transfer energy $(\hbar \omega)$ and temperature $(T)$. The hydrodynamical model proposed by Halprin and Saslow will be used to explain the unconventional dynamics observed in the clean limit of $\operatorname{SCGO}(p)$ and experimental evidence to support the spin jam theory will be presented along with semi-classical spinwave calculations.

Chapter 05 will present a comparative study done on three different magnetic glasses using bulk and neutron scattering techniques. The systems studied here, are a Quasi-2D frustrated magnet, $\mathrm{BCGO}(p=0.902(8))$, a three-dimensional (3D) pyrochlore magnet, $\mathrm{Y}_{2} \mathrm{Mo}_{2} \mathrm{O}_{7}$ and an archetypical spin glass, $\mathrm{Cu}-2$ at. $\% \mathrm{Mn}(\mathrm{CuMn} 2 \%)$. The magnetic order parameters of these materials were studied as a function of instrumental resolution $\left(\Delta \omega_{0}\right)$ spanning over ten decades of frequency using AC susceptibility, Neutron Spin Echo spectroscopy, Neutron Backscattering spectroscopy and Neutron time-of-flight spectroscopy. The temperature evolution of relaxation-time distribution is estimated by means of orderparameter measurements for each compound. Furthermore, the frequency dependent freezing temperature $\left(T_{f}\right)$ modeled by Vogal-Fulcher law, will be used to distinguish different magnetic glasses systematically. Finally, zero-field cooled (ZFC) and field cooled (FC) susceptibility will be studied as a function of applied field and the de AlmeidaThouless (AT) line will be calculated for each magnet. Thus, the chapter will be concluded by proposing a scaling of critical field to identify different magnetic glasses.

In Chapter 6, we present a systematic method to study different topologies of energy landscapes using ageing and memory effect experiments. Magnetic memory measured in 
DC susceptibility of two spin jam materials, $\operatorname{SCGO}(p=0.97)$ and $\operatorname{BCGO}(p=0.96)$ will be presented as a function of waiting time and waiting temperature in comparison to an archetypical spin glass $C u \mathrm{Mn} 2 \%$. The data will be quantitively analyzed and compared against our Monte-Carlo (MC) simulation done on two different barrier-tree models. Furthermore, a non-hierarchical energy landscape with a wide nearly flat rough bottom will be proposed for $\operatorname{SCGO}(p=0.97) / \operatorname{BCGO}(p=0.96)$ in contrast to the hierarchical rugged funnel type energy landscape proposed to explain low-temperature relaxational properties of canonical dilute spin glasses.

In Chapter 7, we will present similar memory effect studies on five different compounds, which can be divided into three categories: (1) the high-temperature superconducting materials, cuprates and Fe-chalcogenides, (2) Kitaev-model-related systems $\mathrm{Li}_{2} \mathrm{RhO}_{3}$ and $\mathrm{Na}_{2} \mathrm{Ir}_{1-x} \mathrm{Ti}_{x} \mathrm{O}_{3}$, and (3) a semi-conducting pyrochlore $\mathrm{Y}_{2} \mathrm{Mo}_{2} \mathrm{O}_{7}$. Moreover, similar memory effect studies on six $C u_{1-x} \mathrm{Mn}_{x}(x=0.02,0.15,0.3,0.45,0.75$ and 0.85$)$ samples will be presented in comparison. The magnetic memory as a function of waiting time will be systematically studied using a modified stretched exponential function, and a universal scaling of magnetic glasses will be discussed.

In Chapter 8, we will study the spin- $S$ Kitaev model by a combined approach of Metropolis sampling and Landau-Lifshitz dynamics. Both dynamical structure factor $S(Q, \omega)$ and spin-spin correlation function $S(r, \omega)$ obtained from classical simulation for the pure Kitaev models (ferromagnetic and antiferromagnetic) will be directly compared with the results from the exact solution of $S-1 / 2$ Kitaev quantum models. The quantum-classical correspondence of pure Kitaev models will also be discussed in terms of temperature evolutions of $S(Q, \omega)$ and $S(r, \omega)$. Finally, classical simulations beyond the Kitaev model will be discussed limited only to nearest neighbor Heisenberg interactions and the signatures of proximate Kitaev liquids will be revealed. 


\section{Chapter 2}

\section{Methods and Formulations}

\subsection{Bulk Experiments}

\subsubsection{Susceptibility Experiments}

In DC susceptibility experiments, we measured the equilibrium magnetization $(M)$ of a sample, at a given temperature $(T)$ and applied magnetic field $(H)$ and the corresponding susceptibility $\left(\chi_{D C}\right)$ was calculated as $\chi_{D C}=M / H$. In general operation, magnetometer oscillates a sample with respect to a set of pickup coils and measures voltage induced by the moving magnetized samples and hence the magnetization of the sample can be estimated by Faraday's law. Notice that a sample needs to be magnetized first before measuring its magnetization. Thus, it is not possible to estimate magnetic susceptibility exactly at $H=0$ from this technique and the lowest possible magnetic field is determined by the sensitivity of the magnetometer.

DC Susceptibility $\left(\chi_{D C}\right)$ measurements are commonly used for preliminary characterization of magnetic materials. As shown in Figure 2.1, we can easily characterize simple magnetic phases such as ferromagnets, antiferromagnets or paramagnets using $\chi_{D C}$ measurements. For example, in simple paramagnet in which magnetic moments are completely decoupled, $\chi_{D C}$ shows a monotonically increasing behavior with decreasing temperature while it shows a discontinuity at a finite temperature whenever there is a magnetic transition. (see Figure 2.1 (a)) Moreover, a pure paramagnet follows the Curie's law, $\chi=C / T$, where $C$ is the Curie constant of the magnet. But a simple ferromagnet (FM) or antiferromagnet (AFM) follows the Curie-Weiss law, $\chi \propto 1 /\left(T-\theta_{c w}\right)$, where $\theta_{c w}$ is 
the Curie-Weiss temperature, and it undergoes a magnetic phase transition at a finite temperature ( $T_{c}$ for FM and $T_{N}$ for AFM) similar to the $\left|\theta_{c w}\right|$. (see Figure 2.1) The $\theta_{c w}$ of a magnetic material represents the averaged interaction energy of the system and can be estimated by a linear fitting to the inverse susceptibility versus temperature as shown in the Figure 2.1(b). Moreover, an antiferromagnet can be identified easily by the low temperature cusp in susceptibility in which the maximum occurs at the magnetic transition temperature.
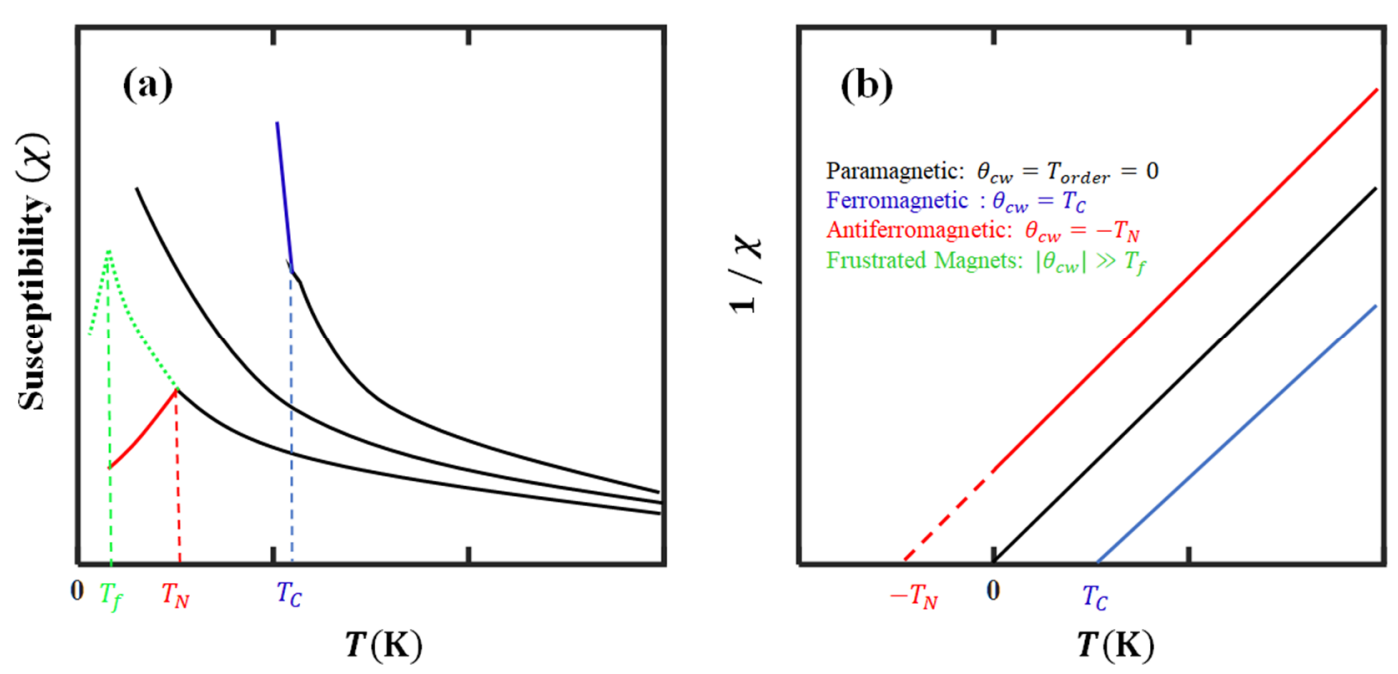

Figure 2.1 Schematic of magnetic susceptibility

Panel (a) shows a schematic of low-temperature susceptibilities in paramagnets, ferromagnets $\left(T_{C}\right)$, antiferromagnets $\left(T_{N}\right)$ and frustrated magnets $\left(T_{f}\right)$. Panel (b) shows a schematic of the inverse susceptibility. By fitting the inverse of magnetic susceptibility in the high-temperature regime, a magnetic material can be characterized preliminarily and the averaged magnetic interaction energy of the system can be estimated.

In frustrated magnets, the phase transition defined by the low-temperature cusp $\left(T_{f}\right)$, will occur at a temperature much lower than the corresponding Curie-Weiss temperature. Thus, a frustrated magnetic system can be easily identified and the degree of frustration of the system can be estimated by the ratio (frustration index) $f=\left|\theta_{c w}\right| / T_{f}$.

Another important experiment of DC magnetization would be zero-field-cooled (ZFC) and field-cooled (FC) measurement. In such experiments, magnetization of a sample is measured as a function temperature followed by continuous cooling to the lowest temperature $\left(T_{\text {base }}\right)$ of the instrument under zero-field or field cooled conditions. When the 
ergodicity of a magnetic phase is broken, ZFC-FC measurements show a bifurcation as function of temperature. This is an intrinsic behavior of magnetic glasses in which energy landscapes are complex and rugged with numerous metastable states. Even though, ZFCFC measurements can be used to detect non-ergodic behaviors of a particular magnetic material, similar experimental features can also be observed in other magnetic systems such as superparamagnets or even in ferromagnets with multiple domains, due to the limited observation time window of the measurement which is about $\sim 100 \mathrm{~s}$. Technically, it is possible to increase the observation time by waiting for a given interval at each temperature step, but the overall experiment might not be feasible due to the limited instrumentation time.
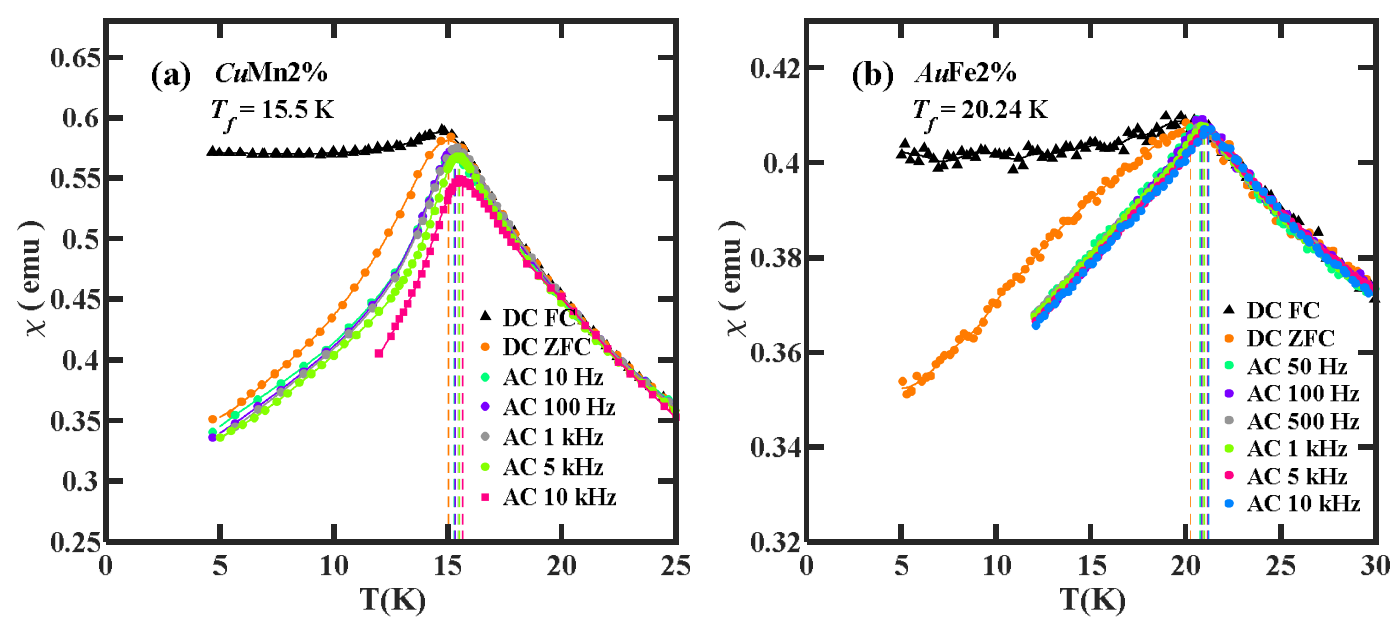

Figure 2.2 Low-T susceptibility measurements of two Spin Glasses: $\mathrm{CuMn2 \%}$ and AuFe2\% The low-temperature magnetic susceptibility measurements of two dilute spin glasses: (a) $\mathrm{CuMn} 2 \%$ and (b) $A u \mathrm{Fe} 2 \%$.

Figure 2.2 shows magnetization measurements of two canonical spin glasses, $\mathrm{Cu}-2$ at. \%

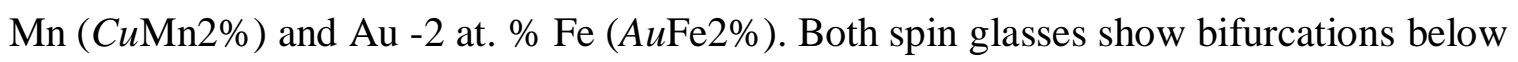
the corresponding cusp of $\chi_{Z F C}$ and it is one of the experimental signatures of spin glasses. The irreversibility temperature $T_{i r r}$ where non-ergodicity begins to expose, is defined as the bifurcation of ZFC-FC curves. Usually, $T_{i r r} / T_{D C} \approx 1$ in magnetic glasses and the ratio decreases with increasing applied field. When the applied field is high enough to modify the complex energy landscape into a smooth pointy vase like landscape with single minimum, ZFC-FC bifurcation will disappear. But, some unconventional glasses might 
show a ZFC-FC splitting at higher temperatures than $T_{D C}$ and it is usually the case for superparamagnets. [9]

\subsubsection{AC Susceptibility Experiments}

In AC susceptibility experiments, the time-dependent magnetization of sample is measured by applying a small time-varying magnetic field. In general operation of ACmagnetometers, the voltage of the pick-up coil is measured without moving the sample and, the amplitude $(A)$ and the phase shift $(\phi)$ of the time varying magnetization is estimated by comparing the output voltage signal with the driver signal. Thus, the time-

varying susceptibility $\chi(\omega)=\frac{M(\omega)}{H_{A C}}=\frac{M_{A C}}{H_{A C}} \mathrm{e}^{i \phi}$ where $M_{A C}$ and $H_{A C}$ are the amplitudes of magnetization signal and the driver field respectively, is calculated and the real and imaginary parts of the susceptibilities ( $\chi^{\prime}$ and $\left.\chi^{\prime \prime}\right)$ are estimated as:

$$
\begin{gathered}
\chi(\omega)=\chi^{\prime}(\omega)+i \chi^{\prime \prime}(\omega) ; \quad \chi_{A C}=M_{A C} / H_{A C} \\
\chi^{\prime}(\omega)=\chi_{A C} \cos (\phi) \\
\chi^{\prime \prime}(\omega)=\chi_{A C} \sin (\phi)
\end{gathered}
$$

The amplitude of susceptibility $\chi_{A C}$ measures the slope of the $M(H)$ curve, $d M / d H . \chi^{\prime}$ and $\chi^{\prime \prime}$ measure the dynamical susceptibility and absorption nature of the magnetic system respectively. [18]

Both $\chi^{\prime}$ and $\chi^{\prime \prime}$ are sensitive to magnetic phase transitions and show sharp changes at the critical temperature. Since different magnetic phases show different characteristic signatures on AC susceptibilities as a function of temperature, the current discussion will focus on magnetic glasses only. Figure 2.3 shows $\chi^{\prime}$ and $\chi^{\prime \prime}$, as a function of temperature, for a 3D Pyrochlore magnet, $\mathrm{LuYMo}_{1.4} \mathrm{Ti}_{0.6} \mathrm{O}_{7}$, whose parent compound $\mathrm{Y}_{2} \mathrm{Mo}_{2} \mathrm{O}_{7}$ is a well-known magnetic glass. [19,20] At low temperature, $\chi^{\prime}$ shows a cusp similar to the ZFC susceptibility measurements and $\chi^{\prime \prime}$ also shows an acute change at around the same temperature, implying a magnetic phase transition at $T \sim 6 \mathrm{~K}$. Moreover, $\chi^{\prime \prime}$ shows another change at around $100 \mathrm{~K}$ where high temperature susceptibility deviates from Curie-Weiss law. The deviation from Curie-Weiss law usually happens upon development of spin-spin correlation in a magnetic system and $\chi^{\prime \prime}$ is sensitive to this. Hence $\chi^{\prime \prime}$ can be used to detect 
magnetic crossovers such as paramagnetic (spin gas) to co-operative paramagnetic (spin liquid) crossover just like in the current example.

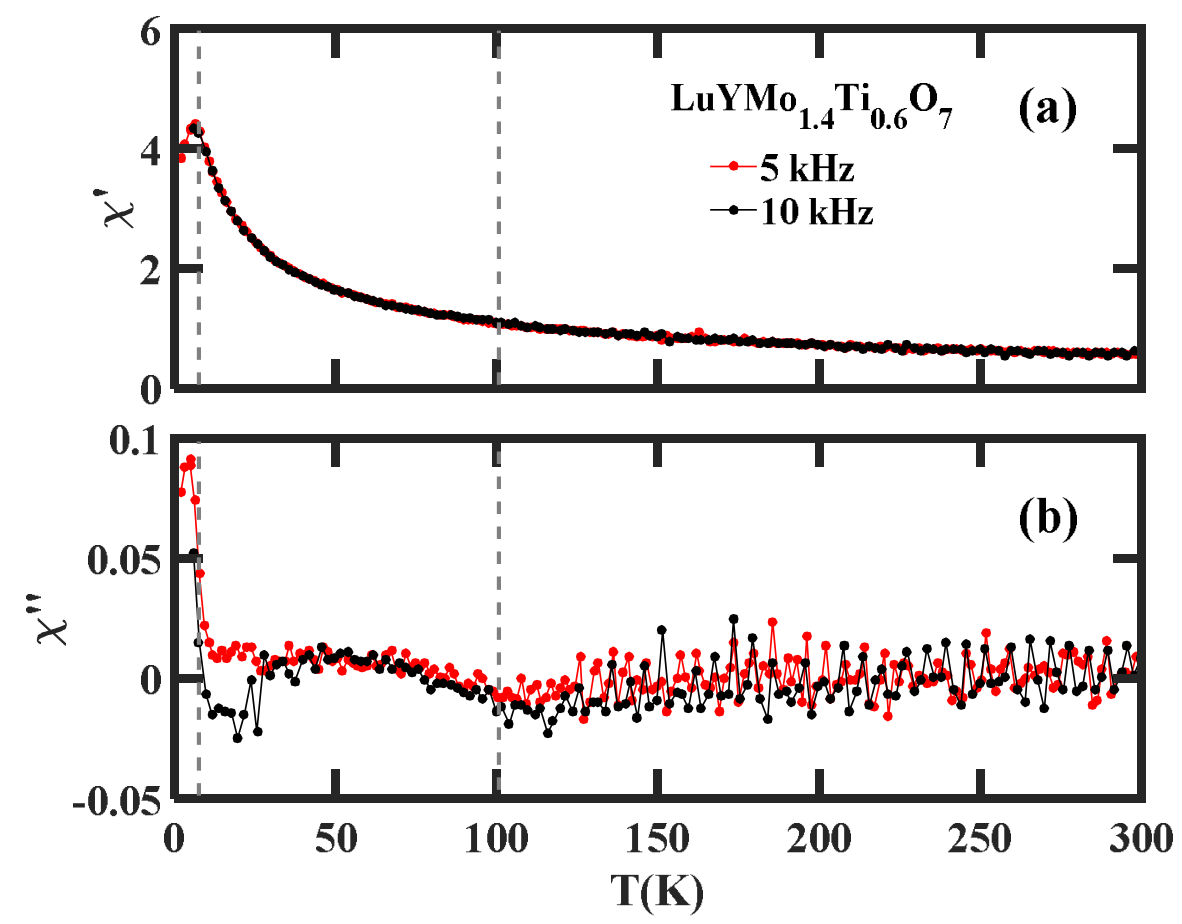

Figure 2.3 High-Temperature AC susceptibility of $\mathrm{LuYMo}_{1.4} \mathrm{Ti}_{0.6} \mathrm{O}_{7}$

The high temperature AC magnetization of a 3D pyrochlore magnet, $\mathrm{LuYMo}_{1.4} \mathrm{Ti}_{0.6} \mathrm{O}_{7}$ which shows magnetic glass behavior at low temperature regime, is measured for a wide temperature range and the development of dynamical spin correlation with decreasing temperature. The panels (a) and (b) summarize the real and imaginary parts of the AC magnetization respectively.

As shown in Figure 2.2, the $\chi^{\prime}$ shows a cusp at low temperature and the cusp temperature $\left(T_{f}\right)$ shifts to higher temperatures with increasing frequency. This is a typical signature for magnetic systems with multiple relaxational dynamics such as magnetic glasses or superparamagnets. Different magnetic systems have different relaxational behaviors and the frequency dependence of $T_{f}$ can be used to identify them. For example, spin glasses usually follow Vogal-Fulcher law, $f=f_{0} \exp \left[-E_{a} / k_{B}\left(T_{f}-T_{0}\right)\right]$, while superparamagnets shows just a thermally activated behavior (Arrhenius law, $f=$ $\left.f_{0} \exp \left[-E_{a} / k_{B} T_{f}\right]\right)$. However, the accessible frequency range of AC magnetometry is 
limited as $0.01 \mathrm{~Hz}$ to $10 \mathrm{kHz}$. To access faster spin relaxational dynamics, we need to consider other techniques as Neutron Spectroscopy or Muon spin spectroscopy.

\subsection{Neutron Scattering Experiments}

\subsubsection{Background}

Neutron scattering is a powerful experimental technique used in modern scientific research of crystallography, physics, physical chemistry, biology and material science. Unlike other experimental techniques, scattering experiments can reveal both spatial and temporal information of matter simultaneously. The microscopic techniques such as Muon Spin Resonance ( $\mu \mathrm{SR})$, Nuclear Magnetic Resonance (NMR) or Mössbauer spectroscopy, probe local behaviour at spin-spin correlation length, $R=0$ (that is, the averaged behavior over all the wavevectors $Q$ ), while the bulk techniques such as AC-susceptibility or Electron Spin Resonance (ESR), measure averaged behavior over the macroscopic volumes of a sample $(Q=0)$. Among many scattering methods, neutron scattering is special since neutrons can penetrate deeper into the condensed matter systems as a consequence of its zero net-charge. Neutrons can be used to probe atomic nuclei as well as microscopic magnetic field distributions, usually caused by unpaired electrons of magnetic ions in a sample, without interacting with the electron cloud of the material, unlike other scattering particles such as electrons or X-rays (photon).

There are two fundamental mechanisms of neutron production for scattering purposes: nuclear fission and spallation of heavy nuclei. In nuclear fission, a neutron is absorbed by a fissile nucleus such as ${ }^{235} \mathrm{U}$ or ${ }^{239} \mathrm{Pu}$, which then splits into smaller nuclei by producing free neutrons and gamma radiation. A single fission reaction produces 2.5 neutrons on average. In accelerator driven spallation sources, a high-energy proton beam is focused to a dense heavy metal target made out of high-mass-number materials such as uranium, tungsten, tantalum or mercury, and neutrons are produced from the collisions along with other subatomic particles such as pion or kaon. Practically, about 20 neutrons can be produced from a single proton at spallation sources. To compare two neutron sources, the neutron flux from a reactor source is continuous while it is pulsed from a spallation source. 
Moreover, the energy distribution of a neutron flux is either Maxwellian for a reactor source or a "slowing-down" spectrum for spallation source.

Neutrons are usually named based on their energy/wavelength. There are many energy ranges are defined for Neutrons [21]: Cold, Thermal, and Hot neutrons are typically found in neutron research facilities, and only first two are useful for life-science research. Usually, neutron moderators are used to reduce the speed of neutrons to the required range of energy. Different mediums can be used as neutron moderators depending on the required energy range as summarized in Table 2-1. There are different kinds of neutron scattering instruments, which use either cold or thermal neutrons, available in neutron research facilities; but only the neutron scattering techniques relevant to this thesis will be discussed in the rest of this section.

Table 2-1 Comparison of Cold, Thermal and Hot neutrons.

\begin{tabular}{lccc}
\hline \hline & Cold & Thermal & Hot \\
\hline Neutron wavelength & $3-20 \AA$ & $1-3 \AA$ & $0.3-1 \AA$ \\
Sample length scale & $1 \AA-100 \mathrm{~nm}$ & $0.3-5 \AA$ & $0.1-2 \AA$ \\
Sample time scale & $1 \mathrm{kHz}-1 \mathrm{THz}$ & $0.1-10 \mathrm{THz}$ & $1-100 \mathrm{THz}$ \\
Moderator & Liquid $\mathrm{D}_{2}$ & Liquid $\mathrm{D}_{2} \mathrm{O}$ & graphite \\
Moderator & $20 \mathrm{~K}$ & $300 \mathrm{~K}$ & $2000 \mathrm{~K}$ \\
Temperature & & & \\
\hline
\end{tabular}

\subsubsection{Theory of Scattering Experiments}

In the typical operations of a neutron scattering experiment, a beam of neutrons is scattered by a sample, and the scattered neutrons are counted as a function of solid angle and energy using neutron detectors. (see Figure 2.4 (a)) The momentum transfer wavevector $\vec{Q}$ and the energy transfer $\hbar \omega$ of the scattering process are formulated as, 


$$
\begin{aligned}
\vec{Q} & =\vec{k}_{i}-\vec{k}_{f} \\
\hbar \omega & =E_{i}-E_{f}
\end{aligned}
$$

where $\vec{k}_{i}$ and $\vec{k}_{f}$ are the incident and the scattered wavevectors, $E_{i}$ and $E_{f}$ are the incident and final energy of neutrons respectively. The relation of the energy and momentum can be written as:

$$
E=\frac{\hbar^{2} k^{2}}{2 m_{n}}
$$

where, $m_{n}$ is the mass of neutron.

The experiments that follow the elastic scattering relation of $\left|\vec{k}_{i}\right|=\left|\vec{k}_{f}\right|=k$ are usually called either elastic neutron scattering or neutron diffraction experiments, and the wavevector $(Q=|\vec{Q}|)$ for a given scattering angle $(\theta)$ can be easily calculated by the Bragg's Law,

$$
Q=\frac{4 \pi}{\lambda} \sin \left(\frac{\theta}{2}\right)
$$
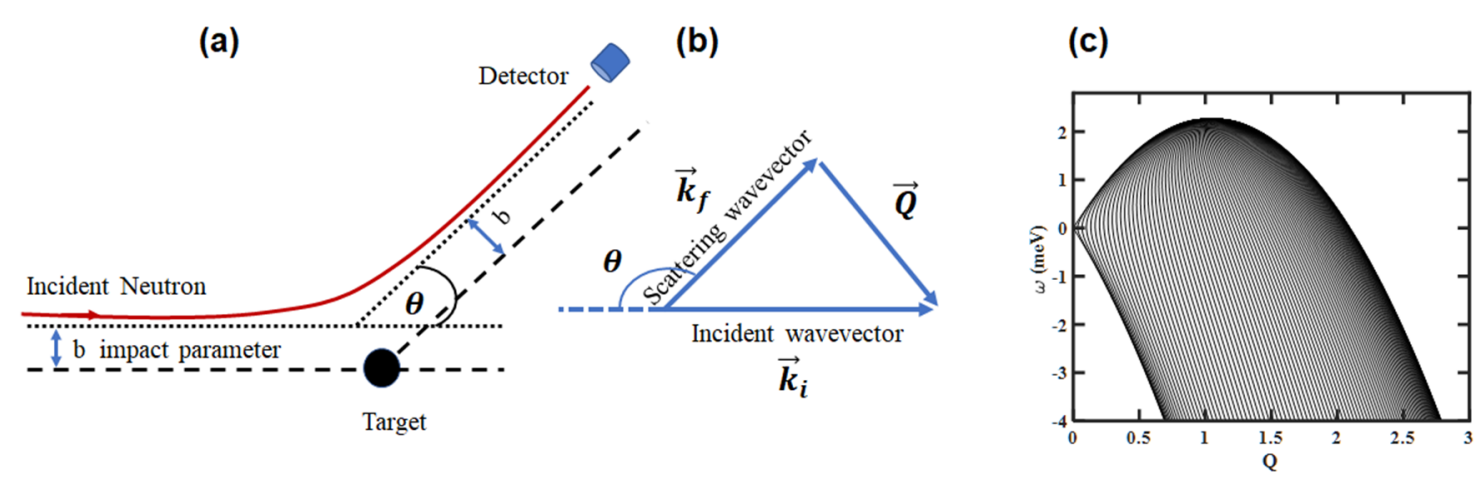

Figure 2.4 Schematics of neutron scattering theory

(a) The schematic of a neutron scattering experiment. (b) The scattering triangle of the experiment where $\vec{k}_{i}$ and $\vec{k}_{f}$ are the incident and the scattered wavevectors respectively. (c) The coverage of energy-momentum space for a neutron scattering experiment with an incident beam of wavelength $\lambda=6 \AA$.

In neutron spectroscopy, the energy transfer $(\hbar \omega)$ of a scattered neutron will be measured as well as momentum transfer $(Q)$, and the accessible energy-momentum space is 
determined by Equation 2.5. A sketch of energy-momentum space for a neutron scattering experiment with an incident beam of wavelength $\lambda=6 \AA$, is shown in the Figure 2.4(c).

$$
\begin{gathered}
Q=\sqrt{\left|\vec{k}_{i}\right|^{2}+\left|\vec{k}_{f}\right|^{2}-2\left|\vec{k}_{i}\right|\left|\vec{k}_{f}\right| \cos (2 \theta)} \\
Q^{2}=\frac{2 m_{n}}{\hbar^{2}}\left(2 E_{i}-\hbar \omega-2 \sqrt{E_{i}\left(E_{i}-\hbar \omega\right)}\right) \cos (\theta) \\
\left(\frac{\hbar^{2}}{2 m_{n}} \sim 2.072 \mathrm{eV}^{-1} \cdot \operatorname{rad}^{-2} \cdot \mathrm{m}^{-2}\right)
\end{gathered}
$$

The neutron scattering spectrometers are utilized with two different scattering geometries, direct-geometry, and indirect-geometry. In the case of direct-geometry setting, the incident energy of the experiment $\left(E_{i}\right)$ is fixed by means of either neutron monochromators or choppers. In the case of indirect-geometry, the energy of scattered neutrons $\left(E_{f}\right)$ are fixed by the analyzer crystals and, this technique is usually called Neutron Backscattering Spectroscopy. Among many different spectrometers, Neutron time-of-flight (TOF) spectrometers are quite common in inelastic neutron scattering spectroscopy and usually operates in direct-geometry setting. In a standard TOF spectrometer, the incident beam is pulsed such that the initial position and the velocity of each pulse is known, and the neutrons will be counted as a function of position and flight-time of scattered neutrons. Thus, the scattering cross-section of the experiment as a function of $\hbar \omega$ and $Q$, can be constructed from the flight-time $\left(t_{f}\right)$ and scattering angle $(\theta)$ information using Equation 2.6.

$$
\begin{gathered}
\hbar \omega=\frac{1}{2} m_{n}\left(v_{i}^{2}-v_{f}^{2}\right) \\
v_{i}=\frac{h}{m_{n} \lambda_{0}} \approx \frac{3.956}{\lambda_{0}} ; \quad v_{f}=\frac{d_{2}}{t_{f}}
\end{gathered}
$$

where $\lambda_{0}$ is the incident wavelength of the beam and $d_{2}$ is the sample to detector distance. In TOF spectrometers at reactor facilities, the continuous neutron flux is turned into a 
monochromatic pulsed beam by a set of choppers (usually six to seven choppers). A schematic of a reactor based TOF instrument is shown in Figure 2.5. [22]

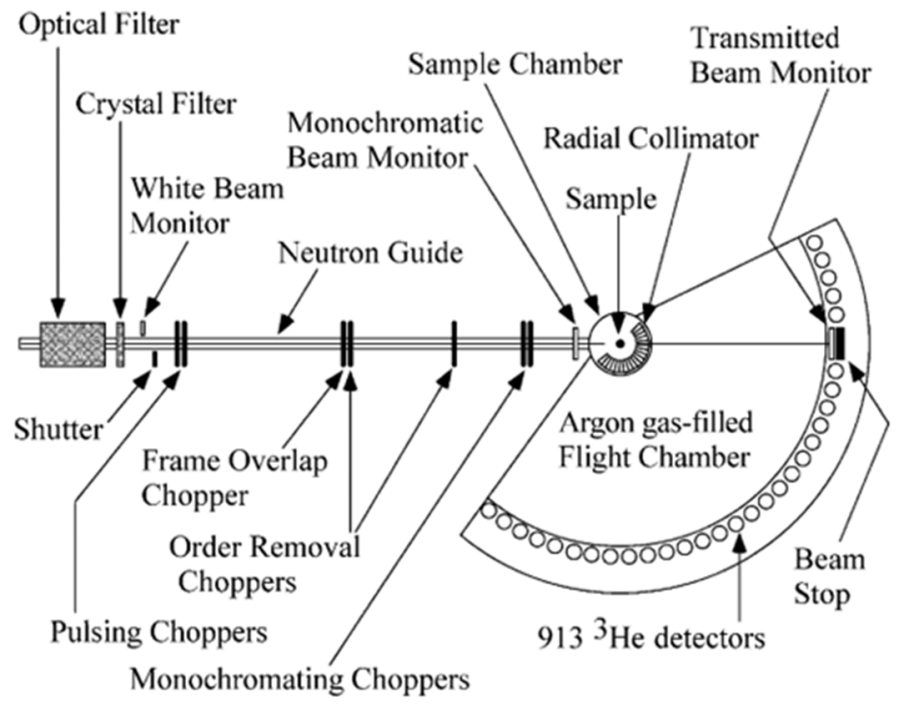

Figure 2.5 Schematics of a Neutron Time-of-Flight spectrometer

This is a detailed schematic of the Disk Chopper Spectrometer (DCS) at National Institute of Standards and Technology (NIST). [22]

In most of the inelastic neutron scattering experiments, we measure the partial differential scattering cross-section as defined below:

$\frac{d^{2} \sigma}{d \Omega d E_{f}}=\frac{\text { Number of scattered neutrons per second into } d \Omega \text { with energies }\left[E_{f}, E_{f}+d E_{f}\right]}{\Phi d \Omega d E_{f}}$

Here, $E_{f}$ is the final energy of the neutron and $\Phi$ is the flux of the neutron beam, defined as the number of incident neutrons per unit-area perpendicular to the neutron beam per second. [23] The differential scattering cross-section for an inelastic scattering process in which the initial and final states of the sample are denoted as $\left|\lambda_{i}\right\rangle$ and $\left|\lambda_{f}\right\rangle$ respectively, can be written as the following relation by mean of Fermi's Golden Rule, 


$$
\frac{d^{2} \sigma}{d \Omega d \omega}=\frac{k_{f}}{k_{i}}\left(\frac{m_{n}}{2 \pi \hbar^{2}}\right)^{2}\left|\left\langle\overrightarrow{k_{l}} \lambda_{i}|\hat{V}| \overrightarrow{k_{f}} \lambda_{f}\right\rangle\right|^{2} \delta\left(\hbar \omega+E_{i}-E_{f}\right)
$$

where, $\hat{V}$ is the interaction potential of the sample. With the assumption of weak interaction between neutrons and the sample, the incoming and outgoing neutrons can be treated as plane waves and the matrix element in Equation 2.8 can be written as,

$$
\left\langle\overrightarrow{k_{l}} \lambda_{i}|\hat{V}| \overrightarrow{k_{f}} \lambda_{f}\right\rangle=\left(\frac{2 \pi \hbar^{2}}{m_{n}}\right)\left\langle\lambda_{i}\left|\sum_{l} b_{l} \cdot e^{i \vec{Q} \cdot \overrightarrow{r_{l}}}\right| \overrightarrow{k_{f}} \lambda_{f}\right\rangle
$$

where $r_{l}$ and $b_{l}$ are the coordinates and the nuclear scattering length of the $l^{\text {th }}$ scattering center. Assuming that there are no correlations between scattering length of different nuclei, we can derive the scattering cross-section for a monoatomic system using Equation 2.8 and 2.9 as shown below.

$$
\begin{gathered}
\frac{d^{2} \sigma}{d \Omega d \omega}=\frac{k_{f}}{k_{i}}\left(\frac{m_{n}}{2 \pi \hbar^{2}}\right)^{2}\left[\frac{\sigma_{c o h}}{4 \pi} \sum_{i j} \int_{-\infty}^{\infty}\left\langle e^{-i \vec{Q} \cdot R_{i}(0)} e^{-i \vec{Q} \cdot R_{j}(t)}\right\rangle e^{-i \omega t} d t\right. \\
\left.+\frac{\sigma_{i n c}}{4 \pi} \sum_{i} \int_{-\infty}^{\infty}\left\langle e^{-i \vec{Q} \cdot R_{i}(0)} e^{-i \vec{Q} \cdot R_{i}(t)}\right\rangle e^{-i \omega t} d t\right] \\
\sigma_{c o h}=4 \pi(\bar{b})^{2} \quad \sigma_{i n c}=4 \pi\left(\overline{b^{2}}-(\bar{b})^{2}\right) \quad r_{l}=N+R_{i}
\end{gathered}
$$

where $\bar{b}$ is the average scattering length of the system. The first term of Equation 2.10 is the coherent nuclear scattering contribution from the correlation between different atoms, while the second term is the incoherent nuclear scattering contribution from selfcorrelations of atoms.

In a magnetic system in which the unpaired electrons in the outer shells of ions introduce an effective magnetic moment at each ionic coordinates, the neutrons can be mainly scattered by the angular momentum of the nucleus $(I)$, the spin angular momentum $(S)$ and orbital angular momentum $(L)$ of unpaired electrons. For ions with even atomic and mass 
numbers, the nuclear angular momentum $I$ is technically zero since the equal number of neutrons and protons cancels the net moment of the nucleus. Moreover, the nuclear contribution from bigger uneven atoms could be negligibly small comparing to the contributions from $S$ and $L$ components of the system. The inelastic neutron magnetic scattering cross-section for unpolarized neutrons can be written as: [23,24]

$$
\begin{gathered}
\frac{d^{2} \sigma}{d \Omega d \omega}=\frac{k_{f}}{k_{i}} \frac{r_{0}^{2}}{2 \pi \hbar}\left\{\frac{1}{2} g F(Q)\right\}^{2} \cdot \exp (-2 W(Q)) \times \sum_{\alpha, \beta}\left(\delta_{\alpha \beta}-\hat{Q}_{\alpha} \hat{Q}_{\beta}\right) S^{\alpha \beta}(Q, \omega) \\
S^{\alpha \beta}(Q, \omega)=\int_{-\infty}^{\infty}\left\langle\hat{S}_{Q}^{\alpha}(0) \hat{S}_{-Q}^{\beta}(t)\right\rangle e^{-i \omega t} d t
\end{gathered}
$$

where, $r_{0}=\gamma e^{2} / m_{e} c^{2}$ and $\gamma$ is the gyromagnetic ratio $(\gamma \approx 1.913$ for neutrons) and $g$ is the Landé splitting factor,

$$
\begin{gathered}
g=g_{S}+g_{L} \\
g_{S}=1+\frac{S(S+1)-L(L+1)}{J(J+1)} \\
g_{L}=\frac{1}{2}+\frac{L(L+1)-S(S+1)}{2 J(J+1)}
\end{gathered}
$$

$F(Q)$ is the magnetic form factor and it can be written as Equation 2.13 within the dipole approximation. [25]

$$
\begin{gathered}
F(Q)=\left\langle j_{0}(Q)\right\rangle+\left(1-\frac{2}{g}\right)\left\langle j_{2}(Q)\right\rangle \\
\left\langle j_{0}(Q)\right\rangle=A \exp \left(-a s^{2}\right)+B \exp \left(-b s^{2}\right)+C \exp \left(-c s^{2}\right)+D \\
\left\langle j_{l}(Q)\right\rangle=A \mathrm{~s}^{2} \exp \left(-a s^{2}\right)+B \mathrm{~s}^{2} \exp \left(-b s^{2}\right)+C \mathrm{~s}^{2} \exp \left(-c s^{2}\right)+D \mathrm{~s}^{2} ; \quad l=2,4,6, \ldots
\end{gathered}
$$

where $s=\sin (\theta) / \lambda=Q / 4 \pi$ and the coefficients $A, B, C, D, a, b, c$, and $d$, can be found in the Ref. [25]. The term, $\exp (-2 W(Q))$ is the Debye-Waller factor which describes the thermal fluctuations of the lattice and it can be approximated as unity for low temperatures where most of the magnetic transitions happen. The $S^{\alpha \beta}(Q, \omega)$ is the magnetic structure factor of the thermally equilibrated magnetic phase of the compound and can also be seen as the Fourier transformation of the real space and time correlation functions. 


\subsubsection{Neutron Backscattering Spectroscopy}

As mentioned in the previous section, the neutron backscattering spectrometers are inversegeometry spectrometers in which the final energy of neutrons $E_{f}$ is fixed by analyzer crystals. In these spectrometers, the analyzer crystals are chosen to have the Bragg reflection angle at or near $90^{\circ}$ (this is the reason why it is called backscattering). $\mathrm{Si}, \mathrm{CaF}_{2}$, GaAs and Graphite are typically used in backscattering monochromators. Thus, the energy resolution of the instrument $(\Delta E)$ depends mostly on the analyzer crystals and can be determined by the following expression: [26]

$$
\Delta E=2 E_{f}\left(\frac{\delta d}{d}+\cot (\theta) \delta \theta\right)
$$

where $\delta d / d$ is the spread of the analyzer lattice constant, and $\theta$ is the Bragg angle. Notice that the energy resolution is minimized when $\theta$ approaches $90^{\circ}$. Figure 2.6 shows a schematic of the High Flux Backscattering Spectrometer (HFBS) at NIST.

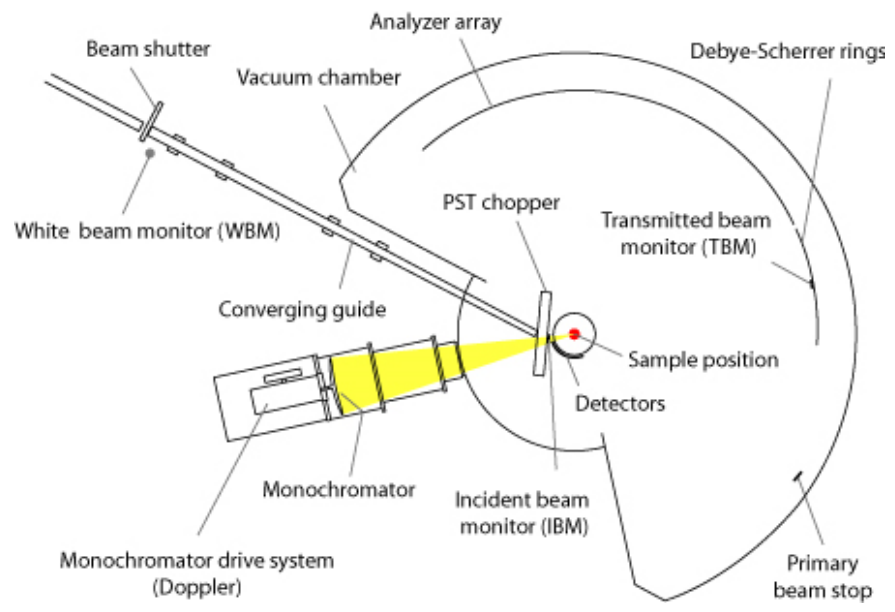

Figure 2.6 A schematic of a neutron backscattering spectrometer

The schematic of the High Flux Backscattering Spectrometer (HFBS) at NIST. [27]

Typically, the analyzed crystals with $\delta d / d \approx 1.86 \times 10^{-5}$ yields $\mu \mathrm{eV}$ energy resolution. Hence the nanosecond scale relaxations can be easily probed with these instruments, unlike the direct-geometry TOF spectrometer which can probe only relaxation faster than nanosecond time scale in standard operation. Since the $E_{f}$ is fixed, a variation of the incident neutron energy $E_{i}$ is a requirement in these spectrometers. Thus, a kind of 
monochromator is required in the reactor based spectrometers to get a pulsed beam from the continuous flux of the reactor source, similar to the reactor based TOF spectrometers. The energy distribution of incident energy is usually achieved either by means of a Doppler-moving monochromator [28] or by varying the temperature of a standing monochromator with respect to the analyzer crystals. [29] An example of former is the HFBS at NIST as shown in Figure 2.6. [27]

The backscattering spectrometers at spallation neutron sources, do not use monochromators. Instead, the neutron's $E_{i}$ is determined from the overall time-of-flight (TOF) between the neutron source and the detectors and hence those spectrometers are called time-of-flight backscattering spectrometers (TOF-BSS). The energy resolution of such spectrometer is determined by the following expression:

$$
\Delta E=2 E_{f} \sqrt{\left(\frac{\delta d}{d}+\cot (\theta) \delta \theta\right)^{2}+\left(\frac{\delta \mathrm{TOF}}{\mathrm{TOF}}\right)^{2}}
$$

where $\delta \mathrm{TOF}$ is the uncertainty of the time-of-flight. The BASIS spectrometer at Spallation Neutron Source (SNS), ORNL is an example of TOF-BSS. [26]

\subsubsection{Neutron Spin Echo Spectroscopy}

Neutron Spin Echo (NSE) spectroscopy is a unique neutron scattering technique, invented by Mezei in 1972 [30]. In this method, the spin polarization of neutrons is treated as the individual clocks to track the flight of each neutron, yielding higher temporal resolution in a neutron scattering experiment. Unlike other neutron scattering techniques, NSE spectroscopy measures the intermediate scattering function $I(Q, t)$, which is the Fourier transformation of typical scattering function $S(Q, \omega)$ of the sample. Generally, NSE spectroscopy allows us to access the time window spanning roughly from $10^{-3}$ to $10^{2} \mathrm{~ns}$. This time-window typically covers from the microscopic timescale of atomic collisions and spin exchange to macroscopic times of slow relaxation processes. 


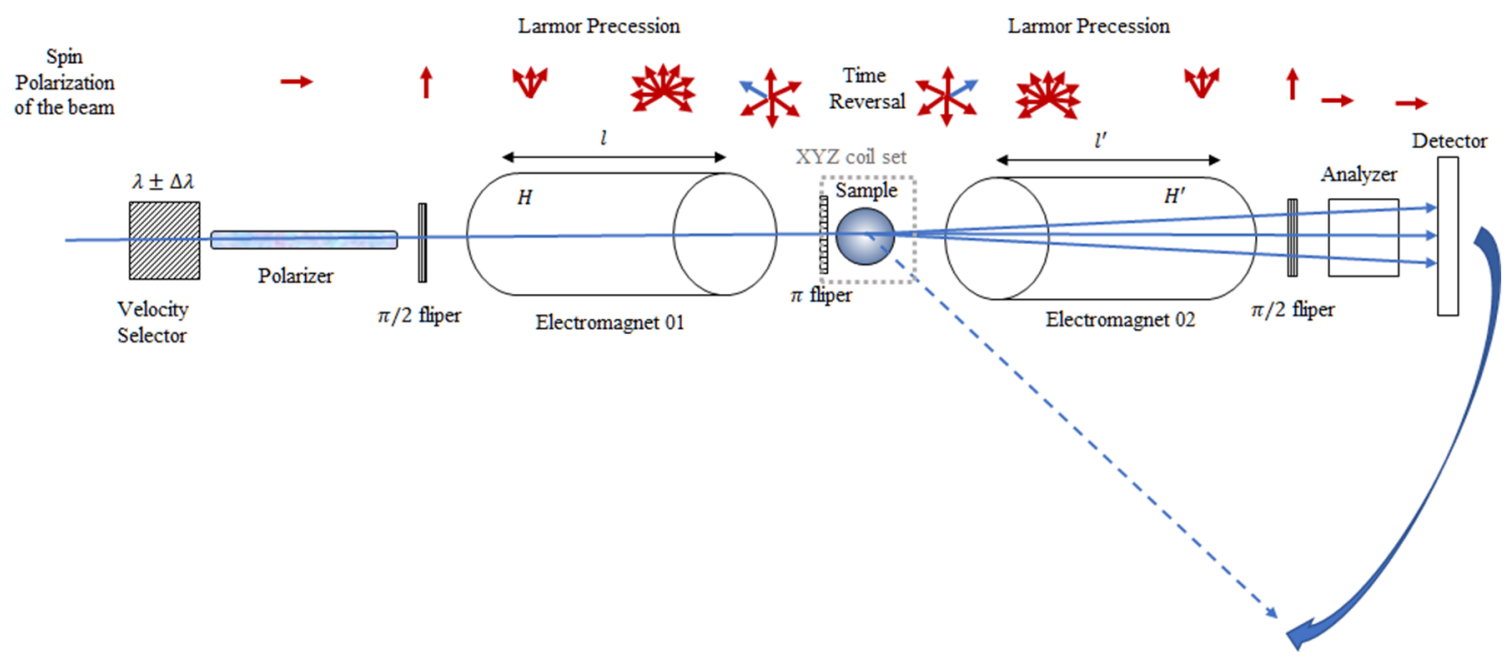

Figure 2.7 Generic setup of a reactor-based neutron spin echo spectrometer

In a standard reactor based neutron spin echo spectrometer (NSE), an unpolarized neutron beam from a reactor source is first sent through a polarizer followed by a velocity selector to obtain a forward longitudinally polarized beam with a wavelength distribution of $\Delta \lambda / \lambda \sim 20 \%$. Then, the longitudinally polarized beam is flipped $90^{\circ}$ by a $\pi / 2$-flipper and send the neutron beam though a coil-solenoid (electromagnet). When there is an effective magnetic field inside the solenoid, the electrons will undergo Larmor precession and the precession angle is a function of magnetic field and the travelling distance. At the end of the solenoid, the beam will hit the sample followed by a $\pi$-flipper which causes an effective "time reversal" to the incoming beam. Then the scattered beam will be sent through another coil-solenoid ideal to the first coil to counter-rotate the spin polarizations of neutrons. At the end, the transverse polarization will be changed to longitudinal by means of a second $\pi / 2$-flipper and analyzed. Thus, the neutron spin echo is measured by varying the magnetic field inside the second electromagnet. This is the typical nuclear scattering setup, but in magnetic NSE spectroscopy, the $\pi$-flipper near sample space is removed and the magnetic sample itself is used for time-reversal operation.

Figure 2.7 illustrates the generic operations of a reactor-based NSE spectrometer. The scattering process begins with a coarse monochromatization $(\Delta \lambda / \lambda \sim 10-20 \%)$ of the incoming continuous unpolarized neutron beam from a reactor source, by means of a velocity-selector. Then, the unpolarized beam will be longitudinally polarized and subsequently it will be turned by $90^{\circ}$ to transverse direction using a $\pi / 2$-flipper. Thus, the transversely polarized beam will enter a neutron guide with uniform magnetic field along the beam direction which is usually a coil solenoid. Then, the neutrons will start to precess perpendicularly to the beam as they travel due to the exerted torque on neutrons by the magnetic field. This is usually called Larmor Precession and the precession angle $(\phi)$ is a function of magnetic field $(H)$ and the traveled time $\left(t_{n}\right)$ : 


$$
\phi=\gamma H t_{n}=\gamma H l / v
$$

where $\gamma=2.916 \mathrm{kHz} /$ Oe is the Larmor constant, $l$ and $v$ are the travelled distance and the velocity of a neutron. Then the plane of precession is inverted by the $\pi$-flipper at the end of the first solenoid and, hence a time-reversal operation is applied to the neutron beam. The $\pi$-flipper is typically used for nuclear scattering experiments but it is ignored during a magnetic scattering experiment, since the effective moments of magnetic ions, themselves work as $\pi$-flippers. Thus, the neutron beam will be scattered by the sample and the scattered beam will be directed to a second solenoid (length $l^{\prime}$ ). Then, the polarization of the scattered neutron will be rotated again by the magnetic field $\left(H^{\prime}\right)$ inside the second solenoid. Thus, total rotation $\left(\phi_{t o t}\right)$ of the process at the end of the second solenoid can be written as:

$$
\phi_{t o t}=-\phi+\phi^{\prime}=-\frac{\gamma H l}{v}+\frac{\gamma H^{\prime} l^{\prime}}{v^{\prime}}
$$

where $\phi^{\prime}$ is the precession angle inside the second solenoid and the minus sign of the first term is due to the time-reversal operation. Thus, the momentum transfer $(\hbar Q)$ and the energy transfer $(\hbar \omega)$ of a scattering event can be written as:

$$
\begin{gathered}
\hbar Q=m v^{\prime}-m v \\
\hbar \omega=\frac{1}{2} m v^{2}-\frac{1}{2} m v^{\prime 2} \cong m v\left(v-v^{\prime}\right)
\end{gathered}
$$

When $H l=H l^{\prime}$, Equation 2.15 can be simplified as,

$$
\phi_{\text {tot }}=\gamma H l\left(\frac{1}{v^{\prime}}-\frac{1}{v}\right) \approx \frac{\gamma H l}{v^{2}}\left(v-v^{\prime}\right) \approx \frac{\gamma H l}{m v^{3}} \hbar \omega
$$

Subsequently, the polarization will be rotated again by an angle of $-90^{\circ}$ using another $\pi / 2$ flipper at the end similar to the one at the beginning. Now, the beam is more or less along the longitudinal direction, but might be scattered in the traverse directions due to the energy loss/gain at the sample. Then, the longitudinal polarization of the beam will be analyzed at the end of the process by an analyzer whose transmission coefficient along the beam direction is stronger. Finally, an array of detectors is used to count analyzed neutron beam. Here, the measured neutron intensity $(P)$ can be formulated as: 


$$
\begin{gathered}
P=\left\langle\cos \left(\phi_{t o t}\right)\right\rangle=\left\langle\cos \left(\frac{\gamma H l}{m v^{3}} \hbar \omega\right)\right\rangle=\langle\cos (\omega t)\rangle \\
P=\frac{\int S(Q, \omega) \cos (\omega t) d \omega}{\int S(Q, \omega) d \omega}=\frac{I(Q, t)}{I(Q . t=0)}
\end{gathered}
$$

Thus, we can directly measure the normalized intermediate function, $I(Q, t) / I(Q, 0)$, and $t=\gamma H l / m v^{3}$ is the corresponding Fourier time. However, this formalism is valid only in the limit of $\hbar \omega \ll m v^{2} / 2$ and a more systematic way of measuring $I(Q, t)$ is needed. Thus, in practice, we measure $P$ by changing the magnetic field of the second solenoid with respect to the first one. When the neutron energy transfer is significant, $P$ shows a damped cosine-like dependence (spin echo) as a function of phase factor $(\phi)$, which is defined as the difference between field integrals of the two solenoids:

$$
\phi=\int H^{\prime} d l^{\prime}-\int H d l
$$

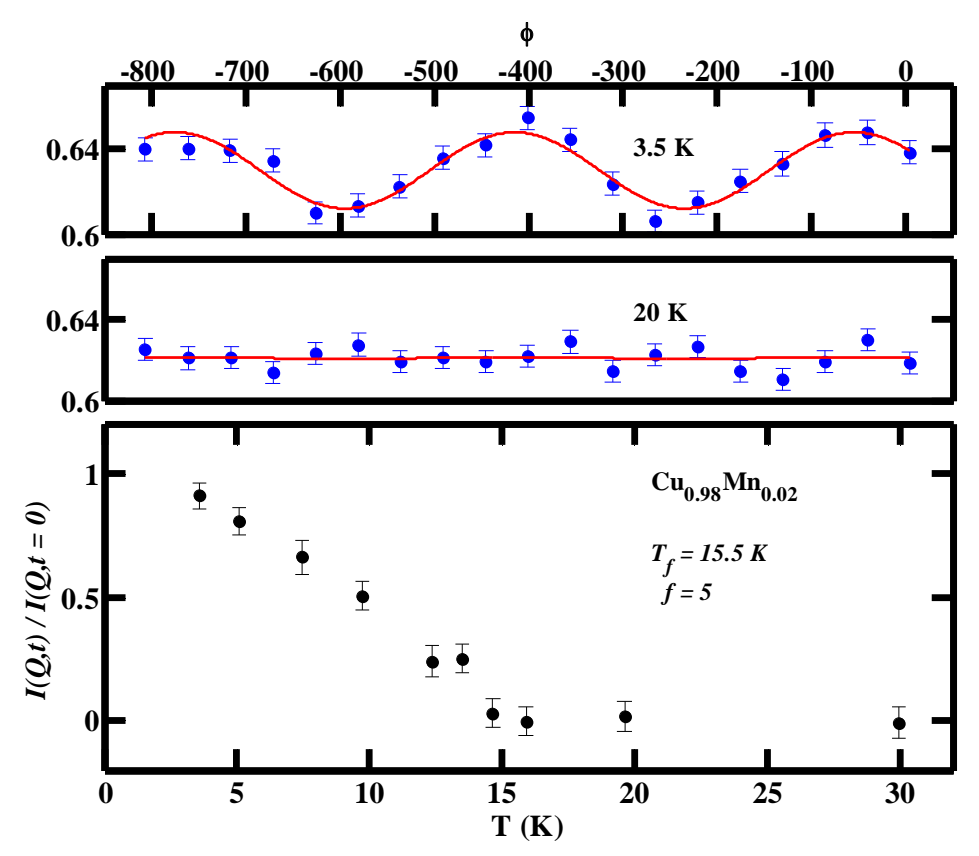

Figure 2.8 Neutron Spin Echo Order Parameter on $\mathrm{CuMn2 \%}$

The NSE measurement of $\mathrm{CuMn} 2 \%$ at (Top) $3.5 \mathrm{~K}$ and (Middle) $20 \mathrm{~K}$, which are below and above the spin glass transition of $15.5 \mathrm{~K}$, respectively. (Bottom) The normalized intermediate scattering function, $I(Q, t) / I(Q, 0)$ determined by the amplitude of the echo, as a function of temperature. Here, NSE measurements are corrected to the instrument resolution measured using a magnetic standard sample, $\mathrm{Ho}_{2} \mathrm{Ti}_{2} \mathrm{O}_{7}$. 
Figure 2.8 shows NSE measurements of a conventional spin glass material, $C u \mathrm{Mn} 2 \%$, as a function of temperature at Fourier time $5 \mathrm{~ns}$. Below the spin glass transition $T_{f}=15.5 \mathrm{~K}$, there are magnetic relaxations slower than $5 \mathrm{~ns}$ as the neutron spin echo dependence was clearly observed. (see the top panel of Figure 2.8) But, in the high temperature regime where all the relaxations are much faster than $5 \mathrm{~ns}$, the NSE measurement was flat as a function of $\phi$. (see the middle panel of Figure 2.8) The spin echo dependence is usually fitted by the following function,

$$
\mathrm{P}=\mathrm{P}_{0}+A \exp \left[\frac{\left(\phi-\phi_{0}\right)^{2}}{2 \sigma^{2}}\right] \cos \left[\frac{360}{T}\left(\phi-\phi_{0}\right)\right]
$$

where $A$ is the amplitude, $T$ is the period of the cosine function, and $\sigma$ is the width of the Gaussian envelop. The amplitude $(A)$ of the spin echo is proportional to the intermediate scattering function, $I(Q, t)$. Unlike the TOF scattering techniques, the spatial $(Q)$ and temporal $(t)$ information obtained from NSE experiments are completely decoupled and hence $I(Q, t)$ can be written as:

$$
I(Q, t)=I(Q t=0) \cdot s(t)
$$

where $I(Q, t=0)$ is the static structure factor and is usually estimated by a simple polarization analysis. In the case of non-magnetic experiments, determination of $I(Q, t=0)$ could be a simple exercise as measuring non-spin flip counts $\left(N_{N S F}\right)$ and spin flip counts $\left(N_{S F}\right)$ of neutrons by turning on and off the $\pi$-flipper without operating two solenoidal electromagnets. But in magnetic scattering experiments, three-directional neutron polarization measurements have to be performed using a XYZ-coil set, which is three pairs of orthogonal coils installed around the sample space. The experimental $I(Q, t=0)$ signal generally comes from nuclear coherent scattering, nuclear spinincoherent scattering and inefficiency of the instrument (polarizer, analyzer and flippers), as well as magnetic relaxations of the sample. Thus, a polarization analysis can be performed using six XYZ measurements (includes non-spin flip and spin flip count on each polarization direction) to estimate the magnetic contribution of $I(Q, t=0)$ as explained in Appendix E . Moreover, measured $I(Q, t)$ can be easily corrected for instrument resolution just by division as a consequence of being the Fourier transform of $S(Q, \omega)$. For a magnetic 
experiment, the instrument resolution is measured using a magnetic standard sample (usually $\mathrm{Ho}_{2} \mathrm{Ti}_{2} \mathrm{O}_{7}$ ) at base temperature.

\subsection{Spin Dynamics Simulations}

In order to study the dynamics of a given microscopic magnetic model, we consider the stochastic Landau-Lifshitz (LL) equation (Langevin equation) which describes the motion of a spin at position $i\left(S_{i}\right)$ :

$$
\dot{S}_{i}\left(\{S\}_{i j}\right)=\frac{d S_{i}}{d t}=\gamma\left[S_{i} \times F_{i}\right]-\gamma \alpha\left[S_{i} \times\left(S_{i} \times F_{i}\right)\right] ; \quad F_{i}=H_{i}\left(\{S\}_{i j}\right)+\xi_{i}(t)
$$

where, $F_{i}$ is the effective local field described by the microscopic Hamiltonian $H_{i}=$ $\frac{\partial H}{\partial S_{i}}$ and a stochastic field $\xi_{i}(t)$ with the following properties,

$$
\left\langle\xi_{i}(t)\right\rangle=0, \quad\left\langle\xi_{i}(t) \xi_{j}\left(t^{\prime}\right)\right\rangle=\frac{2 \alpha k_{B} T}{\gamma \mu_{s}} \delta\left(t-t^{\prime}\right) \delta_{i j}
$$

Here $T$ is the temperature, $\gamma$ is the gyromagnetic ratio, $\mu_{s}$ is the magnetic moment, and $\alpha$ is the parameter describing the coupling to the heat bath of the system (phonon or electron system). The first term in Equation 2.22 is just the equation of motion of a spin $S_{i}$ in a local field $F_{i}$, while the second term is the damping term which is in accordance with the fluctuation-dissipation theorem. Equation 2.22 performs well on the systems in which its heat bath acts much faster than the spin systems. The bath degree of freedom can be replaced by a stochastic field with a white noise correlation function, and the bath coupling coefficient $(\alpha)$ is determined by the fluctuation dissipation theorem [31]. Basically, the magnetization energy of the spin system is not conserved and will end up in an equilibrium state between coupling energy to heat bath and the ground state energy defined by its spin Hamiltonian. Thus, the final energy of the system depends on the temperature of the heat bath and the coupling constant.

When the time scale of the magnetic relaxations becomes comparable to the electron relaxations, the assumption is not valid anymore, and it is necessary to redefine the stochastic field and the coupling constant. For strongly correlated systems, the phonon density is considerably low, and the electron system is pretty much frozen in time, or it has 
very slow relaxations comparing to its magnetic counterpart in the low-temperature regime. Thus, it is reasonable to consider such a spin system as entirely decoupled from the heat bath $(\alpha \rightarrow 0)$.

To compare with neutron scattering measurements, we need to calculate the dynamical structure factor, $S(Q, \omega)$, for a given microscopic model at a given temperature. As the first step, we solve Equation 2.22 using the $4^{\text {th }}$ order Runge-Kutta method:

$$
\begin{gathered}
S_{i}(t+\delta t)=S_{i}(t)+\frac{\delta t}{6}\left(k_{1}+2 k_{2}+2 k_{3}+k_{4}\right)+\vartheta\left(\delta t^{5}\right) \\
k_{1}=\dot{S}_{i}\left(\{S\}_{i j}\right) ; \quad k_{2}=\dot{S}_{i}\left(\{S\}_{i j}+\frac{k_{1}}{2}\right) ; \quad k_{3}=\dot{S}_{i}\left(\{S\}_{i j}+\frac{k_{2}}{2}\right) ; \quad k_{4}=S_{i}\left(\{S\}_{i j}+k_{3}\right)
\end{gathered}
$$

where $\delta t$ is the time interval between two consecutive MC steps and a value for $\delta t$ has to be defined to keep the system energy conserved throughout the entire simulation. A smaller value of $\delta t$ is usually better as it assures to maintain energy conservation, yet it costs more computational time. Thus, it is important to determine a proper value for $\delta t$ in which the interaction energies of the Hamiltonian and the spin size (S) are the main factors. The length of the simulation (total MC steps) and the sampling rate of spin configurations depend on the required $\omega$-resolution and maximum frequency $\left(\omega_{\max }\right)$ respectively.

Usually, each simulation is comprised of two consecutive processes, thermalization and dynamics. In the thermalization process, we used standard metropolis sampling on local magnetic energy to find a thermally stable configuration for a given temperature, starting from a completely random configuration. Once the system is thermally stable, a few percent of randomly selected spins in the final configuration would be flipped to emulate the neutron probing effect. Then, the spin dynamic simulation step starts, and the final configuration from the thermalization step would be used as the initial condition to solve Equation 2.22 numerically. In order to calculate the dynamical structure factor $S(Q, \omega)$, we perform Fourier transform on the real space and time information $\left\{S_{i j}(t)\right\}$ as explained below. 
A simplified version of the dynamic structure factor can be written as:

$$
\begin{aligned}
S(\vec{Q}, \omega)= & \sum_{\alpha \beta} \sum_{j k} \sum_{t, t^{\prime}} \exp \left[-i \vec{Q} \cdot\left(\vec{r}_{j}-\vec{r}_{k}\right)\right] \exp \left[-i \omega\left(t-t^{\prime}\right)\right]\left\langle S_{j}^{\alpha}\left(\vec{r}_{j}, t\right) S_{k}^{\beta}\left(\vec{r}_{k}, t^{\prime}\right)\right\rangle \\
& =\sum_{\alpha \beta} I^{\alpha} \times\left(I^{\beta}\right)^{\dagger}
\end{aligned}
$$

With neutron polarization factor,

$$
S(Q, \omega)=\sum_{\alpha \beta}\left\{\delta_{\alpha \beta}-\hat{k}_{\alpha} \hat{k}_{\beta}\right\} . I^{\alpha} \times\left(I^{\beta}\right)^{\dagger}
$$

where,

$$
I^{p}(Q, \omega)=\sum_{j} \exp \left[-i Q . \vec{r}_{j}\right] I_{j}^{p}\left(\vec{r}_{j}, \omega\right) ; I_{j}^{p}\left(\vec{r}_{j}, \omega\right)=\sum_{t} \exp [-i \omega(t)] S_{j}^{p}\left(\vec{r}_{j}, t\right)
$$

In Chapter 8 , we have used Equation 2.25 to calculate $S(Q, \omega)$. In order to compare $S(Q, \omega)$ with neutron scattering experiments, we need to include the magnetic form factors, $F(Q)$, of the corresponding magnetic species to our magnetic structure factor calculations:

$$
\tilde{S}(Q, \omega)=\left\{\frac{1}{2} g F(Q)\right\}^{2} \times S(Q, \omega)
$$

The full scattering cross-section for a spin system can be evaluated as shown below: [23]

$$
\frac{d^{2} \sigma}{d \Omega d E^{\prime}}=r_{0} \frac{k_{f}}{k_{i}}\left\{\frac{1}{2} g F(Q)\right\}^{2} \exp [-2 W(Q)] \times S(Q, \omega) \times \delta\left(E^{\prime}-E-\hbar \omega\right) ; \quad k_{f}=k_{i}+Q
$$

And the spin-spin correlation function can be calculated by,

$$
S(r, \omega)=\sum_{p} \sum_{r=\left|r_{j k}\right|} I_{j}^{p} \times\left(I_{k}^{p}\right)^{\dagger} ; \quad r_{j k}=r_{j}-r_{k}
$$

Thus, the on-site component $S_{0}$, and nearest neighbor component $S_{1}$ are:

$$
\begin{aligned}
& S_{0}(\omega)=\sum_{p} \sum_{j}\left\langle S_{j}^{p}(\omega)\right\rangle \times\left\langle S_{j}^{p}(\omega)\right\rangle^{\dagger} ; \\
& S_{1}(\omega)=\sum_{p} \sum_{l_{j k}}\left\langle S_{l_{j}}^{p}(\omega)\right\rangle \times\left\langle S_{l_{k}}^{p}(\omega)\right\rangle^{\dagger} ;
\end{aligned}
$$




\section{Chapter 3}

\section{A Quasi-Two-Dimensional Magnetic System}

\subsection{Motivation}

Since the low-temperature spin glass phase was first found in $A u$ Co binary alloy, numerous experimental and theoretical investigations have been done to characterize this anomalous phase. Subsequently, magnetic glass behavior was observed in many other magnetic materials ranging from geometrically frustrated magnets to superconductors. Some glass phases found in frustrated magnets shows unconventional spin-glass behaviors displaying nonergodic nature in the bulk susceptibility [32] and short-range spin correlations but with a strongly momentum-dependent structure factor in neutron scattering [33]. Among many frustrated magnets, $\operatorname{SrCr}_{9 p} \mathrm{Ga}_{12-9 p} \mathrm{O}_{19}(\mathrm{SCGO}(p))$ [33--41] and $\mathrm{Ba}_{2} \mathrm{Sn}_{2} \mathrm{ZnGa}_{3} \mathrm{Cr}_{7} \mathrm{O}_{22}$ (BSZGCO) $[39,42--44]$ are particularly interesting due to glassiness obtained from a quasitwo-dimensional lattice in which the magnetic $\mathrm{Cr}^{3+}\left(3 d^{3} ; s=3 / 2\right)$ ions form a kagometriangular-kagome trilayer. Moreover, both of these systems have higher degree of frustration as estimated by the frustration index, $f=\left|\theta_{c w}\right| / T_{f}>100$. At this limit of frustration, a spin liquid is usually expected in the mean-field level, and it is inconsistent with the observed glass-like properties of these materials at low temperature (T).

Since the discovery of SCGO more than two decades ago [35], numerous experimental investigations have been performed to study its anomalous low temperature phase including bulk susceptibility $[32,35,45]$, specific heat $[32,37]$, muon spin relaxation $(\mu \mathrm{SR})$ [38], nuclear magnetic resonance [41,46], and neutron scattering [33]. A nonergodic nature 
from Zero-field-cooled (ZFC) and field-cooled (FC) hysteresis was observed similar to the conventional spin glasses along with non-spin-glassy behaviors, such as a quadratic dependence of specific heat at low $T, C_{v} \propto T^{2}$ [37], a linear dependence of the imaginary part of the dynamic susceptibility at low energies, $\chi^{\prime \prime}(\omega) \propto \omega$ [34], and a broad but prominent momentum dependence of the elastic neutron scattering intensity [34]. The theoretical realization of the freezing mechanism and low- $T$ frozen state of SCGO has been a challenging problem in condensed matter physics. To review some of existing theoretical developments, a spin liquid with unconfined spinons or resonating valence bond state was proposed based on NMR and $\mu$ SR studies $[38,46]$, and many-body singlet excitations were also suggested to be responsible for the $C_{v} \propto T^{2}$ behavior [37].

Recently, an alternative scenario involving a spin jam state due to quantum fluctuations was proposed $[4,5]$. The spin jam of disorder free SCGO provided a qualitatively coherent understanding of all of the low-temperature behaviors including the complex energy landscape responsible for the frozen state [5], and Halperin-Saslow (HS)-like modes for the $C_{v} \propto T^{2}$ and $\chi^{\prime \prime}(\omega) \propto \omega$ behaviors $[5,15]$. The materials that can clearly realize the spin jam state are rare, such as $\operatorname{SCGO}(p)$ and BSZGCO. More recently, a new quasi-twodimensional magnet $\mathrm{BaCr}_{9 p} \mathrm{Ga}_{12-9 p} \mathrm{O}_{19}[\mathrm{BCGO}(p)]$ isostructural to $\mathrm{SCGO}(p)$ by replacing Sr by Ba, was synthesized. [47] In this chapter, we discuss the structure and experimental signatures of two spin jam materials, $\operatorname{SCGO}(p)$ and $\operatorname{BCGO}(p)$. Starting from basic characterization using bulk susceptibility, we discuss both quasi-elastic and inelastic neutron scattering experiments.

\subsection{Crystal Structure}

Neutron powder diffraction experiments were performed on $\operatorname{SCGO}(p)$ and $\operatorname{BCGO}(p)$ samples summarized in Table 3-1 at High-Resolution Powder Diffractometer (BT01), National Institute of Standards and Technology (NITS). Subsequent Rietveld refinements on powder diffraction data confirmed that SCGO and BCGO systems are isostructural and consistent with the previously published structure of $\operatorname{SCGO}(p=0.925)$ [34]. Furthermore, all the diffraction patterns could be indexed by a single phase such that overall reduced $\chi^{2}$ of refinements ranges from 2.1 to 4.9. (see Appendix A.4 for $\operatorname{SCGO}(p)$ and Appendix A.5 for $\operatorname{BCGO}(p))$. 
As shown in Figure 3.1 (a), the $\mathrm{Cr}^{3+}$ ions in $\operatorname{SCGO}(p) / \mathrm{BCGO}(p)$ occupy in three crystallographically distinct sites, denoted $12 k, 2 a$ and $4 f_{v i}$, respectively. [34] The $12 k$ site forms a slightly distorted kagome layer, whereas the $2 a$ site forms a triangular lattice. The $12 k-2 a-12 k$ block forms a triangular network of bipyramids. The $4 f_{v i} \mathrm{Cr}^{3+}$ ions sit in between the Quasi 2D layers and $4 f_{v i}-4 f_{v i} \mathrm{Cr}^{3+}$ ions are likely to form spin dimers. $[34,36]$ The full structure of BCGO/SCGO is obtained by the stacking of spin dimers and the triangular network of bipyramids. Each $\mathrm{Cr}^{3+}$ ion is surrounded by a distorted octahedron of oxygen atoms. Oxygen octahedra of chromium ions in adjacent $4 f_{v i}$ planes share a common face, whereas the oxygen octahedra of neighboring $4 f_{v i}$ and $12 k$ sites share a corner. For clarity, only selected oxygen atoms are shown in Figure 3.1 (b).
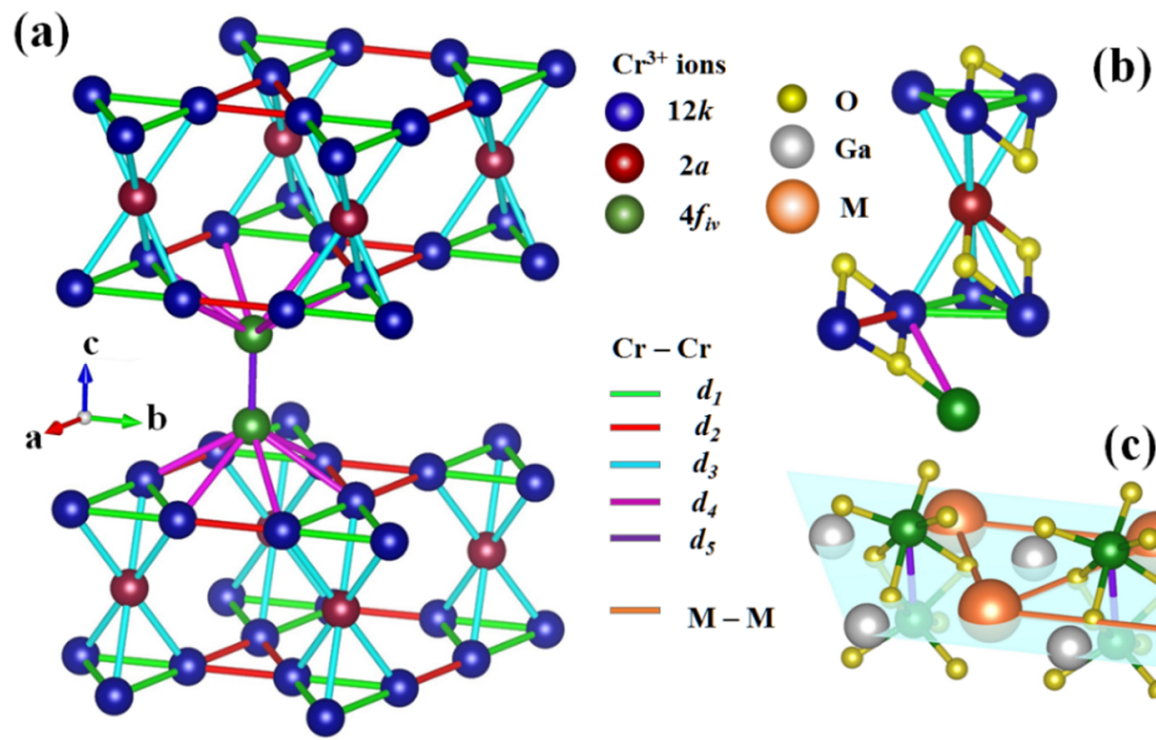

\section{(c)}

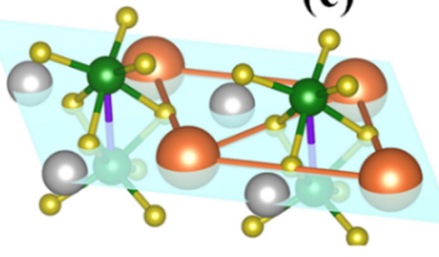

Figure 3.1 The Crystal Structure of SCGO/BCGO system.

(a) The magnetoplumbite structure of $\operatorname{SCGO}(p) / \operatorname{BCGO}(p)$ in which $\mathrm{Cr}^{3+}$ ions are arranged in three different crystallographic sites, $2 a, 12 k$ and $4 f_{i v}$. The $12 k-2 a-12 k$ trilayers forms a triangular network of bipyramids and five different nearest neighbor (NN) bonds can be considered. (b) A block of $\mathrm{Cr}^{3+}$ ions showing a closer illustration of different $\mathrm{NN}$ bonds. Each $\mathrm{Cr}^{3+}$ is surrounded by a distorted octahedron of oxygen atoms. Here only selected oxygen atoms are shown for clarity. (c) The $4 f_{i v}-4 f_{i v}$ layer in which $\mathrm{Cr}^{3+}$ ions are paired by face sharing octahedral ligand environments and forms spin-dimers. The $\mathrm{Cr}^{3+}$ dimers, $\mathrm{M}(\mathrm{Sr}$ or $\mathrm{Ba})$ atoms and $\mathrm{Ga}$ atoms are arranged in triangular networks separately in such that $\mathrm{M}$ and $\mathrm{Ga}$ resides at the centers of neighboring triangle of spin dimers.

The non-magnetic $\mathrm{M}(\mathrm{Ba}$ or $\mathrm{Sr})$ atoms reside in a triangular lattice between the $4 f_{v i} \mathrm{Cr}^{3+}$ layers as shown in Figure 3.1 (c). In the clean limit ( $p \rightarrow 1)$, the non-magnetic Ga atoms 
reside in two distinct crystallographic sites, denoted by $2 b$ and $4 f$, only. But the Ga atoms are more or less equally occupied in the Cr sublattices $\left(12 k, 2 a\right.$ and $\left.4 f_{v i}\right)$ in all the other doping levels $(p<1)$. (see Appendix A.4 and Appendix A.5 )

The lattice constants, true magnetic ion concentrations $(p)$ and the $\mathrm{Cr}^{3+}-\mathrm{Cr}^{3+}$ bond distances for $\operatorname{SCGO}(p) / \mathrm{BCGO}(p)$ samples are summarized in Table 3-1 in comparison to $\operatorname{SCGO}(p=$ 0.925) [34] and BSZCGO [48]. As shown in Figure 3.1 (a), the bonding distance $d_{5}$ is defined as the length of the $4 f_{v i}-4 f_{v i}$ spin dimer, $d_{1}$ and $d_{2}$ are the bonding distances in the $12 k$ kagome layer, and $d_{4}$ is the bonding distance of $12 k-2 a$.

Table 3-1 Comparison of lattice constants and bond distances between $\mathrm{Cr}^{3+}$ ions in SCGO/BCGO As shown in Figure 3.1(a), $d 1$ is the bonding distance of $4 f_{v i}-4 f_{v i}$ spin dimer, $d 2$ and $d 3$ are the bonding distances in $12 k$ kagome layer, and $\mathrm{d} 4$ is the bonding distance of $12 k-2 a$, respectively. $a$ and $c$ are the lattice parameters.

\begin{tabular}{|c|c|c|c|c|c|c|}
\hline Sample & $d 5(\AA)$ & $d \mathbf{d 1}(\AA)$ & $d 2(\AA)$ & $d 3(\AA)$ & $a(\AA)$ & $\overline{c(\AA)}$ \\
\hline $\operatorname{SCGO}(p=0.968(6))$ & $2.685(8)$ & $2.863(6)$ & $2.930(6)$ & $2.982(3)$ & $5.7948(3)$ & $22.6625(2)$ \\
\hline $\operatorname{SCGO}(p=0.844(8))$ & $2.696(8)$ & $2.862(5)$ & $2.933(5)$ & $2.997(3)$ & $5.79447(3)$ & $22.7120(2)$ \\
\hline $\operatorname{SCGO}(p=0.620(8))$ & $2.706(6)$ & $2.871(4)$ & $2.926(4)$ & $3.007(2)$ & $5.79676(3)$ & $22.7541(2)$ \\
\hline $\operatorname{SCGO}(p=0.459(5))$ & $2.751(9)$ & $2.863(6)$ & $2.932(6)$ & $3.015(3)$ & $5.79566(3)$ & $22.7778(2)$ \\
\hline $\operatorname{SCGO}(p=0.214(5))$ & $2.746(5)$ & $2.866(3)$ & $2.927(3)$ & $3.014(1)$ & $5.79342(7)$ & $22.8033(3)$ \\
\hline $\operatorname{BCGO}(p=0.902(8))$ & $2.727(5)$ & $2.878(3)$ & $2.926(3)$ & $2.973(2)$ & $5.8037(4)$ & $22.8483(3)$ \\
\hline $\operatorname{BCGO}(p=0.806(8))$ & $2.752(9)$ & $2.885(9)$ & $2.919(7)$ & $2.978(8)$ & $5.8057(3)$ & $22.8726(6)$ \\
\hline $\operatorname{BCGO}(p=0.712(7))$ & $2.758(4)$ & $2.878(9)$ & $2.927(5)$ & $2.990(5)$ & $5.8064(8)$ & $22.8897(4)$ \\
\hline $\operatorname{BCGO}(p=0.635(7))$ & $2.761(4)$ & $2.886(0)$ & $2.920(9)$ & $2.994(4)$ & $5.8069(9)$ & $22.9074(3)$ \\
\hline $\operatorname{BCGO}(p=0.526(5))$ & $2.792(1)$ & $2.886(3)$ & $2.920(8)$ & $2.999(7)$ & $5.8071(9)$ & $22.9190(7)$ \\
\hline $\operatorname{BCGO}(p=0.417(7))$ & $2.807(1)$ & $2.878(1)$ & $2.928(5)$ & $3.002(5)$ & $5.8066(2)$ & $22.9317(3)$ \\
\hline $\operatorname{SCGO}(p=0.925)^{\mathrm{a}}$ & 2.67 & 2.87 & 2.92 & 2.98 & $5.7954(1)$ & $22.6446(6)$ \\
\hline BSZCGO $^{\text {b }}$ & - & $2.885(6)$ & $2.971(1)$ & $2.963(0)$ & $5.8568(1)$ & $14.2537(3)$ \\
\hline
\end{tabular}

${ }^{\mathbf{a}}$ From Ref. [34] . ${ }^{\mathbf{b}}$ From Ref. [48].

Comparing $\operatorname{BCGO}(p=0.902(8))$ with $\operatorname{SCGO}(p=0.925)$, the increase in the $a$-axis lattice constant is $(\sim 0.1 \%, 0.0083 \AA)$, significantly smaller than that of the c-axis lattice constant $(\sim 0.9 \%, 0.2037 \AA)$, which leads to the reduced dimensionality in $\operatorname{BCGO}(p=0.902(8))$. Furthermore, the bonding distances in the triangular network of bipyramids $(d 1, d 2$ and $d 3)$ of $\operatorname{BCGO}(p=0.902(8))$ are almost the same as for $\operatorname{SCGO}(p=0.925)$ (difference 
smaller than $0.008 \AA)$, but the bonding distance for spin dimers $\left(d_{5}\right)$ in $\operatorname{BCGO}(p=$ $0.902(8))$ is obviously larger than that in $\operatorname{SCGO}(p=0.925)$ with an increase of $0.057 \AA$. The increase in the $c$-axis and $d_{1}$ can be understood by the bigger atomic radius of $\mathrm{Ba}$. As shown in Figure 3.1(c), Ba or $\mathrm{Sr}$ reside in between the $4 f_{v i} \mathrm{Cr}^{3+}$ layers, the substitution of $\mathrm{Sr}$ by the bigger $\mathrm{Ba}$ atom will increase $d_{5}$ and further elongate the c-axis. Since $\mathrm{Ba}$ atoms do not occupy positions in the triangular network of bipyramids, the distances $d_{1}, d_{2}$, and $d_{3}$, as well as the $a$-axis length are barely changed. Meanwhile, as we can see from Table $3-1, d_{5}$ and the $c$-axis significantly increase with increasing Ga doping level (decreasing $p$ value), and $d_{4}$ slightly increases but $d_{1}, d_{2}$ and the length of the $a$-axis barely change.

As we observed from the refinement results of the neutron powder diffraction data, substitution of Sr by Ba leads to a significantly elongated $c$-axis but the $a$-axis as well as bonding distance of $\mathrm{Cr}^{3+}$ in the kagome plane are barely changed. Since the $\mathrm{Cr}^{3+}-\mathrm{Cr}^{3+}$ magnetic interactions are mainly due to the direct overlap of the $t_{2 g}$ orbitals, the coupling constant is known to be sensitive to the distance between the neighboring $\mathrm{Cr}^{3+}$ ions. Thus, the magnetic interaction in $\mathrm{BCGO}(p)$ is expected to be stronger in the kagome plane than that of the layered triangular interplane, hence to be more two dimensional than in $\operatorname{SCGO}(p)$.

\subsection{Bulk Susceptibility Experiments}

We have measured DC magnetic susceptibilities, on both $\operatorname{SCGO}(p)$ and $\operatorname{BCGO}(p)$ samples in the temperature range from $20 \mathrm{~K}$ to $350 \mathrm{~K}$. (see Figure 4.2 for $\operatorname{SCGO}(p)$ and see Figure (App.) A.1 for $\operatorname{BCGO}(p)$ ). All the $1 / \chi-T$ curves show clear linear dependences above $150 \mathrm{~K}$ with similar values for the slope indicating that the Curie Constants $\mathrm{C}$ and effective moment per $\mathrm{Cr}^{3+}\left(\mu_{e f f}\right)$ are $p$-independent. $\mu_{e f f}$ is $\sim 4.1 \mu_{B} / \mathrm{Cr}^{3+}$, close to the value expected for $s=3 / 2$ which is $3.87 \mu_{B}$. Moreover, the Curie-Weiss temperatures $\theta_{c w}$, extracted from the intercepts of linear fits to $1 / \chi-T$ data, are comparatively large reflecting the strong AFM interactions in these magnets. For example, $\left|\theta_{c w}\right| \sim 504.6 \mathrm{~K}$ and $695 \mathrm{~K}$ for $\operatorname{SCGO}(p=0.968(6))$ and $\operatorname{BCGO}(p=0.902(8))$ samples respectively. (see Table (App.) A-9) 

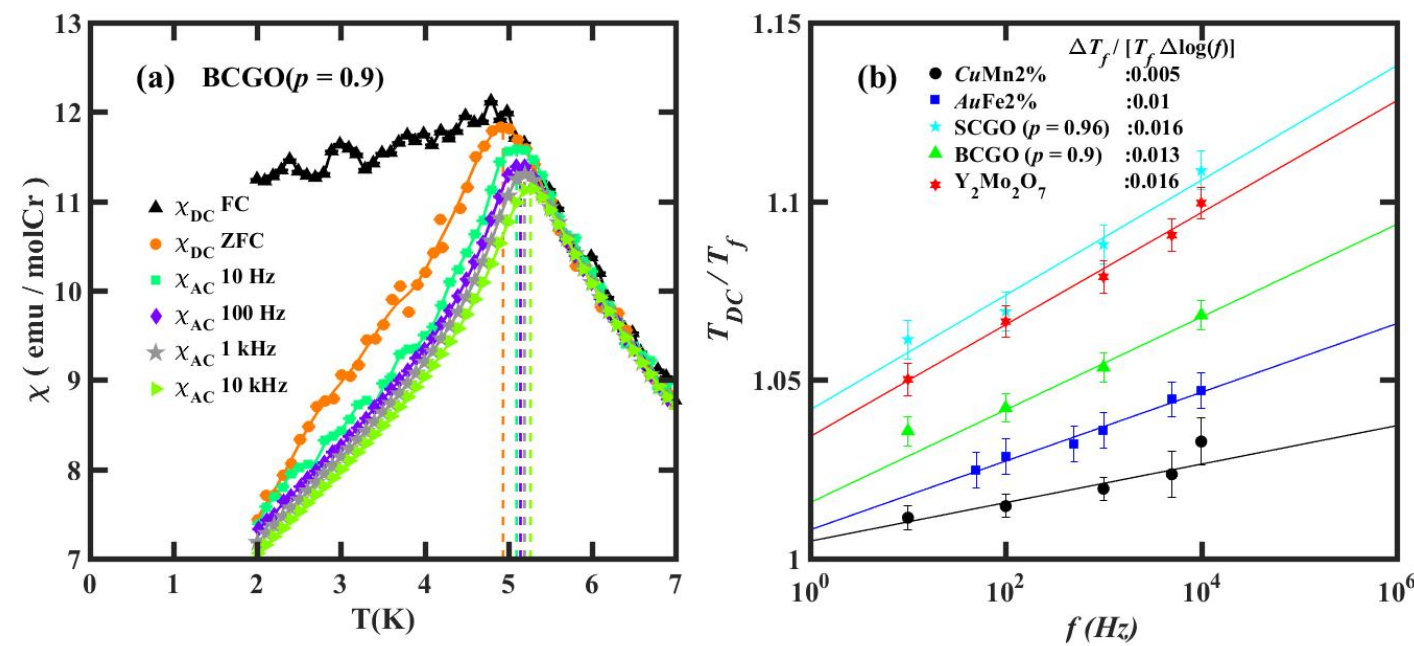

Figure 3.2 Bulk susceptibility measurements of $\operatorname{BCGO}(p=0.902(8))$

(a) The bulk susceptibility measurements of $\operatorname{BCGO}(p=0.902(8))$ sample including ZFC/FC DC magnetization measurements and AC magnetization measurements at four different probing frequencies $(f)$. The dash lines mark the cusp temperature $\left(T_{f}\right)$ of each magnetization curve. (b) $T_{D c} / T_{f}$ as a function of $\log (f)$ for $\operatorname{BCGO}(p=0.902(8))$ along with four other magnetic glass systems: $\operatorname{SCGO}(p=0.968(6)), C u \mathrm{Mn} 2 \%, A u \mathrm{Fe} 2 \%$ and $\mathrm{Y}_{2} \mathrm{Mo}_{2} \mathrm{O}_{7}$. The solid lines are the fits of $T_{f}$ data to the function, $\log \left(T_{f}\right)=m \log (f)+b$, where $m=\Delta T_{f} /\left(T_{f} \Delta \log (f)\right)$.

The low-temperature DC susceptibility measurements in the temperature range from $2 \mathrm{~K}$ to $20 \mathrm{~K}$ were performed while heating a sample followed by a continuous cooling process with or without an applied field of $0.01 \mathrm{~T}$. Most of the zero-field-cooled measurements shows a cusp at low temperatures below $5 \mathrm{~K}$ and the cusp temperature, $T_{D C}$ decreases as the number of spin vacancies increases and was undetectable at $\mathrm{Cr}$ concentrations as low as $p=0.526(7)$ in our available temperature range $(\geq 1.8 \mathrm{~K})$. Thus, both $\left|\theta_{c w}\right|$ and $T_{D C}$ show nearly linear and decreasing dependences while corresponding frustration indices $f=\left|\theta_{c w}\right| / T_{D C}$, show opposite behavior with increasing nonmagnetic Ga concentration. For example, the frustration index, $f$ increases from 140 to 195 for $\operatorname{BCGO}(p)$ while it increases from 135 to 155 for $\operatorname{SCGO}(p)$ as $p$ decreases. (see Figure (App.) A.4) The large frustration indices indicates that $\operatorname{BCGO}(p) / \operatorname{SCGO}(p)$ magnets are highly frustrated and the behaviors of $T_{D C}, \theta_{c w}$ and $f$, are consistent with the prediction of recent theoretical work on a spin jam, which is classically a liquid, freezing in the clean limit $(p \rightarrow 1)$ into a glassy state induced by quantum fluctuations. [4,5,49] 
Moreover, all the systems except $\operatorname{SCGO}(p=0.214(5))$, show a bifurcation between zero-field cooled (ZFC) and field-cooled(FC) magnetization measurements below a finite temperature $T_{\text {irr }}$, implying a magnetic transition to a non-ergodic phase from a high temperature ergodic phase. (see Figure 4.3 and Figure (App.) A.1 (b))For example, the ZFC-FC hysteresis were observed below the freezing temperature $T_{D C} \approx 5 \mathrm{~K}$ in the case of $\operatorname{BCGO}(p=0.902(8))$ as shown in Figure 3.2 (a) and it is consistent with both previously published SCGO data [32,35,37,50] and the typical magnetic glass nature [7].

The dilution effects of the $\operatorname{SCGO}(p) / \mathrm{BCGO}(p)$ is not the primary focus of this Chapter instead study the magnetic glassy nature of $\operatorname{BCGO}(p) / \operatorname{SCGO}(p)$ in the clean limit $(p \rightarrow 1)$. However, we will discuss dilution effects of $\operatorname{SCGO}(p)$ extensively in Chapter 4 . Most of $\operatorname{SCGO}(p) / \mathrm{BCGO}(p)$ samples at higher values of $p$ contains a second phase of $\mathrm{Cr}_{2} \mathrm{O}_{3}$ which is one of the constituents used in the sample synthesis (see Appendix A.1 for more information). As verified by Neutron Diffraction measurements, $\operatorname{BCGO}(p=0.902(8))$ and $\operatorname{SCGO}(p=0.968(6))$ samples turned out to be a fairly clean with an undetectable amount of second phase, closer to the clean limit $(p \rightarrow 1)$. (see Appendix A.3 , A.4 , and A.5 ) Thus, we considered the $\operatorname{BCGO}(p=0.902(8))$ and $\operatorname{SCGO}(p=0.968(6))$ samples as potential candidates of the spin-jam and used them to study the low-temperature phase extensively using bulk susceptibility and neutron scattering spectroscopy. In the rest of the chapter, we will mainly focus on the experimental signatures of $\operatorname{BCGO}(p=0.902(8))$.

The AC susceptibility, $\chi_{A C}$ has also been measured on $\operatorname{BCGO}(p=0.902(7))$ sample at different frequencies $(f)$ values of applied AC field and zero static field conditions. As shown in the Figure 3.2(a), $\chi_{A C}$ shows a cusp similar to the ZFC magnetization curve but the $\chi_{A C}$ cusp temperatures $\left(T_{f}\right)$ are shifted to higher temperatures with increasing frequency. This behavior is an indication of having multiple relaxation time scales at the vicinity of the magnetic transition defined by $T_{D C}$ and can be usually found in Spin Glasses, Cluster-Spin Glasses or Superparamagnets. The parameter, $\Delta T_{f} /\left(T_{f} \Delta \log (f)\right)$ is generally used to distinguish different magnetic phases. [7] In Figure $3.2(\mathrm{~b})$, the $T_{D C} / T_{f}$ is been plotted as a function of $\log (f)$ and the parameter, $\Delta T_{f} /\left(T_{f} \Delta \log (f)\right)$, can be directly extracted as the slope of the linear fits. 
As summarized in the Figure 3.2 (b) legend, the $\operatorname{BCGO}(p=0.902(8)$ ) shows a slightly higher value for $\Delta T_{f} /\left(T_{f} \Delta \log (f)\right)$ in comparison to the archetypical spin glasses $C u \mathrm{Mn} 2 \%$ or $A u \mathrm{Fe} 2 \%$. This could be a signature of having a low temperature phase different from Spin Glass in the case of $\operatorname{BCGO}(p=0.902(8))$ and similar behavior is observed for the isostructural $\operatorname{SCGO}(p=0.968(6))$ and $\mathrm{Y} 2 \mathrm{Mo} 2 \mathrm{O} 7$, which is a three dimensional frustrated magnetic. The frequency dependence of $T_{f}$ will be discussed in much broader scale in Chapter 5 .

\subsection{Two-dimensionality of $\operatorname{BCGO}(p=0.902(8))$ and the isolated spin pairs}

To study magnetic excitations of $\mathrm{BCGO}(p=0.902(8))$, we performed inelastic neutron scattering (INS) measurements on using the Disk Chopper Spectrometer (DCS) at NIST Center for Neutron Research (NCNR) with an incident wavelength of $\lambda=1.5 \AA$ and in the temperature range from $1.5 \mathrm{~K}$ to $300 \mathrm{~K}$.

Figure 3.3 (a) and Figure 3.3 (b) show contour maps of the neutron scattering intensity, $I(Q, \omega)$, at $\mathrm{T}=1.5 \mathrm{~K}$ and $192.7 \mathrm{~K}$, respectively. [51] As shown in Figure 3.3 (a) and Figure $3.3(\mathrm{~b}), I(Q, \omega)$ is dominated by two ridges, one at fixed wave-vector transfer, $Q \approx$ $1.5 \AA^{-1}$, and the other ridge at fixed energy transfer $\hbar \omega=16.5(1) \mathrm{meV}$, which are quite similar to what as observed in $\operatorname{SCGO}(p=0.925)$ [34]. The $\hbar \omega=16.5(1) \mathrm{meV}$ ridge is narrow in energy and non-dispersive as in $\operatorname{SCGO}(p=0.925)$, and likely to arise from $4 f_{v i}-$ $4 f_{v i}$ spin dimers. Further clues come from finite temperature properties and our fitting results. To enhance sensitivity, we plot in Figure 3.3 (c) and Figure 3.3 (d) the $Q$-integrated intensity which probes the local spin fluctuation spectrum: $I(\omega)=$ $\int I(Q, \omega)\left[\frac{Q}{\mid F(Q) 1}\right]^{2} d Q / \int Q^{2} d Q$. [52] Corresponding to the $\hbar \omega=16.5(1) \mathrm{meV}$ ridge shown in Figure $3.3(\mathrm{a})$, the $Q$-integrated intensity $I(\omega)$ at $\mathrm{T}=1.5 \mathrm{~K}$ clearly exhibits a peak at $\hbar \omega=16.5(1) \mathrm{meV}$ in Figure 3.3 (c). As shown in Figure 3.3 (d), at $\mathrm{T}=192.7 \mathrm{~K}$, the peak at $\hbar \omega=16.5(1) \mathrm{meV}$ is weak but still observable. For comparison, the $Q$-integrated intensity of $\operatorname{SCGO}(p=0.968(6))$ at $\mathrm{T}=1.5 \mathrm{~K}$ is also plotted in Figure $3.3(\mathrm{c})$, which shows a similar peak at higher energy: $\hbar \omega=18.6(1) \mathrm{meV}$. 

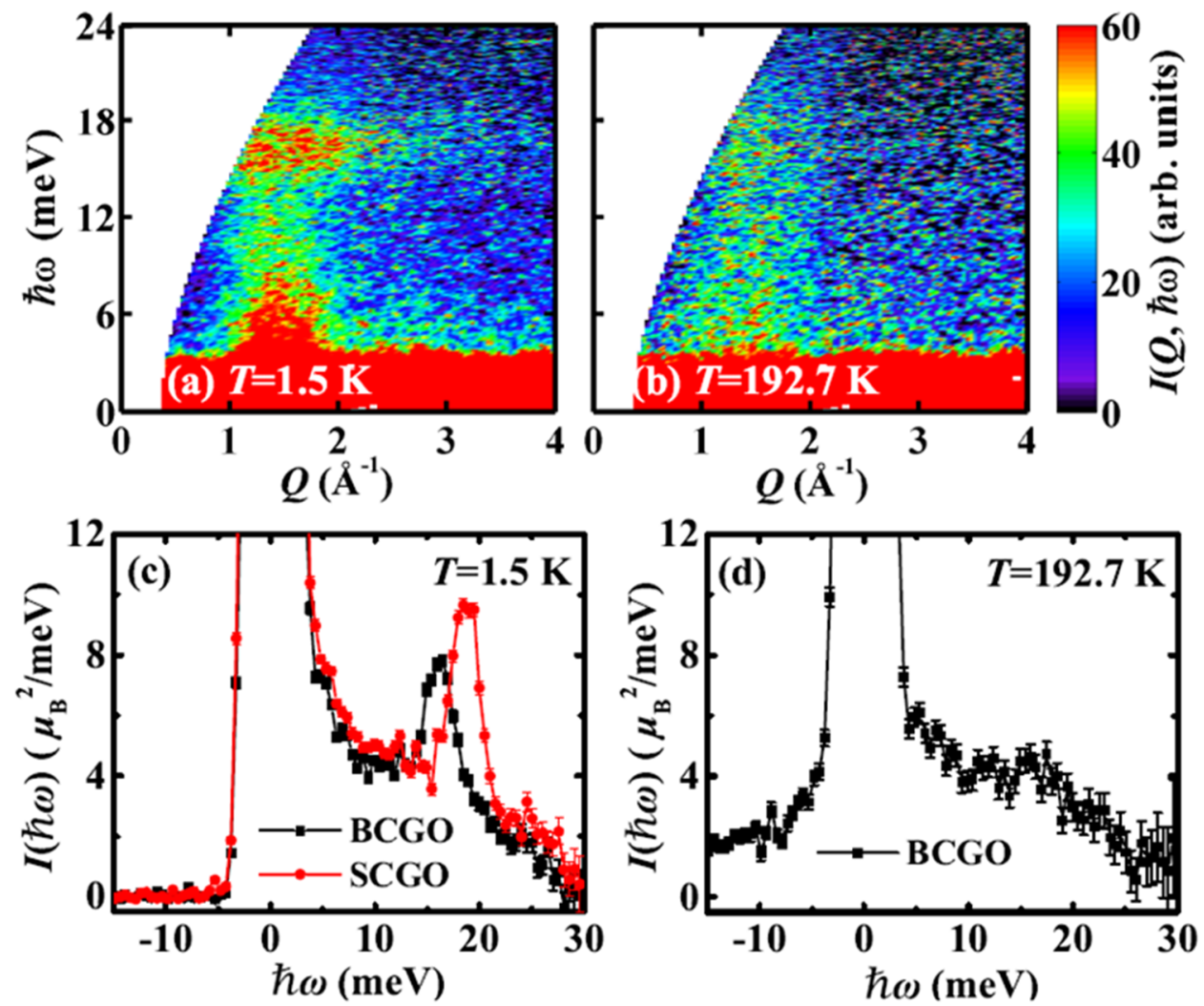

Figure 3.3 Inelastic Neutron Scattering of $\operatorname{BCGO}(p=0.902(8))$

Color image of neutron scattering intensity, $I(Q, \omega)$, in $\operatorname{BCGO}(p=0.902(8))$ at (a) $T=1.5 \mathrm{~K}$ and (b) $\mathrm{T}=192.7 \mathrm{~K} . \omega$ dependence of the Q-integrated neutron scattering intensity, $I(\omega)=$ $\int\left[I(Q, \omega) /|F(Q)|^{2}\right] Q^{2} d Q / \int Q^{2} d Q$ at (c) $T=1.5 \mathrm{~K}$ for $\operatorname{BCGO}(p=0.902(8))$ and $\operatorname{SCGO}(p=$ $0.925)$, and (d) $\mathrm{T}=192.7 \mathrm{~K}$ for BCGO $(p=0.902(8))$.

Figure 3.4 (a) and Figure 3.4 (b) summarize the $T$ dependence of the excitation energy $E_{0}$ and the resolution corrected full width at half maximum (FWHM) $\Delta E$ of the $16.5(1) \mathrm{meV}$ excitations. The elastic energy resolution is about $2 \mathrm{meV}$. These results were obtained by fitting the data, such as those in Figure 3.3 (c) and Figure 3.3 (d), with Gaussians. As shown in Figure 3.4 (a) and Figure 3.4 (b), $E_{0}$ is T independent and $\Delta E / E_{0}<0.2$ even for $k_{B} T>$ $E_{0}$, indicating that the excitations originate from well-defined quantum states of isolated spin clusters. 


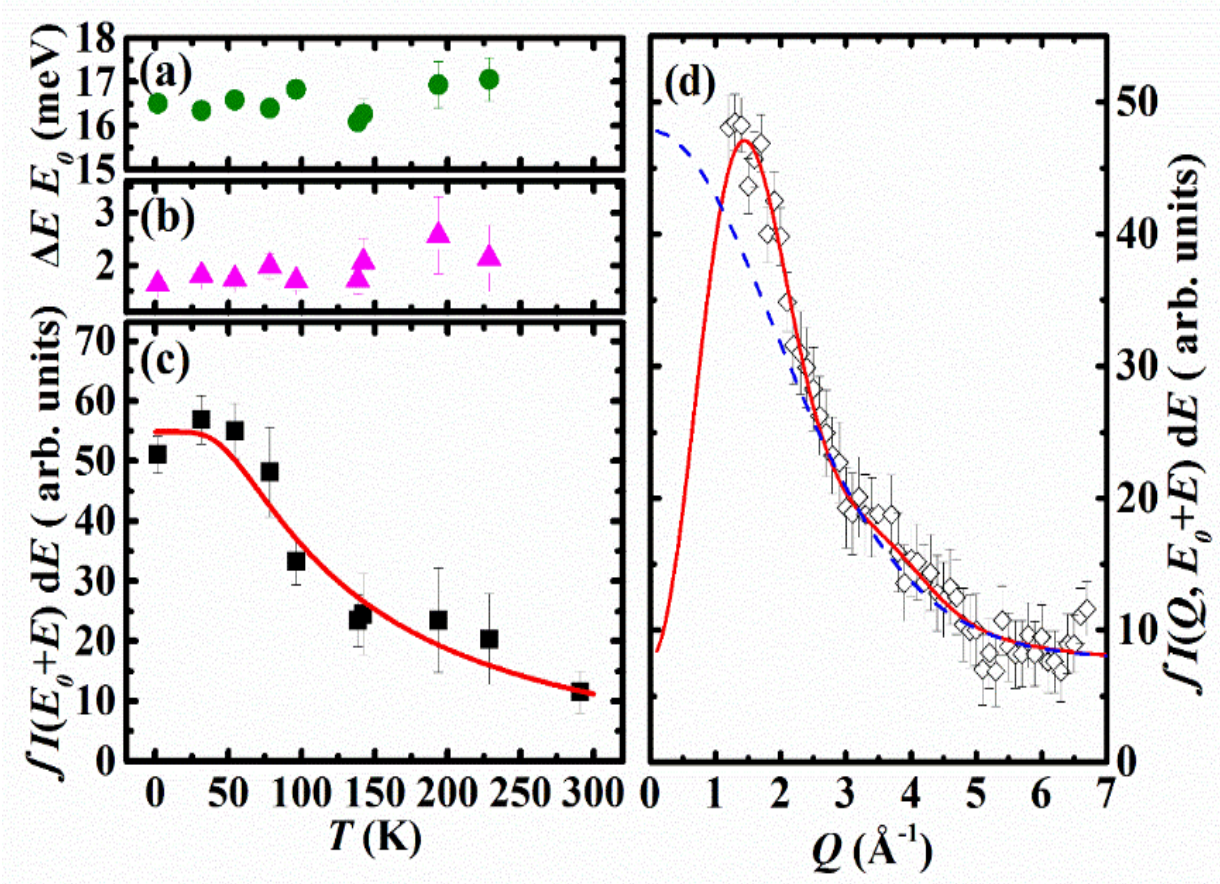

Figure 3.4 Temperature dependence of dimer excitation in $\operatorname{BCGO}(p=0.902(8))$

(a), (b) T dependence of the excitation energy and resolution corrected FWHM of the lowest energy inelastic peak in wave-vector integrated neutron scattering from $\operatorname{BCGO}(p=0.902(8))$. (c) $Q$ - and $\omega$ - integrated intensities of the $16.5 \mathrm{meV}$ excitations versus $\mathrm{T}$. The red solid line was calculated from Equation (1). (d) The $Q$ dependence of the energy integrated neutron intensity at $T=1.5 \mathrm{~K}$. In (c), the position and width of the peaks at each value of $Q$ were fixed to the average obtained in individual fits. Blue dashed line in (c) shows the magnetic form factor of $\mathrm{Cr}^{3+}$, the red solid line is the calculated Q dependence of the neutron scattering cross-section in Equation 3.1.

Similar dispersionless magnetic excitations in $\operatorname{SCGO}(p=0.925)$, can be explained by a model of $4 f_{v i}-4 f_{v i}$ spin dimers. To determine whether the model of isolated spin dimers works for $\operatorname{BCGO}(p=0.902(8))$, we perform a quantitative comparison of our data to the neutron scattering cross section for exchange-coupled pairs of $s=3 / 2 \mathrm{Cr}^{3+}$ ions in powder samples, [53,54]

$$
\begin{aligned}
\frac{d^{2} \sigma}{d \Omega d E^{\prime}}=r_{0}^{2} \frac{k^{\prime}}{k} & N z\left\{\frac{1}{2} g F(Q)\right\}^{2} \frac{1}{Z}\left(1-\frac{\sin (Q R)}{Q R}\right) \\
\times & {\left[5 \delta(\hbar \omega-J)+8 \exp \left(\frac{-J}{k_{B} T}\right) \delta(\hbar \omega-2 J)+8 \exp \left(\frac{-3 J}{k_{B} T}\right) \delta(\hbar \omega-3 J)\right] }
\end{aligned}
$$

where $R$ is the separation between spins in $z$ spin dimers per formula units and $Z=1+$ $3 \exp \left(-J / k_{B} T\right)+5 \exp \left(-3 J / k_{B} T\right)+7 \exp \left(-6 J / k_{B} T\right)$ is the partition function. The 
$\delta$-functions are for the corresponding dimmer excitations. Due to the energy limit of DCS, only the excitation from ground state to first excited state represented by $\delta(\hbar \omega-J)$ was observed in our experiments. The solid lines in Figure 3.4 (c) and Figure 3.4 (d) were calculated from Equation 3.1 by fitting only the distance between spins, $R$, and using the number of spin dimers per formula unit $\mathrm{z}=0.79(1)$ which was obtained from refinements of the neutron powder diffraction data. Both the $Q$ dependence and the $T$ dependence of the intensity are perfectly accounted for by Equation 3.1. The parameter of $R=2.75(5) \AA$ was obtained from the fitting, corresponding to the bonding distance of each spin dimer. According to the diffraction data for $\operatorname{BCGO}(p=0.902(8))$, the $4 f_{v i}-4 f_{v i}$ spin pairs are separated by a distance of $d 5=2.72(7) \AA$ (see the Figure 3.1 (a)) which is very close to the fitted parameter of $R=2.75(5) \AA$. This confirms that the $4 f_{v i} \mathrm{Cr}^{3+}$ ions form isolated spin dimers with an AFM coupling constant $J=16.5(1) \mathrm{meV}$.

\subsection{Inelastic Neutron Scattering Spectrum}

To further investigate the INS spectrum of $\operatorname{BCGO}(p=0.902(8))$, we have performed more neutron time-of-flight (TOF) experiments similar to the previous section at four different initial wavelengths, $1.5 \AA, 2.7 \AA, 4.5 \AA$ and $6 \AA$ covering almost three decades of neutron transfer energy $(\hbar \omega)$ ranging $0.1 \mathrm{meV}$ to $23 \mathrm{meV}$. All the measurement were done at $T=$ $1.5 \mathrm{~K}$ on $\operatorname{BCGO}(p=0.902(8))$ in comparison to $\operatorname{SCGO}(p=0.968(6))$ and $\operatorname{SCGO}(p=$ $0.736(6))$ samples. Similar to the previous section, the $Q$-integrated intensity $I(\omega)=$ $\int_{0.5 \AA^{-1}}^{1.8 \AA^{-1}} I(Q, \omega)\left[\frac{Q}{|F(Q)|}\right]^{2} d Q / \int_{0.5 \AA^{-1}}^{1.8 \AA^{-1}} Q^{2} d Q$ was calculated for all the three samples and the corresponding imaginary part of dynamic susceptibilities, $\chi^{\prime \prime}(\omega)$, were calculated by $\chi^{\prime \prime}(\omega)=\pi\left\{1-e^{-\frac{\hbar \omega}{k_{B} T}}\right\} I(\omega)$, where $k_{B}$ is the Boltzmann factor. (see Figure 3.5) To combine multiple datasets with different instrumental energy resolutions $\left(\Delta \omega_{0}\right)$, each individual measurement has been normalized to $\Delta \omega_{0}$ and estimated the constant background for each dataset in order to match the intensities of the overlapping regions. 


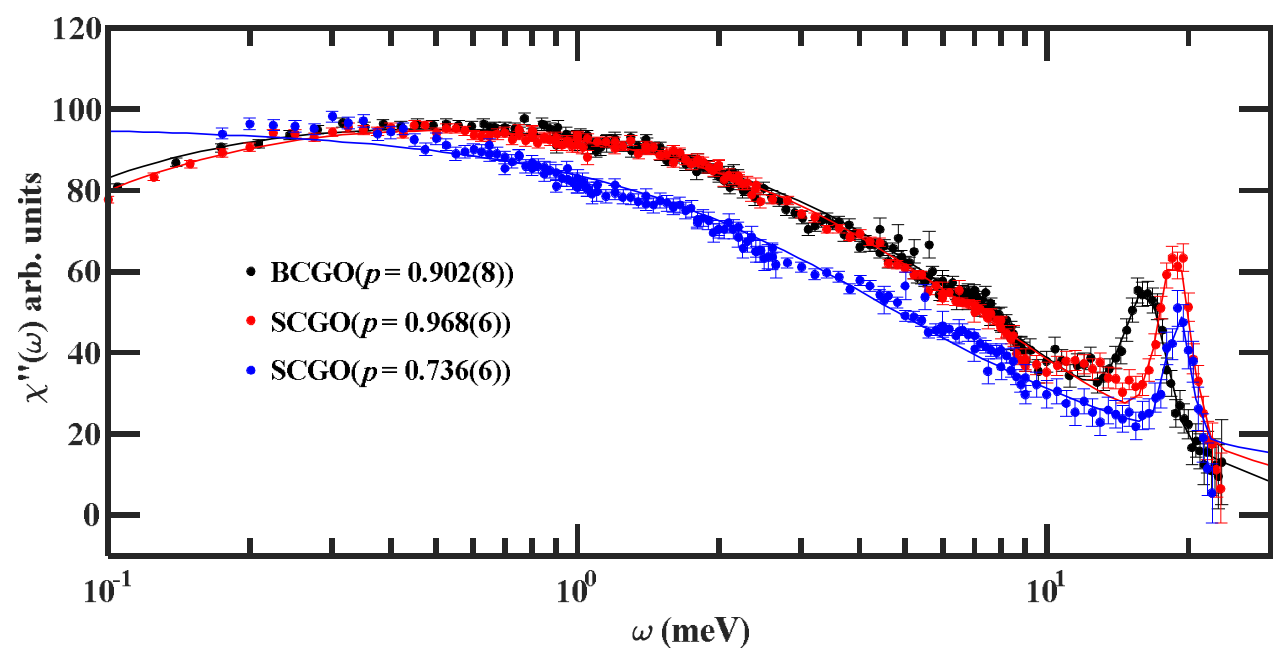

Figure 3.5 Entire neutron scattering spectrum of BCGO and SCGO systems

The imaginary part of dynamic susceptibility $\chi^{\prime \prime}(\omega)$ for $\operatorname{BCGO}(p=0.902(8)), \operatorname{SCGO}(p=$ $0.968(6))$ and $\operatorname{BCGO}(p=0.736(6))$ are calculated by combining Inelastic Neutron Scattering intensities measured at four different initial wave lengths, $1.5 \AA$, $2.7 \AA$, $4.5 \AA$ and $6 \AA$. All the measurements were done at $1.5 \mathrm{~K}$ using a standard ILL Orange Cryostat on Disk Chopper Spectrometer (DCS), NCNR, NIST. The solid lines are the fits of each $\chi^{\prime \prime}(\omega)$ to the Equation 3.1.

The broad low energy signal of $\chi^{\prime \prime}(\omega)$ implies that a distribution of characteristic spin relaxation rates, $\Gamma$, is present, which is common in glassy materials [7]. A distribution of $\Gamma$ would yield,

$$
\chi^{\prime \prime}(\omega) \propto \int_{\Gamma_{m n}}^{\Gamma_{\max }} \frac{\omega}{\omega^{2}+\Gamma^{2}} d \Gamma=\left[\tan ^{-1}\left(\frac{\omega}{\Gamma_{\min }}\right)-\tan ^{-1}\left(\frac{\omega}{\Gamma_{\max }}\right)\right]
$$

The entire inelastic scattering spectrum can be fit by the following function,

$$
\chi^{\prime \prime}(\omega)=A \times\left[\tan ^{-1}\left(\frac{\omega}{\Gamma_{1}}\right)-\tan ^{-1}\left(\frac{\omega}{\Gamma_{2}}\right)\right]+B \times e^{-\frac{\left(\omega-\omega_{0}\right)^{2}}{2 \sigma^{2}}}+C
$$

The first term is for the relaxation dynamics and the second term fits the singlet to triplet excitation of the isolated dimers and third term fits the constant background. The solid lines in Figure 3.5 show the corresponding fits and Table 3-2 summarizes the fitting parameters.

The upper bound of the distribution of spin relaxations, $\Gamma_{\max }$ can be as high as 8.1(2) meV for $\operatorname{BCGO}(p=0.902(8))$ at $1.5 \mathrm{~K}$, which is quite large comparing to the $\Gamma_{\min }=0.35(2)$ meV of the same dataset. Quantitatively, $\Gamma_{\max }$ and $\Gamma_{\min }$ of $\operatorname{BCGO}(p=0.902(8))$ and 
$\operatorname{SCGO}(p=0.968(6))$ samples are comparable. Moreover, the low-energy spectrum of $\chi^{\prime \prime}(\omega)$ shows a suppression in both material by concluding both systems have similar the spin relaxation distributions at the clean limit $(p \rightarrow 1)$. But, an energy independent $\chi^{\prime \prime}(\omega)$ is expected for conventional spin glasses such as $C u \mathrm{Mn}$. [7] Thus, this is a clear evidence that both $\operatorname{BCGO}(p=0.902(8))$ and $\operatorname{SCGO}(p=0.968(6))$ belong to a different category of magnetic glass distinct from the conventional spin glass.

Table 3-2 Fitting parameters of the inelastic spectrum of $\operatorname{BCGO}(p)$ and $\operatorname{SCGO}(p)$ samples. The dynamic susceptibility $\chi^{\prime \prime}(\omega)$ of $\operatorname{BCGO}(p=0.902(8)), \operatorname{SCGO}(p=0.968(6))$ and $\operatorname{SCGO}(p=$ $0.736(6))$ samples has been fitted to the Equation 3.1, and the parameters $\Gamma_{\max }, \Gamma_{\min }$ and $\omega_{0}$ are summarized here.

\begin{tabular}{lccc}
\hline \hline Parameter & $\mathrm{BCGO}(\mathrm{p}=0.902(8))$ & $\mathrm{SCGO}(\mathrm{p}=0.968(6))$ & $\mathrm{SCGO}(\mathrm{p}=0.736(6))$ \\
\hline $\boldsymbol{\Gamma}_{\boldsymbol{m i n}}(\mathrm{meV})$ & $0.027(3)$ & $0.035(2)$ & $2.54 \mathrm{e}-10$ \\
$\boldsymbol{\Gamma}_{\boldsymbol{m a x}}(\mathrm{meV})$ & $8.1(2)$ & $7.10(26)$ & $4.6(2)$ \\
$\boldsymbol{\omega}_{\mathbf{0}}(\mathrm{meV})$ & $16.32(5)$ & $18.82(4)$ & $19.14(2)$ \\
\hline
\end{tabular}

In contrast, the low-energy spectrum of $\operatorname{SCGO}(p=0.736(6))$ does not show a suppression, making the $\Gamma_{\min } \approx 0$ within the instrumental energy resolution of $25 \mu \mathrm{eV}$. The $\Gamma_{\max }$ is also lower than in other two samples. Thus the entire spin relaxation rate distribution in $\operatorname{SCGO}(p=0.736(6))$ has moved towards longer times. This fact hints that the glassiness in the clean limit has been evolve into a distinct glass phase over dilution, leading to further investigations as a function of dilution, as discussed in Chapter 4 .

\subsection{Quasi-Elastic Neutron Scattering}

\subsubsection{Neutron Time-of-Flight experiments}

To characterize the magnetic glass phase found in the clean limit of the quasi-2D network, we have done further analysis on the Neutron scattering data measured on $\operatorname{SCGO}(p=$ $0.968(6))$ at wavelength $6 \AA$ and temperature of $1.5 \mathrm{~K}$. The data exhibit a continuum spectrum centered in the vicinity of $Q_{\max } \approx 1.5 \AA^{-1}$ that corresponds to $(2 / 3,2 / 3,1.8)$, confirming that the kagome-triangular-kagome trilayer is responsible for the low-energy dynamic spin correlations [5]. (See Figure 3.6 (a)) This resembles the energy continuum expected for spin liquids or cooperative paramagnets; however, in contrast to a spin liquid, 
in our system, static spin correlations develop below $T_{f}$ as well. The $Q-$ dependence of the elastic magnetic neutron scattering intensity, $I_{e l}^{m a g}(Q)=I_{e l}(Q, 1.4 \mathrm{~K})-I^{e l}(Q, 20 \mathrm{~K})$, was obtained with an energy window of $|\hbar \omega| \leq 25 \mu \mathrm{eV}$, where the subtraction of the signal above the magnetic transition eliminates background from sources not related to the transition. As shown in Figure $3.6(\mathrm{~b}), I_{e l}^{m a g}(Q)$ exhibits a broad peak at $Q_{\max }=1.49 \AA^{-1}$. Its broadness indicates the short-range nature of the static spin correlations. Moreover, we fit $I_{e l}^{m a g}(Q)$ to a simple Lorentzian function, $I_{e l}^{m a g}(Q) \propto 1 /\left[\mathrm{HWHM}^{2}+\left(Q-Q_{\text {max }}\right)^{2}\right]$ where HWHM is the half width at half maximum of $I_{e l}^{m a g}(Q)$. As a measure of the static spin correlation length scale of the frozen state, we can use $\xi_{H W H M}^{\text {powder }}=1 /$ HWHM. Note that the powder averaging would introduce extrinsic broadening to $I_{e l}^{m a g}(Q)$, and thus $\xi_{\mathrm{HWHM}}^{\text {powder }}$ is underestimated, in comparison with the correlation length determined from single-crystal data, $\xi_{\mathrm{HWHM}}^{\text {crystal }}=4.6(2) \AA$ for $\operatorname{SCGO}(p \sim 0.67)[5]$.
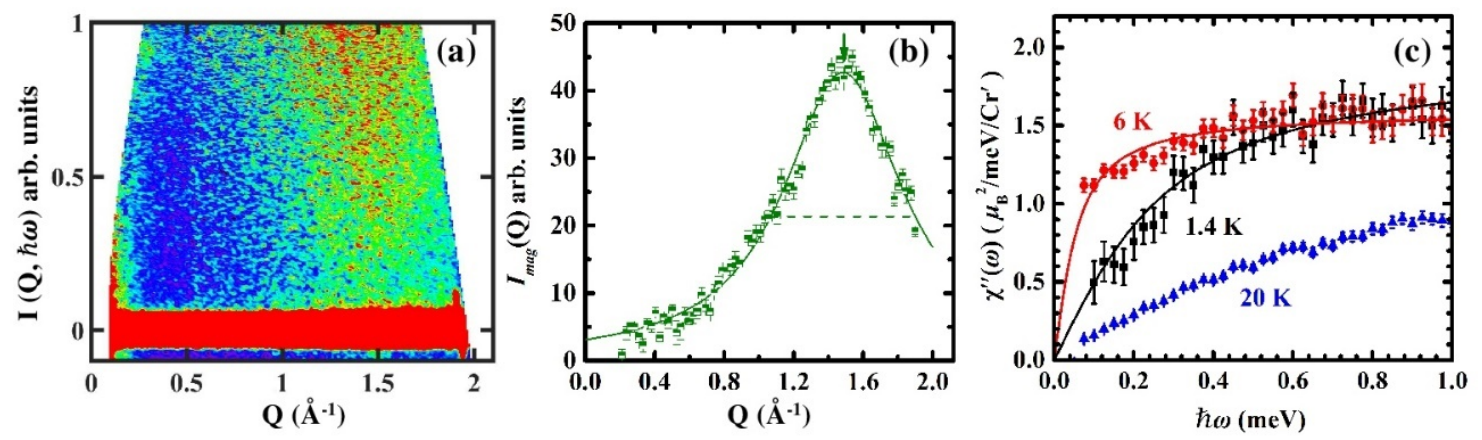

Figure 3.6 Quasi-Elastic Neutron Scattering spectrum of $\operatorname{SCGO}(p=0.968(6))$

(a) The contour map of neutron scattering intensity as a function of momentum and energy transfer, (b) Q dependence of the elastic magnetic scattering intensity and (c) The imaginary part of the dynamic susceptibility at low energies was obtained from the inelastic neutron scattering intensities measured at three different temperature for $\operatorname{SCGO}(p=0.968(6))$ are summarized here. The neutron scattering measurements were performed with incident neutron wavelength of $6 \AA$ and $T=1.4 \mathrm{~K}$. The intensities were normalized to an absolute unit by comparing them to the $(0,0,2)$ nuclear Bragg peak intensity. Nonmagnetic background at the elastic line was determined at $20 \mathrm{~K}$ while the temperature independent nonmagnetic background as a function of $\omega$ was estimated by comparing inelastic signals at the three different temperatures. The Solid line in (b) fits the $I(Q)$ to a simple Lorentzian while it is in (c), fits $\chi^{\prime \prime}(\omega)$ to an arctangent. Dashed line and arrows in (b) represents the fitted FWHM and peak position, respectively.

In order to study low-energy excitation more carefully, we present $\chi^{\prime \prime}(\omega)$ for $\operatorname{SCGO}(p=$ $0.968(6))$ as a function temperature. Upon cooling in the cooperative paramagnetic state 
from $20 \mathrm{~K}\left(\ll\left|\theta_{c w}\right|\right)$ to $5 \mathrm{~K}\left(\sim T_{f}\right), \chi^{\prime \prime}(\hbar \omega)$ changes from being linear to almost flat (see Figure 3.6 (c)). The nearly $\omega$-independent low-energy $\chi^{\prime \prime}$ implies that a distribution of the characteristic spin relaxation rates, $\Gamma$, is present, which is common in glassy transitions [7]. For more quantitative analysis, we fit the data to a simplified version of Equation 3.1, $\chi^{\prime \prime}(\omega) \propto \tan ^{-1}\left(\omega / \Gamma_{\text {min }}\right)$ where the rest of terms are ignored, since $\Gamma_{\max }(\gg 1 \mathrm{meV})$ is much larger than the $\omega$-range of interest and the dimer excitation is also not present in this energy range.

Cooling from the cooperative paramagnetic state $(\mathrm{T}=20 \mathrm{~K})$ to $\mathrm{T}=6 \mathrm{~K}\left(\sim T_{f}\right)$, the lower bound of the distribution of $\Gamma$ decreases from $\Gamma_{\min }>1 \mathrm{meV}$ to a very low value of $0.053(4)$ meV. (See the blue and red solid lines in the Figure 3.6 (c)). Upon further cooling into the frozen state, however, $\chi^{\prime \prime}(\hbar \omega)$ exhibits hardening: The weight gets depleted and becomes linear at low energies (see the $1.4 \mathrm{~K}$ data), which is consistent with a previous neutron scattering study of $\operatorname{SCGO}(p=0.92(5))$ [33]. The $1.4 \mathrm{~K}$ data can still be fitted to $\chi^{\prime \prime}(\omega) \propto$ $\tan ^{-1}\left(\omega / \Gamma_{\text {min }}\right)$ with $\Gamma_{\text {min }}=0.25(3) \mathrm{meV}$, which indicates that a distribution of $\Gamma$ is still present but with the larger minimum cutoff than that of $6 \mathrm{~K}$. Also, for $\hbar \omega \lesssim 2 \Gamma_{\min }, \chi^{\prime \prime}(\omega)$ is linear with $\omega$ implying a glass phase different from conventional spin glasses in which low energy spin dynamics shows $\omega$ - independent behavior. 


\subsubsection{Neutron Spin Echo experiments}

The lowest energy resolution can be achieved in a standard direct geometry time-flight (TOF) spectrometer is about one-tenth of a meV which is corresponding to pico-second relaxations. On the other hand, one always has to trade intensity for the energy resolution in TOF spectrometer, and it is not easy to achieve $\mu \mathrm{eV}$ energy resolutions from standard TOF spectrometers. To overcome this challenge, the Neutron Spin Echo spectroscopy was invented by Mezei in 1972 [30] in which we have access to nanosecond regime of relaxation time. For magnetic glasses, slow relaxation studies are important, especially in nanosecond timescales for proper characterization. [55--57]

In order to investigate nanosecond regime of relaxation time in spin jam state, we have performed Neutron Spin Echo measurements on $\operatorname{BCGO}(p=0.902(8))$ at Neutron Spin Echo (NSE) spectrometer, NIST with a neutron beam of wavelength $\lambda=6 \AA$ and bandwidth $\Delta \lambda / \lambda=20 \%$, covering the dynamic range of $3 \mathrm{ps}$ to $10 \mathrm{~ns}$. For the measurements in the range of Fourier time $(t), 0.05 \mathrm{~ns} \leq t \leq 10 \mathrm{~ns}$, a single coil setting was used for NSE electromagnets while a two-concentric coil setting in which the two concentric coils operates in opposite fashion to produce a small but stable effective magnetic field at the center, was used to access the faster dynamics in the range of $3 \mathrm{ps} \leq$ $t \leq 0.01 \mathrm{~ns}$.

In the NSE spectroscopy, we measure intermediate scattering function, $I(Q, t)$ in reciprocal space and real time which is the temporal Fourier Transformation of usual scattering function $S(Q, \omega)$. Moreover, we can get rid of instrument resolutions from a measurement just by dividing it by the resolution function measured on a magnetic standard sample as an advantage of working in Fourier space of $S(Q, \omega)$. Here we estimated instrument resolution by measuring a $\mathrm{Ho}_{2} \mathrm{Ti}_{2} \mathrm{O}_{7}$ sample at $T=1.5 \mathrm{~K}$. $\mathrm{BCGO}(p=0.902(8))$ was first measured for $I(Q, t=5 \mathrm{~ns})$ as a function of the wavevector $(Q)$ in the $0.1 \AA^{-1} \leq Q \leq$ $1.5 \AA^{-1}$ range and observed a broad peak centered around $Q \approx 1.5 \AA^{-1}$ similar to the previous Neutron TOF measurement shown in Figure 3.6 (a) and decided to fix $Q$ at $1.5 \AA^{-1}$ where the maximum signal observed. 

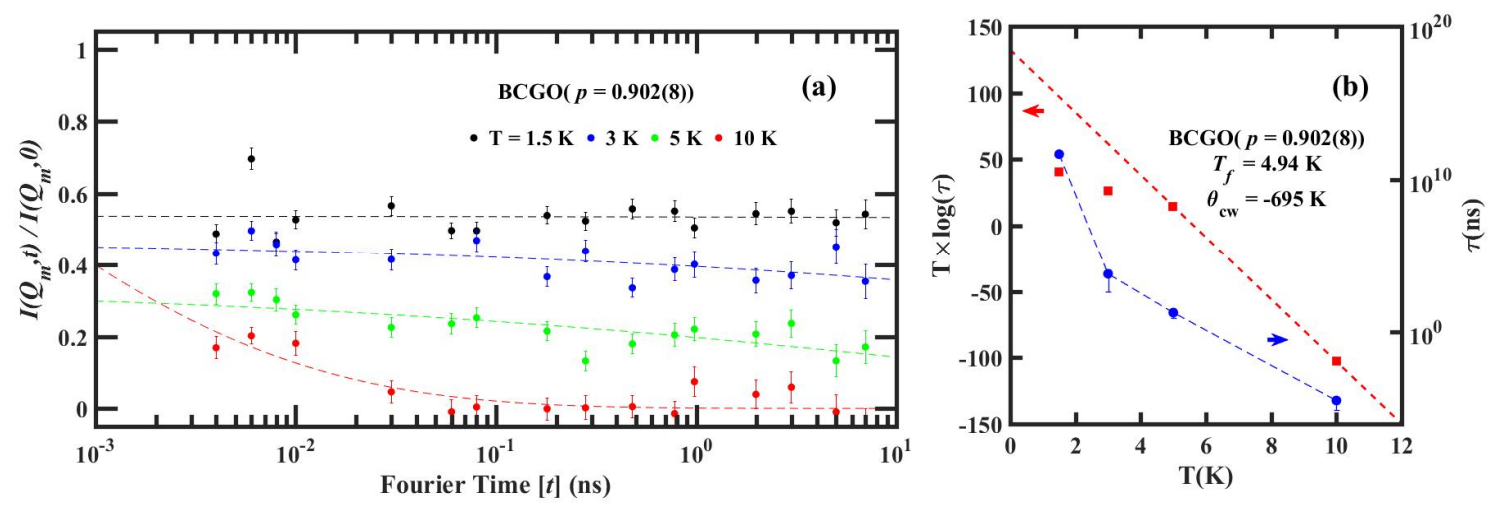

Figure 3.7 Neutron spin echo spectroscopy of $\operatorname{BCGO}(p=0.902(8))$

(a) The normalized intermediate scattering function, $I\left(Q_{m}, t\right) / I\left(Q_{m}, 0\right)$, where $Q_{m}$ was fixed at $1.5 \AA^{-1}$, of $\operatorname{BCGO}(p=0.902(8))$ was measured as a function of Fourier time, $t$, at four different temperatures of $1.5 \mathrm{~K}, 3 \mathrm{~K}, 5 \mathrm{~K}$ and $10 \mathrm{~K}$. The measurements were taken using the NSE spectrometer at NIST in the Fourier time range of $3 \mathrm{ps} \leq t \leq 10 \mathrm{~ns}$. All the $I\left(Q_{m}, t\right) / I\left(Q_{m}, 0\right)$ were fitted to the function $I\left(Q_{m}, t\right) / I\left(Q_{m}, 0\right)=A * \exp \left[-t^{0.2} / \tau\right]$ where $\tau$ is the relaxation time. (b) summarizes the estimated $\tau$ (blue) and $T \times \log (\tau)$ (red) as a function of temperature $(T)$. The red dash line is a linear fit to the $T \times \log (\tau)$ data above the magnetic transition temperature $T_{D C} \sim$ $4.9 \mathrm{~K}$ while the blue dash line just connects data points.

Figure 3.7 (a) shows $I(Q, t, T)$ measured on $\operatorname{BCGO}(p=0.902(8))$, as a function of Fourier time, $t$ at a fixed $Q=1.5 \AA^{-1}$ and four temperature $1.5 \mathrm{~K}, 3 \mathrm{~K}, 5 \mathrm{~K}$ and $10 \mathrm{~K}$. All the measurements have been normalized by the total magnetic scattering $I(Q, t=0, T)$ calculated from the six polarization measurements done after each neutron echo scans. During the polarization measurements the electromagnets of the spectrometer was turned off and the neutron polarization along three orthogonal directions (X,Y and $\mathrm{Z}$ ) were selected by the XYZ orthogonal coil set located at the sample space. $I(Q, t=0, T)$ was determined by a subsequent polarization analysis as described in Appendix E. The $I(Q, t) / I(Q, t=0)$ can be interpreted as the static/frozen moment of the system below a certain relaxation time $t$. Note that all the relaxation slower than $t$ will be accounted as static in elastic Neutron Scattering spectroscopy. Thus, as shown in the Figure 3.7 (a), only $60 \%$ of magnetic moment are frozen at $1.5 \mathrm{~K}$. From inelastic neutron scattering measurements, we know that there is singlet to triplet excitations at $16.1 \mathrm{meV}$ which also shows maximum at $Q \sim 1.5 \AA^{-1}$. Therefore, it might be reasonable to normalize $I(Q, t) / I(Q, t=0)$ by a factor of $7 / 9$ which is the ratio of $\mathrm{Cr}^{3+}$ ions reside in the trilayer to the total $\mathrm{Cr}^{3+}$ ions, yet we can recover only up to $80 \%$ of total moment. The missing 
magnetic moment might be responsible for quantum fluctuation and the thermal fluctuations at $1.5 \mathrm{~K}$. Unlike the conventional spin glass $C u \mathrm{Mn}$, this magnet does not show an exponential decay in the nanosecond regime instead a nearly constant trend implying the relaxation time distribution is quite different from spin glasses. [56,57]

For a detailed analysis, we have fitted $I(Q . t) / I(Q, t=0)$ at each $T$ using a stretched exponential line-shape, $s(t)=s_{i} \exp \left(-(t / \tau)^{\beta}\right)$ which describes well the observed slow decay, with a fixed value $\beta=0.2$. [39] Here $\tau$ is the characteristic relaxation time and summarized in Figure 3.7 (b) (see blue markers) as a function of $T$. Apparently, $\tau(T)$ above $T_{D C} \sim 4.9 \mathrm{~K}$ follows Arrhenius law, $\tau=\tau_{0} \exp \left[\frac{E_{a}}{k_{B} T}\right]$, where $E_{a}$ is the thermal activation energy and $\tau_{0}$ is the characteristic time for Korringa relaxation at high temperature. As shown in Figure $3.7(\mathrm{~b})$, we fit $T \times \log [\tau(T)]$ as a function of $T$ above $T_{D C} \sim 4.9 \mathrm{~K}$ to a straight line in which the intercept directly measures $E_{a} / k_{B}$. The estimated $E_{a} / k_{B} \sim 132$ $\mathrm{K}$ and it is consistent with energy scale defined by $\left|\theta_{c w}\right|$. Similar thermally activated nature in the energy scale of $\left|\theta_{c w}\right|$ which represents the averaged interaction energy of the system, can be usually expected in magnetic phases with correlated dynamics such as spin liquids. Thus, it confirms that the magnetic phase at the proximity to the low temperature magnetic transition defined by $T_{D C}$ in $\mathrm{BCGO}(p=0.902(8))$ is liquid like or cooperative paramagnetic and the deviation from Arrhenius law below $T_{D C}$ is an indication of magnetic transition to a different magnetic phase in which relaxation time distribution is different from conventional spin glasses. 


\subsection{Discussion and Conclusions}

In summary, we synthesized a new frustrated chromate $\operatorname{BCGO}(p)$ over a wide range of the Cr concentration $0.4 \lesssim p \lesssim 0.9$. Our neutron powder diffraction data show that $\operatorname{BCGO}(p)$ is isostructural to $\operatorname{SCGO}(p)$ but with a much larger c lattice constant. Our bulk magnetic susceptibility data show that $\operatorname{BCGO}(p)$ exhibits similar glassy behavior as $\operatorname{SCGO}(p)$ at low temperatures with a higher freezing temperature $T_{f}$ than $\operatorname{SCGO}(p)$. The frustration index $\left|\theta_{c w}\right| / T_{f}$ is as high as 135 for $\operatorname{BCGO}(p=0.902(8))$ indicating strong frustration. Similar to $\operatorname{SCGO}(p=0.925)$, inelastic neutron scattering shows that $\operatorname{BCGO}(p=0.902(8))$ has a dispersionless magnetic excitation centered at $\hbar \omega=16.5(1) \mathrm{meV}$, which is due to the singlet to triplet excitation of spin dimers formed by two $s=3 / 2$ spins of two neighboring $4 f_{v i}$ layers that exist in between $12 k-2 a-12 k$ trilayers. The formation of the spin dimers in the $4 f_{v i}$ layers effectively makes the $12 k-2 a-12 k$ trilayers excellent quasi2D magnetic lattices. This also tells us that the kagome-triangular-kagome $(12 k-2 a-$ $12 \mathrm{k}$ ) trilayers or the triangular network of bipyramids are responsible for the freezing behavior at low temperatures observed in bulk magnetic susceptibility (see Figure 3.2 (b)) and the low energy continuum behavior in inelastic neutron scattering (see Figure 3.6 (a)).

Moreover, we have performed neutron time-of-flight experiments with several different neutron wavelengths and observed the inelastic neutron scattering spectrum over a broad range of excitation energy, and the imaginary part of magnetic susceptibility can be modeled with a simple bounded distribution of relaxation time in which upper bound can be high as $8 \mathrm{meV}$ at the transition temperature $\left(T_{D C}\right)$ while lower bound can be as low as 0.053(4) meV. Over further cooling, we observed a suppression of intensity at low energies by indicating a magnetic glass phase distinct form spin glasses [7] as confirmed by Neutron Spin Echo experiments. In the Neutron Spin Echo experiments, we observed thermally activated behavior above $T_{D C}$ concluding the low temperature magnetic transition is from liquid like phase to an exotic magnetic glass phase (spin jam). 


\section{Chapter 4}

\section{Crossover from Spin Jam to Spin Glass upon dilution in $\operatorname{SCGO}(p)$}

\subsection{Motivation}

In the previous chapter, we discussed quasi-two-dimensional antiferromagnetic systems $\operatorname{SCGO}(p) / \operatorname{BCGO}(p)$ which shows magnetic glass-like freezing at low temperatures along with unconventional spin glass properties. Defects and frustration have been discussed as the freezing mechanism of this material similarly in canonical dilute spin glasses. [58] Recently, an alternative freezing mechanism in which a spin jam state is induced by quantum fluctuations at low temperature, was proposed for a disorder-free quasi-2D ideal SCGO lattice with a simple nearest neighbor (NN) spin interaction Hamiltonian, $H=$ $J \sum_{N N} S_{i} . S_{j}[4,5]$. The spin jam framework provided a qualitatively coherent understanding of all of the low-temperature behaviors such as that a complex energy landscape is responsible for the frozen state without long-range order [5], and Halperin-Saslow (HS)like modes for the $C_{v} \propto T^{2}$ and $\chi^{\prime \prime}(\omega) \propto \omega$ behaviors [5,15]. In this system, which we refer to as the ideal SCGO model (iSCGO), semiclassical magnetic moments (or spins) are arranged in a triangular network of bipyramids and interact uniformly with their NN $[4,5]$. The microscopic mechanism for the spin jam state is purely quantum mechanical. The system has a continuous and flat manifold of ground states at the mean field level, including locally collinear, coplanar, and noncoplanar spin arrangements. Quantum fluctuations lift the classical ground state degeneracy (order by fluctuations), resulting in a complex rugged energy landscape that has a plethora of local minima consisting of the locally collinear states separated from each other by potential barriers [5]. Although the work of ref. [5] 
dealt with a similar phase space constriction by quantum fluctuations as the aforementioned other theoretical works did, we would like to stress here the difference between the two: whereas the other works mainly focused on the selection of the long-range ordered (LRO) energetic ground state, the work of ref. [5] showed that the short-range-ordered (SRO) states that exist at higher energies are long-lived, dominate entropically over the LRO states, and govern the low-T physics.

The introduction of non-magnetic impurities into a topological spin jam state will break some of the constraints in the system, and may possibly allow local transitions between minima, with a time scale dependent on the density of impurities. And at a sufficiently high vacancy concentration, the system will exit the spin jam state and will become either paramagnetic or an ordinary spin glass at lower temperatures. Here we try to identify and explore the spin jam regime in an experimentally accessible system. The three most important signatures we seek for the existence of a spin jam state different from conventional spin glass states and spin ice are $(i)$ linear dependence of the imaginary part of the dynamic susceptibility at low energies, $\chi^{\prime \prime}(\omega) \propto \omega$, (ii) intrinsic short-range static spin correlations, and (iii) insensitivity of its physics to nonmagnetic doping in the clean limit. In this chapter, we provide experimental demonstration of these properties.

\subsection{Experiments as a function of dilution $(p)$}

We have performed elastic and inelastic neutron scattering that directly probe spin-spin correlations and bulk susceptibility measurements on $\operatorname{SCGO}(p)$ with various values of $p$ over $0.2 \lesssim p \lesssim 1.0$ spanning almost the entire region of $p$. To first characterize the samples and to construct the $T-p$ phase diagram, we have performed dc magnetic susceptibility, $\chi_{\text {bulk }}$, and elastic neutron measurements.

The data obtained from the samples with $p=0.968(6), 0.917(9), 0.844(8), 0.777(6)$, $0.620(8), 0.459(5)$ and $0.228(5)$ are shown in Figure 4.3. For all the samples except $p=$ 0.228(5), $\chi_{\text {bulk }}$ exhibits similar field-cooled and zero-field-cooled hysteresis below $T_{f}$ ranging from $3.68 \mathrm{~K}(p=0.968(6))$ to $1.06 \mathrm{~K}(p=0.459(5))$ that are much lower than their large Curie-Weiss temperatures $\left|\Theta_{C W}\right|$ ranging from $504 \mathrm{~K}(p=0.968(6))$ to $161 \mathrm{~K}(p=$ 
0.459(5)) (see Figure 4.2). The high frustration index $f=\frac{\left|\Theta_{C W}\right|}{T_{f}} \gtrsim 130$ indicates the strong presence of frustration in all the systems. Elastic neutron scattering intensity with an instrumental energy resolution of $|\hbar \omega| \leq 25 \mu \mathrm{eV}$ starts developing at temperatures higher than their $T_{f}$ determined by $\chi_{\text {bulk }}$, as is expected for spin freezing for measurements with different energy resolution [59] . Figure 4.1 summarizes the results obtained from all the samples studied.

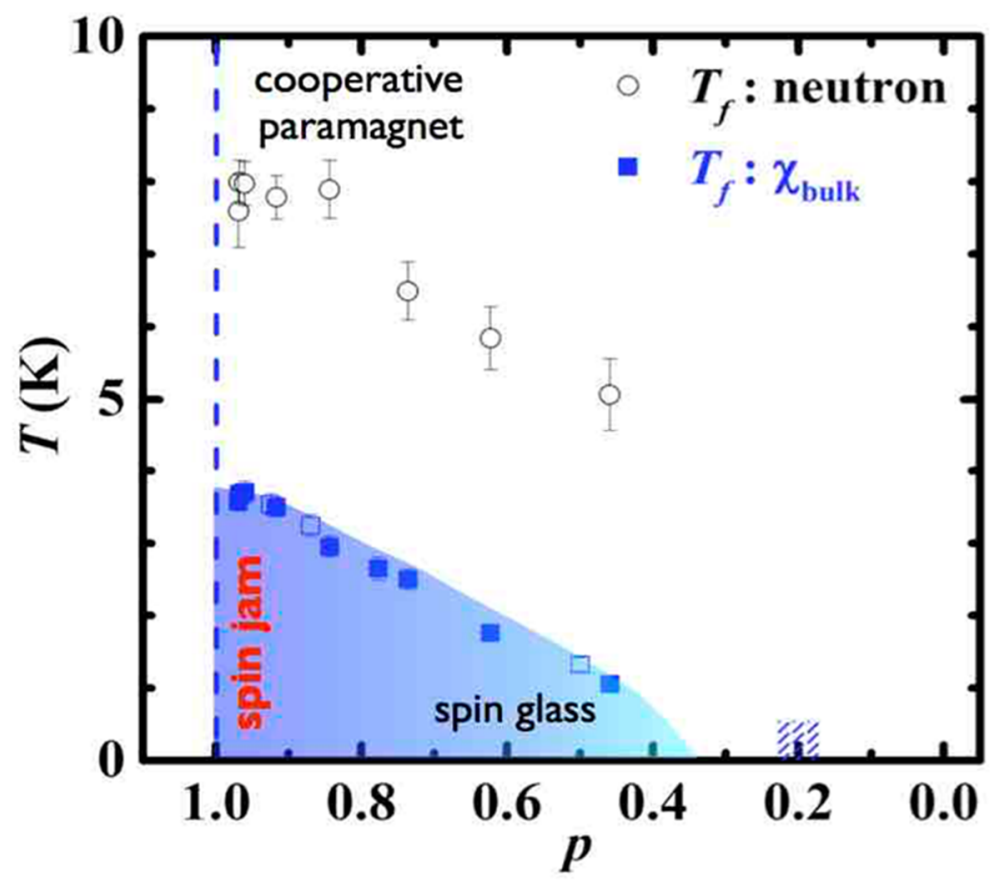

Figure 4.1 The $p$-T phase diagram of $\operatorname{SCGO}(p)$

The $p-T$ phase diagram of $\operatorname{SCGO}(p)$ constructed by bulk susceptibility and elastic neutron scattering measurements on powder samples with various $p$ values. The freezing temperatures, $T_{f}$, marked with blue square and black circle symbols are obtained by bulk susceptibility and elastic neutron scattering measurements, respectively. Filled blue squares represent the data obtained from samples whose crystal structural parameters including the $\mathrm{Cr} / \mathrm{Ga}$ concentrations were refined by neutron diffraction measurements (see Figure (App.) A.2 and Table (App.) A-1 to Table (App.) A-4), and open blue squares represent samples with nominal $p$ values. For nominal $p=0.2$, no freezing was observed down to $50 \mathrm{mK}$ (see Figure 4.3). 


\subsubsection{High-Temperature behavior as a function of $p$}

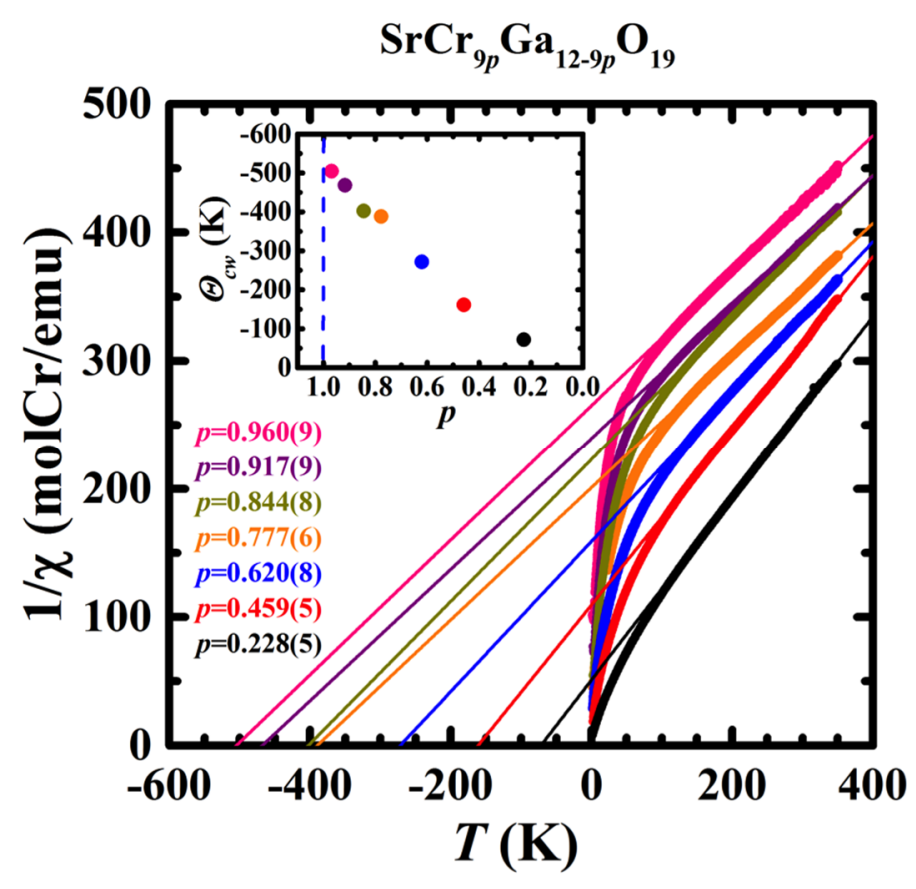

Figure 4.2 Reciprocal bulk magnetic susceptibility of SCGO (p).

Reciprocal high-Temperatre DC susceptibility of $\operatorname{SCGO}(p)$, where $\mathrm{p}$ is the range of $0.228(5) \leq p$ $\leq 0.960(9)$. The straight lines are the linear fitting at $\mathrm{T}>150 \mathrm{~K}$. The inset shows the $\Theta_{C W}$ values obtained by Curie-Weiss fitting.

Figure 4.2 shows the reciprocal susceptibility as a function of temperature ( $1 / \chi$ vs $T)$ measured in a 0.1 Tesla field over a temperature range of $2-300 \mathrm{~K}$. The Curie-Weiss temperatures $\left(\Theta_{C W}\right)$ were extracted by fitting the $1 / \chi-T$ curves at $T>150 \mathrm{~K}$ with CurieWeiss law $\left(\chi=C /\left(T-\Theta_{C W}\right)\right)$. The $\Theta_{C W}$ values range from -72 to $-504 \mathrm{~K}$, reflecting the strong anti-ferromagnetic interactions in the system. As shown in the Figure 4.2 inset, $\left|\Theta_{C W}\right|$ decreases gradually with decreasing Cr concentration from $p=0.960(9)$ to $0.228(5)$. (see Table (App.) A-9) 


\subsubsection{Low-temperature magnetic susceptibility and elastic neutron intensity.}
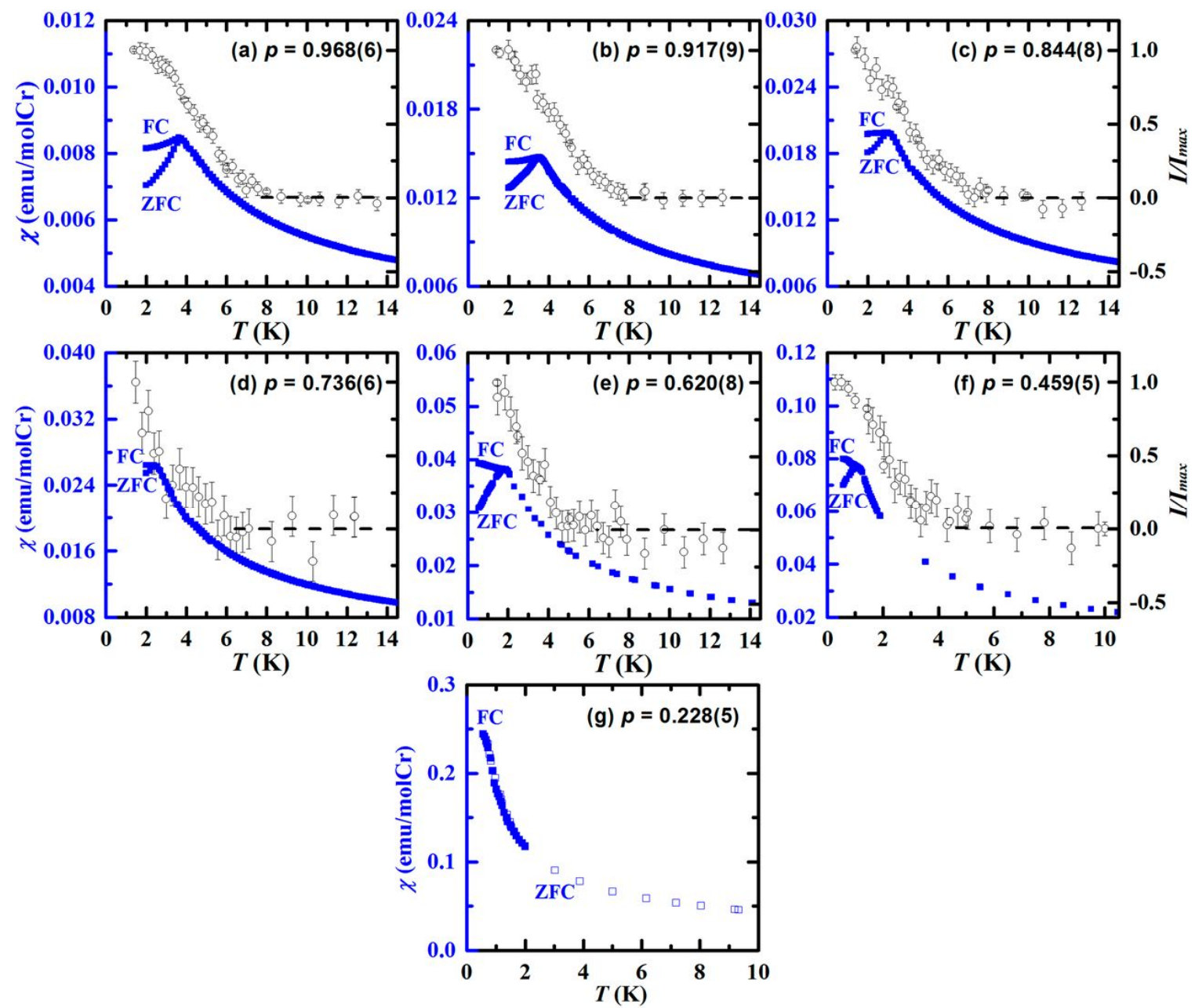

Figure 4.3 Order parameters of $\operatorname{SCGO}(p)$.

Temperature-dependent magnetic susceptibility (blue solid squares) in $\mathrm{H}=0.01 \mathrm{~T}$ and the wave vector averaged magnetic elastic neutron scattering intensity (black open circles) for SCGO [0.459(5) $\leq p \leq 0.968(6)]$ samples: (a) $p=0.968(6)$, (b) $p=0.917(9)$, (c) $p=0.844$ (8), (d) $p=$ $0.736(6)$, (e) $p=0.620(8)$, (f) $p=0.459(5)$, and (g) $p=0.228(5)$. The black dash lines indicate the background.

The low-temperature magnetic susceptibilities were measured in a field of 0.01 Tesla in the temperature range from $0.5 \mathrm{~K}$ to $14 \mathrm{~K}$, as shown in Figure 4.3 (blue squares). The susceptibility increases with decreasing temperature, exhibiting a low-temperature upturn. With further decreasing temperature, a divergence between the field-cooled (FC) and zero- 
field-cooled (ZFC) susceptibility, which is the typical behavior of spin-glass transition, was observed for all the six samples. The freezing temperature $T_{f}$ was determined as the temperature of the maximum susceptibility for the ZFC curve, where the divergence between FC and ZFC susceptibility also occurs. $T_{f}$ decreases with decreasing $\mathrm{Cr}$ concentration. Above $T f$, no difference was observed between FC and ZFC susceptibilities for all the samples.

\subsubsection{Quasi-Elastic Neutron Scattering}

The wave vector averaged magnetic elastic neutron scattering intensities normalized to the maximum intensity as a function of temperature are also shown in Figure 4.3(open circles). Neutron scattering data were obtained using the cold-neutron DCS at the NCNR with $\lambda=$ $6.0 \AA$. The temperature of the onset of magnetic neutron intensity is higher than the freezing temperature observed in bulk magnetic susceptibility data and decreases with decreasing Cr concentrations exhibiting a similar behavior to the one observed in the susceptibility data.

We observe strikingly different behaviors in the low energy spin dynamics of the $p=$ $0.968(6)$ and 0.459(5) despite the similar temperature dependences of $\chi_{\text {bulk }}$ and of elastic neutron scattering intensity. As shown in Figure 4.4 (a), in the frozen state of $p=0.968(6)$, the inelastic neutron scattering intensity $I(Q, \hbar \omega)$ is weak at very low energies below 0.25 $\mathrm{meV}$ and gets stronger as $\hbar \omega$ increases. In contrast, in the frozen state of $p=0.459(5)$, $I(Q, \hbar \omega)$ is strong at very low energies below $0.25 \mathrm{meV}$ and gets weaker as $\hbar \omega$ increases (see Figure 4.4 (f)). This stark difference hints that the frozen states of $p=0.968(6)$ and $0.459(5)$ are different in nature. The change of $I(Q, \hbar \omega)$ at very low energies below 0.25 $\mathrm{meV}$ is gradual with decreasing $p$ and manifest when $p=0.777(6)$, indicating that a transition to a different frozen state occurs between $p=0.776(6)$ and $0.844(8)$. 


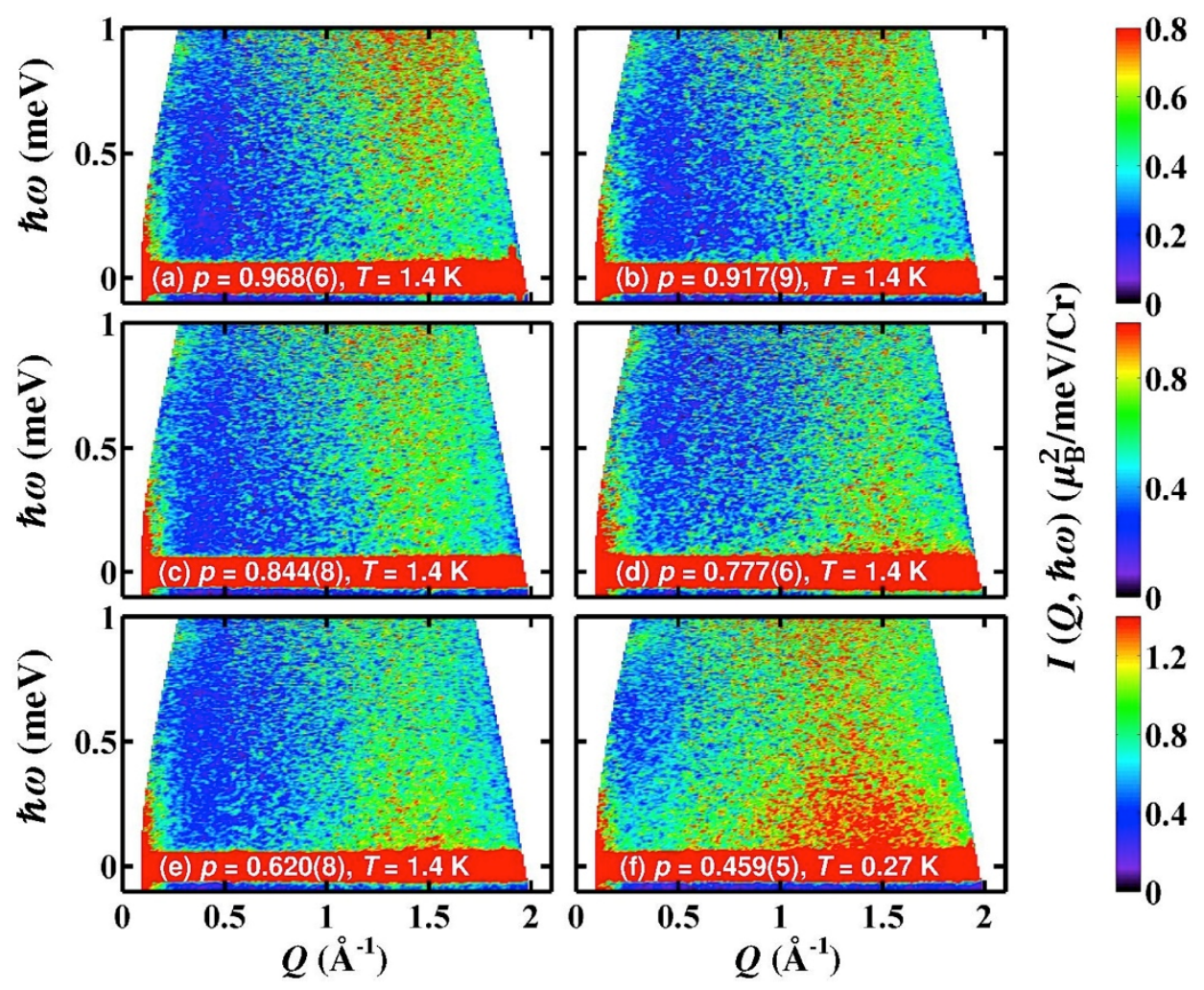

Figure 4.4 Contour maps of neutron scattering intensity $I(Q, \omega)$

Contour maps of neutron scattering intensity $I(Q, \omega)$ as a function of energy transfer $\hbar \omega$ and momentum transfer $Q$ for (a) $p=0.968(6)$ at $\mathrm{T}=1.4 \mathrm{~K}$, (b) $p=0.917(9)$ at $\mathrm{T}=1.4 \mathrm{~K}$, (c) $p=$ $0.844(8)$ at $\mathrm{T}=1.4 \mathrm{~K}$, (d) $p=0.777(6)$ at $\mathrm{T}=1.4 \mathrm{~K}$, (e) $p=0.620(8)$ at $\mathrm{T}=1.4 \mathrm{~K}$ and (f) $p=$ $0.459(5)$ at $\mathrm{T}=0.27 \mathrm{~K}$ with $\lambda=6 \AA$. Intensities were normalized to an absolute unit by scale factors obtained from the $(0,0,2)$ nuclear Bragg peak intensity.

Moreover, the data exhibits a continuum spectrum centered in the vicinity of $Q_{\max } \approx$ $1.5 \AA^{-1}$ that corresponds to $(2 / 3,2 / 3,1.8)$, confirming that the kagome-triangular-kagome trilayer is responsible for the low energy dynamic spin correlations. This resembles the energy continuum expected for spin liquids; however, in contrast to a spin liquid, in our system static spin correlations develop below $T_{f}$ as well. The $Q$-dependence of the elastic magnetic neutron scattering intensity, $I_{e l}^{m a g}(Q)=I_{e l}(Q, 1.4 K)-I_{e l}(Q, 20 K)$, was obtained with an energy window of $|\hbar \omega| \leq 25 \mu \mathrm{eV}$, where the subtraction of the signal above the magnetic transition eliminates background from sources not related to the transition. As shown in Figure 4.5, for $p=0.968(6)$, very close to the clean $\operatorname{limit}, I_{e l}^{m a g}(Q)$ exhibits a broad peak at $Q_{\max }=1.49 \AA^{-1}$. Its broadness indicates the short-range nature of the static spin correlations. Surprisingly, upon increasing nonmagnetic impurity 
concentration up to about $20 \%(p \sim 0.8)$, the shape of $I_{e l}^{m a g}(Q)$ remains the same. Only upon further doping, $I_{e l}^{\text {mag }}(Q)$ becomes broader, and the peak position $Q_{\max }(p)$ starts shifting down.
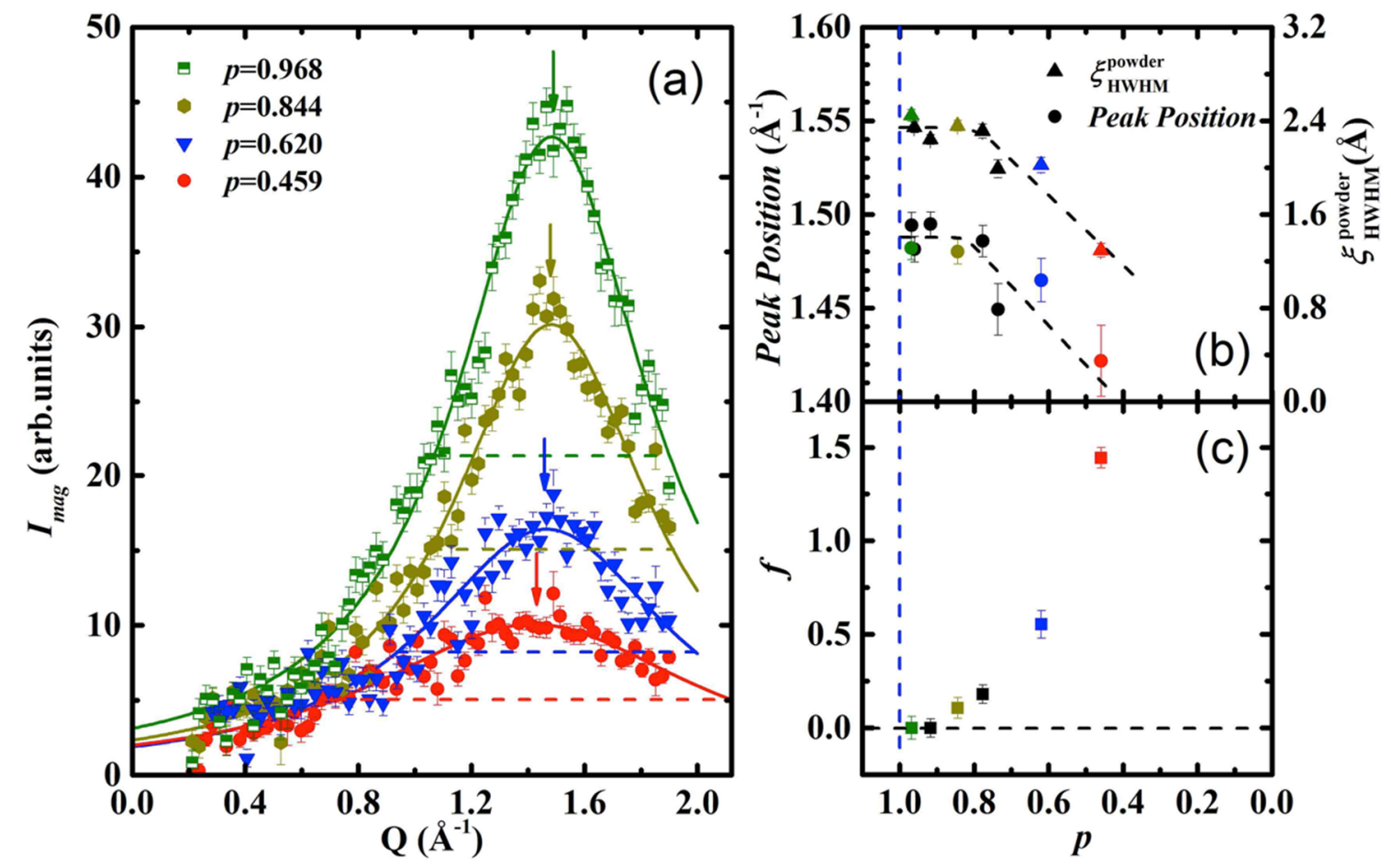

Figure 4.5 Neutron Scattering study on $\operatorname{SCGO}(p)$

(a) Q-dependence of the elastic magnetic scattering intensity measured for various values of $p$ at $1.4 \mathrm{~K}$, except for $p=0.459(5)$ at $\mathrm{T}=0.27 \mathrm{~K}$. Nonmagnetic background was determined at $20 \mathrm{~K}$ and subtracted. Solid lines are fits to a simple Lorentzian. Dashed lines and arrows represent the fitted Full-Width-of-Half-Maximum (FWHM) and peak positions, respectively. (b) The peak position and the power static spin correlation length, $\xi_{H W H M}^{\text {powder }}$, that were obtained from the fits, are plotted as a function of p. (c) The fraction, $f$, of the contribution from the single Lorentzian to the dynamic susceptibility $\chi^{\prime \prime}(\hbar \omega)$, as shown in Fig. 4, is plotted as a function of $\mathrm{p}$.

To quantitatively investigate the correlation length $\xi(p)$ and peak position $Q_{\max }(p)$ as a function of $p$, we fit $I_{e l}^{m a g}(Q)$ to a simple Lorentzian function for each $p, I_{e l}^{m a g}(Q) \propto$ $\frac{1}{H W H M^{2}+\left(Q-Q_{\max }\right)^{2}}$ where HWHM is the half-width-half-maximum of $I_{e l}^{m a g}(Q)$. As a measure of the static spin correlation length scale of the frozen state, we can use $\xi_{H W H M}^{\text {powder }}=$ $\frac{1}{H W H M}$. Note that the powder averaging would introduce extrinsic broadening to $I_{e l}^{\text {mag }}(Q)$, and thus $\xi_{H W H M}^{\text {powder }}$ is underestimated, in comparison to the correlation length determined 
from single crystal data, $\xi_{H W H M}^{\text {crystal }}=4.6(2) \AA$ for $\operatorname{SCGO}(p \sim 0.67)$ [4]. But the $p$ dependence of $\xi_{H W H M}^{\text {powder }}$ serves our search for an intrinsic state.

The resulting $\xi_{H W H M}^{\text {powder }}$ and $Q_{\max }$ are shown in Figure 4.5 (b). Remarkably, both $\xi_{H W H M}^{\text {powder }}$ and $Q_{\max }$ exhibit a flat behavior near the clean limit up to $20 \%$ doping, which is a direct evidence for the existence of a distinct phase over $1<p<0.8$ where the intrinsic shortrange static correlations are independent of nonmagnetic doping. This can be naturally described as a spin jam state at the clean limit. A spin jam state can be intrinsically short range, and has an intrinsic static correlation length, $\xi_{\text {int }}$. Thus, when nonmagnetic doping is low and the typical distance between the nonmagnetic impurities is larger than $\xi_{\text {int }}$ the spin correlations do not get affected. Only upon significant doping, the spin correlations would get disturbed to make $\xi(p)$ shorter than $\xi_{\text {int }}$ when typical distance between impurities is short.

If the $p>0.8$ phase is distinct from the lower $p<0.8$ phase, then the low energy spin excitations in those two phases should have different characteristics. To see this, we show in Figure 4.6 the imaginary part of the dynamic susceptibility $\chi^{\prime \prime}(\hbar \omega)$. For $p=0.968(6)$, upon cooling in the cooperative paramagnetic state from $20 \mathrm{~K}\left(\ll\left|\Theta_{C W}\right|\right)$ to $5 \mathrm{~K}\left(\sim T_{f}\right)$ $\chi^{\prime \prime}(\hbar \omega)$ changes from being linear to almost flat [see Figure 4.6 (a)]. The nearly $\omega$ independent low energy $\chi^{\prime \prime}$ implies that a distribution of the characteristic spin relaxation rates, $\Gamma$, is present, which is common in glassy transitions. (1) A distribution of $\Gamma$ would yield $\chi^{\prime \prime}(\omega) \propto \int_{\Gamma_{\min }}^{\Gamma_{\max }} \frac{\omega}{\omega^{2}+\Gamma^{2}} d \Gamma=\tan ^{-1} \frac{\omega}{\Gamma_{\min }}$ where a term, $\tan ^{-1} \frac{\omega}{\Gamma_{\max }}$, is ignored since $\Gamma_{\text {max }} \gg 1 \mathrm{meV}$, much larger than the $\omega$-range of interest. Fitting the $T=6 \mathrm{~K} \backsim T_{f}$ data to the model yields a distribution of $\Gamma$ with $\Gamma_{\min }=0.053(4) \mathrm{meV}$ [see the red solid line in Figure 4.6 (a)].

Upon further cooling into the frozen state, however, $\chi^{\prime \prime}(\hbar \omega)$ exhibits hardening: the weight gets depleted and becomes linear at low energies (see the $1.4 \mathrm{~K}$ data), which is consistent with a previous neutron scattering study of $\operatorname{SCGO}(p=0.92(5))$ [33]. The $1.4 \mathrm{~K}$ data can still be fitted to $\chi^{\prime \prime}(\omega) \propto \tan ^{-1} \frac{\omega}{\Gamma_{\min }}$ with $\Gamma_{\min }=0.25(3) \mathrm{meV}$, which indicates 
that a distribution of $\Gamma$, is still present, but with the larger minimum cutoff than that of $6 \mathrm{~K}$. And for $\hbar \omega \lesssim 2 \Gamma_{\min }, \chi^{\prime \prime}(\omega)$ is linear with $\omega$.

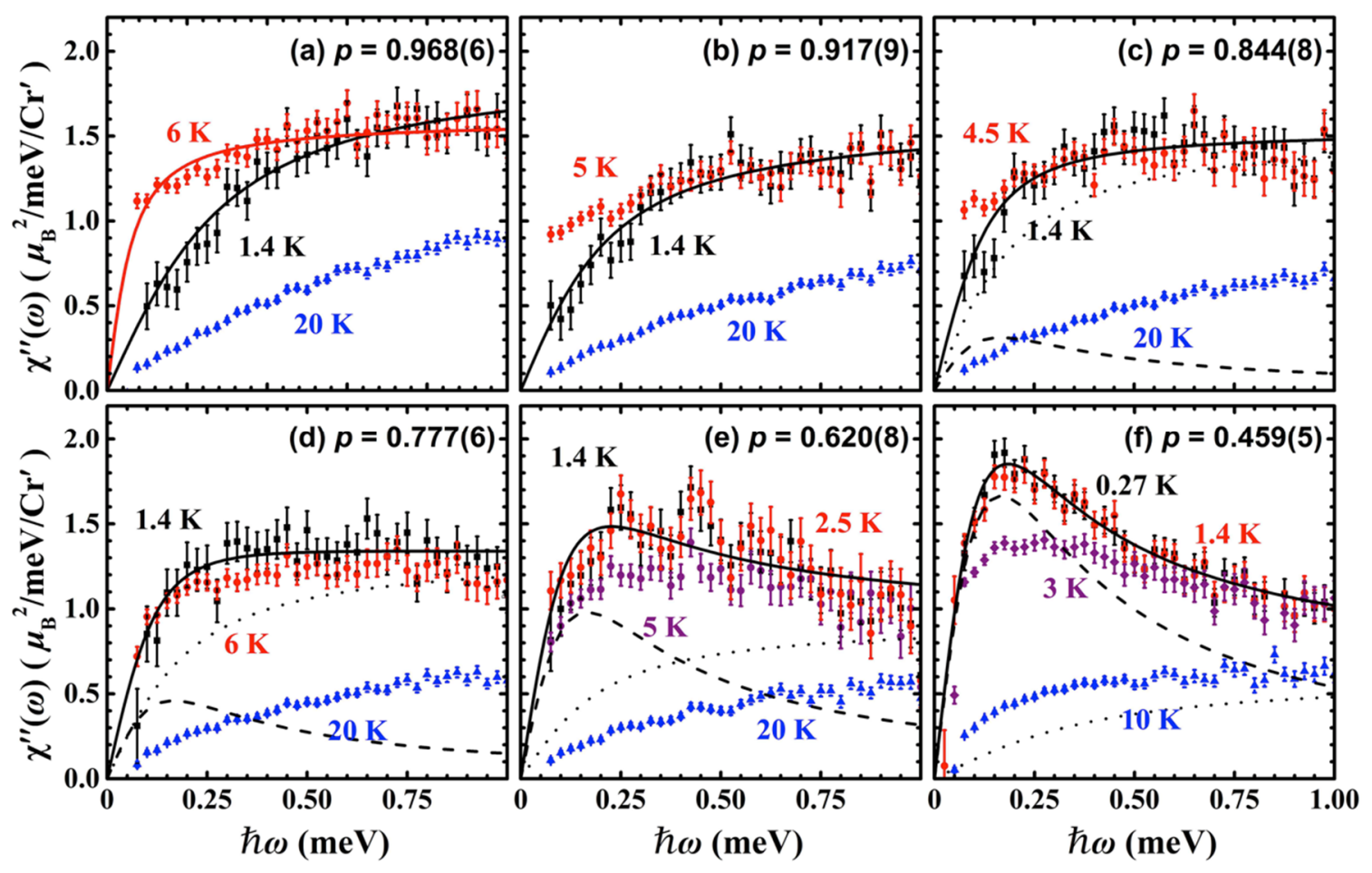

Figure 4.6 $\chi^{\prime \prime}(\omega)$ as a function of temperature and $p$.

The imaginary part of the dynamic susceptibility $\chi^{\prime \prime}(\hbar \omega)$ at low energies was obtained from the inelastic neutron scattering intensities at several different temperatures for various values of $p$ : (a) $p=0.968(6)$, (b) $p=0.917(9)$, (c) $p=0.844(8)$, (d) $p=0.777(6)$, (e) $p=0.620$ (8) and (f) $p=$ $0.459(5)$. In (a), the red solid line is the result of fitting the $6 \mathrm{~K} \sim T_{f}$ data to $\chi^{\prime \prime}(\hbar \omega) \propto \tan ^{-1} \frac{\omega}{\Gamma_{\min }}$ with $\Gamma_{\min }=0.053(4) \mathrm{meV}$. In (a)-(f), the black solid line is the result of fitting the lowest temperature data to $\chi^{\prime \prime}(\omega) \propto\left(\tan ^{-1} \frac{\omega}{\Gamma_{\min }}+f \cdot \frac{\omega}{\omega^{2}+\Gamma_{L}^{2}}\right)$. The dotted and dashed lines are the contributions from arctan and Lorentzian, respectively. In (a) and (b), $f=0$ and the dotted line is under the solid line. For all $p, \Gamma_{\min }$ was determined to be $\Gamma_{\min }=0.25(3) \mathrm{meV} . \Gamma_{L}$ was determined from the $\mathrm{p}=0.459$ data to be $\Gamma_{L}=0.166(3) \mathrm{meV}$ and fixed to the value for all other $\mathrm{p}$. Contributions of each of the two terms, $\tan ^{-1} \frac{\omega}{\Gamma_{\min }}$ and the single Lorentzian, $\frac{\omega}{\omega^{2}+\Gamma_{L}^{2}}$, are plotted as the dotted and the dashed line, respectively.

For $p=0.459(5)$, on the other hand, we observe a fundamentally different behavior in the frozen state: as shown in Figure 4.6 (f), below freezing, rather than hardening, the spectral weight at low frequencies $\chi^{\prime \prime}(\hbar \omega)$ exhibits a prominent increase peaked at $\sim 0.2 \mathrm{meV}$. Obviously, the data cannot be reproduced by $\tan ^{-1} \frac{\omega}{\Gamma_{\min }}$ alone. Instead it behaves more like a Lorentzian with one characteristic relaxation rate, $\chi^{\prime \prime}(\omega) \propto \frac{\omega}{\omega^{2}+\Gamma_{L}^{2}}$. 
The clear difference in the behavior of $\chi^{\prime \prime}(\hbar \omega)$ in the frozen state between the two regimes of $p$ is another evidence that the frozen state in the vicinity of the clean limit is indeed a distinct state. In order to see how the evolution of the state occurs as a function of $p$, we have fitted $\chi^{\prime \prime}(\omega)$ measured in the frozen state of each $p$ to the sum of the two contributions, $\chi^{\prime \prime}(\omega) \propto\left(\tan ^{-1} \frac{\omega}{\Gamma_{\min }}+f \cdot \frac{\omega}{\omega^{2}+\Gamma_{L}^{2}}\right)$. The black solid lines in Figure 4.6 (a)(f) are the fitting results of the sum, while the dotted and dashed lines are the contributions of arctan and Lorentzian, respectively. The fraction of the contribution from the single Lorentzian to $\chi^{\prime \prime}(\omega), f$, was determined in the vicinity to the pure limit to be zero; $f=$ 0.00 (6) for $p=0.968$ and $f=0.00$ (5) for $p=0.917$. As $p$ decreases further, i.e., nonmagnetic doping increases, however, $f$ gradually increases. As shown in Figure 4.6 (c) and (d), the $\tan ^{-1}$ term (dotted line) dominates for $p \gtrsim 0.777(6)$, while the Lorentzian term (dashed line) dominates for $p \lesssim$ 0.7. $f(p)$ over a wide range of $p$ is plotted in Figure 4.5 (c). This confirms, upon doping, a crossover from a frozen state near the clean limit to another frozen state at high nonmagnetic concentration limit.

\subsection{Halperin-Saslow Dynamics}

What is then the nature of the frozen state in the vicinity of the clean limit? The hardening and linear behavior of the low energy spin fluctuations in the frozen state of $\operatorname{SCGO}(p>$ 0.8 ) can be explained as Halperin-Saslow (HS) type modes in a spin jam $[15,16,60]$. HS modes are long-range collective modes which may be viewed as an analog of the Goldstone modes associated with continuous symmetry breaking in systems without long-range order. In contrast, for low values of $p$, as defect concentration is increased, the low-energy spectral weight is eventually dominated by contributions from a distribution of local spin clusters. This is consistent with the previous specific heat data that reported the $C_{v} \propto T^{2}$ behavior robust against dilution for $p \gtrsim 0.8$ (14), while for $p \lesssim 0.8$ the exponent starts decreasing with decreasing $p$ [61].

As a further quantitative evidence of a HS mechanism for the specific heat, $C_{v} / T^{2}$ may be roughly estimated for a spin jam. HS theory with a dispersion $\omega_{k} \cong \gamma \sqrt{\frac{\rho_{s}}{\chi_{0}}} k$, gives a contribution to specific heat $\frac{C v}{\left(k_{B} T\right)^{2}} \cong \frac{9 \zeta(3) k_{B} \chi_{0}}{\pi \hbar^{2} \rho_{s} \gamma^{2}}$, where $\gamma$ is the gyromagnetic ratio, 
$\zeta(3) \sim 1.2$ is the Riemann Zeta function, and we have accounted for 3 modes for isotropic spins. Using experimental values of $\chi_{0} \cong 0.007 \mathrm{emu} / \mathrm{moleCr}$ (see Figure 4.3(a)) and using the HS upper bound estimate $\rho_{s} \cong-\frac{J}{18 V} \sum_{n . n .} r_{i j}^{2}\left\langle S_{i} \cdot S_{j}\right\rangle$ adapted to iSCGO, $J \sim$ $9 \mathrm{meV}$ and $\rho_{s} \cong-\frac{2 J d^{2}}{18 V_{\text {unit cell }}} z\left\langle S^{2}\right\rangle$, taking $|\langle S\rangle|=0.95 \mu_{B} / \mathrm{Cr}$ from Ref. [62], we get a theoretical estimate of $\frac{C v}{T^{2}} \simeq 0.07 \frac{\text { Joule }}{\text { molecr } K^{3}}$, which is consistent with the experimental value of $\sim 0.059 \frac{\text { Joule }}{\text { molecr } K^{3}}$ [37].

\section{4 iSCGO model}

The salient features observed in $\operatorname{SCGO}(p>0.8)$ are consistent with the recent understanding of the iSCGO case with $J=J^{\prime}$ spin jam [5], where $J$ and $J^{\prime}$ are the intra and inter-layer Heisenberg nearest neighbor couplings, respectively. For real SCGO samples $J^{\prime}<J$, in general, due to lattice distortion. For these situations, classical magnetic ground states have been described as a function of $J^{\prime} / J$ in [5]. These can be obtained from the $J=$ $J^{\prime}$ case by coherent rotations of a subset of the spins. The classical manifold of ground states remains degenerate for $J^{\prime}<J$, as can be seen from the flat zero energy bands which are present in the linear spin wave analysis around long range ordered states described in Appendix B . As the ratio $J^{\prime} / J$ changes from 1 to 0 , the system moves from the ideal trilayer to decoupled kagome layers (accompanied by a layer of non-interacting spins), at which point local zero energy modes, such as weathervane modes, associated with kagome physics, appear. For the isolated semi-classical kagome, an extensive configurational entropy of local minima appears with kinetic barriers associated with the weathervane motion. At low enough temperatures tunneling is suppressed and the system freezes. On general grounds, local modes such as the weathervane will become delocalized when the layers are coupled, suggesting that for $J \neq J^{\prime} \neq 0$, as for $J=J^{\prime}$, the barriers between local minima remain non-local in nature and thus freezing is more effective than for the decoupled kagome.

The quantum fluctuations-induced spin jam scenario is consistent with the system freezing at temperatures much lower than $\left|\Theta_{C W}\right|$. An energy scale for spin fluctuations in the clean limit can be determined from the potential barrier between local (ordered) minima and is 
given by $E_{S C G O} \approx 0.05 J S(4)$. For $\operatorname{SCGO}(p>0.8), E_{S C G O} / k_{B} \approx 7.8 \mathrm{~K}$, which is close to the experimentally determined $T_{f} \approx 4 \mathrm{~K} \ll\left|\Theta_{C W}\right| \sim 500 \mathrm{~K}$.

Upon weak non-magnetic doping, the complex energy landscape is modified, the kinetic barriers become finite, however, the overall picture remains the same. Further doping will weaken the order by fluctuations mechanism and the selection of coplanar states, and a different glassy state emerges. Indeed, our observation of a crossover as a function of doping are consistent with Henley [63] who, remarkably, speculated the possibility of a defect-induced crossover as a function of doping, close to the clean limit, from a nongeneric phase (dominated by coplanar states) to a generic spin phase in large spin kagome and SCGO systems.

\subsection{Another dense magnet}

It is worthwhile to discuss in comparison the three-dimensional pyrochlore $\mathrm{Y}_{2} \mathrm{Mo}_{2} \mathrm{O}_{7}$, that was one of the first materials that showed a spin glassy state without non-magnetic impurities [19]. Surprisingly, $\mathrm{Y}_{2} \mathrm{Mo}_{2} \mathrm{O}_{7}$ also exhibits a similar $C_{v} \propto T^{2}$ behavior at low temperatures. [64] The magnetic $\mathrm{Mo}^{4+}\left(4 \mathrm{~d}^{2}\right)$ ions form a three-dimensional network of corner-sharing tetrahedra. If these magnetic moments are isotropic, and antiferromagnetically and uniformly interact with their nearest neighbors only, the system is supposed to yield the highest degree of frustration. One may speculate that the freezing in $\mathrm{Y}_{2} \mathrm{Mo}_{2} \mathrm{O}_{7}$ may also be explained in terms of a spin jam, however we would like to point out crucial differences between the systems. In particular, its frustration index is two orders of magnitude smaller than in SCGO; $f=\frac{\left|\Theta_{C W}\right|}{T_{f}} \approx \frac{45 K}{20 K} \approx 2.3$. This can be understood by the facts that, unlike SCGO which is an excellent insulator, $\mathrm{Y}_{2} \mathrm{Mo}_{2} \mathrm{O}_{7}$ is semi-conducting ( $\rho \sim 10^{-2} \Omega \cdot \mathrm{cm}$ at $300 \mathrm{~K}$ ), and the neighboring Mo ions share one corner of oxygen octahedra, which tends to result in non-negligible longer range magnetic interactions. More importantly, the magnetic $\mathrm{Mo}^{4+}\left(t_{2 g}^{2}\right)$ ions are orbitally degenerate [64,65]; it is well known that orbital degeneracy has a great tendency to modify the nature of a magnetic network, and as a result it reduces dimensionality of the magnetic interactions and frustration as well, as found in $\mathrm{ZnV}_{2} \mathrm{O}_{4}$ [66]. Therefore, the spin glassy state of $\mathrm{Y}_{2} \mathrm{Mo}_{2} \mathrm{O}_{7}$ may be due to spatially random coupling constants induced by the orbital degrees of freedom rather than 
strong frustration. This may explain the experimental observation that $\mathrm{Y}_{2} \mathrm{Mo}_{2} \mathrm{O}_{7}$ resembles conventional spin glasses much more than SCGO. [67] The effectively reduced dimensionality may be related to the observed $C_{v} \propto T^{2}$. Nonetheless, it would be interesting to study effects of nonmagnetic doping on $\mathrm{Y}_{2} \mathrm{Mo}_{2} \mathrm{O}_{7}$ and see if the spin glassy state is robust against small doping as found in SCGO.

\subsection{Conclusion}

The search for glassiness that arises intrinsically without defects and randomness has been revived recently as such glassiness may bear intricate relations with topological order [68], lack of thermalization in many body localization [69], jamming in structural glasses [70] and glassiness in super-cooled liquids [71]. Our experiments indicate that quantum fluctuations, via an "order by disorder" mechanism, induce a glassy state, a spin jam, in the strongly frustrated $\operatorname{SCGO}(p>0.8)$, which is robust and extends to the clean limit. The findings strongly support the possible existence of purely topological glassy states. 


\section{Chapter 5 \\ Spin Jam vs. Spin Glass}

\subsection{Motivation}

The search for glassiness that arises intrinsically without defects and randomness has been revived recently $[4,5]$ as such glassiness may be intricately related to topological order [68], lack of thermalization in many body localization [69], jamming in structural glasses [70] and glassiness in super-cooled liquids [71]. Recently, a microscopic topological mechanism has been proposed for an unconventional frustrated magnet $\mathrm{SrCr}_{9 p} \mathrm{Ga}_{12-9 p} \mathrm{O}_{19}$ (SCGO) in which the magnetic $\mathrm{Cr}^{3+}\left(3 \mathrm{~d}^{3}\right)$ ion surrounded by six oxygen octahedrally, form a distorted quasi-two-dimensional triangular lattice of bi-pyramids [4,5,72]. The mechanism is purely quantum mechanical. The system has a continuous and flat manifold of ground states at the mean field level due to frustration, including locally collinear, coplanar and non-coplanar spin arrangements [4]. Quantum fluctuations lift the classical ground state degeneracy ("order-by-disorder"), resulting in a complex rugged energy landscape that has a plethora of local minima consisting of the locally collinear states separated from each other by potential barriers [5]. As a result, upon cooling below $T_{f}$ set by the energy barriers, the system gets trapped in one of the local minima of collinear bipyramids without long-range order. We refer to this glassy state as spin jam $[5,68]$.

$\mathrm{Y}_{2} \mathrm{Mo}_{2} \mathrm{O}_{7}$ in which the magnetic $\mathrm{Mo}^{4+}\left(4 \mathrm{~d}^{2}\right)$ ions form a three-dimensional network of corner-sharing tetrahedra, is another frustrated magnet which shows a spin glassy state without non-magnetic impurities $[19,20]$. Even though isotropic magnetic moments in an antiferromagnetically coupled pyrochlore network yields a spin liquid state down to low temperatures due to the higher degree of frustration, $\mathrm{Y}_{2} \mathrm{Mo}_{2} \mathrm{O}_{7}$ shown spin freezing 
transition below $T_{f} \sim 20 \mathrm{~K}$. Furthermore, its low-temperature properties are unconventional spin glassy, with a quadratic temperature dependence of the specific heat, $C_{v} \propto T^{2}$ [64], as opposed to the conventional linear behavior. Thus, understanding the nature of the spin frozen state and the microscopic mechanism of the freezing transition of $\mathrm{Y}_{2} \mathrm{Mo}_{2} \mathrm{O}_{7}$ has also been an intellectual challenge in condensed matter physics.

One may speculate that the freezing in $\mathrm{Y}_{2} \mathrm{Mo}_{2} \mathrm{O}_{7}$ may also be explained in terms of a spin jam, however there are crucial differences between $\mathrm{Y}_{2} \mathrm{Mo}_{2} \mathrm{O}_{7}$ and SCGO. In particular, the frustration index of $\mathrm{Y}_{2} \mathrm{Mo}_{2} \mathrm{O}_{7}, f=\frac{\left|\Theta_{C W}\right|}{T_{f}} \approx \frac{45 \mathrm{~K}}{20 \mathrm{~K}} \approx 2.3$, is two orders of magnitude smaller than $f \approx 130$ of SCGO. This can be understood in terms of conductivity where SCGO is an excellent insulator, while $\mathrm{Y}_{2} \mathrm{Mo}_{2} \mathrm{O}_{7}$ is semi-conducting $\left(\rho \sim 10^{-2} \Omega \cdot \mathrm{cm}\right.$ at $\left.300 \mathrm{~K}\right)$, and the neighboring Mo ions share one corner of oxygen octahedra, which tends to result in non-negligible longer range magnetic interactions. More importantly, the magnetic $\mathrm{Mo}^{4+}$ $\left(t_{2 g}^{2}\right)$ ions are orbitally degenerate $[64,65]$; it is well known that orbital degeneracy has a tendency to modify the nature of a magnetic network, and as a result it reduces the dimensionality of the magnetic interactions and frustration. This is the case of $\mathrm{ZnV}_{2} \mathrm{O}_{4}$ [66]. Therefore, we believe that the spin glassy state of $\mathrm{Y}_{2} \mathrm{Mo}_{2} \mathrm{O}_{7}$ may be due to spatially random coupling constants induced by the orbital degrees of freedom rather than strong frustration. The effectively reduced dimensionality may be related to the observed $C_{v} \propto T^{2}$ behavior.

To investigate the nature of the spin frozen states and the microscopic mechanisms of the freezing transitions from frustrated lattices in the defects free limit, we have performed bulk susceptibility and neutron scattering experiments on two geometrically frustrated magnetic glass systems: $\mathrm{Y}_{2} \mathrm{Mo}_{2} \mathrm{O}_{7}$ and $\operatorname{BCGO}(p=0.907(8)) / \operatorname{SCGO}(\mathrm{p}=0.968(6))$ in comparison to an archetypical spin glass $\mathrm{CuMn} 2 \%$. 


\subsection{Bulk Susceptibility Experiments}

Here, we study the bulk susceptibility of $\mathrm{CuMn} 2 \%, \operatorname{BCGO}(p=0.902(8))$ and $\mathrm{Y}_{2} \mathrm{Mo}_{2} \mathrm{O}_{7}$ samples as summarized in Figure 5.1. All the three samples were measured for directcurrent $(\mathrm{DC})$ susceptibility $\left(\chi_{D C}\right)$ after cooling to the base temperature $\left(T_{\text {base }}\right)$ of the instrument with and without a magnetic field about 100 Oersted (Oe). In all the three systems, the zero-field cooled susceptibility $\left(\chi_{Z F C}\right)$ shows a cusp at low temperature implying a magnetic transition at the cusp temperature $\left(T_{D C}\right)$ while the field-cooled susceptibility $\left(\chi_{F C}\right)$ shows a saturation below $T_{D C}$ by creating a low temperature hysteresis. The ZFC-FC bifurcation shows a direct evidence of ergodicity breaking in these systems at least within the measuring time scale of $\chi_{D C}(\sim 100 \mathrm{~s})$.
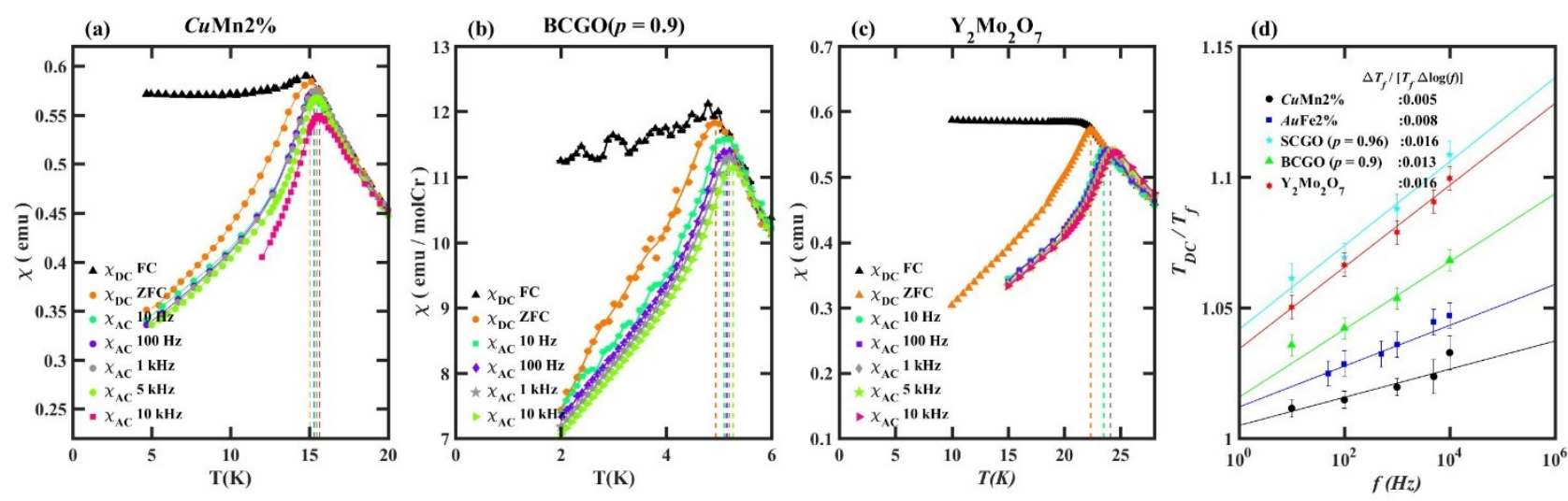

Figure 5.1 Bulk Susceptibility of $C u M n 2 \%, \operatorname{BCGO}(p=0.902(8))$ and $\mathrm{Y}_{2} \mathrm{Mo}_{2} \mathrm{O}_{7}$

DC susceptibility $\left(\chi_{D C}\right)$ under zero-field-cooled (ZFC) and field-cooled (FC) conditions, and AC susceptibility $\left(\chi_{A C}\right)$ at different frequencies are measured on (a) $C u \mathrm{Mn} 2 \%$, (b) $\mathrm{BCGO}(p=0.902(8))$ and (c) $\mathrm{Y}_{2} \mathrm{Mo}_{2} \mathrm{O}_{7}$ samples. The freezing temperature $T_{f}$ is determined measurements of Y2Mo2o7 are summarized here. $\chi_{D C}$ was measured with an applied field of 10 oe while the AC measurements were performed under zero static field and amplitude of $15 \mathrm{oe}$. The insert shows the cusp temperature $T_{f}$ as a function of frequency and $T_{D C}$ is estimated as $22.34(5) \mathrm{K}$.

The alternating current $(\mathrm{AC})$ susceptibility $\left(\chi_{A C}\right)$ has also measured at different frequencies of the applied alternating magnetic field, oscillating around the remnant static field at the sample space. For all the three systems, $\chi_{A C}$ also shows a cusp similar to the $\chi_{Z F C}$ but the cusp temperature $\left(T_{f}\right)$ is been shifted to the higher temperatures and the shift is increasing with increasing frequency of the field. The frequency dependence of $T_{f}$ can be expected when a magnetic system has multiple relaxations at low temperature which is a typical 
nature of spin glasses, spin liquids, Superparamagnets or cooperative paramagnets [7]. Figure 5.1 (d) summarizes the frequency dependence of the $T_{f}$ for many different magnets: two archetypical spin glasses, $C u \mathrm{Mn} 2 \%$ and $A u \mathrm{Fe} 2 \%$, two Quasi-2D frustrated magnets, $\operatorname{SCGO}(p=0.968(6))$ and $\operatorname{BCGO}(p=0.902(8))$, and a 3D pyrochlore system, $\mathrm{Y}_{2} \mathrm{Mo}_{2} \mathrm{O}_{7}$. The solid lines in the Figure $5.1(\mathrm{~d})$ are the fits of $T_{f}$ to a function $\log \left(T_{f}\right)=m \log (f)+$ $b$, where $m=\Delta T_{f} /\left(T_{f} \Delta \log (f)\right)$ and the parameter $m$ is usually been used to categorize different magnetic systems primarily [7]. Both $C u \mathrm{Mn} 2 \%$ and $A u \mathrm{Fe} 2 \%$ shows very low values for $\Delta T_{f} /\left(T_{f} \Delta \log (f)\right)$ as expected for dilute spin glasses and the values are consistent with literature [7]. $\mathrm{But} \operatorname{SCGO}(p=0.968(6)), \mathrm{BCGO}(p=0.902(8))$ and $\mathrm{Y}_{2} \mathrm{Mo}_{2} \mathrm{O}_{7}$ shows slightly higher $\Delta T_{f} /\left(T_{f} \Delta \log (f)\right)$ by indicating a deviation from conventional spin glass behavior. To get a clear picture, we need to study freezing behavior in a much broader time scales.

\subsection{Neutron Scattering Experiments}

In this section, we discuss the neutron scattering experiments done on the three samples $\operatorname{SCGO}(p=0.968(6)), \quad \operatorname{BCGO}(p=0.902(8))$ and $\mathrm{Y}_{2} \mathrm{Mo}_{2} \mathrm{O}_{7}$, using various scattering techniques such as Neutron Spin Echo (NSE), Neutron Backscattering and Neutron Timeof-Flight. From the neutron scattering experiments, we have access to both spatial and temporal information of a particular magnet. In this chapter, we will mainly focus on the dynamical properties rather than the static nature of systems above.

\subsubsection{Neutron Spin Echo measurements}

Here, we study the intermediate scattering function $I\left(Q, \tau_{0}\right)$ at a fixed wavevector $Q$ and a fixed Fourier time $\tau_{0}$, as a function temperature on the three magnetic glasses; $\operatorname{BCGO}(p=$ $0.902(8)), \mathrm{Y}_{2} \mathrm{Mo}_{2} \mathrm{O}_{7}$ and $C u \mathrm{Mn} 2 \%$. Both $C u \mathrm{Mn} 2 \%$ and $\mathrm{BCGO}(p=0.902(8))$ samples were

measured at NSE spectrometer, NIST with initial wavelength of $\lambda=6 \AA$ (bandwidth, $\Delta \lambda / \lambda \sim 20 \%)$. As shown in the Figure 5.2 (a), $I\left(Q, \tau_{0}, T\right)$ of $C u \mathrm{Mn} 2 \%$ was measured at $Q=0.2 \AA^{-1}$ and $\tau_{0}=5 \mathrm{~ns}$ on a $\sim 30 \mathrm{~g}$ sample. For $\operatorname{BCGO}(p=0.902(8)), I\left(Q, \tau_{0}, T\right)$ was measured at $Q=1.5 \AA^{-1}$ and at two Fourier times, $5 \mathrm{~ns}$ and $0.05 \mathrm{~ns}$ (see Figure 5.2 (b)). The spin echo measurements on $\mathrm{Y}_{2} \mathrm{Mo}_{2} \mathrm{O}_{7}$ was performed at NSE spectrometer, Spallation 
Neutron Source (SNS), at Oak Ridge National Laboratory (ORNL). The $I\left(Q, \tau_{0}, T\right)$ for $\mathrm{Y}_{2} \mathrm{Mo}_{2} \mathrm{O}_{7}$ was measured at $Q=0.45 \AA^{-1}$ and Fourier time in the range of $0.1 \mathrm{~ns}<\tau_{0}<$ $1 \mathrm{~ns}$. Since this spectrometer is at a spallation source, a measurement at a single set of instrument setting can capture a broad range of F.T. and we have rebinned the time channel to extract $I\left(Q, \tau_{0}, T\right)$ at $\tau_{0}=0.33 \mathrm{~ns}$ and $0.55 \mathrm{~ns}$. (see Figure $5.2(\mathrm{c})$ )
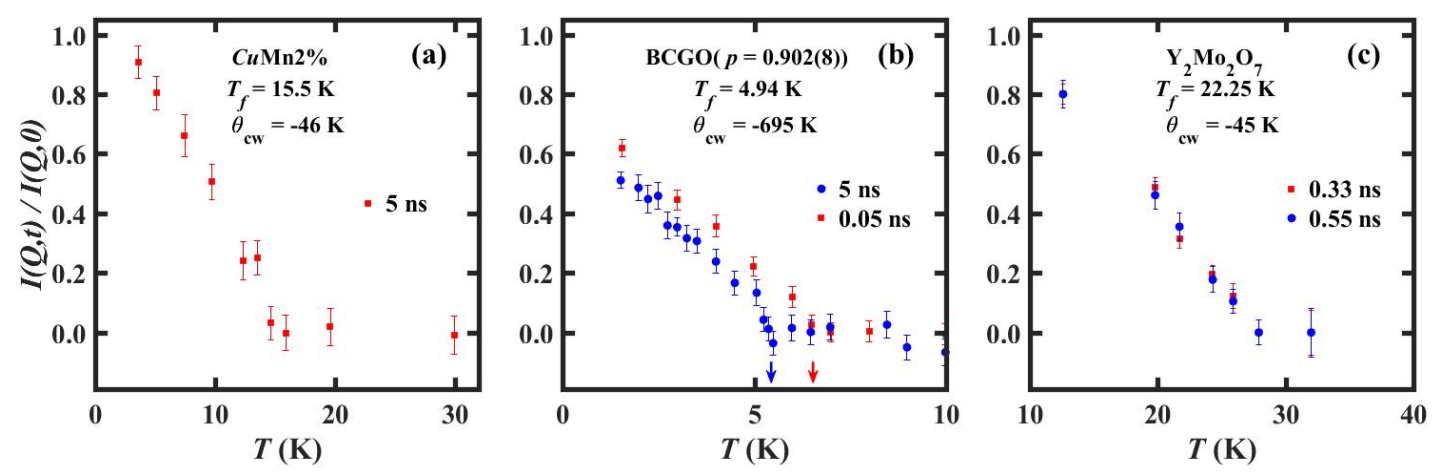

Figure 5.2 Magnetic order-parameters from Neutron Spin Echo

The normalized intermediate scattering function $I\left(Q, \tau_{0}\right)$ on (a) $C u \mathrm{Mn} 2 \%$ at $Q_{0}=0.2 \AA^{-1}$ and $\tau_{0}=5 \mathrm{~ns}$, (b) $\operatorname{BCGO}(p=0.902(8))$ at $Q_{0}=1.5 \AA^{-1}$ and $\tau_{0}=0.05,5 \mathrm{~ns}$, and (c) $\mathrm{Y}_{2} \mathrm{Mo}_{2} \mathrm{O}_{7}$ at $Q_{0}=0.45 \AA^{-1}$ and $\tau_{0}=0.33,0.55 \mathrm{~ns}$, has been measured. $C u \operatorname{Mn} 2 \%$ and $\operatorname{BCGO}(p=0.902(8))$ samples were measured at NSE spectrometer at NCNR, NIST while $\mathrm{Y}_{2} \mathrm{Mo}_{2} \mathrm{O}_{7}$ was measured at NSE spectrometer at SNS, ORNL.

The intermediate scattering function at a fixed wavevector $Q_{0}, I\left(Q_{0}, t\right)$ can be written as,

$$
I\left(Q_{0}, t\right)=\int_{-\infty}^{\infty} S\left(Q_{0}, \omega\right) e^{-i \omega t} d \omega
$$

When $S\left(Q_{0}, t\right)$ is a slowly varying function, which is typically the case for magnetic glasses, the Equation 5.1 can be simplified as,

$$
I\left(Q_{0}, t\right)=\int_{-\Delta \omega}^{\Delta \omega} S\left(Q_{0}, \omega\right) d \omega
$$

where $\Delta \omega$ is the instrument resolution defined by the Fourier time $t=h / \Delta \omega$. From another perspective, $I\left(Q_{0}, t=\tau_{0}\right)$ at a fixed Fourier time $\tau_{0}$, accounts all the relaxations slower than $\tau_{0}\left(\tau>\tau_{0}\right)$ as static or frozen and ignores all the relaxations faster than $\tau_{0}\left(\tau<\tau_{0}\right)$. Thus, $I\left(Q_{0}, \tau_{0}, T\right)$ can be used to measure the elastic order-parameter of a magnetic glasses for given resolution of $\Delta \omega_{0}=h / \tau_{0}$. 
From Equation 3.56 of ref. [7], the susceptibility, corresponding to a particular relaxation time $\tau_{0}$ and wave vector $Q_{0}, \chi\left(Q_{0}, \tau_{0}\right)$ can be written as,

$$
\chi\left(Q_{0}, \tau_{0}, T\right) \propto \frac{1}{T}\left[1-I\left(Q_{0}, \tau_{0}, T\right)\right]
$$

To estimate the freezing temperature at a particular F.T., we calculated the corresponding susceptibility $\chi\left(Q_{0}, \tau_{0}, T\right)$ using Equation 5.3 and fitted the data to a smoothing spline and determined $T_{f}$ as the cusp temperature. (see Figure (App.) A.5 for $\operatorname{BCGO}(p=0.902(8)$, Figure (App.) $\quad$ A.6 for $C u \mathrm{Mn} 2 \%$ and Figure (App.) A.7 for $\mathrm{Y}_{2} \mathrm{Mo}_{2} \mathrm{O}_{7}$ ). The $T_{f}$ for $\mathrm{CuMn} 2 \%$ at $5 \mathrm{~ns}$ is $15.4(5) \mathrm{K}$ which is comparable with the bulk susceptibility measurements with in the estimated uncertainty. For BCGO, $T_{f} \mathrm{~s}$ for $\tau_{0}=5 \mathrm{~ns}$ and $0.05 \mathrm{~ns}$ are 5.53(17) $\mathrm{K}$ and $6.92(18) \mathrm{K}$ which are slightly higher than $T_{f}=4.94 \mathrm{~K}$, measured from DC susceptibility. Notice that there is also a significant shift between $T_{f}$ s of two F.T.s. The shift of $T_{f}$, even within the nanoseconds for $\operatorname{BCGO}(p=0.902(8))$ give us more clues that BCGO is different from $C u \mathrm{Mn} 2 \%$. Moreover, the $T_{f}$ s for $\mathrm{Y}_{2} \mathrm{Mo}_{2} \mathrm{O}_{7}$ at $\tau_{0}=0.55 \mathrm{~ns}$ and $0.33 \mathrm{~ns}$ are estimated as 28.1(1.4) $\mathrm{K}$ and 28.31(1.4) $\mathrm{K}$ respectively. These $T_{f} \mathrm{~s}$ are also significantly higher values than the cusp temperature of 22.34(4) K, measured in Bulk susceptibility (see Figure 5.1 (c)). Apparently, the magnetic glass phases from the dense limit of geometrically frustrated magnets has similarities regardless of geometrical differences, yet distinct from conventional spin glass behavior of $C u \mathrm{Mn} 2 \%$. 


\subsubsection{Backscattering spectroscopy}

To cover the $\mu \mathrm{eV}$ energy resolutions, we have performed backscattering spectroscopy on $\sim 10 \mathrm{~g}$ samples of $C u \mathrm{Mn} 2 \%, \mathrm{BCGO}(p=0.902(8))$ and $\mathrm{Y}_{2} \mathrm{Mo}_{2} \mathrm{O}_{7}$ at BASIS spectrometer at SNS, ORNL. During the experiments, the instrument was configured to a neutron beam of wavelength $\lambda=6.4 \AA$ and frequency $30 \mathrm{~Hz}$ to achieve the instrument resolution of $4 \mu \mathrm{eV}$, dynamical range of $-250 \mu \mathrm{eV} \leq E \leq 250 \mu \mathrm{eV}$ and $Q$ range of $0.3 \AA^{-1} \leq Q \leq 2 \AA^{-1}$.
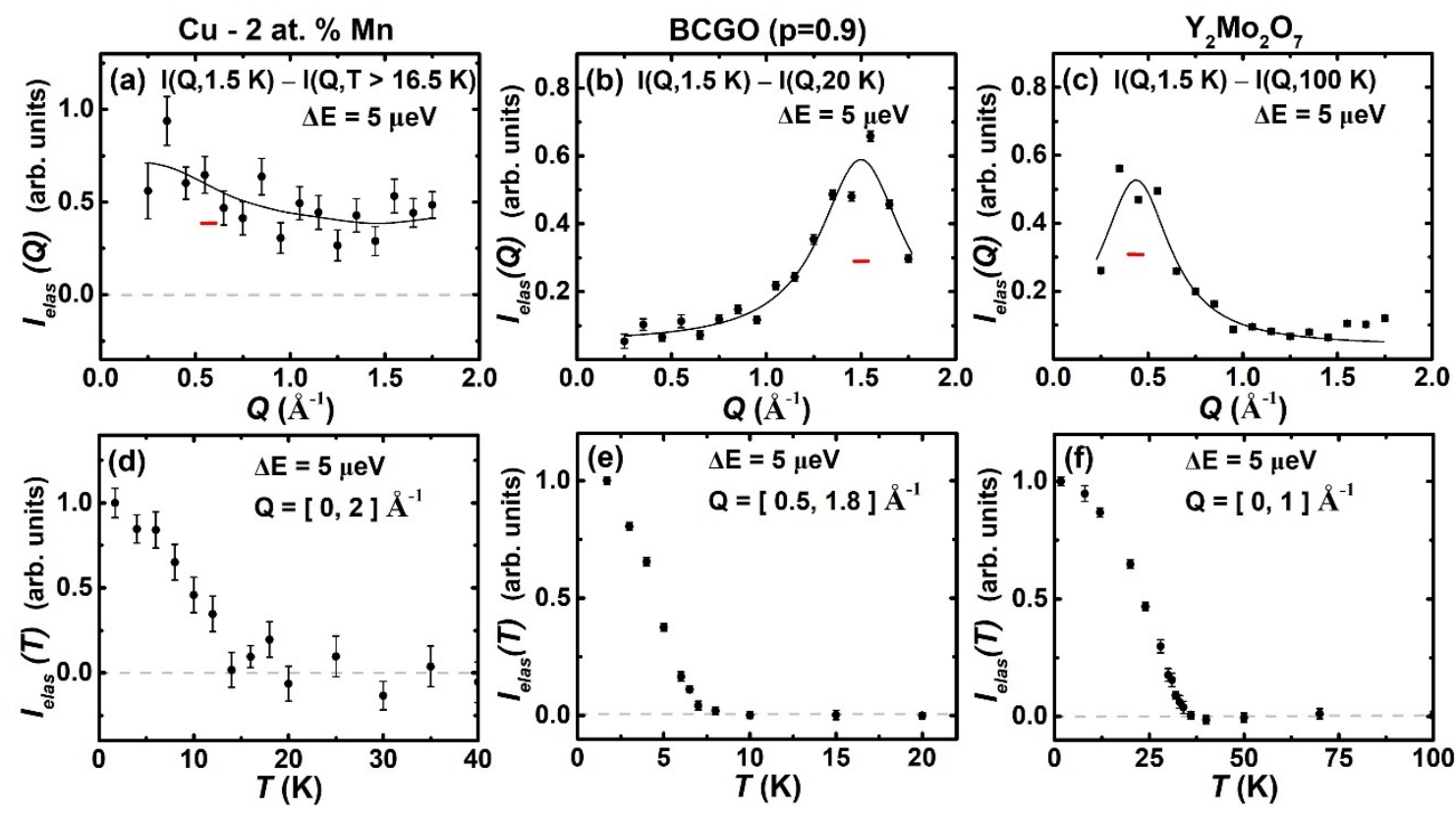

Figure 5.3 Neutron Backscattering experiments of $\mathrm{BCGO}(p=0.9), \mathrm{Y}_{2} \mathrm{Mo}_{2} \mathrm{O}_{7}$ and $\mathrm{CuMn2 \%}$

(a) $Q$-dependence, $I_{\text {elas }}(Q)$, and (d) T-dependence, $I_{\text {elas }}(T)$, of elastic magnetic neutron scattering intensity obtained from $C u \mathrm{Mn} 2 \%$. (b) $Q$-dependence, $I_{\text {elas }}(Q)$, and (e) T-dependence, $I_{\text {elas }}(T)$, of elastic magnetic neutron scattering intensity obtained from $\operatorname{BCGO}(p=0.902(8))$. (c) $Q$-dependence, $I_{\text {elas }}(Q)$, and (f) T-dependence, $I_{\text {elas }}(T)$, of elastic magnetic neutron scattering intensity obtained from $\mathrm{Y}_{2} \mathrm{Mo}_{2} \mathrm{O}_{7}$. The measurements were done at the Backscattering Spectrometer (BASIS) at SNS. For $I_{\text {elas }}(Q)$ in (a), (b) and (c), the non-magnetic background was determined by the measurements at higher temperatures above the corresponding freezing temperature and subtracted from the base temperature data. The black solid lines in (b) and (c) are the fits of the magnetic peaks centered at $Q=1.50(2) \AA^{-1}$ and $Q=0.44(2) \AA^{-1}$ respectively to a simple Lorentzian while it is an eye guide to the data in (a). The $Q$ resolution of the instrument is also marked by the red horizontal lines in (a), (b) and (c).

Figure 5.3 (a), (b) and (c) show the $Q$ dependence of magnetic neutron scattering crosssection, $I_{m a g}(Q)=\int_{-\Delta \omega_{0}}^{\Delta \omega_{0}} I_{m a g}(Q, \omega) d \omega$ within the instrumental resolution of $\Delta \omega_{0}=$ $4 \mu \mathrm{eV}$. Here the magnetic component of the signal $\left(I_{\text {mag }}(Q, \omega)\right)$ was calculated by 
estimating the non-magnetic scattering background $\left(I_{B G}\right)$ at higher temperatures $\left(T_{\text {high }}\right)$ above corresponding freezing temperatures $\left(T_{f}\right)$ and subtracting it from the low temperature measurements. For $\mathrm{CuMn} 2 \%, I_{B G}(Q)$ was determined by the data collected at temperatures higher than $16.5 \mathrm{~K}\left(>T_{f}=15.5 \mathrm{~K}\right)$ while it is at $20 \mathrm{~K}\left(>T_{f}=4.94 \mathrm{~K}\right)$ for $\operatorname{BCGO}(p=0.902(8))$ and at $100 \mathrm{~K}\left(>T_{f}=22.25 \mathrm{~K}\right)$ for $\mathrm{Y}_{2} \mathrm{Mo}_{2} \mathrm{O}_{7}$. As shown in Figure 5.3 (a), $I_{m a g}(Q)$ of $C u \mathrm{Mn} 2 \%$ shows a nearly featureless $Q$ dependence with maximum intensity as $Q \rightarrow 0$. In the dilute magnetic alloys such as $C u \mathrm{Mn} 2 \%$, magnetic impurities interact among themselves through the Ruderman-Kittel-Kasuya-Yosida (RKKY) interactions that are mediated by the itinerant electrons. The RKKY interactions are longranged, and oscillate from ferromagnetic to antiferromagnetic as a function of the distance. As a result, the random distances among the magnetic moments lead to their random interactions that even change the sign, resulting in the featureless magnetic structure factor.

In contrast, the two frustrated magnets, $\mathrm{BCGO}(p=0.902(8))$ and $\mathrm{Y}_{2} \mathrm{Mo}_{2} \mathrm{O}_{7}$, shows prominent peaks centered around finite wavevectors $Q_{\max } \approx 1.5 \AA^{-1}$ and $0.4 \AA^{-1}$ respectively. This experimental observation is consistent previous published neutron data of $\mathrm{BCGO}$ and $\mathrm{Y}_{2} \mathrm{Mo}_{2} \mathrm{O}_{7}$ at different energy resolutions. The diffusive peak in $Q-$ dependence of elastic neutron scattering is corresponding to dominant antiferromagnetic interactions between localized spins and short-range spin correlations in these frustrated magnets. Moreover, $Q_{\max }$ is the characteristic wavevector of crystallographic unit responsible for magnetic glass-like freezing. For example, in the case of $\operatorname{BCGO}(p=$ $0.902(8)), \quad Q_{\max } \approx 1.5 \AA^{-1}$ is corresponds to $(2 / 3,2 / 3,1.8)$, confirming that the kagome-triangular-kagome trilayer is responsible. However, the $Q_{\max } \approx 0.4 \AA^{-1}$ in the case of $\mathrm{Y}_{2} \mathrm{Mo}_{2} \mathrm{O}_{7}$ is not yet clearly understood.

As shown in Figure $5.3(\mathrm{~d}),(\mathrm{e})$ and (f), the magnetic order-parameter $I\left(Q_{0}, \tau_{0}, T\right)$ was calculated by,

$$
I\left(Q_{0}, \tau_{0}, T\right)=\int_{-\Delta \omega_{0}}^{\Delta \omega_{0}} \int_{Q_{\min }}^{Q_{\max }} I_{\operatorname{mag}}(Q, \omega, T) d Q d \omega
$$

where $\Delta \omega_{0}=h / \tau_{0}=4 \mu \mathrm{eV}\left(\tau_{0}=1 \mathrm{~ns}\right)$ and $\Delta Q=\left[Q_{\min }, Q_{\max }\right]$ was selected to cover the peak magnetic intensity for each sample. The meaning of the $I\left(Q_{0}, \tau_{0}, T\right)$ is as explained 
in the Chapter 5.3.1 in which the $I\left(Q_{0}, \tau_{0}, T\right)$ is coming from the relaxations slower than the cut-off relaxation time $\tau_{0}$, set by the instrument resolution $\Delta \omega_{0}$. Actually, $\Delta \omega_{0}$ is the minimum energy resolution that we can achieve by the current instrument setup. Indeed, we can always calculate order-parameters at higher $\omega$ integration ranges as large as the entire dynamical range of the instrument which is $\pm 250 \mu \mathrm{eV}$. (see Figure 5.4).

To determine the freezing temperature $\left(T_{f}\right)$ at a particular energy resolution $\Delta \omega_{0}$, we have calculated the corresponding susceptibility using Equation 5.3, similar to the analysis we did with NSE measurements. The estimated $T_{f}(4 \mu \mathrm{eV}) \mathrm{s}$ are $6.14(25) \mathrm{K}$ for $\operatorname{BCGO}(p=$ $0.902(8)$ ) and 33.2(5) $\mathrm{K}$ for $\mathrm{Y}_{2} \mathrm{Mo}_{2} \mathrm{O}_{7}$. Even though, the BASIS measurements of $\mathrm{CuMn} 2 \%$ shows a clear elastic orderparameter as shown in the Figure 5.3 (d), the statics are not good enough to estimate $T_{f}(4 \mu \mathrm{eV})$ by mean of the systematic analysis we did, for other data sets. However, we can roughly estimate the $T_{f}$ as $\sim 15 \mathrm{~K}$ just by looking at the Figure 5.3 (d) and it is consistent with previously measured Bulk susceptibility and NSE measurements. Notice that $T_{f}(4 \mu \mathrm{eV})_{\mathrm{s}}$ for $\mathrm{BCGO}(p=0.902(8))$ and $\mathrm{Y}_{2} \mathrm{Mo}_{2} \mathrm{O}_{7}$ are significantly different from corresponding Bulk susceptibility and NSE measurements, in contrast to the $\mathrm{CuMn} 2 \%$.

To verify this behavior of $C u \mathrm{Mn} 2 \%$, another backscattering experiment was done for $\mathrm{CuMn} 2 \%$ at High Flux Backscattering Spectrometer (HFBS) at NIST Center for Neutron Research on a bigger sample of $30 \mathrm{~g}$. In the typical operation of HFBS, the continuous incoming beam of a wavelength of $6.271 \AA$ from the NCNR reactor is illuminated on an oscillating doppler arm at $20 \mathrm{~Hz}$ to achieve a dynamical range of $-15 \mu \mathrm{eV} \leq \omega \leq 15 \mu \mathrm{eV}$ by vary the energy of incident neutrons at the sample. The energy resolution of the instrument is $\Delta \omega \sim 0.8 \mu \mathrm{eV}$, set by the silicon analyzer crystals. For better statistics, we have fixed the doppler arm, hence called fixed window scan and measured neutron scattering intensity $I(Q, T)$ as a function of wavevector $(Q)$ and temperature $(T)$ on $C u \mathrm{Mn} 2 \%$ as shown in the Figure 5.4 (a) (look for maroon color diamonds). Thus the $T_{f}$ at $0.8 \mu \mathrm{eV}$ was estimated as $15.57(35) \mathrm{K}$ for $C u \mathrm{Mn} 2 \%(\chi(\tau=0.8 \mu \mathrm{eV}, T)$ is shown in the Figure (App.) A.6 (a)). Thus, the HFBS experiment confirms that the $T_{f}$ at $\mu \mathrm{eV}$-resolution is comparable to the Bulk susceptibility in the case of $C u \mathrm{Mn} 2 \%$. 


\subsection{Spectral Distribution of Relaxation Time}

In the previous sections, we have studied the freezing temperature $T_{f}\left(\Delta \tau_{0}\right)$ as a function of cut-off relaxation time $\tau_{0}=h / \Delta \omega_{0}$ from Bulk Susceptibility (Typically $\left(\tau_{0}\right)_{D C} \approx 100 \mathrm{~s}$ ) to Backscattering spectroscopy $\left(\left(\tau_{0}\right)_{\min } \approx 1 \mathrm{~ns}\right)$ for $\mathrm{CuMn} 2 \%, \operatorname{BCGO}(p=0.902(8))$ and $\mathrm{Y}_{2} \mathrm{Mo}_{2} \mathrm{O}_{7}$ magnets. To get much broader $\tau_{0}$ dependence, we have performed the Neutron time-of -flight measurements on $\sim 10 \mathrm{~g}$ samples of $\mathrm{BCGO}(p=0.902(8))$ and $\mathrm{Y}_{2} \mathrm{Mo}_{2} \mathrm{O}_{7}$ at Disk Chopper Spectrometer (DCS), NCNR with neutron wave lengths of $\lambda=6 \AA$ and $\lambda=$ $8 \AA$ respectively. The main features of $\operatorname{BCGO}(p=0.902(8))$ time-of-flight spectroscopy are already discussed in the Chapter 3.6 and order-parameters at different $\Delta \omega_{0}$ ranging from the instrument energy resolution at $\lambda=6 \AA, \Delta \omega_{0}=35 \mu \mathrm{eV}$ to $\Delta \omega_{0}=200 \mu \mathrm{eV}$ are summarized in the Figure 5.4 (b). The estimated $T_{f}\left(\Delta \omega_{0}\right) \mathrm{s}$ at $\Delta \omega_{0}=35 \mu \mathrm{eV}$ and $\Delta \omega_{0}=$ $200 \mu \mathrm{eV}$ for the $\mathrm{BCGO}(p=0.902(8))$ are $7.6(6) \mathrm{K}$ and $10.5(8) \mathrm{K}$ respectively.

In the case of $\mathrm{Y}_{2} \mathrm{Mo}_{2} \mathrm{O}_{7}$, we have observed a continuum spectrum centered in the vicinity of $Q_{\max } \approx 0.45 \AA^{-1}$ similar to the continuum at $Q_{\max } \approx 1.5 \AA^{-1}$ in case of $\operatorname{SCGO}(p=$ $0.968(6))$ or $\mathrm{BCGO}(p=0.902(8))$. Even though, the $Q_{\max }$ in $\mathrm{Y}_{2} \mathrm{Mo}_{2} \mathrm{O}_{7}$ is not yet completely understood unlikely in SCGO/BCGO systems, here we study the elastic orderparameter, $I\left(Q_{0}, \tau_{0}, T\right)$ as a function $\Delta \omega_{0}$ in the range of $35 \mu \mathrm{eV} \leq \Delta \omega_{0} \leq 120 \mu \mathrm{eV}$ as shown in the Figure 5.4 (c). Thus the $T_{f}$ at $\tau_{0}=30 \mu \mathrm{eV}$ and $120 \mu \mathrm{eV}$, and $Q_{0}=[0,2] \AA^{-1}$ were estimated as 31(1) K and 36(2) K respectively. (see Figure (App.) A.7 (a) for corresponding $\chi\left(Q_{0}, \tau_{0}, T\right)$ calculations $)$.

All the elastic order-parameters $I\left(Q_{0}, \Delta \omega_{0}, T\right)$ measured at different $\Delta \omega_{0}$ are summarized in Figure 5.4 (a), (b) and (c) for $C u \mathrm{Mn} 2 \%, \operatorname{BCGO}(p=0.902(8))$ and $\mathrm{Y}_{2} \mathrm{Mo}_{2} \mathrm{O}_{7}$ samples respectively. Moreover, Figure 5.4 (d), (e) and (f) shows the contour maps of $I\left(Q_{0}, \Delta \omega_{0}, T\right)$ as a function of $\Delta \omega_{0}$ and $T$. The $I\left(Q_{0}, \Delta \omega_{0}, T\right)$ corresponding to bulk measurements $\left(\chi_{D C}\right.$ or $\left.\chi_{A C}\right)$ has been calculated using the Equation 5.3. In Figure 5.4, all the $I\left(Q_{0}, \Delta \omega_{0}, T\right) \mathrm{s}$ are normalized to $I\left(Q_{0}, \Delta \omega_{0}, T=0\right)$ estimated by extrapolating each dataset. The normalized $I\left(Q_{0}, \Delta \omega_{0}, T\right)$ represents the spectral density of relaxations longer than the cut-off relaxation time $\tau_{0}=h / \Delta \omega_{0}$ defined by the elastic energy window $\pm \Delta \omega_{0}$ as percentage to the total scattering. Notice that the total magnetic scattering of each compound estimated 
by the polarization analysis, are also presented in Figure 5.4 (black triangles in each panel) and they are nearly flat over the entire measuring range of temperature while the integrated scattering intensities, $I\left(Q_{0}, \Delta \omega_{0}, T\right)$ at a particular $\Delta \omega_{0}$ shows less intensity at higher temperature. Furthermore, the $I\left(Q_{0}, \Delta \omega_{0}, T\right)$ at larger $\Delta \omega_{0}$, starts building up at higher temperatures upon cooling in comparison to larger $\Delta \omega_{0}$, by showing a frequency dependence of freezing behaviors.
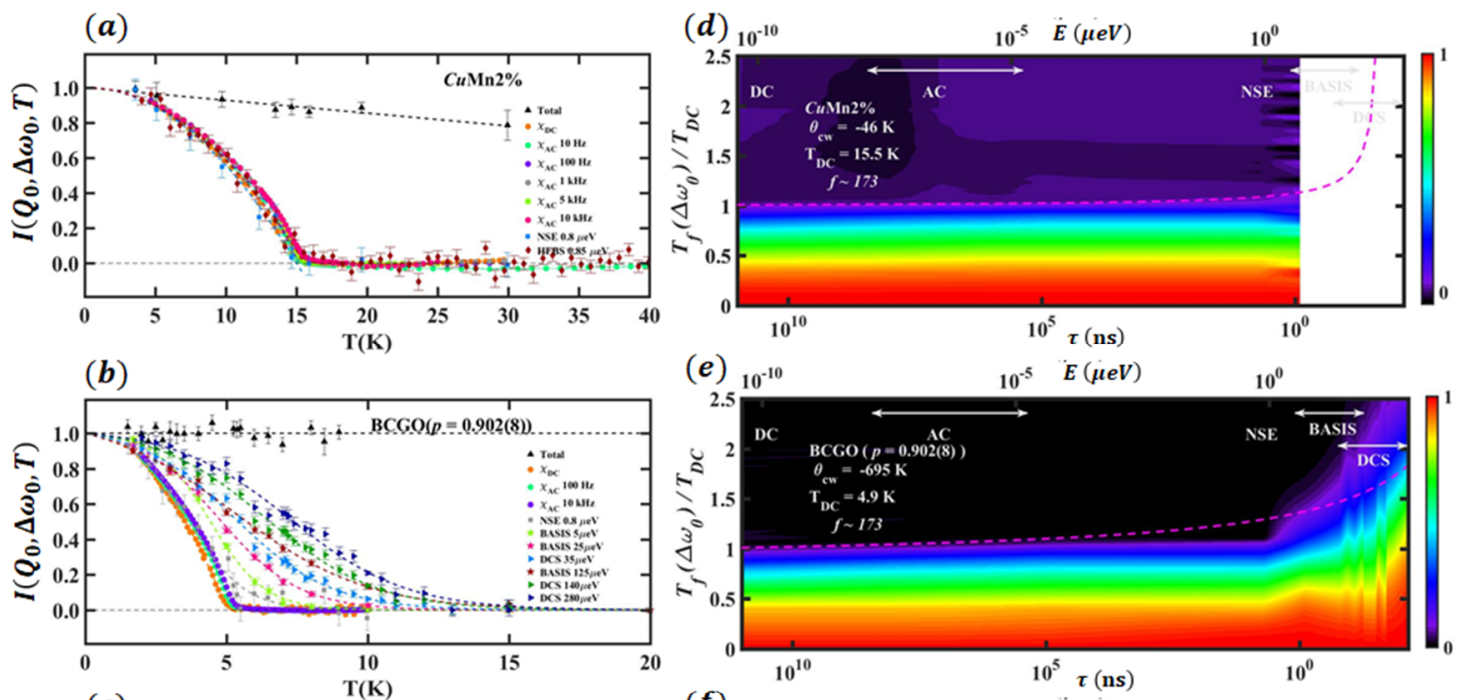

(c)

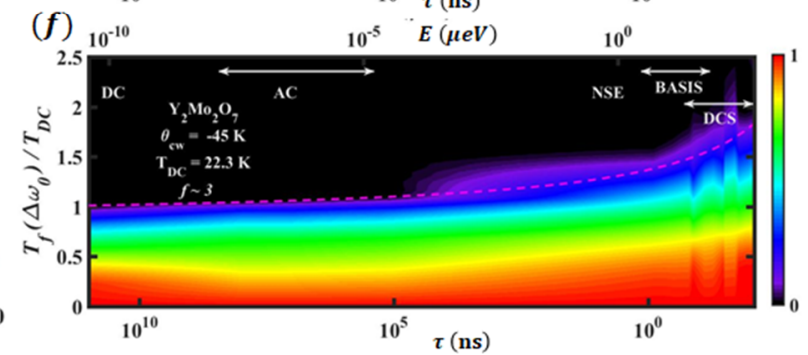

Figure 5.4 Summery of elastic order parameter

Elastic order parameter as function of temperature and energy resolution for (a) $\mathrm{CuMn} 2 \%$, (b) $\mathrm{BCGO}(p=$ $0.902(8)$ ) and (c) Y2Mo2O7 systems and the corresponding contour maps are shown in (d), (e) and (f) respectively. The Magenta color dashed lines in (d), (e) and (f), are the fittings of $T_{f}\left(\Delta \omega_{0}\right)$ shown in the Figure 5.5 (a).

To Further analyze the dynamics at different energy scales, we have calculated inelastic order-parameter defined by the integral of $\varepsilon_{\text {inelastic }}(\delta \omega, T)=\int_{\omega_{1}}^{\omega_{2}} I\left(Q_{0}, \omega, T\right) d \omega$ where $\delta \omega=\left(\omega_{1}+\omega_{2}\right) / 2$. Unlike elastic order-parameter, $\varepsilon_{\text {inelastic }}(T)$ shown a peak at finite temperature $\left(T_{c}\right)$ and this can be understood as the difference between two elastic orderparameters, $I\left(Q_{0}, \Delta \omega_{0}, T\right)$ measure at $\Delta \omega_{0}=\omega_{1}$ and $\omega_{2}$. 


$$
\begin{gathered}
\varepsilon_{\text {inelastic }}(\delta \omega, T)=\int_{\omega_{1}}^{\omega_{2}} I\left(Q_{0}, \omega, T\right) d \omega \\
\varepsilon_{\text {inelastic }}(\delta \omega, T)=\int_{0}^{\omega_{2}} I\left(Q_{0}, \omega, T\right) d \omega-\int_{0}^{\omega_{1}} I\left(Q_{0}, \omega, T\right) d \omega
\end{gathered}
$$

where $\delta \omega=\left(\omega_{1}+\omega_{2}\right) / 2$. The $\varepsilon_{\text {inelastic }}(\delta \omega, T)$ is corresponding to a particular oscillation and the peak signal can be viewed as the resonance of the magnetic signal upon thermal fluctuations. Thus, On the other hand, we can think an elastic order-parameter as an integration of many infinitesimal inelastic order-parameters and the frozen temperature $T_{f}$ determined by elastic order-parameter at a given $\Delta \omega_{0}$ is the resonance temperature of fastest oscillation. Thus, the $T_{c}\left(\delta \omega_{0}\right)$ is approximately equal to the $T_{f}\left(\Delta \omega_{0}=\delta \omega_{0}\right)$. The inelastic order-parameters $\varepsilon_{\text {inelastic }}(\delta \omega, T)$ were calculated by integrating different energy bands of BASIS and DCS measurements and $\delta \omega_{0}$ - dependent $T_{c}\left(\delta \omega_{0}\right)$ was observed for all the three compounds (see Figure (App.) A.6 (b) for $C u \mathrm{Mn} 2 \%$, Figure (App.) A.5 (b) for $\operatorname{BCGO}(p=0.902(8))$ and Figure (App.) A.7 (b) for $\left.\mathrm{Y}_{2} \mathrm{Mo}_{2} \mathrm{O}_{7}\right)$.

The difference between two $I\left(Q_{0}, \Delta \omega_{0}, T\right)$ at $\Delta \omega_{0}=\delta \omega-\Delta$ and $\Delta \omega_{0}=\delta \omega+\Delta$, or $\varepsilon_{\text {inelastic }}(\delta \omega, T)$ directly measures the density of relaxation times within an interval defined by $[\delta \omega-\Delta, \delta \omega+\Delta]$. Thus, we could crudely map the distributions of relaxation time, $P(\tau)$ as a function of temperature for each system as shown in Figure 5.5 (b), (c) and (d). At temperatures well above the freezing temperature determined by the DC susceptibility $\left(T_{D C}\right), P(\tau)$ exhibits a distribution at shorter times $\left(\tau<10^{-3} \mathrm{~ns}=10^{-11} \mathrm{~s}\right)$. Upon cooling, $P(\tau)$ shows a sudden shift to longer times $\left(\tau>10^{12} \mathrm{~ns}=10^{3} \mathrm{~s}\right)$ and it is expected for archetypical spin glasses as a similar $P(\tau)$ behavior has been predicted for $C u \mathrm{Mn}$ by analyzing either bulk susceptibility [73] and Neutron scattering measurements [59]. The $P(\tau)$ at much higher temperature $\left(T \geq 10 T_{f}\right)$ in the paramagnetic regime (spin gas) is characterized by a nearly single Korringa relaxation time in the order of $10^{-12} \mathrm{~s}$. As $T$ is lowered, the correlations between spins starts to build and hence the collective motions among spins appear. This cause the Korringa like single relaxation time distribution to evolve into a much broader distribution at intermediate temperatures $\left(T_{f} \leq\right.$ $T<2.5 T_{f}$ ). Moreover, clustering of spins happens and cooperative relaxations among 
spins occurs, but the ergodicity of the system is not yet broken. Hence, this phase can be named as either co-operative paramagnet or spin liquid.

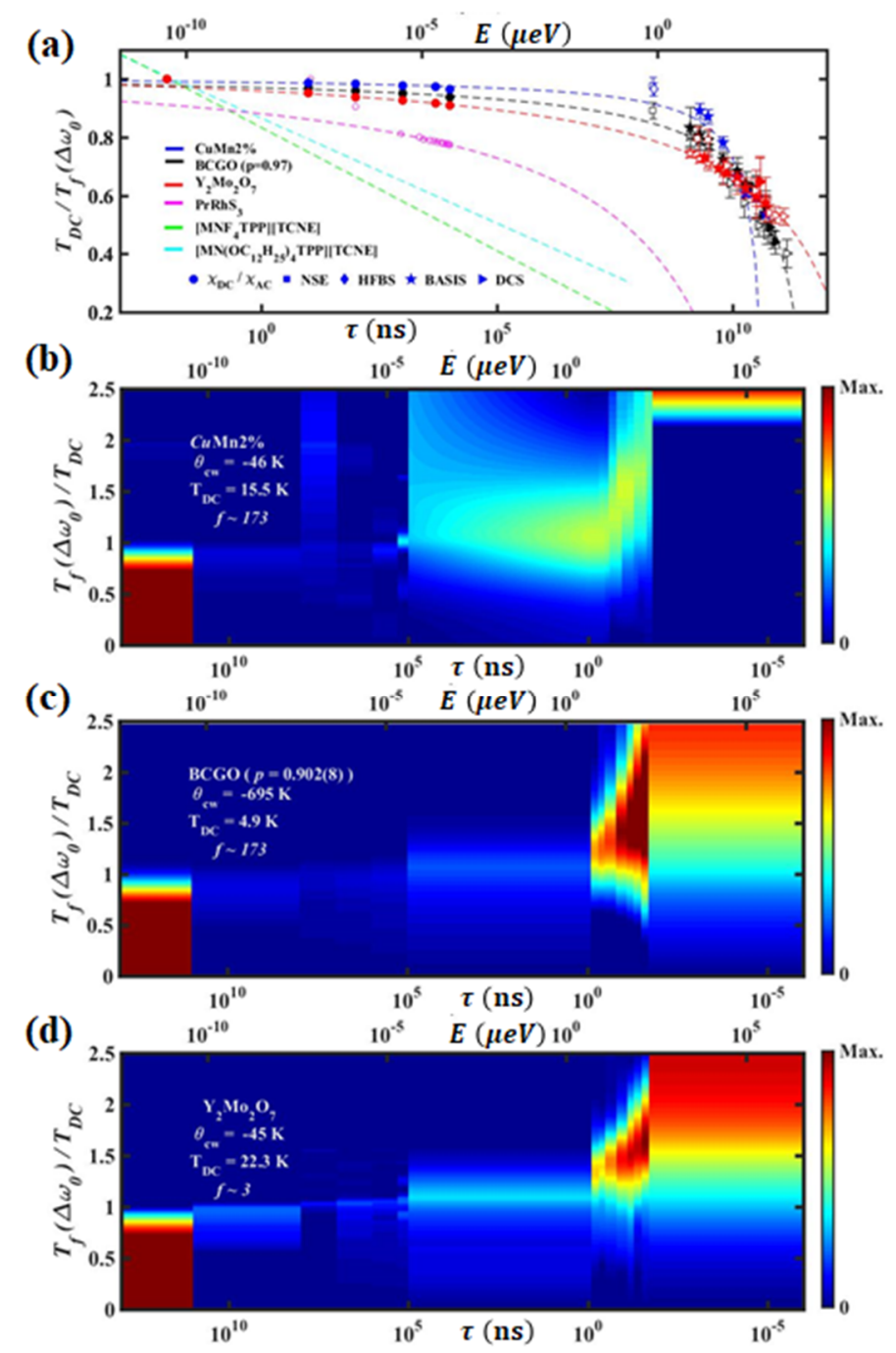

Figure 5.5 Freezing behaviors and relaxation time distributions of three magnetic glasses (a) summarizes the freezing temperature $T_{f}$, defined as the cusp of susceptibility (filled symbols) form elastic neutron scattering experiments as shown in Figure (App.) A.5, Figure (App.) A.6 and Figure (App.) $\quad$ A.7. The cups temperatures of neutron inelastic order parameter $T_{c}$ is also shown here as open symbols. The dash lines are the fittings of $T_{f}$ to the function $\frac{T_{f}(\tau)}{T_{0}}=\frac{A}{\log (\tau)+B}+$ $C$ derived from Vogal-Fulcher law. Here we have also included the freezing behaviors of three other magnetic systems: a ferromagnetic cluster spin-glass $\mathrm{PrRhSn}_{3}$ [74] and two molecular single chain magnet $\left[\mathrm{MnR}_{4} \mathrm{TPP}\right][\mathrm{TCNE}]$ and $\left[\mathrm{MN}\left(\mathrm{OC}_{12} \mathrm{H}_{25}\right)_{4} \mathrm{TPP}\right][\mathrm{TCNE}]$ which shows superparamagnetic behavior upon cooling [75]. The dashed line for $\operatorname{PrRhSn}_{3}$ is the fitting of $T_{f}$, obtained from ref. [74] to the aforementioned function while they are expected trends based on the AC susceptibility data from ref. [75] for the two superparamagnets [MnR 4 TPP][TCNE] and $\left[\mathrm{MN}\left(\mathrm{OC}_{12} \mathrm{H}_{25}\right)_{4} \mathrm{TPP}\right][\mathrm{TCNE}]$. 
Near glass transition $\left(T \approx T_{f}\right), P(\tau)$ become much broader and nearly flat. In further cooling $\left(T<T_{f}\right)$, spins will be frozen in time and will have much longer relaxations intrinsic to the topology of the energy landscapes of the magnetic glass system. From the Figure 5.5 (b), (c) and (d), we can only conclude that there are relaxations longer than $\tau_{0} \approx$ $100 \mathrm{~s}$ which is the longest $\tau_{0}$ experimentally possible to measure $I\left(Q_{0}, \Delta \omega_{0}, T\right)$. The study of relaxations below $T<T_{D C}$ in the magnetic glass state, is beyond the scope of this chapter. We will study longer relaxations by mean of aging and memory effects of different magnetic glasses in Chapter 6 and Chapter 7 .

Figure 5.4 (a) summarizes the $T_{D C} / T_{f}\left(\Delta \omega_{0}\right)$ in which $T_{f}\left(\Delta \omega_{0}\right)$ is estimated as the cusp of the susceptibility calculations. (see Figure (App.) A.6 (a) for $C u M n 2 \%$, Figure (App.) A.5 (a) for $\operatorname{BCGO}(p=0.902(8))$ and Figure (App.) A.7 (a) for $\left.\mathrm{Y}_{2} \mathrm{Mo}_{2} \mathrm{O}_{7}\right) . T_{f}\left(\Delta \omega_{0}\right)$ shows similar leveling out nature as a function of $\Delta \omega_{0}$, mimicking magnetic transitions in all the three systems, even though the overall freezing trends are quite different. For a more quantitative analysis, we fit $T_{D C} / T_{f}\left(\Delta \omega_{0}\right)$ to $\frac{T_{f}(\tau)}{T_{0}}=\frac{A}{\log (\tau)+B}+C$ and the fitting parameter are summarized in Table 5-1.

The fitting function is nothing other than the Vogel-Fulcher law, $\tau=\tau_{D} \exp \left[\frac{E_{a}}{k_{B}\left(T_{f}-T_{0}\right)}\right]$ for real glasses or supercooled liquids. Here $T_{0}$ can be understood as the phase transition from a liquid to a glass phase and $\tau_{D}$ is corresponding to the Debye frequency of the system and $E_{a}$ is the average activation energy of barriers in the energy landscape. In the limit of $T_{0} \rightarrow 0$, we can recover the Arrhenius law for thermal activation which is the typical behavior for Superparamagnets or cooperative paramagnets.

By rearranging the fitting function,

$$
\tau=e^{-B} e^{\frac{A}{\left(\frac{T_{f}}{T_{D C}}-C\right)}}
$$

Then, the fitting parameters can be realized by following relations,

$$
\tau_{D}=e^{-B}, \quad \frac{E_{a}}{k_{B}}=A T_{D C}, \quad T_{0}=C T_{D C}
$$


As summarized in Table 5-1, all the three systems seem to follow the Vogal-Fulcher law with reasonable values for $\tau_{D}, E_{a}$ and $T_{0}$, implying glass transitions.

Table 5-1 Fitting parameters of Vogal-Fulcher law for $\mathrm{BCGO}(p), \mathrm{Y}_{2} \mathrm{Mo}_{2} \mathrm{O}_{7}$ and $\mathrm{CuMn2 \%}$ The fitting parameters of Vogal-Fulcher law (Equation 5.6) and other relevant quantities (Equation 5.7) extracted from the frequency dependent ordering temperature of $\operatorname{BCGO}(p=0.902(8)), \mathrm{Y}_{2} \mathrm{Mo}_{2} \mathrm{O}_{7}$ and $C u \mathrm{Mn} 2 \%$, are summarized here.

\begin{tabular}{cccc}
\hline \hline Parameter & $\mathrm{BCGO}(p=0.902(8))$ & $\mathrm{Y} 2 \mathrm{Mo} 2 \mathrm{O} 7$ & $C u \mathrm{Mn} 2 \%$ \\
\hline $\boldsymbol{T}_{\mathbf{0}}$ & 4.94 & 22.35 & 15.04 \\
$\boldsymbol{A}$ & 1.4884 & 3.2533 & 1.1117 \\
$\boldsymbol{B}$ & 26.4 & 28.766 & 25.787 \\
$\boldsymbol{C}$ & 0.97465 & 0.93027 & 0.96389 \\
$\mathbf{1} / \boldsymbol{\tau}_{\boldsymbol{D}}=\boldsymbol{e}^{\boldsymbol{B}}$ & $2.9199 \mathrm{e}+11$ & $3.1111 \mathrm{e}+12$ & $1.5818 \mathrm{e}+11$ \\
$\boldsymbol{T}_{\boldsymbol{D}}=\frac{\mathbf{4}\left(\frac{\mathbf{1}}{\boldsymbol{t}_{\boldsymbol{D}}}\right) \mathbf{1 0}^{-\mathbf{1 2}}}{\boldsymbol{k}_{\boldsymbol{B}}}$ & 13.58 & 143.83 & 7.3 \\
$\boldsymbol{E}_{\boldsymbol{a}} / \boldsymbol{k}_{\boldsymbol{B}}=\boldsymbol{A} \boldsymbol{T}_{\boldsymbol{D C}}(\mathrm{K})$ & 7.35 & & 16.719 \\
$\boldsymbol{T}_{\mathbf{0}}=\boldsymbol{C} \boldsymbol{T}_{\boldsymbol{D C}}(\mathrm{K})$ & 4.79 & 20.79 & 14.44 \\
& & & \\
\hline
\end{tabular}

In the $T \rightarrow \infty$ limit, spin-spin correlations of a magnetic system are limited only to on-site correlations and the corresponding relaxations are characterized by Korringa relaxation time which is analogous to Debye frequency of atomic crystals. With lowering temperature, spins will pair up into clusters by increasing the density of longer relaxation times at temperature above glass transitions $\left(T>T_{0}\right)$. Below transition temperature $T \leq$ $T_{f}$, a spin glass system will freeze into an infinite cluster comprised with randomly oriented smaller clusters. Thus, the $\tau-$ dependence of $T_{f}$ tells how the clustering happens in the cooperative paramagnetic regime. Moreover, we can find a critical relaxation time $\left(\tau_{c}\right)$ where $T_{f}$ starts to level off, for each magnetic glass and it is corresponding to the biggest locally ordered cluster formed upon cooling. Notice that the $\tau_{c}$ is a function of parameter $A$. Apparently, the parameter $A$ for $\operatorname{BCGO}(p=0.902(8))$ and $\mathrm{Y}_{2} \mathrm{Mo}_{2} \mathrm{O}_{7}$ is higher comparing to the archetypical spin glass $C u \mathrm{Mn} 2 \%$; so the $\tau_{c}$. This is a direct evidence of having frozen states comprised with much bigger locally ordered clusters in the case of $\operatorname{BCGO}(p=$ 
$0.902(8)$ ) and $\mathrm{Y}_{2} \mathrm{Mo}_{2} \mathrm{O}_{7}$. This behavior also agrees with the finite correlation lengths at a non-zero wave-vector observed in neutron scattering experiments for $\mathrm{BCGO}(p=0.902(8))$ and $\mathrm{Y}_{2} \mathrm{Mo}_{2} \mathrm{O}_{7}$ magnets.

Figure 5.5 (a) summarizes three other magnetic systems from literature for a broader view of clustering in the magnetic material. Those are a ferromagnetic cluster spin-glass $\mathrm{PrRhSn}_{3}$ [74] and two molecular single-chain magnet [MnR 4 TPP][TCNE] and $\left[\mathrm{MN}\left(\mathrm{OC}_{12} \mathrm{H}_{25}\right)_{4} \mathrm{TPP}\right][\mathrm{TCNE}]$ which shows superparamagnetic behavior upon cooling [75]. The $T_{f}$ obtain from the ref. [74] was fitted to the Vogal-Fulcher law and the parameter $A$ turns out to be $\sim 5$ for the cluster-glass, $\mathrm{PrRhSn}_{3}$. This value is much larger than the previously observed values from magnetic glasses, and it is understandable that the frozen state of a cluster glass is formed by bigger clusters than usual magnetic glasses. For $\left[\mathrm{MnR}_{4} \mathrm{TPP}\right][\mathrm{TCNE}]$ and $\left[\mathrm{MN}\left(\mathrm{OC}_{12} \mathrm{H}_{25}\right)_{4} \mathrm{TPP}\right][\mathrm{TCNE}]$ magnets, we are plotting possible trends based on the AC susceptibility measurement from ref. [75]. Even in a Superparamagnets, the clustering happens upon cooling, but it will never freeze into an $\infty$-cluster like in magnetic glasses instead remain as a pool of clusters in different sizes down to near absolute zero. 


\subsection{Glassy relaxation under field}

We performed bulk susceptibility experiments as a function of magnetic field on the three magnetic glasses, $C u \mathrm{Mn} 2 \%, \operatorname{SCGO}(p=0.968(6))$ and $\mathrm{Y}_{2} \mathrm{Mo}_{2} \mathrm{O}_{7}$. First, we cooled down a sample to the base temperature $\left(T_{\text {base }}\right)$ of the instrument $(T \sim 2 \mathrm{~K})$ under zero-filed condition and applied a magnetic field $(H)$ and measure the DC susceptibility $\left(\chi_{D C}\right)$ while heating. Once the sample reached a temperature well above the magnetic transition $\left(T \sim 2 T_{f}\right)$, it was cooled down again to $T_{\text {base }}$ and measuring $\chi_{D C}$ while cooling. Thus, we repeated this sequence for several field values. Whenever non-ergodic magnetic phase appears in a magnetic system, we can typically detect that by a bifurcation of zero-field cooled (ZFC) and field-cooled (FC) susceptibilities. During this experiment, we monitor the irreversible temperature $T_{i r r}$ defined as the temperature where the bifurcation starts, as a function of $H$.

In a magnetic glass, the ergodicity is broken below the glass transition temperature due to the ruggedness of the corresponding complex energy landscapes. Hence the system will trap in a local-minima in which the net magnetization is zero under zero-field conditions, by restricting the entire phase space other than the entropically available states around that minima. Under an external magnetic field, the energy landscape will be modified by the Zeeman term $\left(-H . \sum S_{i}\right)$ such a way that the configuration favors the magnetic field ( $\sum \hat{n} . S_{i}>0 ; \hat{n}$ - the direction of the magnetic field) are lower in energy and higher in magnetization. In the zero-field cooling case, we apply the magnetic field while the system is in thermal equilibrium at $T_{\text {base }}$. The occupation probability of unfavored configurations versus favored configurations are equal under zero-field cooling. Thus, the unfavored configurations are entropically trapped at low temperature for an applied field low enough to overcome nearby barriers by causing lower magnetization below $T_{f}$. But, in the case of field-cooling, the system populates mostly the configurations favored by the magnetic field by causing a higher magnetization comparing to zero-field cooling. Thus, the thermal hysteresis of ZFC-FC in DC-susceptibility is a direct evidence of ergodicity breaking in the time scale of $\sim 100 \mathrm{~s}$. 

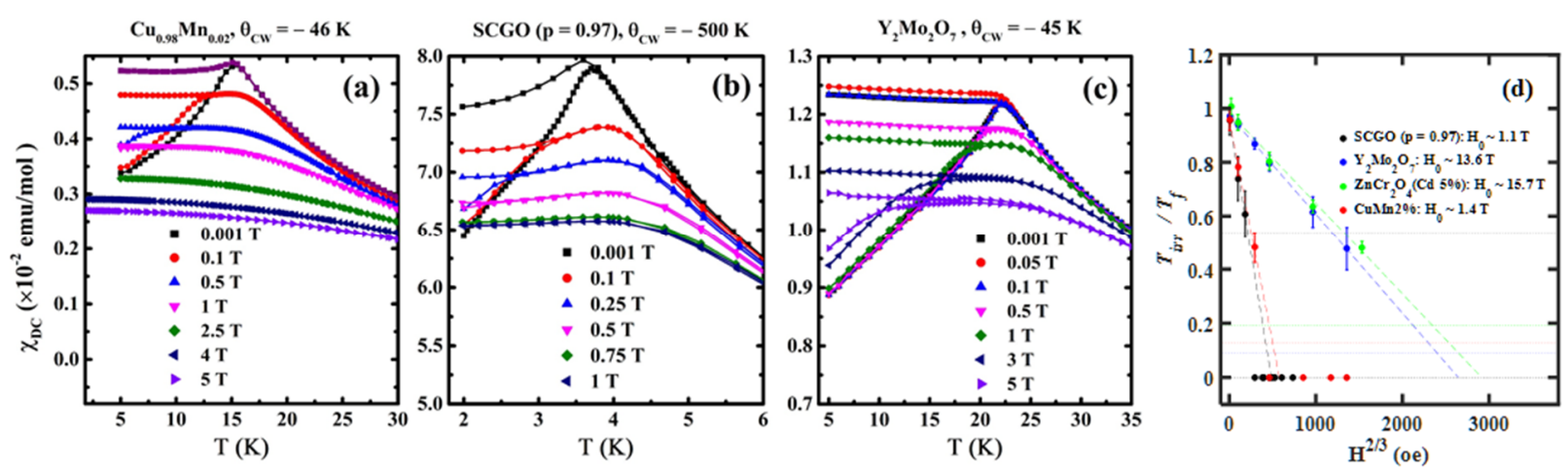

Figure 5.6 ZFC-FC as a function of field

The ZFC-FC magnetization measurements as a function of magnetic field for (a) $\operatorname{SCGO}(p=0.96$ ), (b) Y2Mo2O7 and (c) $\mathrm{CuMn} 2 \%$.

As shown in Figure 5.6 (a), (b) and (c), All the magnetic glasses show the ZFC-FC bifurcation below $T_{D C}$ and the $T_{i r r}$ decreases with increasing field. Above a critical field $\left(H_{0}\right)$, the bifurcation will be completely closed, and the ground state will be a smooth energy landscape with a single minimum. Depending on the distribution of potential barriers in the ground state, different magnetic systems show different behaviors over magnetic field. For a magnetic glass, a linear behavior of $T_{i r r}$ with $H^{2 / 3}$ is expected and

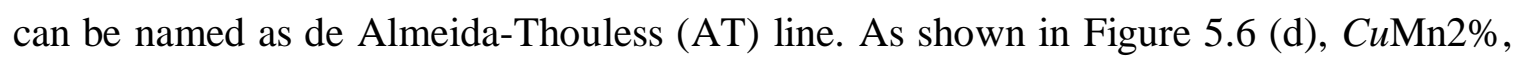
$\operatorname{SCGO}(p=0.968(6))$ and $\mathrm{Y}_{2} \mathrm{Mo}_{2} \mathrm{O}_{7}$ show the expected linear dependence of $T_{i r r}$ as a function of $H^{2 / 3}$ by confirming the low temperature phases of $\operatorname{BCGO}(p=0.902(9))$ and $\mathrm{Y}_{2} \mathrm{Mo}_{2} \mathrm{O}_{7}$ are glass-like.

Table 5-2 Comparison of critical field extracted from ZFC-FC magnetization experiments The critical fields $\left(H_{0}\right)$ of $\mathrm{SCGO}(p=0.97), \mathrm{Y}_{2} \mathrm{Mo}_{2} \mathrm{O}_{7}$ and $C u \mathrm{Mn} 2 \%$ compounds are estimated by the extrapolating $T_{i r r}$ versus $H$ data as shown in the Figure 5.6 (d) and the corresponding magnetic energies $\left(E_{B}\right)$ are also calculated as summarized here.

\begin{tabular}{cccc}
\hline \hline Parameter & $\mathrm{SCGO}(p=0.97)$ & $\mathrm{Y} 2 \mathrm{Mo} 2 \mathrm{O} 7$ & $\mathrm{CuMn} 2 \%$ \\
\hline $\boldsymbol{S}$ & $3 / 2$ & 1 & $3 / 2$ \\
$\boldsymbol{H}_{\mathbf{0}}(\mathrm{T})$ & 2 & 20.6 & 15.1 \\
$\boldsymbol{E}_{\boldsymbol{B}} / \boldsymbol{k}_{\boldsymbol{B}}=\frac{\boldsymbol{\mu}_{\boldsymbol{B}} \boldsymbol{g} \boldsymbol{S H}_{\mathbf{0}}}{\boldsymbol{k}_{\boldsymbol{B}}}(\mathrm{K})$ & 4 & 27.6 & 4.8 \\
$\boldsymbol{T}_{\boldsymbol{f}}(\mathrm{K})$ & 3.8 & 22.5 & 15.5 \\
$\boldsymbol{\theta}_{\boldsymbol{c} \boldsymbol{w}}(\mathrm{K})$ & -500 & -45 & -46 \\
$\boldsymbol{E}_{\boldsymbol{B}} / \boldsymbol{k}_{\boldsymbol{B}} \boldsymbol{T}_{\boldsymbol{f}}$ & $\mathbf{1 . 0 5}$ & $\mathbf{1 . 2 3}$ & $\mathbf{0 . 3 1}$ \\
\hline
\end{tabular}


The critical field, $H_{0}$ of a particular magnetic glass can be estimated by the intercept of the linear fit and the corresponding $H_{0}$ of the three magnetic glasses are summarized in the Table 5-2. The magnetic energy $\left(E_{B}\right)$ corresponding to $H_{0}$ represents the required energy to completely remove the ruggedness of the energy landscape. Notice that in the case of $\operatorname{SCGO}(p=0.968(6))$ and $\mathrm{Y}_{2} \mathrm{Mo}_{2} \mathrm{O}_{7}$, the $E_{B} / k_{B}$ is comparable with $T_{f}$, while $E_{B} / k_{B}$ is about three times lower comparing to $T_{f}$. (see the $E_{B} / k_{B} T_{f}$ ratio in Table 5-2) This might be an indication of $\operatorname{SCGO}(p=0.968(6))$ and $\mathrm{Y}_{2} \mathrm{Mo}_{2} \mathrm{O}_{7}$ magnets belong to a different category of magnetic glasses, distinct from dilute spin glasses.

\subsection{Conclusions}

Three different magnetic glasses systems: a dilute magnetic alloy $C u \mathrm{Mn} 2 \%$, a quasi-2D frustrated magnet $\operatorname{SCGO}(p=0.968(6)) / \operatorname{BCGO}(p=0.902(8))$ and a 3D pyrochlore magnet $\mathrm{Y}_{2} \mathrm{Mo}_{2} \mathrm{O}_{7}$ has been revived and compared using different experimental techniques. All the three systems show glass transitions below a finite temperature much lower than the corresponding Curie-Weiss temperature. But the magnetic glass states from the dense limit of geomerically frustrated magnets mentioned above deviate from the conventional spin glass behavior observed in $C u \mathrm{Mn} 2 \%$ implying that the magnetic glasses needs more refined categorization. 


\section{Chapter 6}

\section{Aging, Memory, and Nonhierarchical energy landscape of SCGO}

\subsection{Motivation}

If the energy landscape of a system resembles a smooth vase with a pointy bottom end, upon cooling the system goes quickly into the lowest energy state, i.e., the global ground state that is usually associated with the crystalline order. If the energy landscape is more complex with many metastable states, i.e., local minima, then cooling may lead the system into local minima resulting in a glassy order. The concept of such energy landscapes has been instrumental in explaining the glassiness that is ubiquitous in a wide range of systems, including atomic clusters [76], structural glasses [77,78], polymers [79], brain activity [80], and social networks [81]. Several different topological types of energy landscapes were proposed to characterize different glassiness and the associated slow dynamics $[82,83]$. For instance, a rugged funnel-shaped landscape shown in Figure 6.1(a) was proposed to understand the physics of biopolymers [84,85] and dilute magnetic alloys called spin glass [7].

Magnetic glass systems [86--90] present a unique opportunity to microscopically study the relation between the energy landscape and low-temperature properties. The most studied magnetic glass state is the conventional spin glass realized in dilute magnetic alloys such as $C u \mathrm{Mn}$ and $A u \mathrm{Fe}$. Here, dilute magnetic ions (Mn and Fe) in a nonmagnetic metal interact via the long-range Ruderman-Kittel-Kasuya-Yosida (RKKY) interaction whose magnitude and sign change with distance between the randomly placed magnetic ions [7]. 
(a)

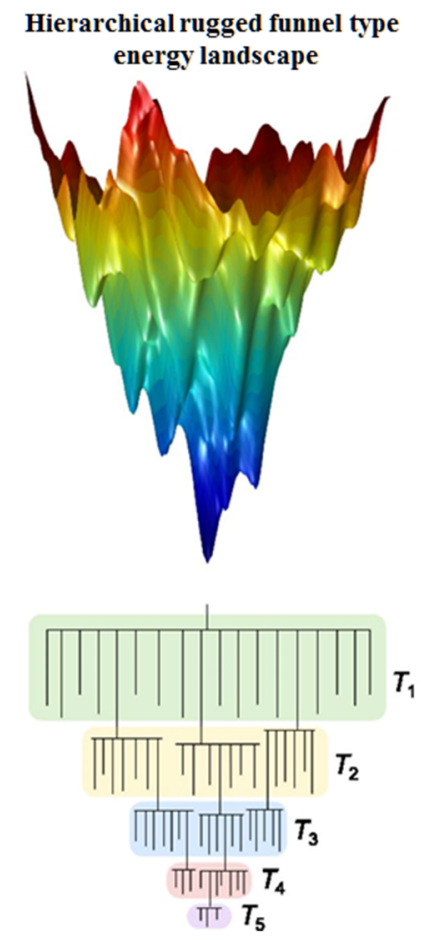

(b)

$$
\begin{gathered}
\text { Non-hierarchical energy landscape with } \\
\text { a wide Nearly flat rough bottom }
\end{gathered}
$$
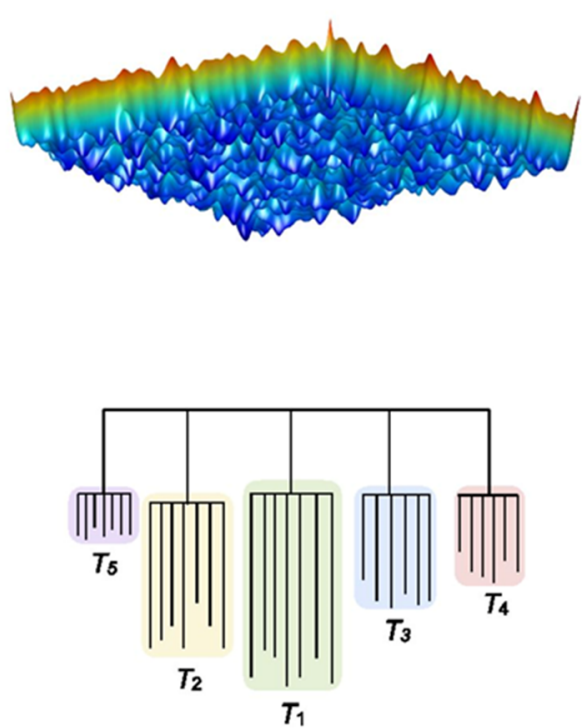

Figure 6.1 Schematic energy landscapes of spin glass and spin jam.

(a) (Upper) Schematic energy landscape of a conventional spin glass that consists of many hierarchical rugged funnels and (Lower) the corresponding hierarchical tree representation. (b) (Upper) Schematic diagram of nonhierarchical energy landscape of the spin jam that has wide nearly flat rough bottom and (Lower) the corresponding nonhierarchical barrier tree representation.

The randomness drives the system into the spin glass state below a critical temperature, $T_{f}$ , that is comparable to the mean-field magnetic energy scale, i.e., the absolute value of the Curie-Weiss temperature, $\left|\Theta_{C W}\right|$. For instance, for $\mathrm{Cu}-2$ at. \% $\mathrm{Mn}(C u \mathrm{Mn} 2 \%$ hereafter $)$, $\Theta_{C W}=-45 \mathrm{~K}$ and $T_{f}=15.5 \mathrm{~K}$. Another distinct glassy state called a spin jam has been recently suggested to appear in densely populated frustrated magnets [4,5,34,35,72]. At the mean-field level, these systems are expected to remain in a classical spin liquid down to absolute zero temperature, due to macroscopic classical ground state degeneracy. Quantum fluctuations, however, lift the degeneracy and lead the system to the spin jam state below $T_{f}$ that is much lower than $\left|\Theta_{C W}\right|$. For instance, for $\operatorname{SrCr}_{p p} \mathrm{Ga}_{12-9 p} \mathrm{O}_{19}(\mathrm{SCGO})(p$ $=0.97), \Theta_{C W}=-500 \mathrm{~K}$ and $T_{f}=3.8 \mathrm{~K}$. The stark different ratios of $\left|\Theta_{C W}\right| / T_{f}$ for the 
spin jam and spin glass suggest that the two states might have qualitatively distinct energy landscapes.

\subsection{Aging and Memory Effect Experiments}

Aging and memory effects have been key features of glassy systems due to the intrinsic slow-dynamics. The thermo-remanent magnetization (TRM) method is the most effective way so far to investigate these effects $[88,89,91]$; for the measurements, the sample is first cooled down from well above $T_{f}$ to base temperature with a single stop for a waiting time, $t_{w}$, at an intermediate temperature $T_{w}$, under zero field. While waiting at $T_{w}$, if the system has as many nearly degenerate metastable states at low energies as spin jam and conventional spin glasses have, the system will relax to the accessible lower energy states than when no waiting is imposed. The longer $t_{w}$ is, the lower energy states the system will relax to, which is called "aging". Once cooled down to base temperature, the TRM is measured by applying a small field of a few gauss upon heating at a constant rate. During the measurements, when the temperature approaches the temperature of aging, $T_{w}$, the system revisits the lower energy states reached during the wait time that are associated with the energy scale of $k_{B} T_{f}$, where $k_{B}$ is the Boltzmann constant. Upon further heating, the system goes to higher energy states allowed within $k_{B} T$. This is referred to as the aging and memory effect.

\subsection{Magnetic Memory of Spin Jam vs Spin Glass}

We have performed the TRM measurements on two spin jam prototypes, $\operatorname{SCGO}(p=0.97)$ and $\mathrm{BaCr}_{9 p} \mathrm{Ga}_{12-9 p} \mathrm{O}_{19}(\mathrm{BCGO})(p=0.96)$ in which the magnetic $\mathrm{Cr}^{3+}\left(3 \mathrm{~d}^{3}\right)$ ions form a highly frustrating quasi-2D triangular network of bipyramids [4,5,34,35,72] and a spin glass prototype $\mathrm{CuMn} 2 \%$ in which the $2 \%$ low concentration of the magnetic $\mathrm{Mn}$ atoms is embedded in the nonmagnetic $\mathrm{Cu}$ metal. Strong aging and memory effects have been observed in $C u \mathrm{Mn} 2 \%$, whereas the effects are much weaker in SCGO and BCGO. Figure 6.2 (a)-(c) shows the TRM data obtained from $\operatorname{SCGO}(p=0.97), \operatorname{BCGO}(p=0.96)$, and $C u \mathrm{Mn} 2 \%$, respectively, with several different values of $t_{w}$ ranging from 6 min to $100 \mathrm{~h}$, at $T_{w}=T_{f} \sim 0.7$. All samples exhibit similar aging and memory effects that increase with increasing $t_{w}$. These indicate the existence of numerous metastable states and slow 
dynamics in all systems. Despite the similarity, there is a clear difference: For the $\mathrm{CuMn} 2 \%$ magnetic alloy, considerable aging occurs at $T_{w}$ even for a short $t_{w}$ of 6 min (data in violet in Figure 6.2(c)), whereas for the spin $\operatorname{jam} \operatorname{SCGO}(p=0.97)$ and $\operatorname{BCGO}(p=0.96)$, there is very small aging for $t_{w}=6 \mathrm{~min}$ (data in violet in Fig. $2 \mathrm{~A}$ and B). Furthermore, in the case of $\mathrm{CuMn} 2 \%$, as $t_{w}$ increases, the memory effect increases to develop a dip at $T_{w}$ for $t_{w} \geq$ $3 \mathrm{~h}$. On the other hand, for $\operatorname{SCGO}(p=0.97)$ such a dip never appears even for $t_{w}=100 \mathrm{~h}$; instead only a weak memory shoulder appears.
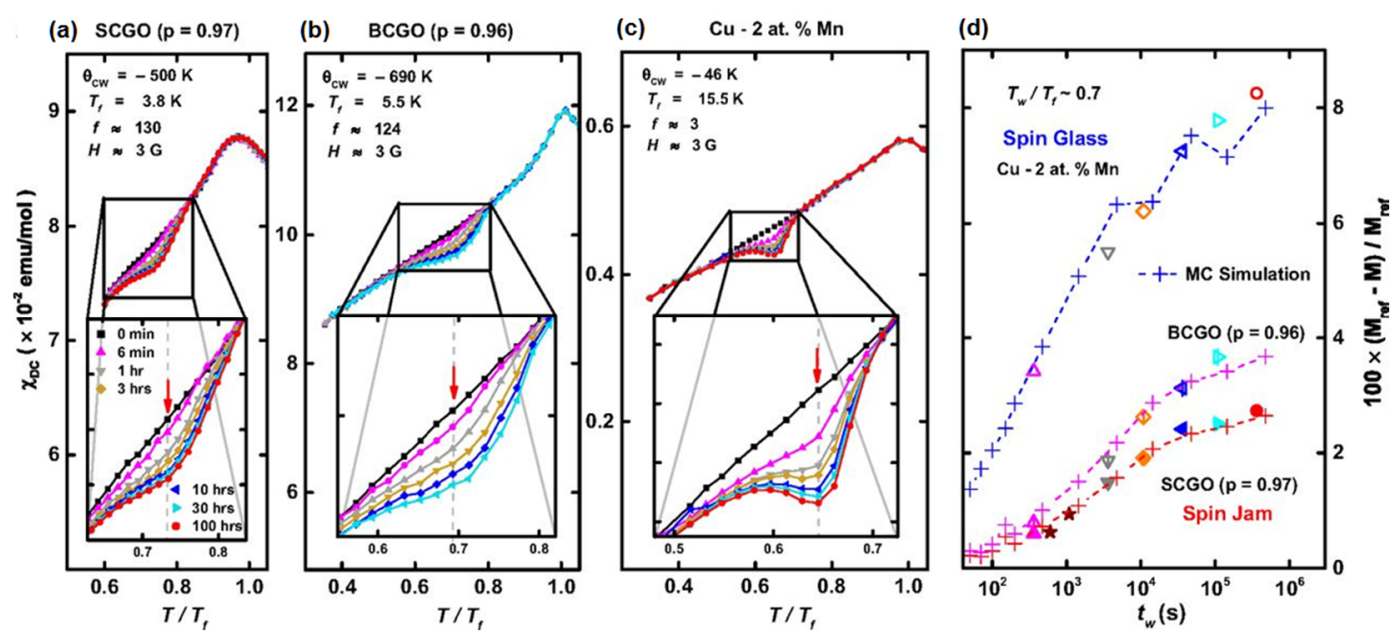

Figure 6.2 Magnetic memory as a function of $\boldsymbol{t}_{w}$ for SCGO, BCGO and $\mathrm{CuMn} 2 \%$.

(a)-(b) Bulk susceptibility, $\chi_{D C}=M / H$, where $M$ and $H$ are magnetization and applied magnetic field, respectively, obtained from (a) $\operatorname{SCGO}(p=0.97)$, (b) $\operatorname{BCGO}(p=0.96)$, and (c) a spin glass $\mathrm{CuMn} 2 \%$, with $H=3 \mathrm{G}$. Symbols and lines with different colors indicate the data taken with different waiting times, $t_{w}$, ranging from $0 \mathrm{~h}$ to $100 \mathrm{~h}$, at $T_{w} / T_{f} \sim 0.7$, where $T_{w}$ and $T_{f}$ are the waiting and the freezing temperature, respectively. (d) From the data shown in (a)-(c), the aging effect was quantified for the three systems by $\left(M_{r e f}-M\right) / M_{r e f}$, where $M_{r e f}$ is the magnetization without waiting, and it was plotted as a function of $t_{w}$ in a log scale. The "+" symbols mark the results of our MC simulations. Details of the simulations can be found in Appendix C .

The memory effect can be quantified by the aging-induced relative change in the magnetization, $\left(M_{\text {aging }}-M_{\text {ref }}\right) / M_{\text {ref }}$, where $M_{\text {aging }}$ and $M_{\text {ref }}$ are the magnetization with and without aging, respectively. Figure 6.2(d) shows $\left(M_{\text {aging }}-M_{\text {ref }}\right) / M_{\text {ref }}$, measured at $T_{w} / T_{f} \sim 0.7$ for $\operatorname{SCGO}(p=0.97$ ) (solid symbols), $\operatorname{BCGO}(p=0.96)$ (symbols with a line), and CuMn2\% (open symbols), as a function of $t_{w}$. These data are consistent with a previous study on $\operatorname{SCGO}(p=0.956)$ with $t_{w}$ up to $5.83 \mathrm{~h}(13)$. In the case of $C u \mathrm{Mn} 2 \%$, as $t_{w}$ increases from $6 \mathrm{~min}$ to $100 \mathrm{~h},\left(M_{\text {aging }}-M_{\text {ref }}\right) / M_{\text {ref }}$ continues to 
gradually increase from $3.4 \%$ to $8.2 \%$. On the other hand, for $\operatorname{SCGO}(p=0.97)$, $\left(M_{\text {aging }}-M_{\text {ref }}\right) / M_{\text {ref }}$ increases gradually from $0.6 \%$ to $2.4 \%$, and for $\operatorname{BCGO}(p=0.96)$, from $0.7 \%$ to $3.1 \%$, as $t_{w}$ increases from $6 \mathrm{~min}$ to $10 \mathrm{~h}$. The increase rate of $\left(M_{\text {aging }}-M_{\text {ref }}\right) / M_{\text {ref }}$ seems to decrease for $t_{w}>10 \mathrm{~h}$, reaching $2.7 \%$ at $t_{w}=100 \mathrm{~h}$ for $\operatorname{SCGO}(p=0.97)$. We emphasize that over this wide range of $t_{w}$ up to $100 \mathrm{~h}$ the susceptibility curve is always monotonically dependent on temperature up to the freezing point (Figure 6.2 (a) and (b)), in sharp contrast to $C u \mathrm{Mn} 2 \%$.

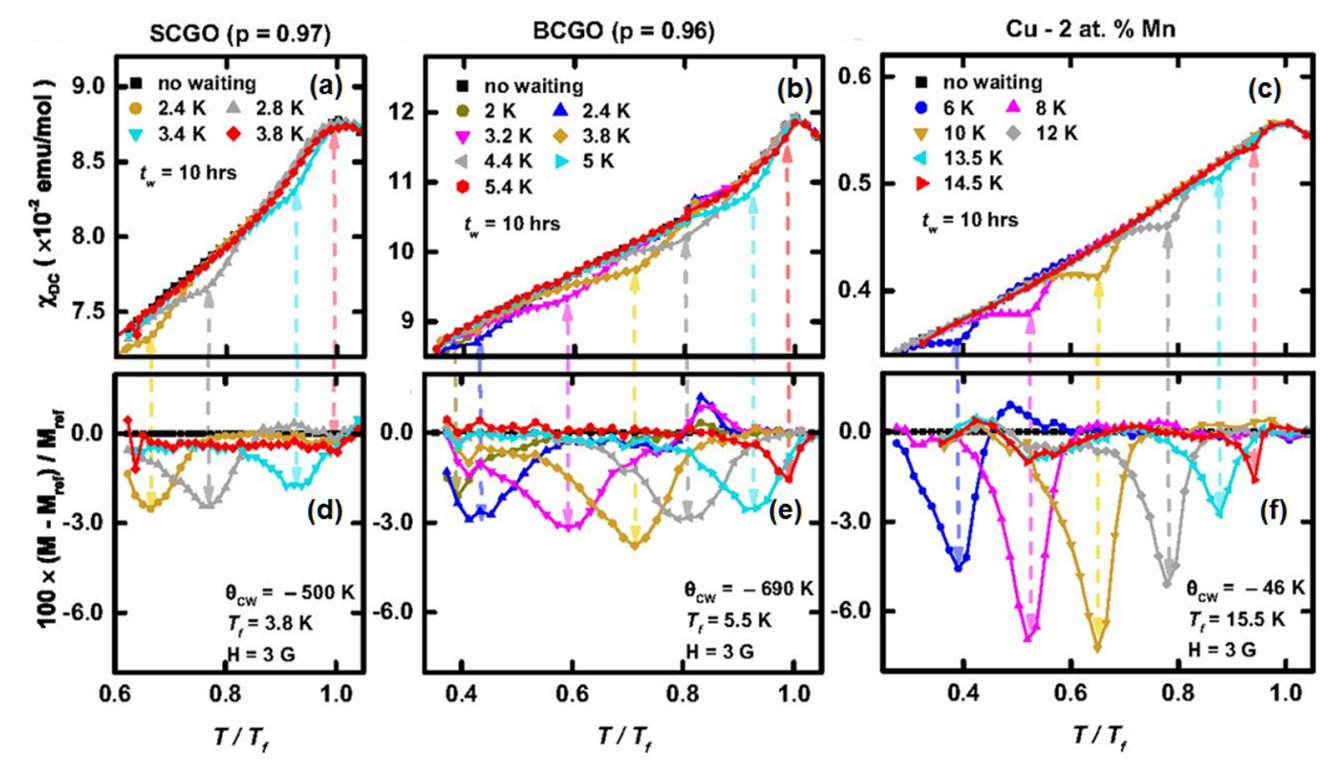

Figure 6.3 Magnetic memory as a function of $\boldsymbol{T}_{w}$ for SCGO, BCGO and $\boldsymbol{C u M n 2 \%}$.

(a)-(c) $\chi_{D C}$ and (d)-(f) $\left(M_{r e f}-M\right) / M_{\text {ref }}$ measured for [(a) and (d)] $\operatorname{SCGO}(p=0.97)$, [(b) and

(e) $\operatorname{BCGO}(p=0.96)$, and [(c) and (f)] $C u \mathrm{Mn} 2 \%$, with $t_{w}=10 \mathrm{~h}$, at various waiting temperatures.

Figure 6.3 shows the memory effect for various values of $0.4 \lesssim T_{w} / T_{f} \lesssim 1$ measured with $t_{w}=10 \mathrm{~h}$. All systems exhibit maximal memory effect for $T_{w} / T_{f} \sim 0.7$. When $T_{w} / T_{f}$ increases or decreases from the maximal value, then the memory effect becomes weaker. The weakening, however, is more rapid in $C u \mathrm{Mn} 2 \%$ than in SCGO and BCGO; for $C u \mathrm{Mn} 2 \%,\left(M_{\text {aging }}-M_{\text {ref }}\right) / M_{\text {ref }}$ decreases from $7.2 \%$ for $T_{w} / T_{f} \sim 0.7$ to $2.7 \%$ for $T_{w} / T_{f}$ $\sim 0.9$, whereas for $\operatorname{SCGO}(p=0.97)[\operatorname{BCGO}(p=0.96)],\left(M_{\text {aging }}-M_{\text {ref }}\right) / M_{\text {ref }}$ decreases from $2.4 \%(3.1 \%)$ for $T_{w} / T_{f} \sim 0.7$ to $1.7 \%(2.5 \%)$ for $T_{w} / T_{f} \sim 0.9$. The pronounced memory effects found in CuMn2\% may hint at an energy landscape with a more hierarchical structure. On the other hand, the weak memory effect, observed in SCGO and 
BCGO, which is uniform over a wide range of $0.4 \lesssim T_{w} / T_{f} \lesssim 1$, suggests an energy landscape with a less hierarchical structure.

\subsection{Dynamical Tree Model Simulations}

Rejuvenation and memory effects have proved difficult to reproduce in standard simulations of supercooled liquids or spin glasses, due to the large phase space to be covered and a large spread of time scales involved [92,93]. Several successful attempts were made, such as a multilayer random energy model [94] and a model of thermally activated number sorting [93]. None of the studies, however, investigated how different topologies of the energy landscape will impact the memory effects. Here we have done so by taking a phenomenological approach based on a multilayer energy model. As shown later, this approach reproduces qualitatively the differences between memory effects associated with different landscapes.

We performed Monte Carlo simulations on two types of energy landscapes suggested for the spin glass and spin jam. Although the energy surface in both cases is characterized by numerous local minima, the distribution and connectivity of these minima are very different. Here we adopt the so-called barrier tree representation $[83,95,96]$ in which the local minima correspond to leaves of the tree, whereas the branching points denote the barriers separating disconnected valleys and/or minima. Details can be found in Figure (App.) C.1 and discussion in Appendix C .

Figure 6.1(a) shows a funnel-type barrier tree that is characteristic of the conventional spin glass. A rugged funnel here corresponds to a single long branch (the global minimum) with many dead branches splitting from it $[83,95]$. The experimentally observed memory effect is intimately related to a multitude of energy and time scales in the low-energy configurational space. For the funnel-type landscape, a hierarchical structure of energy scales is encoded in the different levels of the barrier tree. The energy barriers $\varepsilon_{l}$ at level $l$ are characterized by a temperature $T_{l}$ such that $T_{1}>T_{2}>\cdots>T_{L}$, where $L$ is the number of levels of the tree [94]. The freezing temperature is $T_{f} \approx T_{1}$. The relaxation of the system in this hierarchical structure exhibits complex temperature- dependent dynamics. Typically, because the relaxation time at level $l$ scales as $\tau_{l} \sim \tau_{0} e^{\varepsilon_{l} / T}$, where $\tau_{0}$ is a 
microscopic time scale, the relaxation dynamics start to show exponential slowing down at level $l$ when $T<T_{l}$. Depending on the population of dead-end local minima at each level, the system fluctuates over a small window of levels determined by $T_{w}$ in the experiments. A longer $t_{w}$ at this temperature allows the system to relax to a deeper and larger (entropically) valley of the energy surface. The memory effect observed during the reheating process results from the fact that the system is trapped in this special landscape basin. The susceptibility, $\chi_{D C}$, as a function of temperature is shown in Figure 6.4 (a)-(c) for three different $T_{w}$. The DC susceptibility computed using a random magnetization model [94] shows a clear dip that depends on $T_{w}$ as well as $t_{w}$. In particular, a longer $t_{w}$ gives rise to a larger susceptibility reduction. It should be noted that other contributions to $\chi_{D C}$ such as continuous spin fluctuations are not included in the landscape tree dynamics simulations.
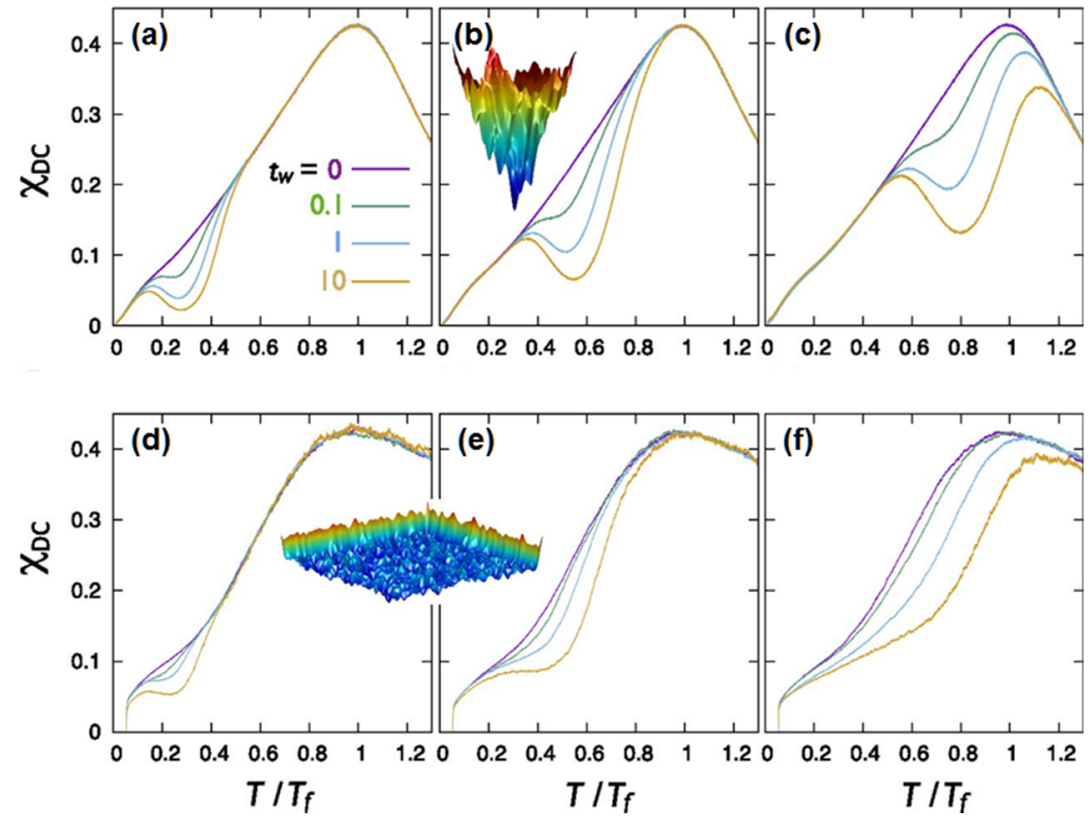

Figure 6.4 The simulated DC susceptibility.

(a)-(c) and (d)-(f) show the simulated DC susceptibility during the reheating process for the hierarchical and nonhierarchical trees, respectively, at three different waiting temperatures $T_{w}=$ $0.2 T_{f}\left((\mathrm{a})\right.$ and (d)), $0.4 T_{f}$ ((b) and (e)), and 0.6T $[(\mathrm{c})$ and (f)]. Different curves in each panel correspond to varying $t_{w}$ measured in units of the total cooling time.

In contrast to ordinary spin glass, the energy structure in a spin jam results from quantum and classical fluctuations breaking an exactly flat landscape [5]. Importantly, the energy 
scale for glass transition $T_{f}$ is determined by the fluctuations and is two orders of magnitude smaller than the Curie-Weiss temperature [5]. We expect the resulting landscape to feature broad basins and within each basin numerous microstates, as shown in Figure 6.1(b). As the local minima in spin jam result from the original zero energy mode of the classical spin Hamiltonian, it is plausible that the energy minima here are clustered into different branches (labeled by $m$ ) each characterized by a different energy scale $T_{m}$. For a particular cluster or branch of minima, the temperature $T_{m}$ underscores the energy barrier due to quantum fluctuations. $T_{m}$ is a random variable and is uniformly distributed in the interval of $\left[0, T_{f}\right] . T_{w}$ sets a threshold such that clusters with $T_{m}>T_{w}$ exhibit slow relaxation dynamics, whereas a longer $t_{w}$ helps the system find the cluster with a lower overall energy and larger entropy. This property underscores the weak memory effect observed in spin jam. Again, the fact that the system is trapped in this special cluster manifests itself as the memory effect during rewarming. The simulated susceptibility of the nonhierarchical tree, shown in Figure 6.4 (d)-(f), shows a memory effect that depends on both $T_{w}$ and $t_{w}$, similar to the spin glass. However, the salient feature is rather different: Contrary to the narrow dip in the hierarchical tree that appears even for short waiting time $t_{w}>1$ (Figure 6.4 (a)(c)), the susceptibility here exhibits a wide shoulder-like feature over a much wider range of $t_{w}$ for each $T_{w}$. As shown in more detail in Figure (App.) C.2 (a), for $T_{w}=0.6 T_{f}$ the nonhierarchical landscape fails to yield a narrow dip over six orders of magnitude of the Monte Carlo (MC) steps. Remarkably, this finding is consistent with the experimental data revealing a shoulder-like feature for spin jam (Figure 6.2 (a) and (b)) vs. the substantial dip for the ordinary spin glass (Figure 6.2 (c)). Furthermore, the functional dependence of the memory effect on waiting time is nicely reproduced for both systems in Figure 6.2 (d) over three orders of magnitude.

\subsection{Discussion}

The picture that emerges from the bulk susceptibility and Monte Carlo simulations is that the energy landscape of a spin jam is qualitatively different from the rugged funnel-type landscape of a spin glass. The hierarchical structure allows a natural realization of multiple energy scales (e.g., ref. [97]) that is crucial to the memory effect. On the other hand, the aging dynamics in the spin jam are well described by an essentially nonhierarchical barrier 
tree with more uniform branching. This result is consistent with the fact that the rough energy landscape in spin jam results from quantum fluctuations that lift the otherwise degenerate classical ground states. In particular, the weak memory effect at short times found in a spin jam may be interpreted as a result of the large time it takes the system to wander among the numerous roughly equivalent minima at a given energy scale.

The transition from a spin liquid to a spin jam in densely populated frustrated magnets upon cooling may be viewed as an effective reduction of degrees of freedom. In SCGO [4,5] and kagome antiferromagnet [15] the origin of the reduction can be induced by quantum fluctuations. We remark that the transition bears some analogy to the transition from a structural (mechanical) liquid state to a mechanical jam by increasing the concentration of the atoms, i.e., pressure [2]. Both frustrated magnets and the mechanical jam have a large number of metastable states, other than their ground states, in the vicinity of their liquid states. The configurational entropy of these states ranges from extensive, as in mechanical jams and coplanar states of the kagome antiferromagnet [15,63], to subextensive as in the locally collinear states of an ideal SCGO [5]. Both types of systems are expected to feature a relatively shallow energy landscape of accessible states due to their proximity to a uniform liquid state. It is interesting to note a possibly related observation that the ensemble of metastable states in self-generated Coulomb glasses is shallow compared with more ordinary electron glasses relying on quench disorder [98].

The two fundamentally different trees studied here can be cast in the framework of complex networks [99,100]. The spin glass's hierarchical energy landscape (even with a fractal structure) resembles the so-called scale-free network [100], proposed to explain internet connections and ecological and neural networks [101]. In this network, there are highly connected dominating nodes, each of which corresponds to the global minimum of a rugged funnel. On the other hand, the spin jam's nonhierarchical landscape corresponds to a network consisting of weakly connected clusters that are homogenous on a larger scale. 


\section{Chapter 7}

\section{Universal Scaling of memories}

\subsection{Motivation}

Magnetic glassy systems present a unique opportunity for searching possible universal phenomena associated with glassy behaviors. This is because glass phase exists in a wide range of magnetic materials that are described by seemingly very different spin interactions. The most well-known common features of the magnetic glassy behaviors are the lack of long-range magnetic order and the field-cooled (FC) and zero-field-cooled (ZFC) hysteresis found in the bulk susceptibility $[102,103]$. The term spin glass was coined in 1970s to describe the low-temperature behaviors of dilute magnetic alloys that are made of nonmagnetic metals with low concentrations of magnetic impurities [102,104]. The canonical glassy behaviors are manifested in intriguing phenomena called aging, rejuvenation, and memory effects [90]. While aging simply refers to the time-span dependence of relaxation phenomena in the glassy state, rejuvenation describes the rethermalization whenever the system is further cooled after waiting at some temperature. The states accessed while aging can be retrieved upon re-heating, which is called memory effect. Several theories have been proposed to understand the physics of the spin glass.

Various systems other than the dilute magnetic alloys also exhibit the aforementioned characteristic glassy behaviors at low temperatures, even when the magnetic moments are densely populated. For example, glassy behaviors have been observed in the phase diagrams of high-temperature superconducting materials, cuprates $[105,106]$ and ironbased superconductors [107]. Another example is the so-called spin-orbit Mott insulators, $\mathrm{Li}_{2} \mathrm{RhO}_{3}[108,109]$ and $\mathrm{Na}_{2} \mathrm{Ir}_{1-\mathrm{x}} \mathrm{Ti}_{\mathrm{x}} \mathrm{O}_{3}$ [110], which exhibit anisotropic Kitaev-type 
exchange interactions. Yet another is a set of geometrically frustrated magnets, pyrochlores such as $\mathrm{Y}_{2} \mathrm{Mo}_{2} \mathrm{O}_{7}$ [19], spinels such as $\mathrm{ZnFe}_{2} \mathrm{O}_{4}$ [87] , and the quasi-two-dimensional bipyramid compounds $\mathrm{SrCr}_{9 \mathrm{p}} \mathrm{Ga}_{12-9 \mathrm{p}} \mathrm{O}_{19}$ (SCGO) [4,5,32,72] and $\mathrm{BaCr}_{9 \mathrm{p}} \mathrm{Ga}_{12-9 \mathrm{p}} \mathrm{O}_{19}$ (BCGO) [47]. We emphasize that the magnetic interactions of these systems seem to be quite different in nature. For instance, the parent compound of high- $\mathrm{T}_{\mathrm{c}}$ superconductors $\mathrm{La}_{2}$ ${ }_{x} \mathrm{Sr}_{x} \mathrm{CuO}_{4}(\operatorname{LSCO}(x))$ is a Mott insulator with a conventional Neel spin order [111]. The entire magnetic excitation spectrum of $\mathrm{La}_{2} \mathrm{CuO}_{4}$ can be understood by an effective spin Hamiltonian with dominant nearest-neighbor antiferromagnetic coupling constant $J=104$ $\mathrm{meV}$ [112]. The iron chalcogenide $\mathrm{Fe}_{1+\mathrm{y}} \mathrm{Te}$ displays a bi-collinear antiferromagnetic stripe order $[113,114]$. Magnetic interactions in the two spin-orbit Mott insulators, $\mathrm{Li}_{2} \mathrm{RhO}_{3}$ and $\mathrm{Na}_{2} \mathrm{Ir}_{1-\mathrm{x}} \mathrm{Ti}_{\mathrm{x}} \mathrm{O}_{3}$ are dominated by highly anisotropic Kitaev exchange couplings [115,116]. Remarkably, despite their different nature of magnetic interactions, all the systems show the same FC-ZFC hysteresis at low temperatures. A natural question to ask is whether or not there is a unifying concept that can unite and also classify these various glassy magnets.

\subsection{Methodology}

Here, we address this issue by investigating memory effects of several of the aforementioned exemplary systems using the bulk magnetization measurements. We performed thermo-remanent magnetization (TRM) $[88,89,91]$ measurements on five different compounds, which can be divided into three categories: (1) the high-temperature superconducting materials, cuprates and Fe-chalcogenides, (2) Kitaev-model-related systems $\mathrm{Li}_{2} \mathrm{RhO}_{3}$ and $\mathrm{Na}_{2} \mathrm{Ir}_{1-x} \mathrm{Ti}_{x} \mathrm{O}_{3}$, and (3) a semi-conducting pyrochlore $\mathrm{Y}_{2} \mathrm{Mo}_{2} \mathrm{O}_{7}$. Intriguingly, despite their distinct microscopic Hamiltonians, all of them exhibit unconventional glassy behaviors in the TRM measurements, that are weak and broad shoulder-like memory effects as in the prototype spin jam compounds SCGO/BCGO, starkly contrasting the strong and dip-like memory effects observed in the canonical spin glass such as $\mathrm{CuMn} 2 \%$. Interestingly, all the data can be well reproduced by a modified stretched exponential function of $\left\{1-\exp \left(-\left(\frac{t_{w}}{\tau}\right)^{1-n}\right)\right\}$. More importantly, all the densely populated magnets except $\mathrm{Y}_{2} \mathrm{Mo}_{2} \mathrm{O}_{7}$ yield an exponent of $1-n \approx 0.6(1)$. This value is different from $1-n \approx 1 / 3$ of dilute magnetic alloys [117--119] that was ascribed 
to their hierarchical and fractal energy landscape [120--123], and is also different from 1 $n=1$ of the conventional Debye relaxation expected for a crystal. Based on these results, we argue that the glass magnets can be categorized into two distinct classes with different relaxation behaviors characterized by the exponent: $1-n \approx 1 / 3$ for glassy magnets with hierarchical energy landscape and $1-n \approx 0.6(1)$ for the ones with non-hierarchical energy landscape.

\subsection{Universal Memory Behavior}

The TRM measurement is the most effective way to probe the memory effects in detail as explained in Appendix A.8 and as shown in the comparative study of SCGO/BCGO and the canonical spin glass $C u \mathrm{Mn} 2 \%$ in Chapter 6 [124]. While a dip-like memory effect with clear rejuvenation was observed, as expected, in $C u \mathrm{Mn} 2 \%$, a shoulder-like memory effect seen in SCGO/BCGO implies lack of rejuvenation. Figure 7.1 shows the TRM data obtained from five different compounds: (a) $\mathrm{Fe}_{1.02} \mathrm{Se}_{0.15} \mathrm{Te}_{0.85}$, (b) $\mathrm{La}_{2-x} \mathrm{Sr}_{x} \mathrm{CuO}_{4}$ (LSCO( $x=0.04)$ ), (c) $\mathrm{Li}_{2} \mathrm{RhO}_{3}$, (d) $\mathrm{Na}_{2} \mathrm{Ir}_{0.89} \mathrm{Ti}_{0.11} \mathrm{O}_{3}$, and (e) $\mathrm{Y}_{2} \mathrm{Mo}_{2} \mathrm{O}_{7}$. These TRM data were taken after waiting at the waiting temperature $T_{w} \sim 0.7 T_{f}$ for several different waiting times ranging from 1.5(5) min to maximally 100 hours. For all systems aging and memory effect appears, i.e., the magnetization decreases near $T_{w}$ when the measurements were performed after waiting. The memory effect gets enhanced as the waiting time, $t_{w}$, increases. Surprisingly, $\mathrm{Fe}_{1.02} \mathrm{Se}_{0.15} \mathrm{Te}_{0.85}$ and $\mathrm{LSCO}(x=0.04)$ whose parent compounds, FeTe and $\mathrm{La}_{2} \mathrm{CuO}_{4}$, respectively, are long-range ordered state, i.e., spin solid, exhibit very weak memory effects. The memory effects in both systems are negligible for short waiting time $t_{w} \leq 6 \mathrm{~min}$. For $t_{w} \gtrsim 1 \mathrm{hr}$, both systems show a very weak and broad shoulder appearing around $T_{w}$ (see Figure 7.1 (a) and (b)), regardless of how large $t_{w}$ is. For $\mathrm{Fe}_{1.02} \mathrm{Se}_{0.15} \mathrm{Te}_{0.85}$, the memory effect even seems to saturate for $t_{w} \gtrsim 30 \mathrm{hrs}$ (Figure 7.1 (a)). Similar weak shoulder-like memory effects were also observed in the spin-orbit Mott insulators, $\mathrm{Li}_{2} \mathrm{RhO}_{3}$ and $\mathrm{Na}_{2} \mathrm{Ir}_{0.89} \mathrm{Ti}_{0.11} \mathrm{O}_{3}$ (see Figure 7.1 (c) and (d), respectively). Note that, similarly to the two superconductivity-related systems, the two Kitaev-model-related systems also exhibit negligible memory effects for short waiting time $t_{w} \leq 6 \mathrm{~min}$.

The weak shoulder-like memory effects have been observed in frustrated magnets, SCGO and BCGO (see Chapter 6 ), that are in the vicinity of spin liquid, and here we show that 
another frustrated magnet, $\mathrm{Y}_{2} \mathrm{Mo}_{2} \mathrm{O}_{7}$, also exhibits similar features (Figure 7.1(e)). These data clearly show that the weak shoulder-like memory effect is universal in these densely populated magnets, regardless of their magnetic interactions. It is in stark contrast to the canonical spin glass such as $\mathrm{CuMn} 2 \%$, where the memory effects in the magnetization curve were readily seen even for such short waiting times as $t_{w}=1.5(5) \mathrm{min}$ (see Figure 7.3 (a)), and the effects become sharp and strong, appearing as a large dip at $T_{w}$ for $t_{w} \geq 3$ hrs [124].

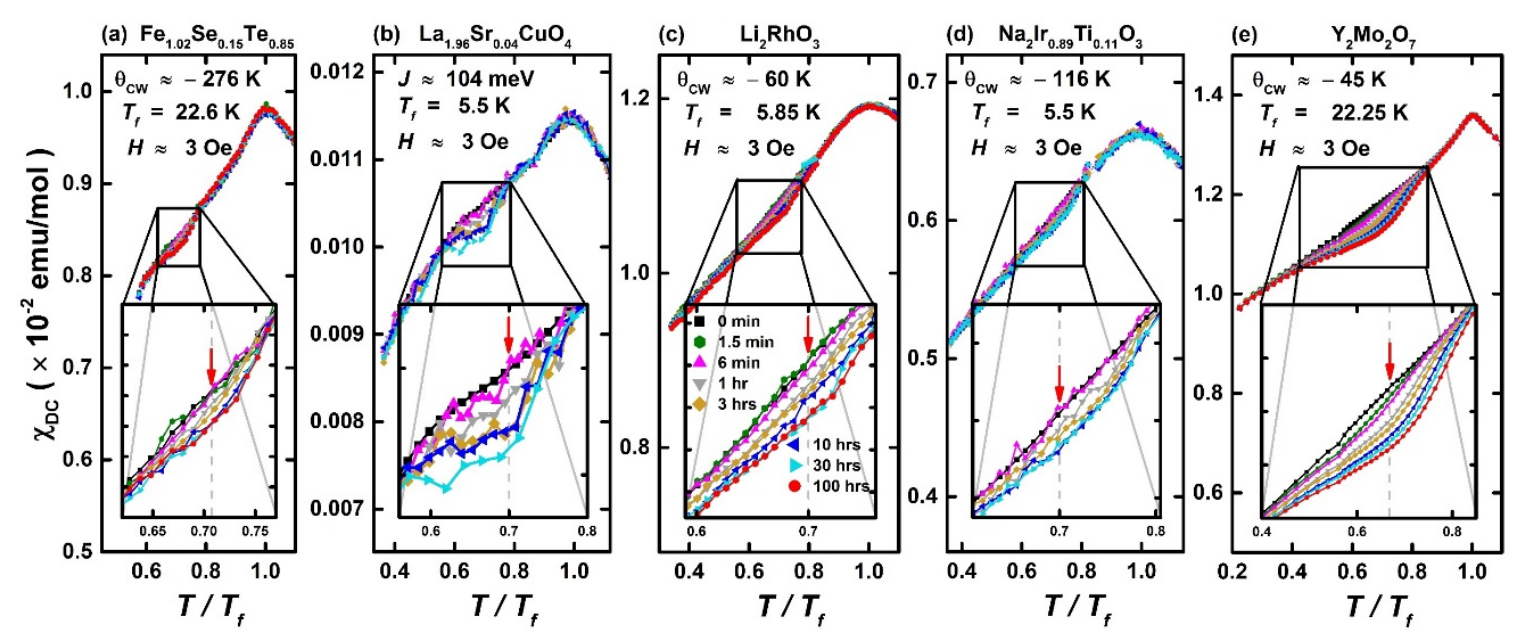

Figure 7.1 Memory Effect as a function of waiting time.

Bulk susceptibility, $\chi_{D C}=M / H$, where $M$ and $H$ are magnetization and applied magnetic field strength, respectively, obtained from (a) $\mathrm{Fe}_{1.02} \mathrm{Se}_{0.15} \mathrm{Te}_{0.85}$ (b) $\mathrm{La}_{1.96} \mathrm{Sr}_{0.04} \mathrm{CuO}_{4}$, (c) $\mathrm{Li}_{2} \mathrm{RhO}_{3}$ (d) $\mathrm{Na}_{2} \mathrm{I}_{0.89} \mathrm{Ti}_{0.11} \mathrm{O}_{3}$ and (e) $\mathrm{Y}_{2} \mathrm{Mo}_{2} \mathrm{O}_{7}$, with $H=30 e$. Symbols and lines with different colors indicate the data taken with different waiting times, $t_{w}$, ranging from zero to $100 \mathrm{hrs}$, at $T_{w} / T_{f} \sim 0.7$ where $T_{w}$ and $T_{f}$ are the waiting and the freezing temperature, respectively. For $\mathrm{Fe}_{1.02} \mathrm{Se}_{0.15} \mathrm{Te}_{0.85}$, the Curie-Weiss Temperature $\theta_{c w}$ was estimated by fitting its high-T susceptibility data as shown in Fig. S1A in the Supplementary Information. For $\mathrm{La}_{1.96} \mathrm{Sr}_{0.04} \mathrm{CuO}_{4}$, the high-T susceptibility does not follow the simple Curie-Weiss law (see Fig. S1b in Supplementary Information). In order to show how strong the magnetic interactions are in LSCO, we quote the coupling constants of the parent compound $\mathrm{La}_{2} \mathrm{CuO}_{4}$ that were experimentally determined by inelastic neutron scattering (ref. [112] ); the antiferromagnetic nearest-neighbor $J \approx$ $104 \mathrm{meV}$ and the ferromagnetic next-nearest-neighbor $J^{\prime} \approx-18 \mathrm{meV} . \theta_{c w}$ for $\mathrm{Li}_{2} \mathrm{RhO}_{3}$, $\mathrm{Na}_{2} \mathrm{Ir}_{0.89} \mathrm{Ti}_{0.11} \mathrm{O}_{3}$ and $\mathrm{Y}_{2} \mathrm{Mo}_{2} \mathrm{O}_{7}$ were taken from ref. 9 [109], [116] and [64], respectively.

Figure 7.2 (a) summarizes the $t_{w}$ dependence of the memory effect for the densely populated magnets along with the canonical spin glass $\mathrm{CuMn} 2 \%$. The relative change of the magnetization $\Delta M_{r e l}=\left(M_{\text {ref }}-M\right) / M_{\text {ref }}$ induced by the aging, in which $M$ and $M_{\text {ref }}$ are the magnetizations with and without waiting, respectively, is plotted. Overall, it is clear that the memory effect is much weaker in densely populated magnets than in the canonical 
spin glass. Firstly, $\Delta M_{\text {rel }}$ of $t_{w} \geq 30 \mathrm{hrs}$ for all the densely populated magnets except $\mathrm{Y}_{2} \mathrm{Mo}_{2} \mathrm{O}_{7}$ is smaller than $\Delta M_{\text {rel }}$ of $t_{w}=6$ min for $C u \mathrm{Mn} 2 \%$. Secondly, for $t_{w} \leq 6 \mathrm{~min}$, most of them show negligible memory effects as shown in Figure 7.2 (a) and Figure 7.1. Thirdly, the memory effect of the spin jams except $\mathrm{Y}_{2} \mathrm{Mo}_{2} \mathrm{O}_{7}$ seems to saturate for $t_{w} \geq$ $30 \mathrm{hrs}$, while for $C u \mathrm{Mn} 2 \%$ it seems to keep increasing with increasing $t_{w}$ over the time period.
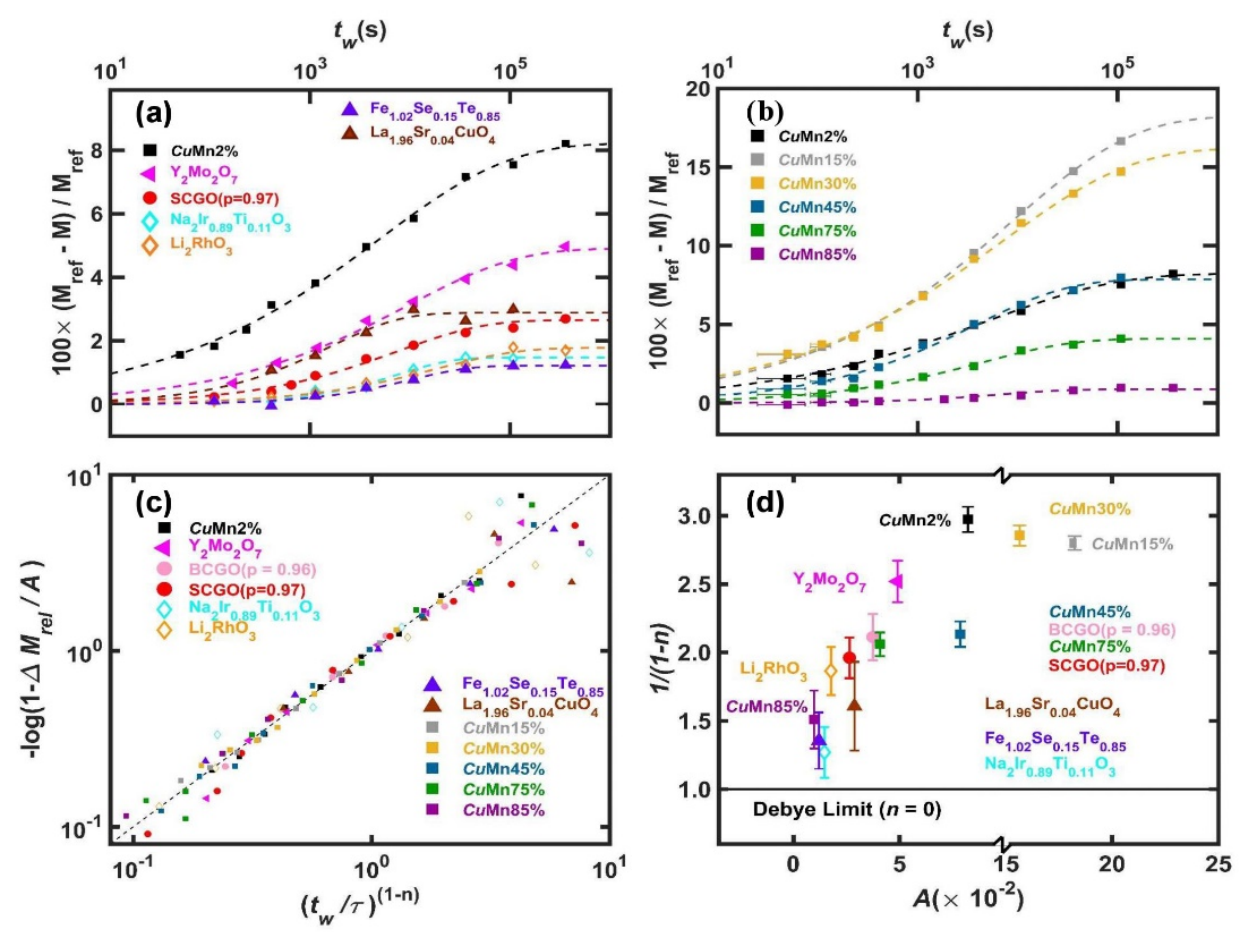

Figure 7.2 Summarizing the memory effect.

From the data shown in (a) Figure 7.1 and (b) Figure 7.3, the aging effect was quantified for the eleven systems by plotting the relative change of the magnetization $\Delta M_{r e l}=\left(M_{r e f}-M\right) / M_{r e f}$ where $M_{\text {ref }}$ is the magnetization without waiting, and it was plotted as a function of $t_{w}$ in a log scale. The aging effects of a spin jam prototype, $\mathrm{SrCr}_{9 \mathrm{p}} \mathrm{Ga}_{12-9 \mathrm{p}} \mathrm{O}_{19}(\mathrm{SCGO}(\mathrm{p}=0.97))$, and a spin glass prototype $C u \mathrm{Mn} 2 \%$ were taken from Ref. [124], except the $t_{w}=1.5(5) \mathrm{min}$ data are new and are also plotted here for comparison. Each set of $\Delta M_{r e l}\left(t_{w}\right)$ for each sample shown in panels (a) and (b) was fitted to the modified stretched exponential function, Equation 7.1. After the fitting, in (c) $-\log \left(1-\Delta M_{\text {rel }} / A\right)$ was plotted as a function of $\left(t_{w} / \tau\right)^{1-n}$ in a log-log scale. (d) The degree of aging, $A$, and the inverse exponent, $1 /(1-n)$, obtained for all the samples are plotted against each other.

It is interesting that the densely populated $\mathrm{Y}_{2} \mathrm{Mo}_{2} \mathrm{O}_{7}$ exhibits both spin glass and spin jam behaviors. This is probably due to the fact that $\mathrm{Y}_{2} \mathrm{Mo}_{2} \mathrm{O}_{7}$ is a semi-conductor evidenced by its resistivity of $\rho \sim 10^{-2} \Omega \cdot \mathrm{cm}$ at $300 \mathrm{~K}$, and has an unquenched orbital degree of freedom 
[64,125]. As a result, $\mathrm{Y}_{2} \mathrm{Mo}_{2} \mathrm{O}_{7}$ is not a typical frustrated antiferromagnet, which is manifested in the relatively small frustration index $f=\Theta_{C W} / T_{f} \cong 2.3$ that is two orders of magnitude smaller than that of SCGO.

In search of possible underlying scaling behavior, we have fitted the $t_{w}$ dependence of $\Delta M_{\text {rel }}$ to the following phenomenological function

$$
\Delta M_{r e l}\left(t_{w}\right)=A\left\{1-\exp \left(-\left(\frac{t_{w}}{\tau}\right)^{1-n}\right)\right\}
$$

which is modified from the stretched exponential function that was proposed to describe relaxation phenomena in glassy systems [117--123]. The modification made here is to take into account the experimental observation that $\Delta M_{\text {rel }}$ seems to saturate for long waiting times. Here $A=\Delta M_{r e l}\left(t_{w} \rightarrow \infty\right)$ is a measure of degree of aging, $\tau$ is a microscopic time scale for relaxation dynamics. A positive non-zero exponent $n$ would tell us how much the relaxation deviates from the conventional Debye behavior $(n=0)$. The exponent $1-n$ can be related to critical exponents for the spin glass transition within the framework of a random cluster model [121,122]. For example, assuming that the growth of clusters involves no conserved mode, the droplet model predicts an exponent $1-n=1 / 2$ [126] . The dashed lines in Figure 7.2 (a) are the fits of the experimental data to Equation 7.1 for all the materials. It is remarkable that the same phenomenological function, albeit with different parameters, reproduces all the data of both spin jams and spin glass over the wide range of the waiting time. This indicates that a universal scaling may be in play in the aging or relaxation phenomena of all glassy magnets, as shown in Figure 7.2 (c).

The difference between the spin glass and spin jam is clearly manifested in different parameters in Figure 7.2 (d). For spin glass $C u M n 2 \%$, the exponent $1-n \approx 1 / 3$ that deviates significantly from the conventional Debye behavior of $1-n=1$. This is consistent with the previous studies on several other dilute magnetic alloys such as $C u \mathrm{Mn} 1 \%$ and $A g \mathrm{Mn} 2.6 \%$ [117], NiMn23.5\% [118], Au90 $\mathrm{Fe}_{10}$ [119]. This deviation observed in the spin glasses was ascribed to the underlying hierarchically constrained dynamics [120--123]. On the other hand, the densely populated glassy magnets, SCGO, $\mathrm{Fe}_{1.02} \mathrm{Se}_{0.15} \mathrm{Te}_{0.85}$, LSCO, and the two spin-orbit Mott insulators, yield the exponent of 1 - 
$n \approx 0.6(1)$, indicating a smaller deviation from the conventional Debye relaxation. This implies that their energy landscapes are not hierarchical as in the canonical spin glass. These are summarized in Figure 7.2 (d) in which the exponent $1-n$ is plotted as a function of the degree of aging, $A$. We note that there is a positive correlation between the deviation from the Debye limit and the degree of aging.

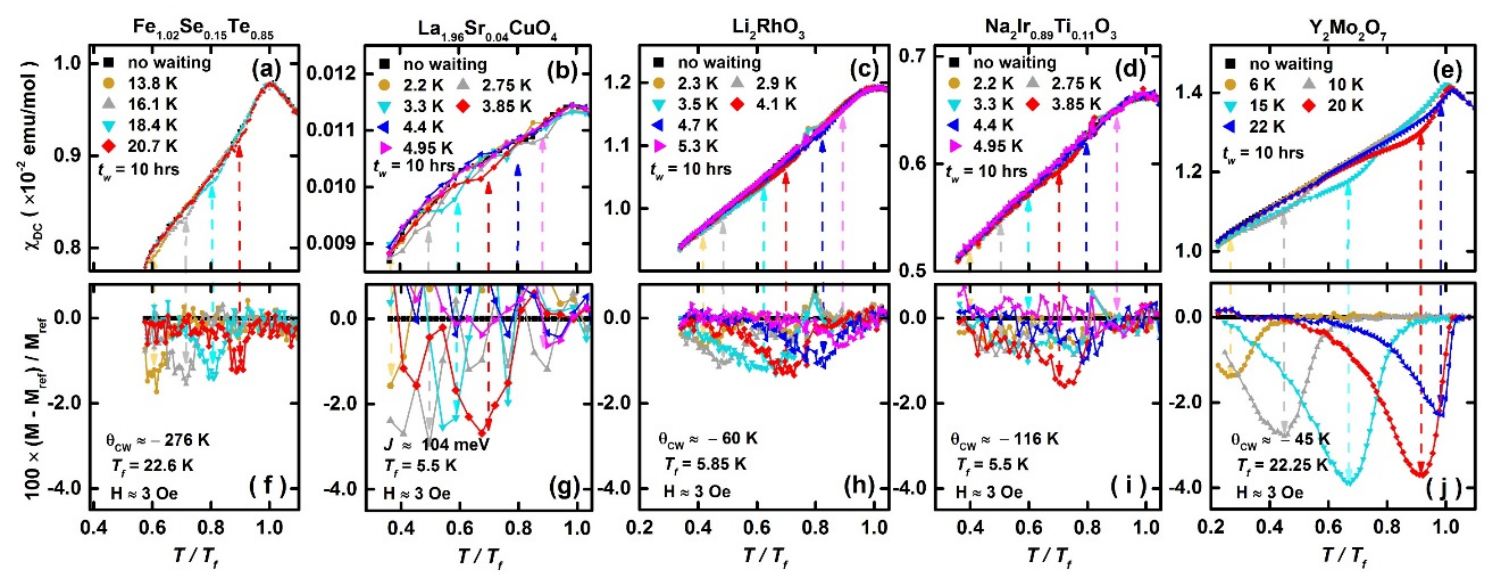

Figure 7.3 Temperature Dependence of memory effect.

$\chi_{D C}$ and $\left(M_{\text {ref }}-M\right) / M_{\text {ref }}$ measured for (a) $\mathrm{Fe}_{1.02} \mathrm{Se}_{0.15} \mathrm{Te}_{0.85}$ (b) $\mathrm{La}_{1.96} \mathrm{Sr}_{0.04} \mathrm{CuO}_{4}$, (c) $\mathrm{Li}_{2} \mathrm{RhO}_{3}$ (d) $\mathrm{Na}_{2} \mathrm{Ir}_{0.89} \mathrm{Ti}_{0.11} \mathrm{O}_{3}$ and (e) $\mathrm{Y}_{2} \mathrm{Mo}_{2} \mathrm{O}_{7}$, with $t_{w}=10 \mathrm{hrs}$, at various waiting temperatures.

Moreover, Figure 7.3 shows that for the spin jam systems the memory effect with $t_{w}=$ $10 \mathrm{hrs}$ is maximal when the waiting temperature $T_{w} \sim 0.7 T_{f}$ and it becomes weaker for other values of $T_{w}$ over a wide range of $T_{w}$.

\section{4 $C u_{1-x} \mathrm{Mn}_{x}$ system as a function of $x$}

To further support the aforementioned scenario, we have performed the TRM measurements on $C u_{1-x} \mathrm{Mn}_{x}$ as a function of the Mn concentration, $x$. This series of compounds provides an excellent platform also to investigate how the spin glass is connected with the spin jam, and eventually magnetic ordered states. On one hand, $\mathrm{Cu}$ ${ }_{x} \mathrm{Mn}_{x}$ is a canonical spin glass for small $x$. On the other hand, pure Mn exhibits a long-range spin-density-wave (SDW) order at low temperatures. The magnetic ground state of samples with large $x$ thus can be viewed as large domains of SDW order disrupted by non-magnetic $\mathrm{Cu}$ atoms, similar to that observed in the densely populated magnets such as $\mathrm{Fe}_{1.02} \mathrm{Se}_{0.15} \mathrm{Te}_{0.85}$ and $\mathrm{La}_{1.96} \mathrm{Sr}_{0.04} \mathrm{CuO}_{4}$. 

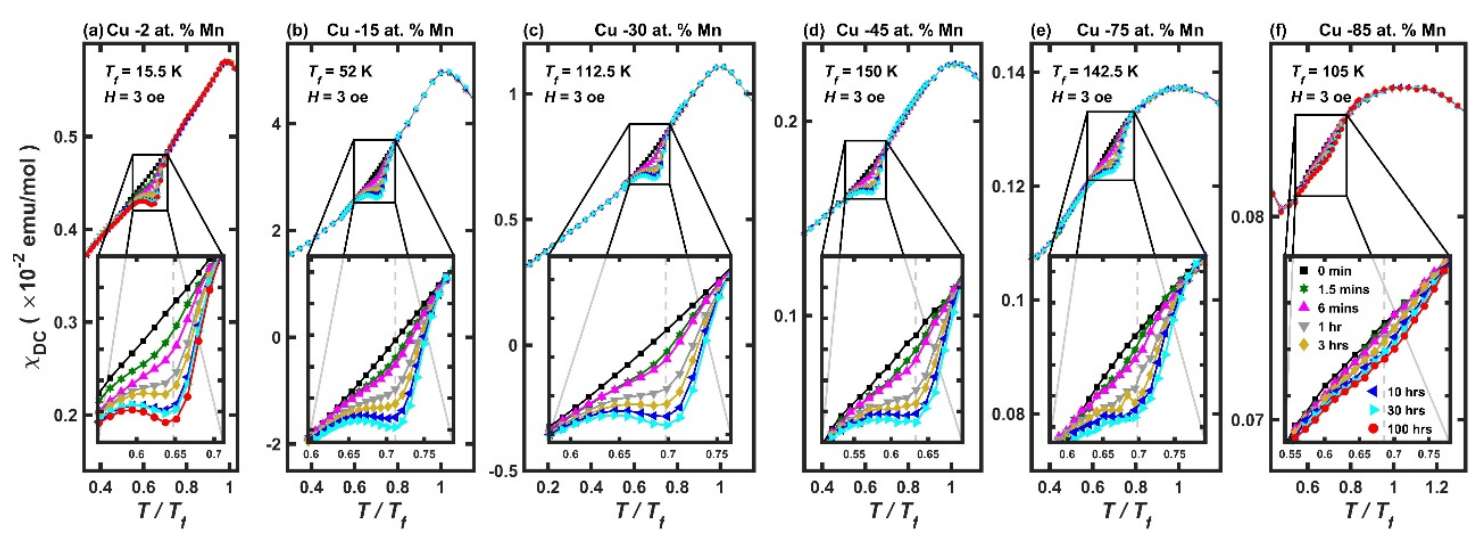

Figure 7.4 Memory Effect of $\mathrm{Cu}-\boldsymbol{x}$ at. \% Mn samples as a function of $\boldsymbol{t}_{\boldsymbol{w}}$.

Bulk susceptibility, $\chi_{D C}=M / H$, where $M$ and $H$ are magnetization and applied magnetic field strength, respectively, obtained from $C u_{1-x} \mathrm{Mn}_{x}$ with (a) $x=0.02$, (b) $x=0.15$, (c) $x=0.30$, (d) $x=0.45$, (e) $x=0.75$ and (f) $x=0.85$, with $\mathrm{H}=3$ Oe. Symbols and lines with different colors indicate the data taken with different waiting times, $t_{w}$, ranging from zero to $100 \mathrm{hrs}$, at $T_{w} / T_{f} \sim 0.7$ where $T_{w}$ and $T_{f}$ are the waiting and the freezing temperature, respectively.

As shown in Figure 7.3 (a), (b), (c) and (d), for dilute alloys with small values of $x \lesssim 0.45$, the data exhibits prominent dip behaviors, i.e., the presence of rejuvenation. As $x$ increases further, the dip behavior is gradually replaced with the shoulder behaviors, i.e., lack of rejuvenation, similar to spin jam (see Figure 7.3 (e) and (f)). The crossover seems to occur at $x \sim 0.45$ that is close to the percolation threshold for a three-dimensional system [63]. Note the non-monotonic behavior of the degree of aging $A=\Delta M_{r e l}\left(t_{w} \rightarrow \infty\right)$ that maximizes at $x \sim 0.15$. The initial growth of $\mathrm{A}$ for small $x$ is related to the increasing number of magnetic impurities, giving rise to a stronger magnetic signal. For very large $x$ where the system is in the spin jam regime, the degree of aging is expected to decrease as observed for $x=0.75$ and 0.85 shown in Figure 7.3 (e) and (f) respectively. Thus, even though the exact value of $x$ for the maximal $\mathrm{A}$ is determined by the balancing between the exact nature of the magnetic interactions and the magnetic concentration, the maximum of A should occur most likely somewhere close to the middle of $x=0$ and the percolation threshold, which is qualitatively consistent with the observed value of $x \sim 0.15$.

Surprisingly, regardless of $x, \Delta M_{\text {rel }}$ of $C u_{1-x} \mathrm{Mn}_{x}$ follows the same stretched exponential relaxation function, as shown in Figure 7.2 (b), but with varying values of the exponent, $1-n$, from $0.34(1)$ for $x=0.02$ to $0.66(9)$ for $x=0.85$ (see Figure $7.2(\mathrm{~d})$ ). And thus, all their $\Delta M_{\text {rel }}$ can be collapsed into a same function, once the waiting time is properly scaled, and 
it is so even with those of the densely populated glassy systems, as shown in Figure 7.2 (c). The change in the exponent, $1-n$, as a function of $x$ clearly shows that the glassy state of the dilute magnetic alloy (for small $x$ ) is replaced by a glassy state for large $x$ similar to the one observed in the densely populated magnets (see Figure 7.2 (d)) Interesting, the crossover occurs as the magnetic concentration go beyond the percolation threshold [63]. This clear crossover phenomenon strongly indicates that there are two distinct glassy states: spin glass and spin jam.

\subsection{Clues from Neutron Scattering}

Why do the densely populated systems exhibit the large exponent $1-n \approx 0.6(1)$ similar to the quantum-fluctuation-induced spin jam SCGO, compared to the canonical spin glass state of dilute magnetic alloys? A clue comes from neutron scattering studies; the magnetic structure factor, $I(Q)$, of all the densely populated magnets studied here exhibit prominent peaks that are centered at a non-zero momentum $(Q)$ corresponding to short-range spin correlations, as those of the frustrated magnets SCGO [34,72] and BCGO [47]. This indicates that those systems have dominant antiferromagnetic interactions between localized spins and short-range spin correlations. For example, the cuprate $[127,128]$ and iron chalcogenide $[129,130]$ exhibit strong incommensurate peaks near the antiferromagnetic ordering wave vector of their parent compounds. As shown in Figure 7.4 (b), the spin-orbit Mott insulator $\mathrm{Na}_{2} \mathrm{Ir}_{0.89} \mathrm{Ti}_{0.11} \mathrm{O}_{3}$ exhibit a prominent peak centered at $Q=$ $0.87(2) \AA^{-1}$. The common characteristics of the antiferromagnetic and short-range magnetic structure factor starkly contrast with the nearly featureless magnetic structure factor of the spin glass $\mathrm{CuMn} 2 \%$, as shown in Figure 7.4 (d). In the dilute magnetic alloys such as $\mathrm{CuMn} 2 \%$, magnetic impurities interact among themselves through the RudermanKittel-Kasuya-Yosida (RKKY) interactions that are mediated by the itinerant electrons. The RKKY interactions are long-ranged and oscillate from ferromagnetic to antiferromagnetic as a function of the distance. As a result, the random distances among the magnetic moments lead to their random interactions that even change the sign, resulting in the featureless magnetic structure factor. 

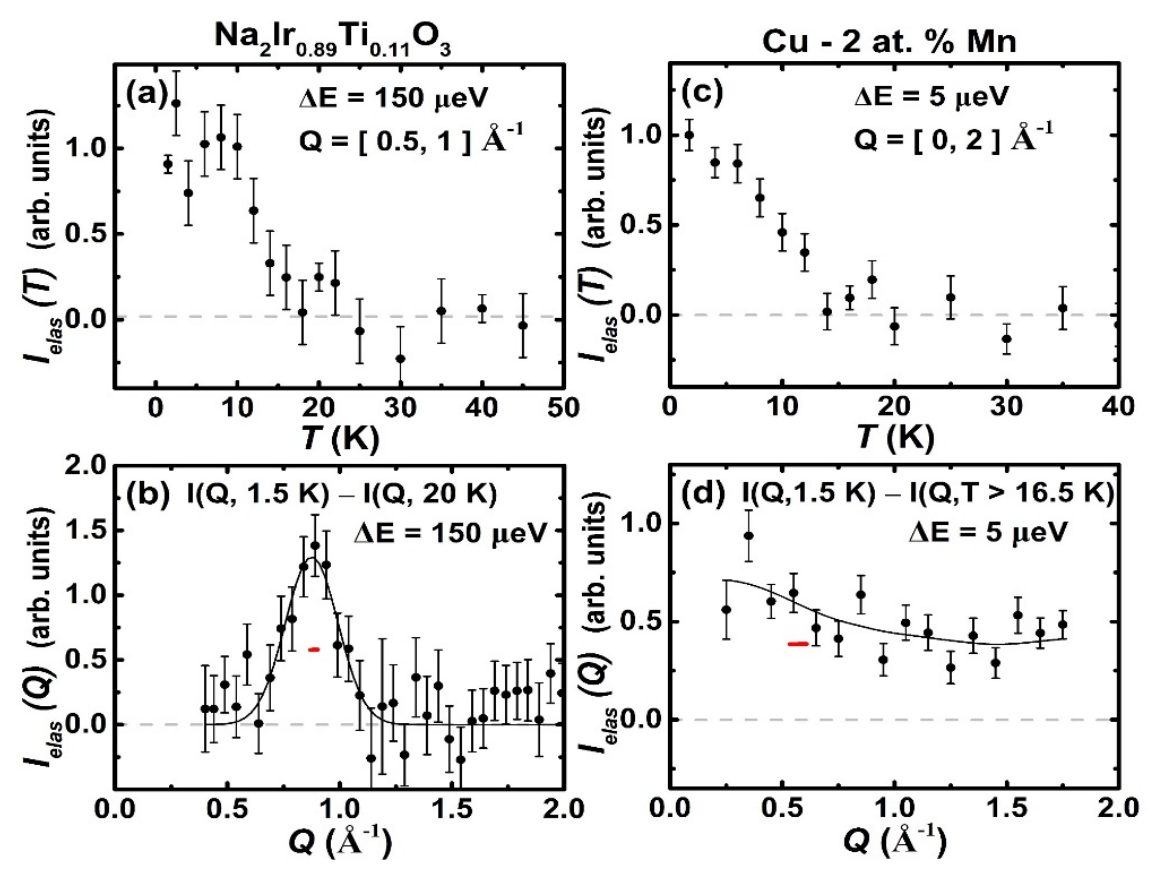

Figure 7.5 Neutron scattering measurements of NITO and $\mathrm{CuMn2 \%}$.

(a) T-dependence, $I_{\text {elas }}(T)$, and (b) $Q$-dependence, $I_{\text {elas }}(Q)$, of elastic magnetic neutron scattering intensity obtained from $\mathrm{Na}_{2} \mathrm{Ir}_{0.89} \mathrm{Ti}_{0.11} \mathrm{O}_{3}$. The measurements were done at the Cold Neutron Chopper Spectrometer (CNCS) at the Spallation Neutron Source (SNS). (c) T-dependence, $I_{\text {elas }}(T)$, and (d) $Q$-dependence, $I_{\text {elas }}(Q)$, of elastic magnetic neutron scattering intensity obtained from the magnetic alloy $C u \mathrm{Mn} 2 \%$. The measurements were done at the Backscattering Spectrometer (BASIS) at SNS. For both $I_{\text {elas }}(Q)$ in (b) and (d), the non-magnetic background was determined from the data above the freezing temperature and subtracted from the base temperature data. The black solid line in (b) is the fit of the magnetic peak centered at $Q=0.87 \AA^{-1}$ to a simple Gaussian, while the line in (d) is a guide to eyes. The red horizontal bar at the center of the peak in (b) represents the instrument Q-resolution, $d Q \approx 0.06 \AA^{-1}$, that was determined by fitting a nearby Bragg peak centered at $1.2 \AA^{-1}$.

The featureless $I(Q)$ of $C u \mathrm{Mn} 2 \%$ is consistent with the real-space droplet model for spin glass $[126,131]$ in which low-energy excitations are dominated by connected spin clusters of arbitrary length scales. The real-space clusters or droplets correspond to the meta-stable ground states or local minima in the energy landscape. Their arbitrary length scales and random RKKY interactions yield a multitude of energy scales, resulting in the complex hierarchical fractal energy landscape [124,132--134]. As a consequence, the spin glass exhibits the observed strong dip-like memory effect. In contrast to the droplet model for spin glass, the clusters in spin jams are more uniform in size, as evidenced by the prominent peak of $I(Q)$. This feature, combined with the short-range exchange spin Hamiltonian, leads to a narrowly distributed energy scale, and the weak memory effect as observed in our susceptibility measurements. 
The distinct nature of the two magnetic glass phases, spin glass and spin jam, also manifests in their characteristically different low energy excitations. The thermodynamic behavior of canonical spin glass at low temperatures is dominated by thermally active clusters or droplets, particularly those with a free energy less than or of the same order of $k_{B} T$ where $k_{B}$ is the Boltzmann constant. The fact that there is a finite density of clusters with limiting zero free energy naturally leads to the linear- $T$ specific heat $[17,126]$, which is a signature of canonical spin glass. On the other hand, the low-energy excitations in spin jam are the Halperin-Saslow (HS) spin waves with finite spin stiffness over large length scales (often larger than the typical cluster sizes) $[15,16,60]$. These gapless HS modes exhibit a linear dispersion relation and are the source of a $T^{2}$ dependence of the specific heat for a twodimensional system. Indeed, such $T^{2}$ behavior has been observed in the glass phase of SCGO [32], $\mathrm{Li}_{2} \mathrm{RhO}_{3}[108,109]$, and doped $\mathrm{Na}_{2} \mathrm{IrO}_{3}$ [110].

\subsection{Summary and Conclusions}

The memory effect measurements provide crucial information about the nature of relaxation dynamics in different magnetic states, which allows us to classify the semiclassical magnetic glassy materials as shown in Figure 7.5. At the lower left corner of the triangle lies the spin solid that is realized in densely populated semi-classical magnetic materials with small disorder and weak frustration that order long-range at low temperatures with Debye relaxation. The typical energy landscape associated with a spin solid is a smooth vase with a global minimum. At the lower right corner of the triangle lies the spin glass that is realized in dilute magnetic alloys with random magnetic interactions. Its typical energy landscape is dominated by hierarchical meta-stable states that correspond to spin clusters of arbitrary length scales in real space, exhibiting hierarchical rugged funnels and fractal geometry, and the observed strong deviation from the conventional Debye relaxation. Finally, at the top corner is the new magnetic state dubbed spin jam that encompasses many densely populated compounds with short-range exchange magnetic interactions, disorder and frustration. Disorder can be either extrinsic as in LSCO, FeTeSe and $\mathrm{Na}_{2} \mathrm{Ir}_{1-x} \mathrm{Ti}_{x} \mathrm{O}_{3}$, or intrinsic due to quantum fluctuations as in SCGO and BCGO [4,5]. One salient feature of the spin jam, represented by a nonhierarchical energy landscape with a wide and nearly flat but rough bottom, is the lack of widely distributed energy and time 
scales. This in turn leads to a significantly weaker memory effect and the relaxation exponent that is closer to the Debye exponent than that of the spin glass, as observed in our experiments. Remarkably, the canonical spin glass $C u_{1-x} \mathrm{Mn}_{x}$ with small $x$ crosses over to the spin jam state when the magnetic concentration $x$ increases beyond the percolation threshold.

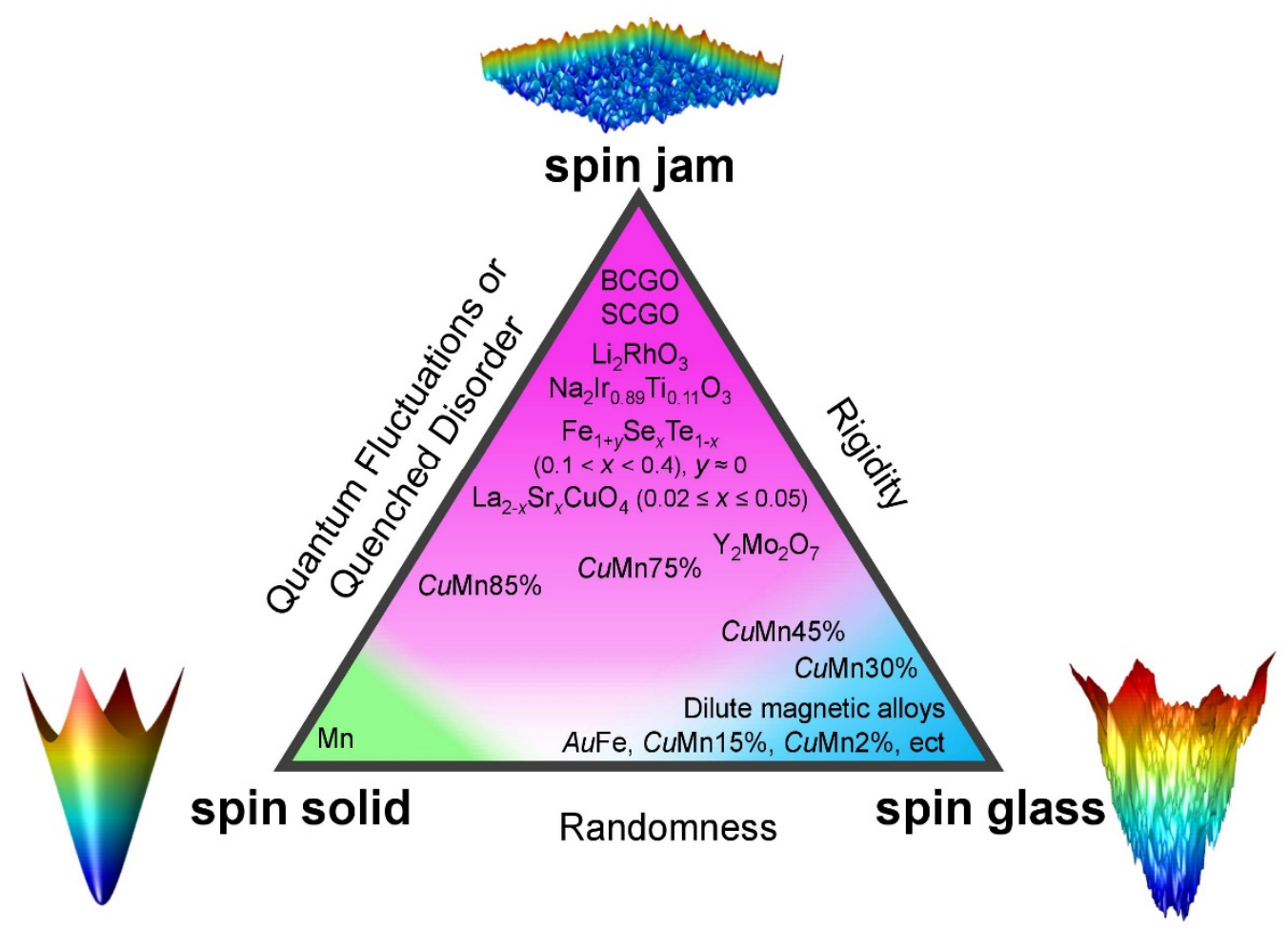

Figure 7.6 Schematic phase diagram.

Classification of semi-classical magnetic states into three distinct phases, spin solid, spin jam, and spin glass, was made based on the memory effect.

Our classification of a wide range of semi-classical glassy magnets based on nonequilibrium relaxation dynamics to two distinct states has implication to other nonmagnetic structural glasses. Indeed, recent studies have found two distinct low-frequency modes in structural glass: one related to a hierarchical energy landscape and the other related to jamming [135--137]. The rather distinct aging and memory behaviors observed in the spin glass and jam might also shed light on the relationship between nonequilibrium dynamics and connectivity among elementary interacting agents in networks and socioeconomic systems [101]. 


\section{Chapter 8}

\section{Comprehensive study of the dynamics of a classical Kitaev spin liquid}

\subsection{Introduction}

Quantum spin liquids (QSLs) have attracted great interest in both theoretical and experimental condensed matter physics due to their remarkable topological properties. Among many different proposals, the Kitaev model [138] defined on the honeycomb lattice, is a proto-typical two-dimensional (2D) QSL, which can be experimentally studied in iridium or ruthenium based materials [139]. However, the lack of a symmetry-breaking order parameter poses a challenge for the experimental characterization of QSLs. In the absence of a smoking-gun experiment, it is important to characterize the dynamical response of QSLs in order to identify signatures, which can guide the experimental search of these exotic states of matter [140]. The computation of dynamical correlators of interacting quantum spin systems in dimension higher than one is very challenging for state of the art techniques. For instance, the study of dynamics in the Kitaev-Heisenberg model, which is not integrable due to the additional Heisenberg interaction, was recently initiated by using a matrix-product state based $T=0$ method [141] and exact diagonalization [142]. These $T=0$ techniques can only be applied to relatively small clusters or quasi-onedimensional lattice geometry. Fortunately, the integrability of the pure Kitaev model allows for an exact calculation of the magnetic structure factor, $S(Q, \omega)$, at $T=0[143,144]$ and for a controlled numerical calculation at any finite temperature $T$ [145--147]. This remarkable property is being used to identify proximates to Kitaev liquids [140,148,149]. 
However, the actual model Hamiltonians of these materials are not integrable, so it is more challenging to assess the effect of the additional Hamiltonian terms on $S(Q, \omega, T)$.

Given the above considerations, it is relevant to ask if a semi-classical treatment can shed light on the dynamics of the Kitaev QSLs. Semi-classical treatments are very useful for describing the low-temperature properties of unfrustrated magnets, whose low energy modes are quantized spin-waves or magnons. For instance, semiclassical dynamics simulations using an appropriate quantum-classical correspondence were found to produce a good description of the intermediate and high-temperature regimes of the $2 \mathrm{D} S=5 / 2$ antiferromagnet $\mathrm{Rb}_{2} \mathrm{MnF}_{4}$, over all wavevector and energy scales, with a crossover temperature $\sim \theta_{C W} / S\left(\theta_{C W}\right.$ is the Curie-Weiss temperature) [150] . It is clear, however, that the semi-classical treatment cannot capture the intrinsically quantum mechanical nature of the low-energy excitations of quantum liquids. At first sight, this observation seems to render semi-classical approaches completely inadequate. Nevertheless, we will demonstrate that a semi-classical treatment of the Kitaev model can capture several properties of the dynamical structure factor of the $S=1 / 2$ model, including a quite remarkable agreement above the quantum to classical crossover temperature $T_{Q C}[151,152]$

The spin-S Kitaev model with $S>1 / 2$ was introduced by Baskaran et al. [153,154] and it was subsequently studied by different groups [155--158]. This model is not exactly solvable, but it preserves the $Z_{2}$ gauge structure of the $S=1 / 2$ model. The set of commuting operators

$$
W_{p}=-\sigma_{1}^{y} \sigma_{2}^{z} \sigma_{3}^{x} \sigma_{4}^{y} \sigma_{5}^{z} \sigma_{6}^{x}
$$

defined on each hexagonal plaquette of the honeycomb lattice (see Figure 8.1), is generalized to

$$
W_{p}=e^{i \pi\left(S_{1}^{y}+S_{2}^{z}+S_{3}^{x}+S_{4}^{y}+S_{5}^{z}+S_{6}^{x}\right)}
$$

for arbitrary spin $S$. An immediate consequence of this local $Z_{2}$ symmetry is that the twospin correlator $\left\langle S_{i}^{\nu} S_{j}^{\nu}\right\rangle$ is nonzero only for $i=j$ and for the nearest-neighbor (NN) sites 
connected by a $v v$ bond $(v=x, y, z) \quad[153,154]$. Consequently, both the quantum and the classical pure Kitaev models share the property of having a very short correlation length $\xi \leq a$ ( $a$ is the lattice space) for arbitrary temperature $T$.

(a)

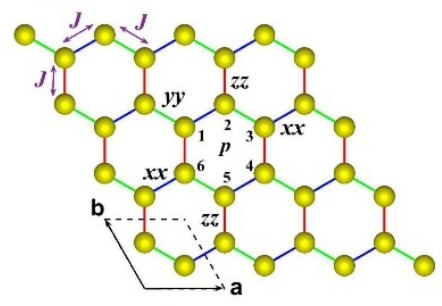

(c) (b)
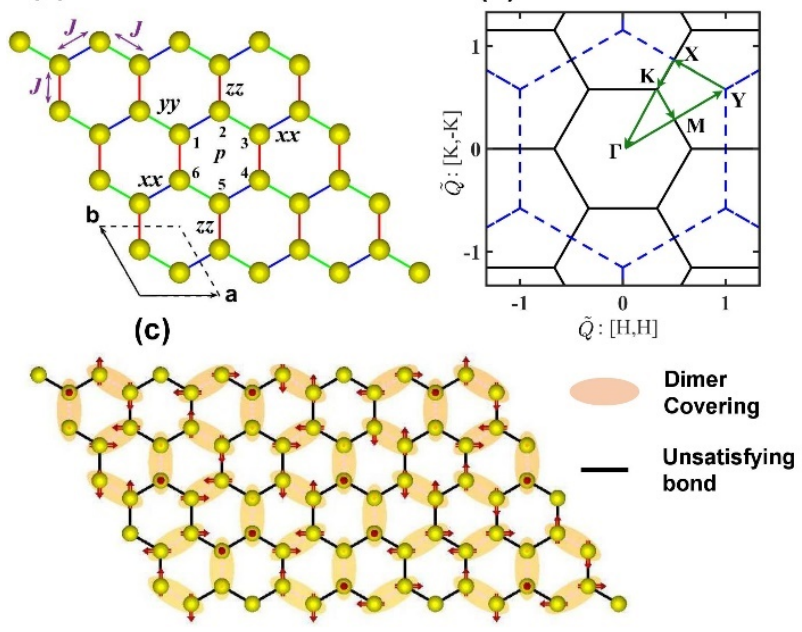

Figure 8.1 The Kitaev-Heisenberg model.

(a) Schematic illustration of the Kitaev-Heisenberg model consisting of the Heisenberg interaction $J$ and the compass-like nearest-neighbor Ising interactions, with the associated spin component for each bond depending on the bond orientations $(x x, y y$, or $z z)$. The site indices around the plaquette $p$ correspond to the denition of the $Z_{2}$ flux operator $W_{p}$ in Equation 8.2. (b) The first BZ (solid line) and the second BZ (dashed line) of the honeycomb lattice. Here, $\widetilde{\mathrm{Q}}=$ $\mathrm{Q} a / 2 \pi$. The green arrows indicate a path connecting high-symmetry points in the reciprocal space (i.e., K-Г-M-Y-X-K-M), along which we evaluate $S(\mathrm{Q}, \omega)$. (c) Example of a CN-ground state formed by antiferromagnetic dimer coverings.

As expected, the main differences between the quantum and the classical limits of the model appear in the low energy sector. While the $S=1 / 2$ version of the model has a unique QSL ground state, the ground state is massively degenerate in the classical limit. The structure of the classical ground-state manifold corresponds to an exponentially large number of isolated points in the phase space, known as the Cartesian $(\mathrm{CN})$-ground states [154], as well as continuous families of intermediate states connecting one $\mathrm{CN}$-ground state to another $[154,159]$. The $\mathrm{CN}$-ground states have each spin pointing along one of the three axes $(x, y$ or $z)$, in such a way that one of the three bonds that arrive to a common site has the minimum possible energy, while the other two have zero energy [see Figure 8.2(c)]. The zero modes associated with the continuous ground-sate degeneracy lead to a singular $\omega=0$ contribution to $S(Q, \omega)$, which is naturally absent in the $S=1 / 2$ version of the model. Instead, the $S=1 / 2$ model leads to a structure factor $S(Q, \omega, T=0)$, which 
vanishes for $\omega \leq \Delta_{v}$, as a consequence of the finite activation gap $\Delta_{v}$ of the pair of bound $Z_{2}$ fluxes (or "visons") created by the application of a spin operator to the ground state.

Despite these qualitative differences between the low-energy magnetic response function of the quantum and the classical Kitaev liquids at $T=0$, we will show in this manuscript that the magnetic structure factors, $S(Q, \omega, T)$, of both models become very similar for $\omega$ and $T$ bigger than a quantum to classical crossover energy scale $T_{Q C}$. Our results then suggest the possibility of describing the thermally induced random-flux state at $T \gtrsim \Delta_{v}$ in the $S=1 / 2$ Kitaev model $[151,152,160]$ with the classical liquid of the model obtained in the $S \rightarrow \infty$ limit. This observation can be exploited to identify proximate quantum Kitaev liquid materials because $S(Q, \omega, T)$ can be computed under control for any arbitrary deformation of the pure Kitaev Hamiltonian in the classical limit.

\subsection{Zero-Temperature Liquids}

The dynamics of the classical version of Kitaev model is studied by combining Metropolis sampling and Landau-Lifshitz (LL) dynamics:

$$
\frac{d S_{i}}{d t}=S_{i} \times B_{i}
$$

where $B_{i}$ is the effective local field (molecular field) acting on the spin $S_{i}$. The temperature of the simulation is fixed during the Metropolis sampling and, the Landau-Lifshitz dynamics starts from a randomly selected well thermalized configuration. To study the effect of relevant perturbations, which replace the Kitaev liquid by an ordered state at lowenough temperatures, we will consider the simple case of the classical Kitaev-Heisenberg (KH) Hamiltonian $[157,158]$ with only nearest-neighbor (NN) interactions:

$$
H=K \sum_{\nu=x, y, z} \sum_{\{i, j\}_{\mathcal{V}}} S_{i}^{\nu} S_{j}^{v}+J \sum_{\{i, j\}} S_{i} \cdot S_{j}
$$

The index $v$ for the variable $i, j$ indicates that the two n.n. sites $i$ and $j$ are connected by a $v v$ bond [see Figure 8.2(a)]. To compare the results of this classical model against the spin $S$ quantum version of the model, we normalize the classical spins as $\left|S_{i}\right|=\sqrt{S(S+1)}$. The quantum version of this Hamiltonian has been proposed as a model for iridium or 
ruthenium-based materials $[139,161--163]$. The magnetic structure factor $S(Q, \omega, T)$ is obtained by Fourier transforming the real-space correlator $\left\langle S\left(r_{i}, t\right) . S\left(r_{0}, 0\right)\right\rangle$ evaluated from the LL dynamics over a finite period with periodic boundary conditions.
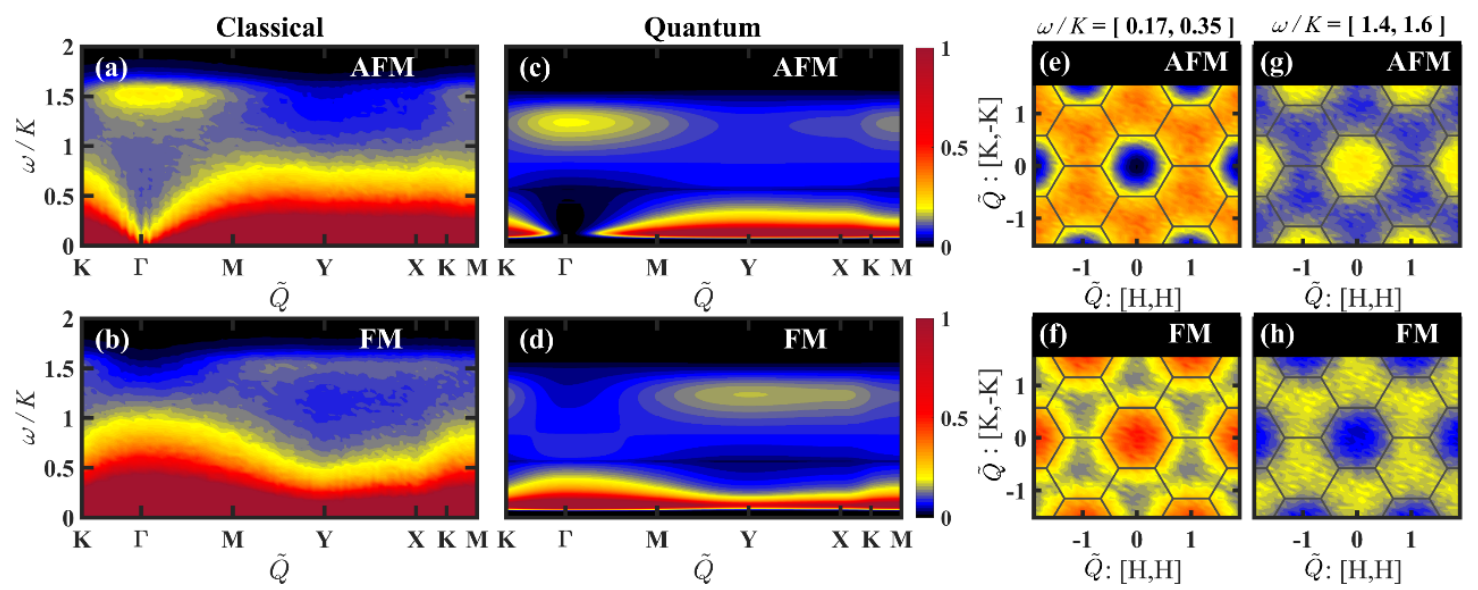

Figure 8.2 Comparison of the dynamical structure factor, $S(Q, \omega)$

$S(Q, \omega)$ in the classical limit $(S \rightarrow \infty)$ and quantum limit $(S=1 / 2)$ of the pure Kitaev model $(J=0)$ at $T=0$. Panels (a) and (b) show $S(\mathrm{Q}, \omega)$ obtained from LL simulations of the classical AFM and FM Kitaev models, respectively. Panels (c) and (d) show $S(Q, \omega)$ at $T=0$ for the $S=$ 1/ 2 Kitaev model obtained in Ref. [144] . Constant energy cuts of (e) AFM and (f) FM classical Kitaev liquids obtained by integrating over the energy range $\omega /|K|=[0.17,0.35]$, corresponding to the low-frequency mode. Similar plots for the high-frequency mode are also shown for (g) AFM and (h) FM classical Kitaev liquids with the integration energy range $\omega /|K|=[1.4,1.6]$.

We first focus on the pure Kitaev limit $(J=0)$. Figure 8.2 shows the magnetic structure factor $S(Q, \omega)$ of the classical and the quantum models at $T=0$ for both antiferromagnetic (AFM) and ferromagnetic (FM) cases. $S(Q, \omega)$ is plotted along the BZ path (KГMYXK) shown in Figure 8.1(b). The calculations in the classical limit (CL), shown in Figure 8.2 (a) and (b), are averages over 120 LL simulations on a supercell of $20 \times 20$ unit cells (800 spins). The quantum limit (QL) calculations, shown in Figure 8.2 (c) and (d), correspond to the exact result in the thermodynamic limit [6]. Remarkably, both the classical and the quantum Kitaev liquids are found to have two different almost dispersionless modes centered at high and low frequencies $(\omega)$ with striking similarities.

The high-energy mode is centered around the $\boldsymbol{\Gamma}(\mathbf{Y})$ point for $K>0(K<0)$ and it is accompanied by a suppression of the low-energy spectral weight centered around the same wave vector. This behavior is better illustrated by the contour plots shown in Figure 8.2 
(e)-(h). These panels are constant frequency cuts of $S(Q, \omega)$, which show the distribution of spectral weight over momentum space. Figure 8.2 (e) and (f) correspond to the distribution of low-frequency modes (integral of $S(Q, \omega)$ over the interval $\omega /|K|=[0.17$, 0.35]), while Figure $8.2(\mathrm{~g})$ and $2(\mathrm{~h})$ show the distribution of high-frequency modes (integral of $S(Q, \omega)$ over the interval $\omega /|K|=[1.4,1.6]$ ). As it is clear from these panels, the low-energy spectral weight is suppressed in the same region in momentum space where the distribution of high-energy spectral weight has a peak. This is the center of the first BZ for $K>0$ and the center of the second BZ for $K<0$ [see Figure 8.1 (b)].
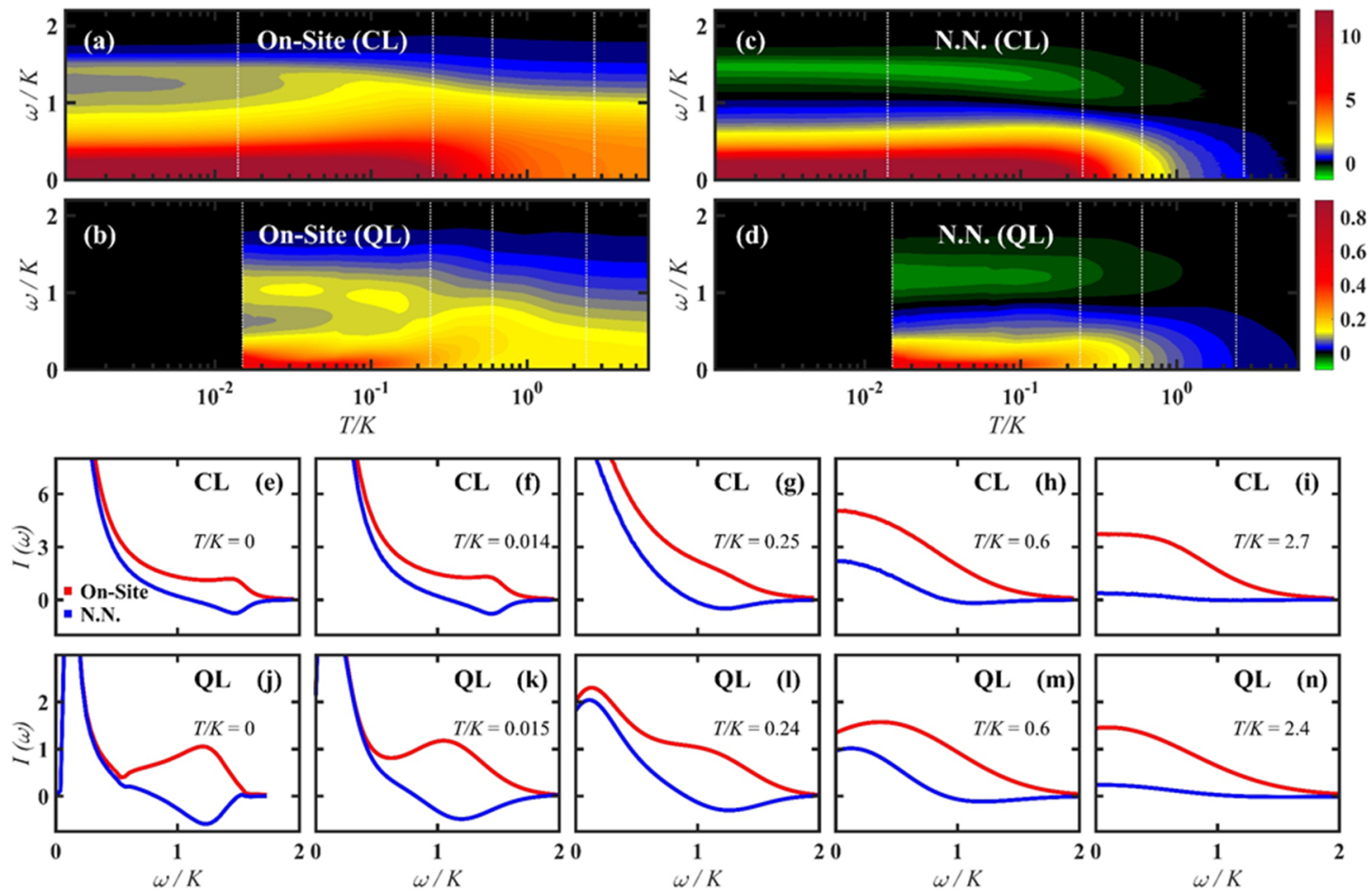

Figure 8.3 Temperature dependences of spin-spin correlations.

Temperature dependences of the dynamical spin structure factor decomposed into the on-site contribution, $S_{0}(\omega)$, and the NN contribution, $-S_{1}(\omega)$, for the classical Kitaev model and also for the $S=1 / 2$ Kitaev model: $S_{0}(\omega)$ for (a) $S=\infty$ and (b) $S=1 / 2$ and $-S_{1}(\omega)$ for (c) $S=\infty$ and (d) $S=1 / 2$. Frequency-dependence of $S_{0}(\omega)$ and $-S_{1}(\omega)$ in the classical model at selected temperatures (e) $T / K=0$, (f) 0.014, (g) 0.25, (h) 0.6 and (i) 2.7 [indicated by the dashed lines in panels (a) and (c)]. Similar plots for the $S=1 / 2$ model are shown for comparison for (j) $T / K=0$, (k) 0.015, (m) 0.24, (l) 0.6 and (n) 2.4 [indicated by the dashed lines in panels (c) and (d)]. Note that the n.n. dynamical correlation function is $\pm S_{1}(\omega)$ for $K= \pm|K|$, while the onsite correlator is the same for both signs of $\mathrm{K}$.

To understand the differences and similarities between the classical and the quantum limits of the Kitaev model, it is instructive to go back to the real-space. Figure 8.3 shows the real 
space spin-spin correlators for the classical and the quantum limits of the AFM model. Figure 8.3 (a) and (b) include the on-site correlator for the CL and the QL, respectively. Similarly, Figure 8.3 (c) and (d) contain the NN correlator for the CL and the QL, respectively. As we mentioned before, the local gauge structure shared by the quantum and the classical models leads to a real space spin-spin correlator that vanishes beyond NN sites. This implies that the spin structure factor in the pure Kitaev model for arbitrary S can be decomposed as

$$
S^{v v}(Q, \omega)=S_{0}(\omega)+\cos \left(Q \cdot \mathrm{u}_{v}\right) S_{1}(\omega)
$$

where $v=x, y, z$ and $\mathrm{u}_{v}$ is the relative vector between two NN sites connected by a $v v$ bond; $S_{0}(\omega)$ and $S_{1}(\omega)$ are the Fourier transformations into the frequency domain for the on-site and the NN dynamical spin correlators, respectively. This peculiarity leads to the sinusoidal Q-modulation in the high- and low-energy peak intensities as illustrated in Figure 8.2 (e)-(h). In other words, the similar wave vector dependence of the different modes in the classical and the quantum limits are a direct consequence of the similar real space correlations shown in Figure 8.3.

The real space correlators also exhibit a low and a high-frequency peak in both models. The low-frequency peak of the $S=1 / 2$ model appears right above the small activation gap $\Delta_{v}$ for the pair of excited $\mathrm{Z}_{2}$ gauge fluxes (visons) created by the action of a spin operator on the ground state. The extended linewidths of the low and the high-energy peaks arise from the continuum of Majorana fermion excitations, which leads to a rather narrow low-energy peak and a broad high-energy peak. In contrast, the low energy peak of the classical model extends down to zero frequency because the fluxes become gapless in this limit. The $\mathrm{CN}$-ground states are not eigenstates of the $W_{p}$ operators, implying that the classical limit $(S \rightarrow \infty)$ corresponds to a flux condensation (the flux number is no longer a good quantum number). It is interesting to note that quadratic quantum fluctuations partially restore this quantum number by selecting the $\mathrm{CN}$-ground states, which maximize the number of hexagonal plaquettes with well-defined flux equal to zero (eigenvalue of $W_{p}$ equal to one). These are the $3 \times 2^{N / 3}$ states with every single loop being an elementary hexagon [154]. The extensive degeneracy of the classical limit leads to sharp $\delta$-function 
singularity in the spectral weight at $\omega=0$. The simple analysis that we present below explains the origin of this singularity and of the high-energy peak centered around the $\Gamma$ point of the AFM model [see Figure 8.2 (a)].

The main qualitative aspects of the dynamical structure factor of the classical model at $T=$ 0 can be captured by the CN-ground states. As explained in Ref. [154], the CN-ground states can be mapped into the close-packed dimer coverings of the honeycomb lattice by assigning one dimer to each "satisfied" bond (i.e., with its local energy taking the minimum value) [see Figure 8.1 (c)]. Within linear spin-wave theory [154], magnons for CN-ground states can only propagate along the one-dimensional paths of empty bonds because of the Ising nature of the interactions. These 1D paths become self-avoiding loops if we adopt closed boundary conditions, which fully cover the whole lattice (every spin site is visited by one and only one loop). The spin-wave Hamiltonian for each loop is invariant under translations by two sites along the loop [154]. In other words, the unit cell of the loops has two sites, implying that each loop has two branches of magnetic modes: a flat branch of zero modes, $E_{0}(k)=0$, and second branch with a dispersion relation

$$
E(k)=2|K| S \cos (k / 2)
$$

with $k$ being the momentum associated with the two-unit translation within a loop.

The top of the single-magnon band is at $k=0$, implying that the density of single-magnon states has a Van Hove singularity at $k=0$ for infinitely long loops. For the AFM model $(K>0)$, the $k=0$ magnon wave function has the same phase for both sites in the unit cell. Consequently, the singular density of states leads to a high-energy peak centered at the $\Gamma$ point [Figure 8.2 (a)]. On the other hand, the flat band of zero modes leads to a deltalike contribution at $\omega=0$. While the real space dynamical structure factors are obtained by averaging over all the $\mathrm{CN}$-ground states, this average is dominated by loops of very long length because of the critical nature of the close-packed dimer coverings of the honeycomb lattice. (The fully-packed self-avoiding loops on the honeycomb lattice is a critical system that can host loops of in finite length [164,165].) Consequently, we can 
approximate the average over loops by the result that is obtained for an infinitely long loop (see Appendix F ):

$$
S_{0}(\omega) \simeq S \pi \frac{\left(\frac{\omega}{K S}\right)}{\omega}+\frac{\pi S}{2} \delta(\omega) \int_{-\pi}^{\pi} \frac{d k}{\cos \left(\frac{k}{2}\right)}
$$

for the average on-site spin-spin correlator, and

$$
\theta S_{1}(\omega) \simeq\left[\frac{\pi \omega S}{2(K S)^{2}}-\frac{\pi S}{\omega}\right] \bar{\rho}\left(\frac{\omega}{K S}\right)-\frac{\pi S}{2} \delta(\omega) \int_{-\pi}^{\pi} \frac{d k}{\cos \left(\frac{k}{2}\right)}
$$

for the NN spin-spin correlator, where $\theta=K /|K|$ is the sign of $K$. The function $\bar{\rho}(x)=$ $K S \rho(x)$ is the dimensionless density of single magnon states,

$$
\bar{\rho}(x)=\frac{2}{\sqrt{1-\left(\frac{x}{2}\right)^{2}}}
$$

The 1/ $\omega$ singularity that appears in the first term of Equations 8.7 and 8.8 arises from the 1D nature of the problem at the linear spin-wave level. This singularity must then be regularized by higher order corrections in the $1 / S$ expansion, which restore the 2D nature of the problem by connecting different loops. Nevertheless, we will see that the linear spinwave contributions Equation 8.7 and Equation 8.8 are already enough to understand the main features of the numerical results. In particular, the $1 / \omega$ tail explains the broad lowenergy spectral weight of the numerical results shown in Figure 8.2 (a) and (b).

The second term of Equations 8.7 and 8.8 correspond to the singular contribution from the flat band of zero modes in each loop. We note that these singular contributions to the onsite and the $\mathrm{NN}$ correlation functions differ only by a minus sign. Equation 8.5 then implies that the singular contribution from the zero modes vanishes exactly at the $\Gamma$ point for the AFM classical Kitaev model: $S^{\nu v}(\Gamma, \omega)=S 0(\omega)+S 1(\omega)$. This fact remains true for any loop length. In other words, the distribution of zero modes over the Brillouin zone (BZ) has a node at the $\Gamma$ point for $K>0$. Moreover, the singular contributions from the first terms of Equations 8.7 and 8.8 (infrared singularity associated with the 1D nature of the loops) also cancel exactly at the $\Gamma$ point. The numerical result for this distribution is shown in Figure 8.2 (e) for $K>0$ and in 2(f) for $K<0$. As expected from our analysis, $S(Q, \omega \rightarrow$ 
0 ) is suppressed around the $\Gamma$ point for $K>0$. This "hole" in the density of zero modes is a signature of the AFM classical Kitaev liquid. Similarly, the FM Kitaev liquid is characterized by a suppression of the density of zero modes around the Y point (center of the second BZ). In this case, the zero-mode contribution cancels exactly at the Y point only for the component $S^{\nu v}(Y, \omega)=S_{0}(\omega)-S_{1}(\omega)$ with the $v v$ bond is parallel to $\mathrm{Y}$. Consequently, the singular weight contribution at $\omega \rightarrow 0$ is suppressed at the $\mathrm{Y}$ point, but it does not vanish. In both cases, the missing low-energy spectral weight is shifted to the high-energy peak at $\omega \rightarrow|K|$, as it is shown in Figure $8.2(\mathrm{~g})$ and $2(\mathrm{~h})$.
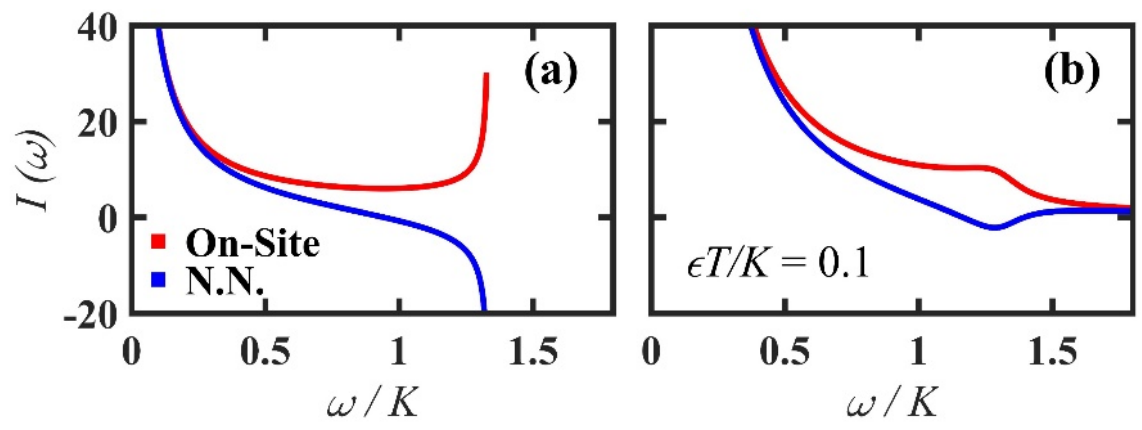

Figure 8.4 Spin-spin correlations of $\mathrm{CN}$-ground states.

(a) Frequency dependence of $S_{0}(\omega)$ and $-S_{1}(\omega)$ associated with the CN-ground states in the classical Kitaev model at $\mathrm{T}=0$. (b) Refined evaluations of $S_{0}(\omega)$ and $-S_{1}(\omega)$ with the inclusion of an artificial broadening $\epsilon=0.1 \mathrm{~K}$, which mimics the effect of the continuum of the intermediate ground states connecting different $\mathrm{CN}$-ground states through the slide transformations (see the text).

As we anticipated, the zero modes are removed in the quantum limit because the massive ground state degeneracy is lifted by quantum fluctuations. The net result is that the divergent spectral weight at $\omega=0$ is transferred to a small, but finite frequency region $\omega \gtrsim \Delta_{v}$. The lack of zero modes at the $\Gamma$ point suggests that the "hole" in the low-energy spectral weight should still be present in the spectral weight distribution right above the two-vison gap $\Delta_{v}$. This expectation is confirmed by the numerical results shown in Figure 8.2 (a) for $K>0$ and Figure 8.2 (b) for $K<0$. Consequently, this suppression of the low energy spectral weight and the associated shift to high energy is also a characteristic of the quantum Kitaev liquid. We note that low-energy spectral weight around the $\Gamma$ point can in principle be induced by perturbations that break the two-flux selection rule (spin operators connecting subspaces that differ by two-gauge fluxes). However, as it was shown 
in Ref. [166], the rule is broken to fourth order in the typical perturbations of the Kitaev Hamiltonian, implying that the low-frequency spectral should remain very small.

Figure 8.4 (a) shows the on-site, $S_{0}(\omega)$, and the NN, $S_{1}(\omega)$, dynamical structure factors given by Equations 8.7 and 8.8, respectively. These quantities are only an approximation of the exact $S(Q, \omega, T=0)$ because the average is taken over the $\mathrm{CN}$-ground states. The missing ground states correspond to the continuous deformations that connect different CN-ground states [154].

Let us consider an intermediate ground state, which is obtained by continuous "slide" transformations [154] of a given $\mathrm{CN}$-ground state. A slide transformation only involves spins along either a closed loop or an infinitely long string corresponding to alternating dimer and empty bonds. For small transformations, magnons will still propagate mainly along the loops of the "parent" CN-state. However, the corresponding 1D spin-wave Hamiltonian is no longer translationally invariant. Moreover, magnons can tunnel between different loops because the spins on the "satisfied bonds" are no longer parallel to the Ising anisotropy axis of the bond. In other words, the spin-wave Hamiltonian of the intermediate state can be regarded as a disordered version of the spin-wave Hamiltonian of the parent $\mathrm{CN}$-state. To zeroth order, the effect of disorder is to broaden the quasi-particle peaks of the parent CN-state. Figure $8.4(\mathrm{~b})$ shows $S_{0}(\omega)$ and $S_{1}(\omega)$ after introducing an effective broadening $\epsilon=0.1 \mathrm{~K}$. These curves reproduce quite well the numerical results shown in Figure 8.3 (e). In particular, the effective disorder introduced by the "intermediate" ground states broaden the high-frequency peak originated by the Van Hove singularity in the density of states. The success of such a minimal perturbative treatment of intermediate states relies on the fact that $\mathrm{CN}$-ground states maximize the number of zero modes, i.e., most of the classical ground states are small deformations of $\mathrm{CN}$-ground states [154]. Although this property does not lead to an order-by-disorder phenomenon at any temperature, it may renormalize the effective stiffness leading to a nontrivial $T$ - dependent power law in the short-range decay of the energy density correlator [167].

Finally, 1D magnons must decay into pairs of spinons upon inclusion of quantum fluctuations beyond linear spin-wave theory. The resulting two-spinon continuum can be regarded as a precursor of the Majorana modes, which appear in the $S=1 / 2$ limit. The 
main effect of the two-spinon continuum is to broaden the high-frequency peak, in agreement with the result obtained for the quantum limit of the model [see Figure 8.3(b), (d) and (j)].

Thus, to summarize our discussion on the low- $T$ classical liquid state, the high-energy peak of $S(Q, \omega)$ is common to the quantum $(S=1 / 2)$ and the classical $(S \rightarrow \infty) T=0$ liquids. The classical limit of the model provides a new insight for understanding the origin of this peak, which has been used as a fingerprint of the proximity to a Kitaev liquid state $[140,148,149]$. The classical model also provides a new insight for understanding the momentum dependence of the low-energy spectral weight distribution of the $S=1 / 2$ model. This distribution is very similar to the distribution of the $\omega=0$ spectral weight induced by the zero modes of the classical model. These facts establish a clear connection between the $T=0$ spectra of the classical and quantum liquids.

\subsection{Finite Temperature Liquids}

The $S=1 / 2$ Kitaev model can be mapped into a gas of free Majorana fermions interacting with static $Z_{2}$ gauge fields [138]. Because of the quadratic nature of the action, the fermionic degrees of freedom can be integrated out to obtain an effective classical action for the $Z_{2}$ variables, which can be simulated by using Monte Carlo simulations [151,152] , similar to other problems of noninteracting fermions coupled to classical degrees of freedom [168]. To evaluate the dynamical spin structure factor at finite temperatures, one needs to combine such Monte Carlo samplings with a quantum Monte Carlo solver [147] . To compare against the results for $S(Q, \omega, T)$ of the $S=1 / 2 \mathrm{Kitaev}$ model [see Figure 8.3], extracted from Refs. [145] and [146], we present the corresponding results for the classical Kitaev model in Figure 8.3 and Figure 8.5.

As shown in Figure 8.5 (d) and (h), the high-temperature paramagnetic (PM) state of both the AFM and FM classical Kitaev models exhibits a characteristic broad diffusive peak with a small $Q$-dependence. Figure 8.5 (c), (g), (b), and (f), show that the low-frequency diffusive mode becomes more structured upon decreasing temperature and an additional mode emerges at the $\mathrm{BZ}$ center for $K>0$ and at the center of the second BZ (Y point) for 
$K<0$. Finally, at very low temperatures [see Figure 8.5 (a) and (e)] the upper mode goes up in energy and it separates from the low-frequency spectral weight.
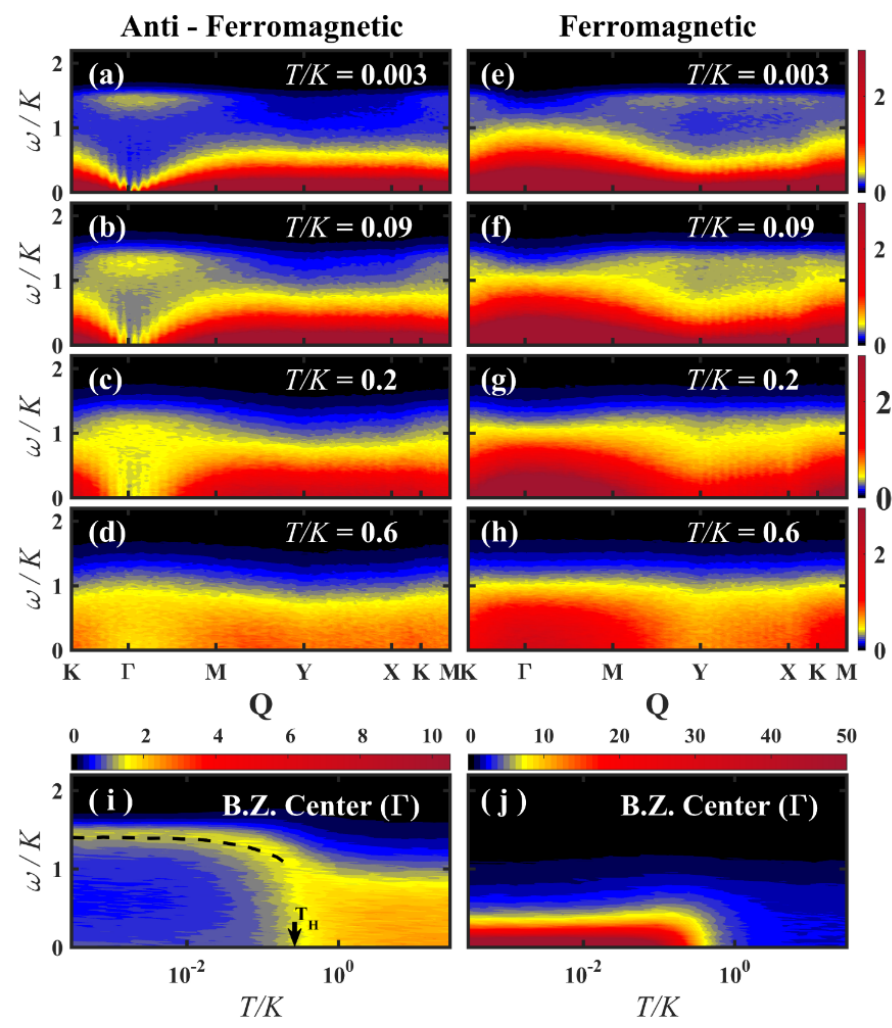

Figure 8.5 Temperature evolution of $S(Q, \omega)$ of AFM and FM Kitaev models.

Temperature evolution of $S(\mathrm{Q}, \omega)$ in the classical Kitaev model calculated along a BZ path shown in Fig. 1 (b) for the AFM coupling [(a)-(d)] and the FM coupling [(e)-(h)] at (a),(e) $T / K=$ 0.04, (b),(f) $T / K=1.1$, (c),(g) $T / K=2.9$ and (d),(h) $T / K=7.5$. Panels (i) and (j) show the temperature dependence of the intensity at the $\Gamma$-point for the AFM and the FM models, respectively. The dashed line in (i) traces the peak position of the high energy mode.

Figure 8.5 (i) and (j) show the temperature dependence of $S(\Gamma, \omega)$ for the AFM and the FM cases, respectively. The dashed line in Figure 8.5 (i) indicates the temperature evolution of the high-frequency peak. This peak merge with the low-energy peak at a temperature scale $T_{H} \sim 0.3-0.4 \mathrm{~K}$, which roughly coincides with the high-temperature peak of the specific heat curve of the quantum $(S=1 / 2)$ version of the model [169]. $T_{H}$ is also the temperature at which the high-energy mode of the $S=1 / 2 \mathrm{Kitaev}$ model merges with the low-energy mode $[145,146]$.

The quantum to classical crossover occurs at the temperature scale $T_{Q C} \simeq \Delta_{v}$, which is significantly lower than TH. Degenerate Hamiltonian eigenstates whose number of fluxes 
differ by two only exist for energies bigger than $\Delta_{v}$, as it is indicated by the finite value of $S_{0}(\omega \rightarrow 0)$ for the $S=1 / 2$ Kitaev model [see Figure 8.3 (1)]. Linear combinations of these degenerate states produce eigenstates with non-zero $\left\langle S_{j}\right\rangle$ (the total flux is no longer a good quantum number), which can be regarded as "classical states".

A large concentration of $Z_{2}$ fluxes is induced at $T>T_{Q C} \simeq \Delta_{v}$. These fluxes act as scattering centers for the Majorana fermions, which lose their coherence when the distance between scattering centers becomes comparable to their wave-length. This condition is fulfilled at $T>T_{Q C}$ because the average distance between thermally activated fluxes becomes of order one lattice space. Figure $8.3(\mathrm{~g})$ and (l) show that the dynamical structure factors of the quantum and classical AFM models are very similar for $T / K=0.25$ and $\omega>\Delta_{v}$. The quantum character of the liquid is manifested at low temperatures in the lowfrequency dip at $\omega<\Delta_{v}$ visible even up to $T / K=0.6 \mathrm{~K}$ (Figure $8.3(\mathrm{~m})$ ). The resulting low-frequency peak is then a remnant of the 2-vison gap.

\subsection{Kitaev-Heisenberg Model}

A big advantage of the classical limit of the model is that we can study the evolution of $S(Q, \omega)$ away from the Kitaev point. In contrast to the quantum case, an arbitrarily small perturbation is enough to replace the $T=0$ liquid with a magnetically ordered phase that is also stable at finite temperatures. This ordered phase has three different regimes: a low temperature regime, $T \ll T_{N}$, in which the magnetic structure factor is dominated by the single-particle excitations of the ordered state (spinwave dispersion), an intermediate temperature regime, $T_{N}<T \lesssim K$, with liquid-like correlations, and a high-temperature paramagnetic state, $T \gg K$, that can be regarded as a "spin-gas" because the on-site correlations are negligibly small in comparison to the on-site correlations. Price and Perkins [157,158] argued that the ordering occurs via two consecutive BerezinskiiKosterlitz-Thouless transitions, as in the $C_{6}$ clock model, with a small critical phase in between.

The KH model (Equation 8.4) is one of the simplest Hamiltonians that can be used to study the three regimes (in what follows, our discussion excludes details related to critical phenomena at $T \approx T_{N}$ ). Given that the intermediate spin-liquid regime only exists in the 
proximity of a $T=0$ liquid phase (see Figure 8.6), this regime can be used to detect proximate quantum spin liquid behavior. If $T_{N}>T_{Q C}$, the liquid-like regime is described by the classical limit of the model, implying that finite-T classical spin dynamics can be exploited to identify magnets near a $T=0$ quantum melting point.

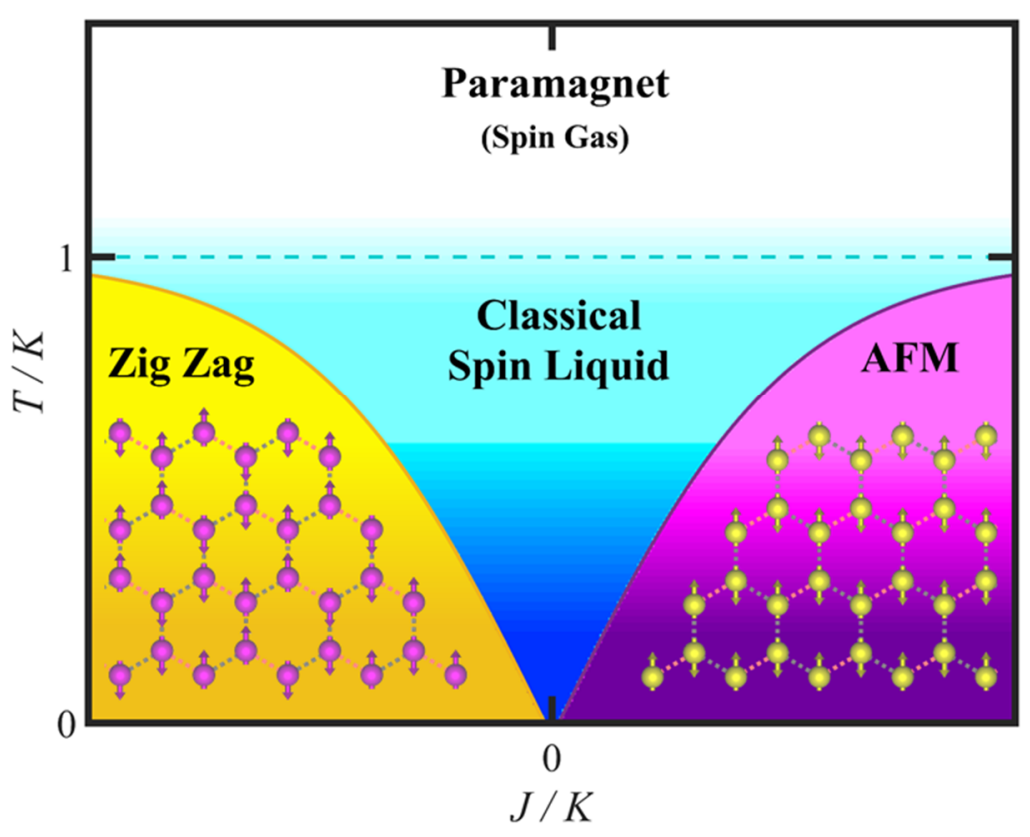

Figure 8.6 Schematic phase diagram of the classical K-H model for $(K>0)$

The color gradient denotes the quantum-classical crossover region. The solid lines represent phase transitions. We note that the quantum spin liquid phase is stable over a finite interval of $J / K$ values around $J=0$ in the quantum limit $(S=1 / 2)$.

As shown in Figure 8.6, the ground state of the classical $\mathrm{KH}$ model has zig-zag ordering for $J / K<0$ and a two-sub-lattice AFM ordering for $J / K>0(K>0)$. At $T=0$, the spinwave dispersion deviates from the linear spin-wave spectrum (green lines in Figure 8.7) upon approaching $\mid \mathrm{Jl} / K=0$. These deviations arise from the non-linear effects associated with spin fluctuations towards the large manifold of classical states that become ground states for $J=0$. We note that this nonlinearity may have a different manifestation in the quantum $S=1 / 2$ model. In particular, the magnon modes of the $S=1 / 2$ model should become weakly bounded pairs of Majorana fermions upon approaching the transition into the spin liquid phase (here we are assuming that the transition is continuous or quasicontinuous). It is clear that this intrinsically quantum phenomenon cannot be captured by the classical limit of the model. However, as we discuss below, the classical model is still 
capable of capturing the evolution of the high-energy features of the magnon spectrum. Moreover, based on the results discussed in the previous sections, the classical model can describe the second way of approaching the spin liquid regime, which is by increasing temperature at a fixed value of $J / K$.

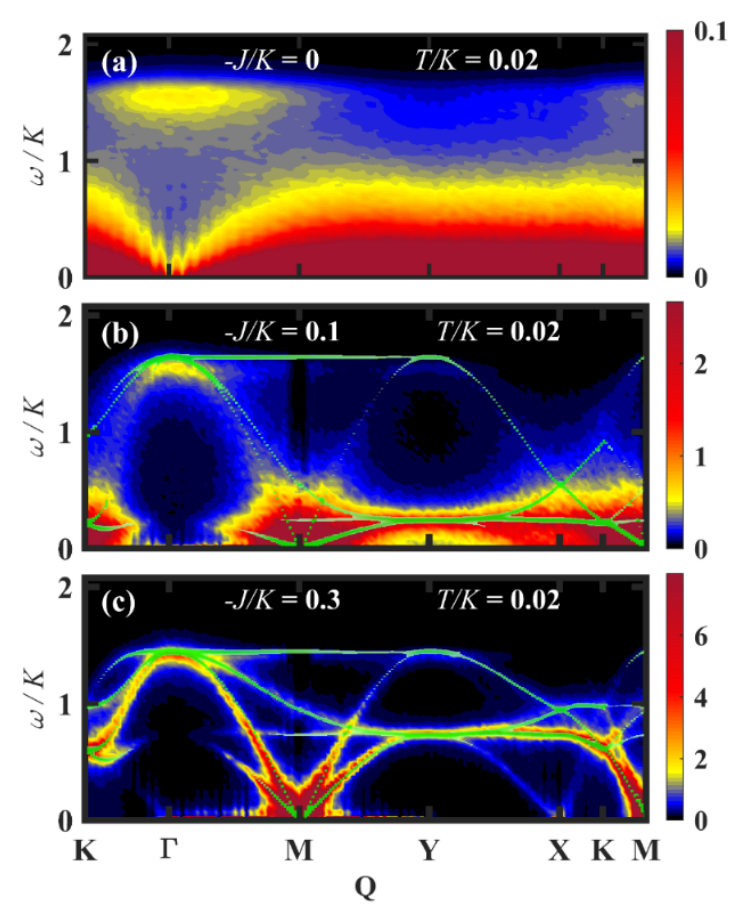

Figure 8.7 $S(Q, \omega)$ as a function of FM Heisenberg exchange $(J<0)$

$S(\mathbf{Q}, \omega)$ for the KH model with FM Heisenberg exchange $(J<0)$ and AFM Kitaev interaction $(K>0)$ as a function of $-J / K$. The green lines correspond to the $S(\mathbf{Q}, \omega)$ obtained from linear spin-wave theory (the line thickness indicates the intensity). Panel (a) corresponds to the pure Kitaev model $(J=0)$, while panels (b) and (c) correspond to $J / K=-0.1$ and $J / K=-0.3$, respectively. Panels (c) shows that the magnon modes are more sharply defined away from the Kitaev point. Panel (b) clearly shows that the magnon modes become less defined upon approaching the Kitaev point because of the increasing importance of non-linear effects captured by the LL simulation.

Figure 8.7 shows the evolution of the low-temperature $S(Q, \omega)$ as a function of $J / K$, from the pure AFM classical Kitaev model [Fig. 7(a)] to $-J / K=0.3$ [Figure 8.7(c)]. As expected, $S(Q, \omega)$ exhibits a sharply defined spin wave dispersion with a pseudoGoldstone mode at the M-point (ordering wave vector) well inside the ZZ phase. We note that there are three inequivalent M-points corresponding to the possible directions of the FM ZZ chains. Upon reducing $|J| / K$, the magnon modes become less defined due to the 
increasing relevance of non-linear effects triggered by the proximity to the $(J=0)$ spin liquid point. In particular, the spectrum obtained for $J / K=-0.1$ [Figure 8.7(b)] shows an overall softening of the acoustic magnon modes, except for the region around the $\Gamma$ point where the spectral weight remains around the original optical mode. The evolution of this "high-energy" feature should be common to both the classical and quantum limits of the model. In other words, this unusual behavior can be used to detect the proximity to a Kitaev quantum spin liquid, as long as the transition between the $\mathrm{ZZ}$ phase and the liquid state remains continuous (or quasi-continuous) in the $S=1 / 2$ limit.

As shown in Figure 8.6, the spin liquid state can also be accessed by increasing $T$ at a fixed value of $J / K$. Figure 8.8 shows the temperature evolution of $S(Q, \omega)$ for $J / K=-0.1$. The corresponding Néel temperature is $T_{N} \approx 0.06 \mathrm{~K}$, implying that there is a temperature window, $|J|<T<K$, above $T_{N}$, where $S(Q, \omega, T)$ should be very similar to the dynamical structure factor of the pure Kitaev model ( $T$ is high-enough to suppress the magnetic correlations induced by the Heisenberg interaction). This is true for any other small perturbation that can be added to the pure Kitaev model. Figure 8.8 (c) confirms this expectation: $S(Q, \omega)$ is very similar to the result shown in Figure 8.5 (c) for the pure Kitaev model $(J=0)$. As shown in Figure $8.8(\mathrm{~g})$, the low frequency region shows the signature of a Kitaev liquid, with a rather uniform distribution of low energy modes over the BZ, which is suppressed around the $\Gamma$ point. Correspondingly, Figure $8.8(\mathrm{k})$ shows the opposite behavior for the distribution of high-energy modes over the BZ $(1.2<\omega / K<1.6)$. The rest of the panels on the right-hand side of Figure 8.8 show the continuous redistribution of spectral weight upon moving towards the low and high temperature regimes. Given that quantum corrections are small above $T_{Q C} \simeq \Delta_{v} \simeq 0.06 \mathrm{~K}$, the result obtained at $\omega>\Delta_{v}$ with the classical spin model represents the $S(Q, \omega, T)$ of the QL (see Figure 8.3).

We remark that the Kitaev liquid state that appears in the intermediate temperature regime is independent of the particular model Hamiltonian, as long as the additional terms can be treated as small perturbations relative to the Kitaev contribution (separation of energy scales). This observation is relevant for candidate materials based on $4 d$ and $5 d$ elements because their microscopic Hamiltonian models include multiple interaction terms, whose values are still uncertain [159,170--182]. 


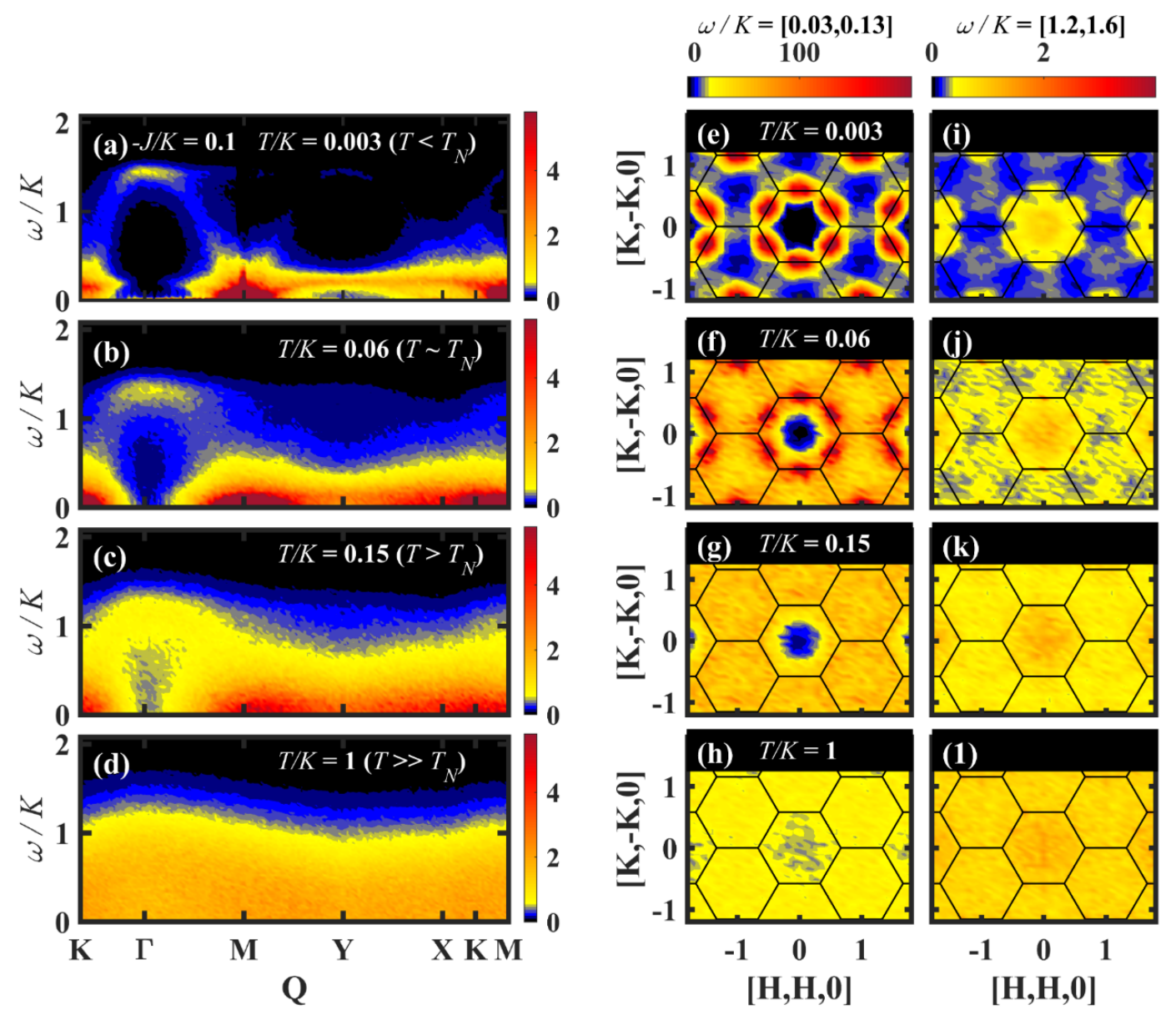

Figure 8.8 $S(\mathrm{Q}, \omega)$ obtained for the $\mathrm{KH}$ model with $J / K=\mathbf{- 0 . 1}$

The different panels show $S(\mathbf{Q}, \omega)$ along the BZ path KГMYХKM for (a) $T / K=0.02(T \ll$ $\left.T_{N}\right)$, (b) $T / K=0.09\left(T \simeq T_{N}\right)$, (c) $T / K=0.17\left(T>T_{N}\right)$ and $T / K=1.7\left(T \gg T_{N}\right)$. The panels on the right-hand side show the distribution of spectral weight over the BZ: the first column shows the low-energy spectral weight $(0.03<\omega<0.13)$, while the second column shows the spectral weight in the frequency interval of the high-energy peak $(1.2<\omega<1.6)$. 


\subsection{Conclusions}

We have shown that the dynamical spin structure factors of the classical and the quantum $(S=1 / 2)$ limits of the Kitaev model become very similar above a crossover temperature $T_{Q C} \simeq \Delta_{v}$. Moreover, both structure factors exhibit similar qualitative behavior in their high-frequency response $(\omega \gtrsim K)$ even at $T=0$. This "high-energy" response is characterized by a broad peak above $\omega \simeq K$, which is centered around the $\Gamma$ point for AFM Kitaev model $(K>0)$ and around the $\mathrm{Y}$ point for the FM Kitaev model $(K<0)$. Correspondingly, the singular spectral weight at $\omega=0$, produced by the zero modes of the classical model, is suppressed around the $\Gamma$ point for AFM Kitaev model and around the $Y$ point for the FM Kitaev model. This dip in the momentum space dependence of the lowenergy spectral weight is still present in the $S=1 / 2$ model. The main difference is that the low-energy modes of the quantum $S=1 / 2 \mathrm{Kitaev}$ model appear right above the twovison activation gap $\Delta_{v}$.

In the classical limit, the low-energy modes of the $\mathrm{CN}$-ground states correspond to singlemagnons states that propagate in 1D loops [154]. The high-energy peak of the classical Kitaev model arises from the singular density of single-magnon states at the top of the dispersive branch of excitations. This Van Hove singularity is smoothed out by the deformations of the $\mathrm{CN}$-ground states into the non-Cartesian ground states (valleys) that lead to the at branch of zero modes and also by thermal fluctuations. As discussed in Ref. 16 , the $1 \mathrm{D}$ magnons of the $\mathrm{CN}$-ground states must decay into fractionalized excitations upon inclusion of quantum fluctuations. These excitations can be regarded as precursors of the Majorana modes obtained in the quantum $S=1 / 2$ limit. In particular, this fractionalization leads to an additional broadening of the high-energy peak of $S(Q, \omega)$, which explains why the peak of the classical model is narrower than the peak of the $S=$ $1 / 2$ model.

Our results provide a systematic procedure for identifying the proximate quantum spin liquid behavior of real materials. A dip in the density of low-energy modes at the $\Gamma(\mathrm{Y})$ point must be accompanied by a high-energy peak around the same wave-vector for $K>$ $0(K<0)$. For materials that exhibit low-temperature magnetic ordering, the signatures of 
the Kitaev liquid should appear over an intermediate temperature window above the ordering temperature. As long as $T \gtrsim T_{Q C}$, the classical approach can be used in this temperature window to obtain a good approximation of $S(Q, \omega)$ for the $S=1 / 2$ model.

The analysis presented here can have more general implications for other quantum liquids with extensive ground state degeneracy in the classical limit. Given the lack of magnetic ordering, one needs to find an alternative low-energy characterization of the liquid state. Our results suggest that the distribution of zero modes over the BZ provides clear signature of the classical liquid, which is inherited by the distribution of low-energy modes of the quantum spin liquid. Given that such a distribution can be measured with inelastic neutron scattering, $[148,149,183]$ this experimental technique can play a crucial role in the characterization of quantum spin liquids. Moreover, the quantum to classical crossover can be exploited for computing other dynamical correlation functions and transport properties of quite general quantum spin models at $T>T_{Q C}$. 


\section{Appendix A Methods and Techniques}

\section{A.1 Synthesizing polycrystalline samples of $\operatorname{SCGO}(p)$ and $\operatorname{BCGO}(p)$}

Polycrystalline samples of $\operatorname{SCGO}(p)$ with twelve different values of $p=0.968(6), 0.960(9)$, 0.925(nominal), 0.917(9), 0.87(nominal), 0.844(8), 0.777(6), 0.736(6), 0.620(8), 0.5(nominal), 0.459(5) and 0.228(5) were made using standard solid-state synthesis techniques at the University of Virginia. Stoichiometric quantities of $\mathrm{SrCO}_{3}, \mathrm{Ga}_{2} \mathrm{O}_{3}$ and $\mathrm{Cr}_{2} \mathrm{O}_{3}$ were mixed, ground and pelletized. Then, the pellets were heated in air in dense $\mathrm{Al}_{2} \mathrm{O}_{3}$ crucibles at $1400{ }^{\circ} \mathrm{C}$ for $96 \mathrm{~h}$, with three intermediate grindings. The samples were then annealed at $1000^{\circ} \mathrm{C}$ for 7 days to promote cation ordering.

Polycrystalline samples of $\mathrm{BCGO}(\mathrm{p})$ with $\mathrm{p}=0.902(8), 0.806(6), 0.712(6), 0.635(7)$, 0.526(7) and 0.417(7) were made using standard solid-state synthesis techniques.10, 16) Stoichiometric quantities of $\mathrm{BaCO} 3, \mathrm{Ga} 2 \mathrm{O} 3$, and $\mathrm{Cr} 2 \mathrm{O} 3$ were mixed, ground and pelletized. The pellets were then heated in air in dense Al2O3 crucibles at $1400{ }^{\circ} \mathrm{C}$ for 96

$\mathrm{h}$, with three intermediate grindings. The samples were then annealed at $1000{ }^{\circ} \mathrm{C}$ for 7 days to promote cation ordering. 


\section{A.2 Bulk Susceptibility Measurements}

The DC magnetic susceptibility measurements on $\operatorname{SCGO}(p): p=0.620(8), 0.459(5)$ and 0.228 in the range of $0.5 \mathrm{~K}$ to $14 \mathrm{~K}$ for $\operatorname{SCGO}(p)$ is measured using the Quantum design MPMS-XL7 with a Helium-3 insert in the temperature range from $0.5 \mathrm{~K}$ to $14 \mathrm{~K}$ at ETH Zurich, while similar measurements were done on several other $\operatorname{SCGO}(p)$ samples with higher $p$ shown in Figure 4.2 in the temperature range of $2 \mathrm{~K}$ to $300 \mathrm{~K}$ at Kyoto University. The similar susceptibility measurements for $\operatorname{BCGO}(p): p=0.9,0.8,0.7,0.6,0.5$ and 0.4 samples has been measured using a Quantum Design Physical Property Measuring System (PPMS, Model 6000) with AC Measurement System (ACMS) option.
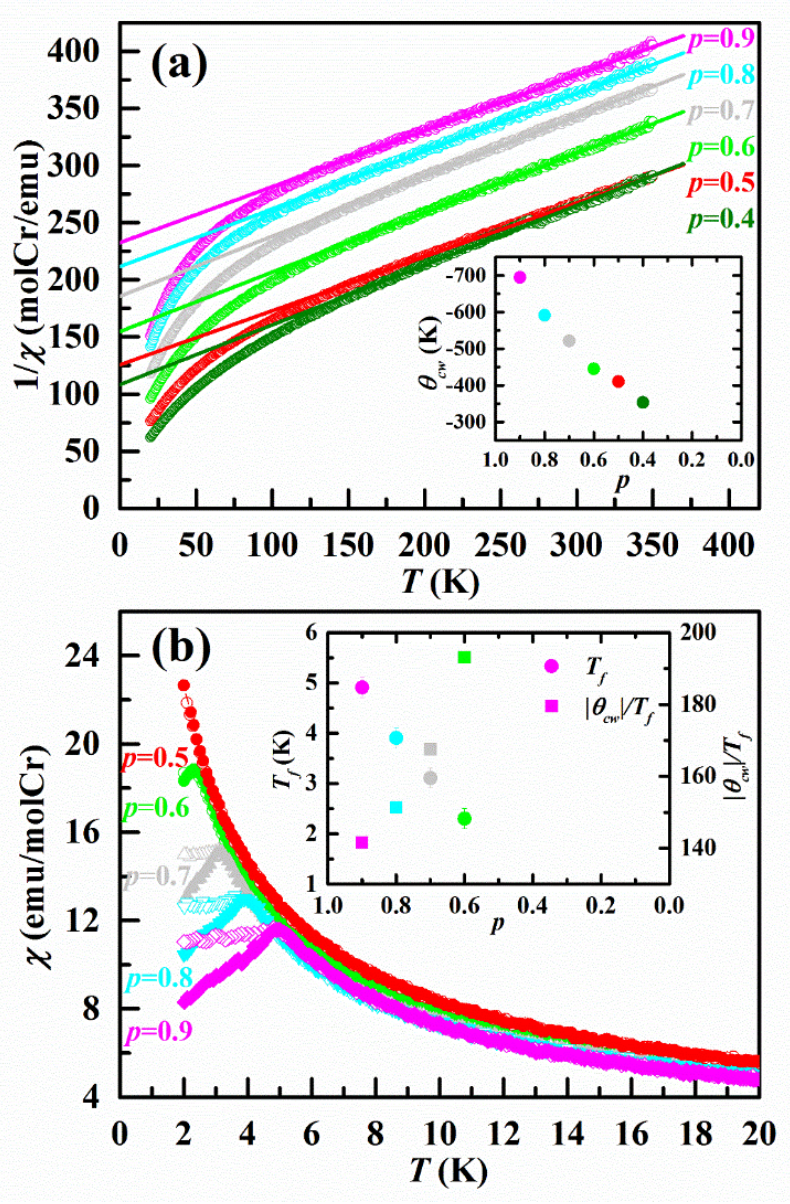

Figure (App.) A.1. Magnetic susceptibility measurements of $\operatorname{BCGO}(p)$

(a) Reciprocal bulk magnetic susceptibility of $\operatorname{BCGO}(p)$ with $p=0.902(8), 0.806(6), 0.712(6), 0.635(7)$, $0.526(7)$ and $0.417(7)$ in the temperature range from $20 \mathrm{~K}$ to $350 \mathrm{~K}, \mathrm{H}=0.1 \mathrm{~T}$. The straight lines are linear fits at $T>150 \mathrm{~K}$. The inset shows the $\theta_{c w}$ values obtained by Curie-Weiss fitting. (b) Bulk magnetic susceptibility of $\mathrm{BCGO}(p)$ with $p=0.902(8), 0.806(6), 0.712(6)$ and $0.635(7)$ in the temperature range from $2 \mathrm{~K}$ to $20 \mathrm{~K}: \mathrm{H}=$ 
$0.01 \mathrm{~T}$. Solid dots are field-cooled (FC) data and open dots are zero-field cooled (ZFC) data. The inset shows the freezing temperature $\left(T_{f}\right)$ and frustration index $\left|\theta_{c w}\right| / T_{f}$ as a function of $p$ value.

\section{A.3 Crystal Structure determination by Neutron diffraction}

Crystal structure and the actual Cr concentrations were determined by neutron diffraction. Neutron powder diffraction measurements were performed using BT1 diffractometer at the NIST Center for Neutron Research (NCNR) located in Gaithersburg, Maryland, with the neutron wavelength of $\lambda=1.54 \AA$ at room temperature for $\operatorname{SCGO}(p)$ samples with $p=$ 0.968(6), 0.960(9), 0.917(9), 0.844(8), 0.777(6), 0.736(6), 0.620(8), 0.459(5) and 0.228(5) and $\mathrm{BCGO}(p)$ with $p=0.971(17), 0.943(18), 0.935(9), 0.902(8), 0.806(6), 0.712(6)$, $0.635(7), 0.526(7)$ and $0.417(7)$. The crystal structure and stoichiometry, including the actual $\mathrm{Cr}$ concentrations, were determined by performing a Rietveld refinement using General Structure Analysis System (GSAS) [184].

\section{A.4 Rietveld Refinements of $\operatorname{SCGO}(p)$}

Figure (App.) A.2 shows the neutron powder diffraction data of SCGO $(p=0.968(6)$, $0.844(8), 0.620(8)$ and $0.495(5))$. The lines are the Rietveld refinement results of nuclear structure performed using General Structure Analysis System (GSAS) [184]. The optimal parameters for their crystal structures are listed in Tables S1 to S4, which are consistent with the previously published structure [35]. 


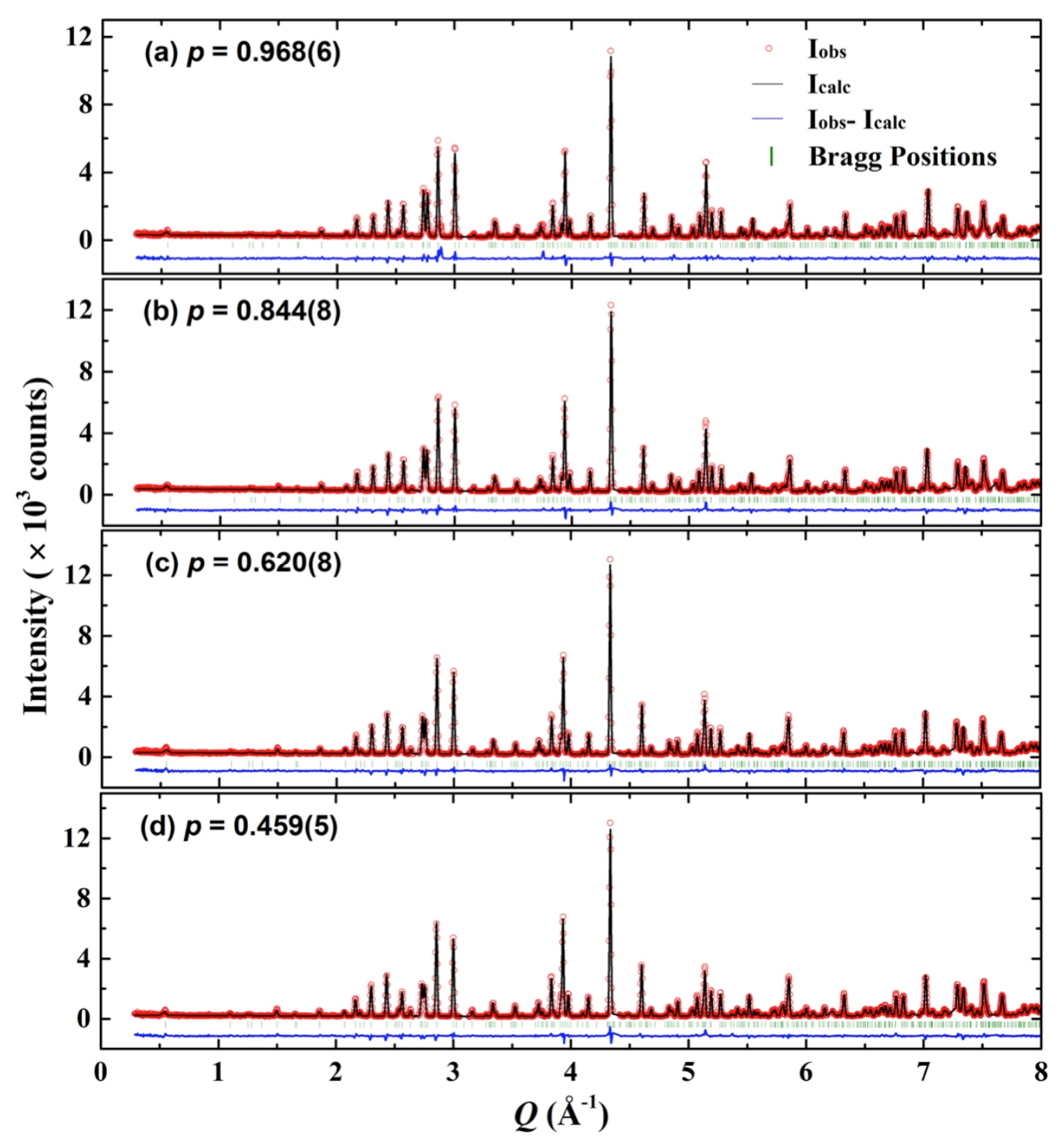

Figure (App.) A.2. Rietveld refinements of $\operatorname{SCGO}(p)$

Neutron powder diffraction data (open circles) and Rietveld refinement patterns (solid curves) for $\operatorname{SCGO}(p)$ samples: (a) $p=0.968(6)$, (b) $p=0.844(8)$, (c) $p=0.620(8)$ and (d) $p=0.459(5)$. The green vertical marks represent the position of Bragg peaks, and the blue solid line at the bottom corresponds to the difference between the observed and calculated intensities. The $p$ values shown in each panel were obtained from the Rietveld refinement.

The Following tables show the Crystal Structure Information of the Rietveld refinements shown in the Figure (App.) A.2. 
Table (App.) A-1 Rietveld analysis of SCGO $p=0.968(6)$.

Positions within space group $P 6_{3} / m m c$ and occupancies per formula unit (f.u.) of atoms in $\operatorname{SCGO}(p)$ at $T=298 \mathrm{~K}$ as determined by Rietveld analysis of the data shown in Fig. S2 using the GSAS. The lattice parameters are $a=5.7948(3) \AA$ and $c=22.6625(2) \AA$. Isotropic Debye-Waller factors, $\exp \left(-\left\langle\boldsymbol{u}^{2}\right\rangle \boldsymbol{Q}^{2}\right)$, were used where $\left\langle\boldsymbol{u}^{2}\right\rangle$ is the mean squared displacement. The resulting overall reduced $\chi^{2}=\mathbf{2 . 5 7 8}$

\begin{tabular}{llllll}
\hline \hline Site & $\boldsymbol{x}$ & $\boldsymbol{y}$ & $\boldsymbol{Z}$ & $\boldsymbol{n} /$ f.u. & $\sqrt{\left\langle\boldsymbol{u}^{2}\right\rangle / \AA}$ \\
\hline $\mathrm{Sr} 2 d$ & $2 / 3$ & $1 / 3$ & $1 / 4$ & 1 & $0.0086(8)$ \\
$\mathrm{Ga} 2 a$ & 0 & 0 & 0 & $0.036(18)$ & $0.0041(4)$ \\
$\mathrm{Cr} 2 a$ & 0 & 0 & 0 & $0.964(18)$ & $0.0041(4)$ \\
$\mathrm{Ga} 2 b$ & 0 & 0 & $1 / 4$ & 1 & $0.0128(7)$ \\
$\mathrm{Ga} 4 f_{\text {iv }}$ & $1 / 3$ & $2 / 3$ & $0.0275(1)$ & 2 & $0.0062(4)$ \\
$\mathrm{Ga} 4 f_{\mathrm{vi}}$ & $1 / 3$ & $2 / 3$ & $0.1911(2)$ & $0.056(30)$ & $0.0041(4)$ \\
$\mathrm{Cr} 4 f_{\mathrm{vi}}$ & $1 / 3$ & $2 / 3$ & $0.1911(2)$ & $1.944(30)$ & $0.0041(4)$ \\
$\mathrm{Ga} 12 k$ & $0.1685(3)$ & $0.3370(6)$ & $-0.1081(1)$ & $0.192(60)$ & $0.0041(4)$ \\
$\mathrm{Cr} 12 k$ & $0.1685(3)$ & $0.3370(6)$ & $-0.1081(1)$ & $5.808(60)$ & $0.0041(4)$ \\
$\mathrm{O} 4 e$ & 0 & 0 & $0.1520(2)$ & 2 & $0.0080(7)$ \\
$\mathrm{O} 4 f$ & $1 / 3$ & $2 / 3$ & $-0.0546(2)$ & 2 & $0.0019(5)$ \\
$\mathrm{O} 6 h$ & $0.1813(3)$ & $0.3635(6)$ & $1 / 4$ & 3 & $0.0086(4)$ \\
$\mathrm{O} 12 k$ & $0.1556(2)$ & $0.3112(4)$ & $0.0529(1)$ & 6 & $0.0037(3)$ \\
$\mathrm{O} 12 k$ & $0.5055(2)$ & $0.0111(4)$ & $0.1507(1)$ & 6 & $0.0059(3)$ \\
\hline \hline
\end{tabular}

Table (App.) A-2 Rietveld analysis of SCGO $p=0.844(8)$.

The lattice parameters at $298 \mathrm{~K}$ are $a=5.79447(3) \AA$ and $c=22.7120$ (2) $\AA$. The resulting overall reduced $\chi^{2}=2.290$.

\begin{tabular}{llllll}
\hline \hline Site & $\boldsymbol{x}$ & $\boldsymbol{y}$ & $\boldsymbol{z}$ & $\boldsymbol{n} /$ f.u. & $\sqrt{\left\langle\boldsymbol{u}^{\mathbf{2}}\right\rangle / \AA}$ \\
\hline $\mathrm{Sr} 2 d$ & $2 / 3$ & $1 / 3$ & $1 / 4$ & 1 & $0.0098(7)$ \\
$\mathrm{Ga} 2 a$ & 0 & 0 & 0 & $0.131(17)$ & $0.0045(3)$ \\
$\mathrm{Cr} 2 a$ & 0 & 0 & 0 & $0.869(17)$ & $0.0045(3)$ \\
$\mathrm{Ga} 2 b$ & 0 & 0 & $1 / 4$ & 1 & $0.0141(7)$ \\
$\mathrm{Ga} 4 f_{\text {iv }}$ & $1 / 3$ & $2 / 3$ & $0.0272(1)$ & 2 & $0.0054(4)$ \\
$\mathrm{Ga} 4 f_{\text {vi }}$ & $1 / 3$ & $2 / 3$ & $0.1906(2)$ & $0.321(28)$ & $0.0044(4)$ \\
$\mathrm{Cr} 4 f_{\text {vi }}$ & $1 / 3$ & $2 / 3$ & $0.1906(2)$ & $1.679(28)$ & $0.0044(4)$ \\
$\mathrm{Ga} 12 k$ & $0.1686(3)$ & $0.3374(5)$ & $-0.1089(1)$ & $0.948(54)$ & $0.0045(4)$ \\
$\mathrm{Cr} 12 k$ & $0.1686(3)$ & $0.3374(7)$ & $-0.1089(1)$ & $5.052(54)$ & $0.0045(4)$ \\
\hline
\end{tabular}




\begin{tabular}{llllll}
\hline $\mathrm{O} 4 e$ & 0 & 0 & $0.1514(1)$ & 2 & $0.0096(6)$ \\
$\mathrm{O} 4 f$ & $1 / 3$ & $2 / 3$ & $-0.0551(1)$ & 2 & $0.0019(5)$ \\
$\mathrm{O} 6 h$ & $0.1809(2)$ & $0.3618(5)$ & $1 / 4$ & 3 & $0.0094(5)$ \\
$\mathrm{O} 12 k$ & $0.1555(2)$ & $0.3109(3)$ & $0.0525(1)$ & 6 & $0.0043(3)$ \\
$\mathrm{O} 12 k$ & $0.5054(2)$ & $0.0108(4)$ & $0.1504(1)$ & 6 & $0.0049(3)$ \\
\hline \hline
\end{tabular}

Table (App.) A-3 Rietveld analysis of SCGO $\boldsymbol{p}=\mathbf{0 . 6 2 0 ( 8 )}$.

The lattice parameters at $298 \mathrm{~K}$ are $a=5.79676(3) \AA$ and $c=22.7541(2) \AA$. The resulting overall reduced $\chi^{2}=2.195$.

\begin{tabular}{llllll}
\hline \hline Site & $\boldsymbol{x}$ & $\boldsymbol{y}$ & $\boldsymbol{Z}$ & $\boldsymbol{n} /$ f.u. & $\sqrt{\left\langle\boldsymbol{u}^{2}\right\rangle / \AA}$ \\
\hline $\mathrm{Sr} 2 d$ & $2 / 3$ & $1 / 3$ & $1 / 4$ & 1 & $0.0107(7)$ \\
$\mathrm{Ga} 2 a$ & 0 & 0 & 0 & $0.298(17)$ & $0.0047(3)$ \\
$\mathrm{Cr} 2 a$ & 0 & 0 & 0 & $0.702(17)$ & $0.0047(3)$ \\
$\mathrm{Ga} 2 b$ & 0 & 0 & $1 / 4$ & 1 & $0.0159(7)$ \\
$\mathrm{Ga} 4 f_{\text {iv }}$ & $1 / 3$ & $2 / 3$ & $0.0276(1)$ & 2 & $0.0044(4)$ \\
$\mathrm{Ga} 4 f_{\mathrm{vi}}$ & $1 / 3$ & $2 / 3$ & $0.1905(1)$ & $0.790(28)$ & $0.0047(3)$ \\
$\mathrm{Cr} 4 f_{\mathrm{vi}}$ & $1 / 3$ & $2 / 3$ & $0.1905(1)$ & $1.210(28)$ & $0.0047(3)$ \\
$\mathrm{Ga} 12 k$ & $0.1682(2)$ & $0.3366(4)$ & $-0.1093(1)$ & $2.328(66)$ & $0.0047(3)$ \\
$\mathrm{Cr} 12 k$ & $0.1682(2)$ & $0.3366(4)$ & $-0.1093(1)$ & $3.672(66)$ & $0.0047(3)$ \\
$\mathrm{O} 4 e$ & 0 & 0 & $0.1504(1)$ & 2 & $0.0102(6)$ \\
$\mathrm{O} 4 f$ & $1 / 3$ & $2 / 3$ & $-0.0547(1)$ & 2 & $0.0019(5)$ \\
O $6 h$ & $0.1811(3)$ & $0.3622(5)$ & $1 / 4$ & 3 & $0.0089(5)$ \\
O $12 k$ & $0.1556(2)$ & $0.3112(3)$ & $0.0526(1)$ & 6 & $0.0046(3)$ \\
$\mathrm{O} 12 k$ & $0.5047(2)$ & $0.0094(4)$ & $0.1502(1)$ & 6 & $0.0051(3)$ \\
\hline \hline
\end{tabular}

Table (App.) A-4 Rietveld analysis of SCGO $p=0.459(5)$.

The lattice parameters at $298 \mathrm{~K}$ are $a=5.79566(3) \AA$ and $c=22.7778(2) \AA$. The resulting overall reduced $\chi^{2}=2.448$.

\begin{tabular}{llllll}
\hline \hline Site & $\boldsymbol{x}$ & $\boldsymbol{y}$ & $\boldsymbol{z}$ & $\boldsymbol{n} /$ f.u. & $\sqrt{\left\langle\boldsymbol{u}^{2}\right\rangle / \AA}$ \\
\hline Sr $2 d$ & $2 / 3$ & $1 / 3$ & $1 / 4$ & 1 & $0.0108(8)$ \\
$\mathrm{Ga} 2 a$ & 0 & 0 & 0 & $0.431(16)$ & $0.0036(3)$ \\
$\mathrm{Cr} 2 a$ & 0 & 0 & 0 & $0.569(16)$ & $0.0036(3)$ \\
$\mathrm{Ga} 2 b$ & 0 & 0 & $1 / 4$ & 1 & $0.0159(7)$ \\
\hline
\end{tabular}




\begin{tabular}{llllll}
\hline Ga $4 f_{\text {iv }}$ & $1 / 3$ & $2 / 3$ & $0.0276(1)$ & 2 & $0.0050(4)$ \\
$\mathrm{Ga} 4 f_{\mathrm{vi}}$ & $1 / 3$ & $2 / 3$ & $0.1897(1)$ & $1.134(13)$ & $0.0037(3)$ \\
$\mathrm{Cr} 4 f_{\mathrm{vi}}$ & $1 / 3$ & $2 / 3$ & $0.1897(1)$ & $0.866(13)$ & $0.0037(3)$ \\
$\mathrm{Ga} 12 k$ & $0.1683(2)$ & $0.3366(4)$ & $-0.1094(1)$ & $3.306(30)$ & $0.0037(3)$ \\
$\mathrm{Cr} 12 k$ & $0.1683(2)$ & $0.3366(4)$ & $-0.1094(1)$ & $2.694(30)$ & $0.0037(3)$ \\
$\mathrm{O} 4 e$ & 0 & 0 & $0.1501(1)$ & 2 & $0.0097(6)$ \\
$\mathrm{O} 4 f$ & $1 / 3$ & $2 / 3$ & $-0.0545(1)$ & 2 & $0.0024(5)$ \\
$\mathrm{O} 6 h$ & $0.1812(3)$ & $0.3623(5)$ & $1 / 4$ & 3 & $0.0093(5)$ \\
$\mathrm{O} 12 k$ & $0.1554(2)$ & $0.3106(3)$ & $0.0526(7)$ & 6 & $0.0054(3)$ \\
$\mathrm{O} 12 k$ & $0.5047(2)$ & $0.0094(4)$ & $0.1500(1)$ & 6 & $0.0061(3)$ \\
\hline \hline
\end{tabular}

\section{A.5 Rietveld Refinements of $\operatorname{BCGO}(p)$}

Figure (App.) A.3 shows the neutron powder diffraction patterns obtained from the $\operatorname{BCGO}(p=0.902(8)), \operatorname{BCGO}(p=0.804(8)), \operatorname{BCGO}(p=0.635(7))$ and $\operatorname{BCGO}(\mathrm{p}=0.417(7))$ samples at room temperature. Rietveld refinements were performed to obtain the crystal structural parameters summarized in Table (App.) A-5, Table (App.) A-6, Table (App.) A-7 and Table (App.) A-8. All of the diffraction peaks could be indexed using the space group $P 63 / m m c$, as for $\operatorname{SCGO}(p)$. The overall occupancies of $\mathrm{Cr} 3+$ ions for these two samples were determined to be 0.902(8), 0.804(8), 0.635(7) and 0.417(7), respectively. 


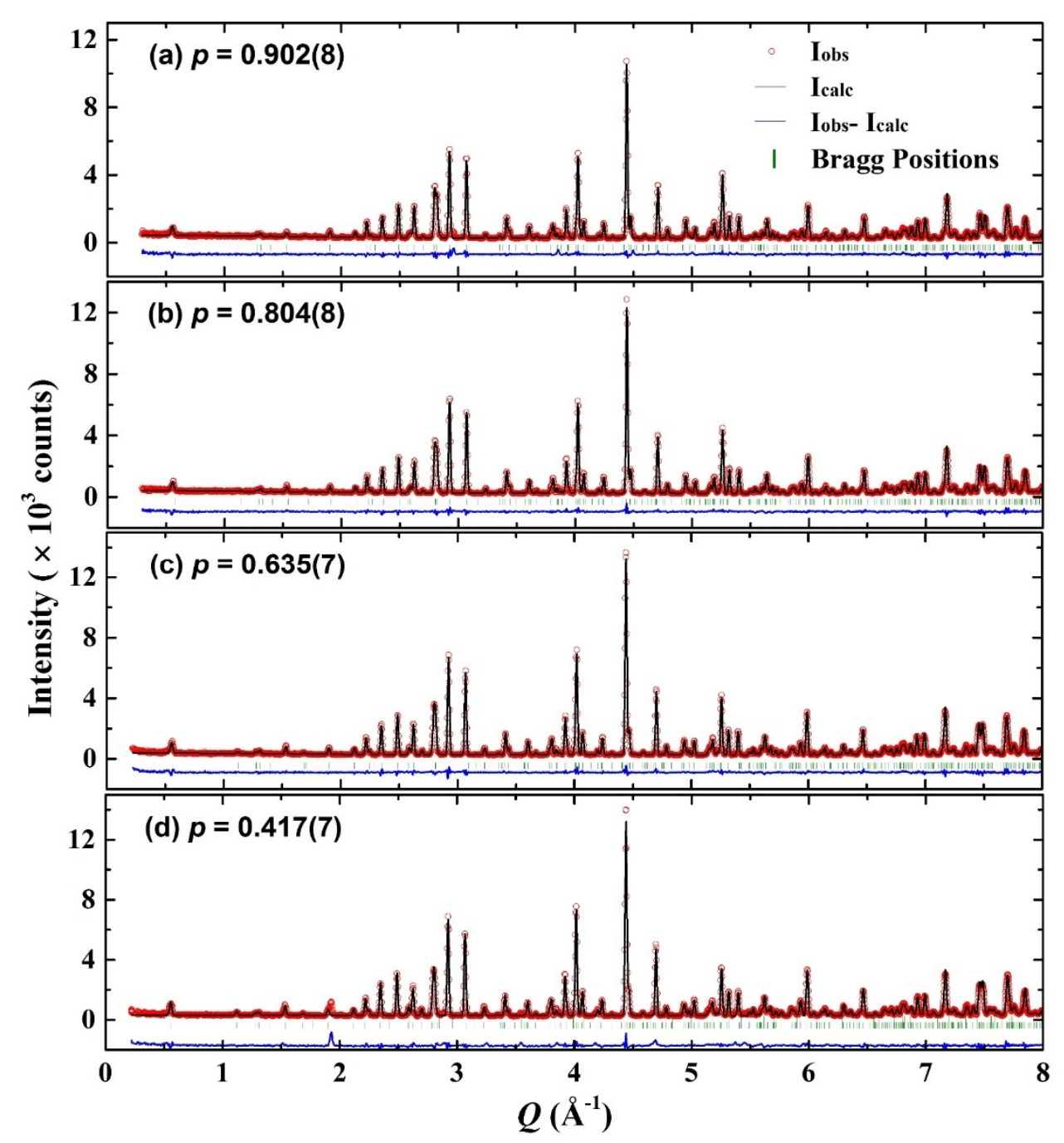

Figure (App.) A.3. Rietveld refinements of $\operatorname{BCGO}(p)$

Neutron powder diffraction data (open circles) and Rietveld refinement patterns (solid curves) for $\operatorname{BCGO}(p)$ samples: (a) $p=0.902(8)$, (b) $p=0.806(6)$, (c) $p=0.621$ (9) and (d) $p=0.417$ (7). The green vertical marks represent the position of Bragg peaks, and the blue solid line at the bottom corresponds to the difference between the observed and calculated intensities. The $p$ values shown in each panel were obtained from the Rietveld refinement.

The Following tables show the Crystal Structure Information of the Rietveld refinements shown in the Figure (App.) A.3. 
Table (App.) A-5 Rietveld analysis of BCGO $p=0.902(8)$.

Positions within space group $\mathrm{P}_{3} / \mathrm{mmc}$ and occupancies per formula unit (f.u.) of atoms in $\mathrm{BCGO}(p)$ at $T=298 \mathrm{~K}$ as determined by Rietveld analysis of the data shown in Fig. S2 using the GSAS. The lattice parameters are $a=5.80374(11) \AA$ and $c=22.8484(5) \AA$. Isotropic Debye-Waller factors, $\exp \left(-\left\langle\boldsymbol{u}^{2}\right\rangle \boldsymbol{Q}^{2}\right)$, were used where $\left\langle\boldsymbol{u}^{2}\right\rangle$ is the mean squared displacement. The resulting overall reduced $\chi^{2}=2.620$

\begin{tabular}{llllll}
\hline \hline Site & $\boldsymbol{x}$ & $\boldsymbol{y}$ & $\boldsymbol{z}$ & $\boldsymbol{n} /$ f.u. & $\sqrt{\left\langle\boldsymbol{u}^{2}\right\rangle / \AA}$ \\
\hline $\mathrm{Ba} 2 d$ & $2 / 3$ & $1 / 3$ & $1 / 4$ & 1 & $0.0012(9)$ \\
$\mathrm{Ga} 2 a$ & 0 & 0 & 0 & $0.075(18)$ & $0.0023(4)$ \\
$\mathrm{Cr} 2 a$ & 0 & 0 & 0 & $0.925(18)$ & $0.0023(4)$ \\
$\mathrm{Ga} 2 b$ & 0 & 0 & $1 / 4$ & 1 & $0.0183(8)$ \\
$\mathrm{Ga} 4 f_{\mathrm{iv}}$ & $1 / 3$ & $2 / 3$ & $0.02758(11)$ & 2 & $0.0054(4)$ \\
$\mathrm{Ga} 4 f_{\mathrm{vi}}$ & $1 / 3$ & $2 / 3$ & $0.19032(17)$ & $0.218(30)$ & $0.0023(4)$ \\
$\mathrm{Cr} 4 f_{\mathrm{vi}}$ & $1 / 3$ & $2 / 3$ & $0.19032(17)$ & $1.782(30)$ & $0.0023(4)$ \\
$\mathrm{Ga} 12 k$ & $0.16805(28)$ & $0.3361(6)$ & $-0.10707(9)$ & $0.588(60)$ & $0.0023(4)$ \\
$\mathrm{Cr} 12 k$ & $0.16805(28)$ & $0.3361(6)$ & $-0.10707(9)$ & $5.412(60)$ & $0.0023(4)$ \\
$\mathrm{O} 4 e$ & 0 & 0 & $0.15020(13)$ & 2 & $0.0077(7)$ \\
O $4 f$ & $1 / 3$ & $2 / 3$ & $-0.05479(15)$ & 2 & $0.0021(5)$ \\
$\mathrm{O} 6 h$ & $0.18218(28)$ & $0.3643(6)$ & $1 / 4$ & 3 & $0.0063(5)$ \\
$\mathrm{O} 12 k$ & $0.15617(19)$ & $0.3122(4)$ & $0.05225(7)$ & 6 & $0.00598(32)$ \\
$\mathrm{O} 12 k$ & $0.50246(22)$ & $0.0049(4)$ & $0.14871(6)$ & 6 & $0.00470(27)$ \\
\hline \hline
\end{tabular}

Table (App.) A-6 Rietveld analysis of BCGO $p=0.804(8)$.

The lattice parameters at $298 \mathrm{~K}$ are $a=5.80573(8) \AA$ and $c=22.8727(4) \AA$. The resulting overall reduced $\chi^{2}=\mathbf{2 . 1 0 5}$.

\begin{tabular}{lllllc}
\hline \hline Site & $\boldsymbol{x}$ & $\boldsymbol{y}$ & $\boldsymbol{z}$ & $\boldsymbol{n} /$ f.u. & $\sqrt{\left\langle\boldsymbol{u}^{2}\right\rangle / \AA}$ \\
\hline $\mathrm{Ba} 2 d$ & $2 / 3$ & $1 / 3$ & $1 / 4$ & 1 & $0.0014(8)$ \\
$\mathrm{Ga} 2 a$ & 0 & 0 & 0 & $0.222(12)$ & $0.00247(31)$ \\
$\mathrm{Cr} 2 a$ & 0 & 0 & 0 & $0.778(12)$ & $0.00247(31)$ \\
$\mathrm{Ga} 2 b$ & 0 & 0 & $1 / 4$ & 1 & $0.0193(7)$ \\
$\mathrm{Ga} 4 f_{\text {iv }}$ & $1 / 3$ & $2 / 3$ & $0.02775(9)$ & 2 & $0.0049(4)$ \\
\hline
\end{tabular}




\begin{tabular}{llllll}
\hline $\mathrm{Ga} 4 f_{\mathrm{vi}}$ & $1 / 3$ & $2 / 3$ & $0.18982(13)$ & $0.444(24)$ & $0.00247(31)$ \\
$\mathrm{Cr} 4 f_{\mathrm{vi}}$ & $1 / 3$ & $2 / 3$ & $0.18982(13)$ & $1.556(24)$ & $0.00247(31)$ \\
$\mathrm{Ga} 12 k$ & $0.16764(20)$ & $0.3353(4)$ & $-0.10738(6)$ & $1.176(48)$ & $0.00247(31)$ \\
$\mathrm{Cr} 12 k$ & $0.16764(20)$ & $0.3353(4)$ & $-0.10738(6)$ & $4.824(48)$ & $0.00247(31)$ \\
$\mathrm{O} 4 e$ & 0 & 0 & $0.14981(11)$ & 2 & $0.0067(6)$ \\
$\mathrm{O} 4 f$ & $1 / 3$ & $2 / 3$ & $-0.05457(12)$ & 2 & $0.0014(4)$ \\
$\mathrm{O} 6 h$ & $0.18206(22)$ & $0.3640(4)$ & $1 / 4$ & 3 & $0.0061(4)$ \\
$\mathrm{O} 12 k$ & $0.15570(15)$ & $0.31130(31)$ & $0.05218(6)$ & 6 & $0.00607(26)$ \\
$\mathrm{O} 12 k$ & $0.50275(18)$ & $0.0055(4)$ & $0.14860(5)$ & 6 & $0.00534(23)$ \\
\hline \hline
\end{tabular}

Table (App.) A-7 Rietveld analysis of BCGO $p=0.635(7)$.

The lattice parameters at $298 \mathrm{~K}$ are $a=5.80698(8) \AA$ and $c=22.9074(4) \AA$. The resulting overall reduced $\chi^{2}=2.311$.

\begin{tabular}{llllll}
\hline \hline Site & $\boldsymbol{x}$ & $\boldsymbol{y}$ & $\boldsymbol{Z}$ & $\boldsymbol{n} /$ f.u. & $\sqrt{\left\langle\boldsymbol{u}^{2}\right\rangle / \AA}$ \\
\hline Ba $2 d$ & $2 / 3$ & $1 / 3$ & $1 / 4$ & 1 & $0.0019(8)$ \\
$\mathrm{Ga} 2 a$ & 0 & 0 & 0 & $0.236(16)$ & $0.0026(3)$ \\
$\mathrm{Cr} 2 a$ & 0 & 0 & 0 & $0.764(16)$ & $0.0026(3)$ \\
$\mathrm{Ga} 2 b$ & 0 & 0 & $1 / 4$ & 1 & $0.0218(7)$ \\
$\mathrm{Ga} 4 f_{\text {iv }}$ & $1 / 3$ & $2 / 3$ & $0.02776(9)$ & 2 & $0.0040(4)$ \\
$\mathrm{Ga} 4 f_{\mathrm{vi}}$ & $1 / 3$ & $2 / 3$ & $0.18972(11)$ & $0.776(26)$ & $0.0026(3)$ \\
$\mathrm{Cr} 4 f_{\mathrm{vi}}$ & $1 / 3$ & $2 / 3$ & $0.18972(11)$ & $1.224(28)$ & $0.0026(3)$ \\
$\mathrm{Ga} 12 k$ & $0.16767(17)$ & $0.33534(34)$ & $-0.10802(6)$ & $2.274(54)$ & $0.0026(3)$ \\
$\mathrm{Cr} 12 k$ & $0.16767(17)$ & $0.33534(34)$ & $-0.10802(6)$ & $3.726(54)$ & $0.0026(3)$ \\
O $4 e$ & 0 & 0 & $0.14936(11)$ & 2 & $0.0073(6)$ \\
O $4 f$ & $1 / 3$ & $2 / 3$ & $-0.05416(12)$ & 2 & $0.0019(4)$ \\
O $6 h$ & $0.18248(23)$ & $0.3649(5)$ & $1 / 4$ & 3 & $0.0069(4)$ \\
O $12 k$ & $0.15567(16)$ & $0.31124(31)$ & $0.05219(6)$ & 6 & $0.0063(3)$ \\
O $12 k$ & $0.50321(19)$ & $0.0064(4)$ & $0.14842(5)$ & 6 & $0.0054(3)$ \\
\hline \hline
\end{tabular}

Table (App.) A-8 Rietveld analysis of BCGO $p=0.417$ (7).

The lattice parameters at $298 \mathrm{~K}$ are $a=5.8066(1) \AA$ and $c=22.9319(5) \AA$. The resulting overall reduced $\chi^{2}=\mathbf{4 . 9 3 5}$.

\begin{tabular}{llllll}
\hline \hline Site & $\boldsymbol{x}$ & $\boldsymbol{y}$ & $\boldsymbol{z}$ & $\boldsymbol{n} /$ f.u. & $\sqrt{\left\langle\boldsymbol{u}^{2}\right\rangle / \AA}$ \\
\hline Ba $2 d$ & $2 / 3$ & $1 / 3$ & $1 / 4$ & 1 & $0.00046(8)$ \\
$\mathrm{Ga} 2 a$ & 0 & 0 & 0 & $0.482(22)$ & $0.00422(3)$ \\
$\mathrm{Cr} 2 a$ & 0 & 0 & 0 & $0.518(22)$ & $0.00422(3)$ \\
Ga $2 b$ & 0 & 0 & $1 / 4$ & 1 & $0.02403(7)$ \\
\hline
\end{tabular}




\begin{tabular}{llllll}
\hline Ga $4 f_{\text {iv }}$ & $1 / 3$ & $2 / 3$ & $0.02781(13)$ & 2 & $0.00419(4)$ \\
Ga $4 f_{\text {vi }}$ & $1 / 3$ & $2 / 3$ & $0.18885(15)$ & $1.114(34)$ & $0.00422(3)$ \\
Cr $4 f_{\text {vi }}$ & $1 / 3$ & $2 / 3$ & $0.18885(15)$ & $0.886(34)$ & $0.00422(3)$ \\
Ga $12 k$ & $0.16803(24)$ & $0.3361(5)$ & $-0.10830(7)$ & $3.648(48)$ & $0.00422(3)$ \\
Cr $12 k$ & $0.16803(24)$ & $0.3361(5)$ & $-0.10830(7)$ & $2.352(48)$ & $0.00422(3)$ \\
O $4 e$ & 0 & 0 & $0.14884(16)$ & 2 & $0.00695(6)$ \\
O $4 f$ & $1 / 3$ & $2 / 3$ & $-0.05398(18)$ & 2 & $0.00035(5)$ \\
O $6 h$ & $0.18303(31)$ & $0.3660(6)$ & $1 / 4$ & 3 & $0.0083(5)$ \\
O $12 k$ & $0.15552(21)$ & $0.3109(4)$ & $0.05211(9)$ & 6 & $0.0065(3)$ \\
O $12 k$ & $0.50308(30)$ & $0.0061(6)$ & $0.14836(7)$ & 6 & $0.00539(3)$ \\
\hline \hline
\end{tabular}




\section{A.6 Comparison of SCGO(p), BCGO(p) and BSZGCO}

Table (App.) A-9 Summery of $T_{\mathrm{DC}}$ and $\theta_{c w}$ for $\mathrm{BCGO}(p), \operatorname{SCGO}(p)$ and BSZGCO.

The transition temperature $T_{D C}$ is estimated as the cusp temperature of the ZFC susceptibility as shown in the Figure 4.3 and Figure (App.) A.1 (b). The Curie-Weiss temperature $\theta_{c w}$ is determined by the fittings to the reciprocal high temperature susceptibility as shown in the Figure 4.2 and Figure (App.) A.1 (a). The frustration index $f$ of each sample is defined as $f=\left|\theta_{c w}\right| / T_{D C}$.

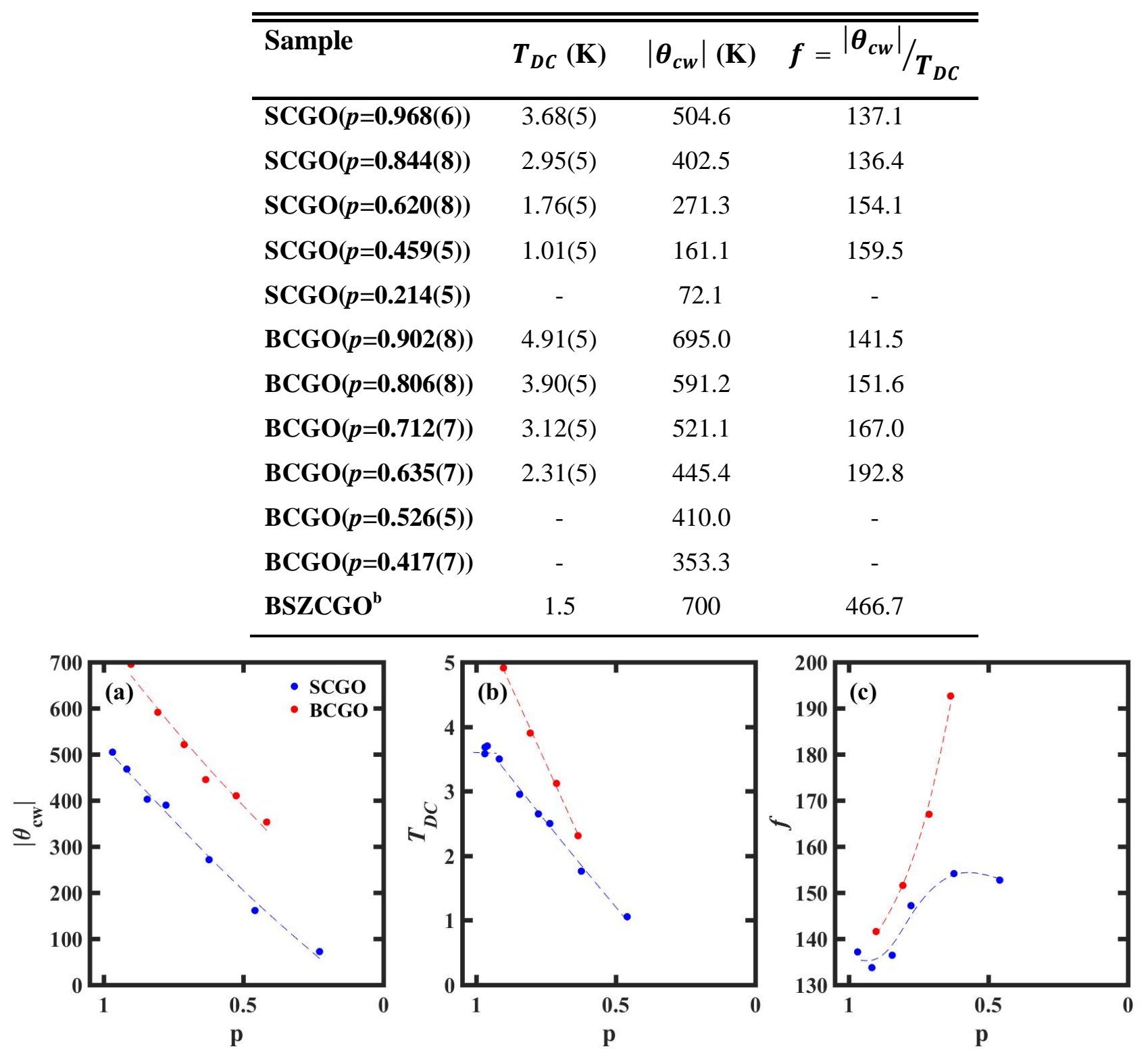

Figure (App.) A.4. Comparison of $\operatorname{SCGO}(p)$ versus BCGO(p)

(a) The Curie-Weiss Temperature $\theta_{c w}$, (b) the freezing temperature $T_{D C}$ and (c) frustration index $f$ of $\operatorname{SCGO}(p)$ and $\mathrm{BCGO}(p)$ samples are shown here as a function of dilution $p$. The dash lines are guides to the eye for each dataset. 


\section{A.7 Time-of-flight Neutron Scattering Measurements}

Time-of-flight neutron scattering measurements were performed using the Disk-ChopperSpectrometer (DCS) at NCNR with $\lambda=6 \AA . \operatorname{SCGO}(p)$ samples with $p=0.968(6)$, $0.960(9), 0.917(9), 0.844(8), 0.777(6), 0.736(6)$ and 0.620(8) were measured in a standard DCS ILL orange cryostat and $p=0.459(5)$ was measured with a Helium-3 insert. The low energy inelastic magnetic neutron scattering spectrum was measured at various temperatures, $T$, around $T_{f}$ for the samples. For each $\operatorname{SCGO}(p)$ sample, an energy dependent background was determined by imposing the detailed balance constraint $S(-\hbar \omega, T)=$ $e^{-\frac{\hbar \omega}{k_{B} T}} S(\hbar \omega, T)$ where $k_{B}$ is the Boltzmann constant, and it was subtracted from the data to obtain the magnetic contribution. Then, the imaginary part of the dynamic susceptibility was derived from the fluctuation dissipation theorem, $\chi^{\prime \prime}(\hbar \omega)=\pi\left\{1-e^{-\frac{\hbar \omega}{k_{B} T}}\right\} S(\hbar \omega)$.

\section{A.8 Ultra-Low Field Measurements and Thermoremanent Magnetization}

The Thermoremanent Magnetization (TRM) data, shown in Figure 6.2, Figure 6.3, Figure 7.1, Figure 7.3 and Figure 7.4, were collected using the following procedure. First, each sample was cooled down from well above the freezing temperature, $T_{f}$, to base temperature with a single stop for a period of time, $t_{w}$, at an intermediate temperature $T_{w}$ below $T_{f}$ under zero field. Once cooled down to base temperature, the thermoremanent magnetization is measured by applying a small field of a few gauss upon heating at a constant rate. For all the measurements reported in this paper, we used a Superconducting Quantum Inference Device (SQUID) magnetometer, Quantum Design MPMS-XL5 equipped with the ultra-low-field option together with the environmental magnetic shield. Since it is necessary to have zero-field conditions at the sample during the cooling process including the waiting at an intermediate temperature, the remnant magnetic field at the sample position was measured by the instrument's fluxgate, and has been eliminated by introducing a compensating field using non-superconducting DC coil to get the remaining uncompensated magnetic field less than $0.005 \mathrm{G}$ at the sample position. After that, a small DC magnetic field of $3 \mathrm{G}$ was generated by the DC non-superconducting coil and applied to the sample during the TRM measurements. 


\section{A.9 $\chi$ and inelastic order parameter as a function of relaxation time}

The Following figure summarizes,
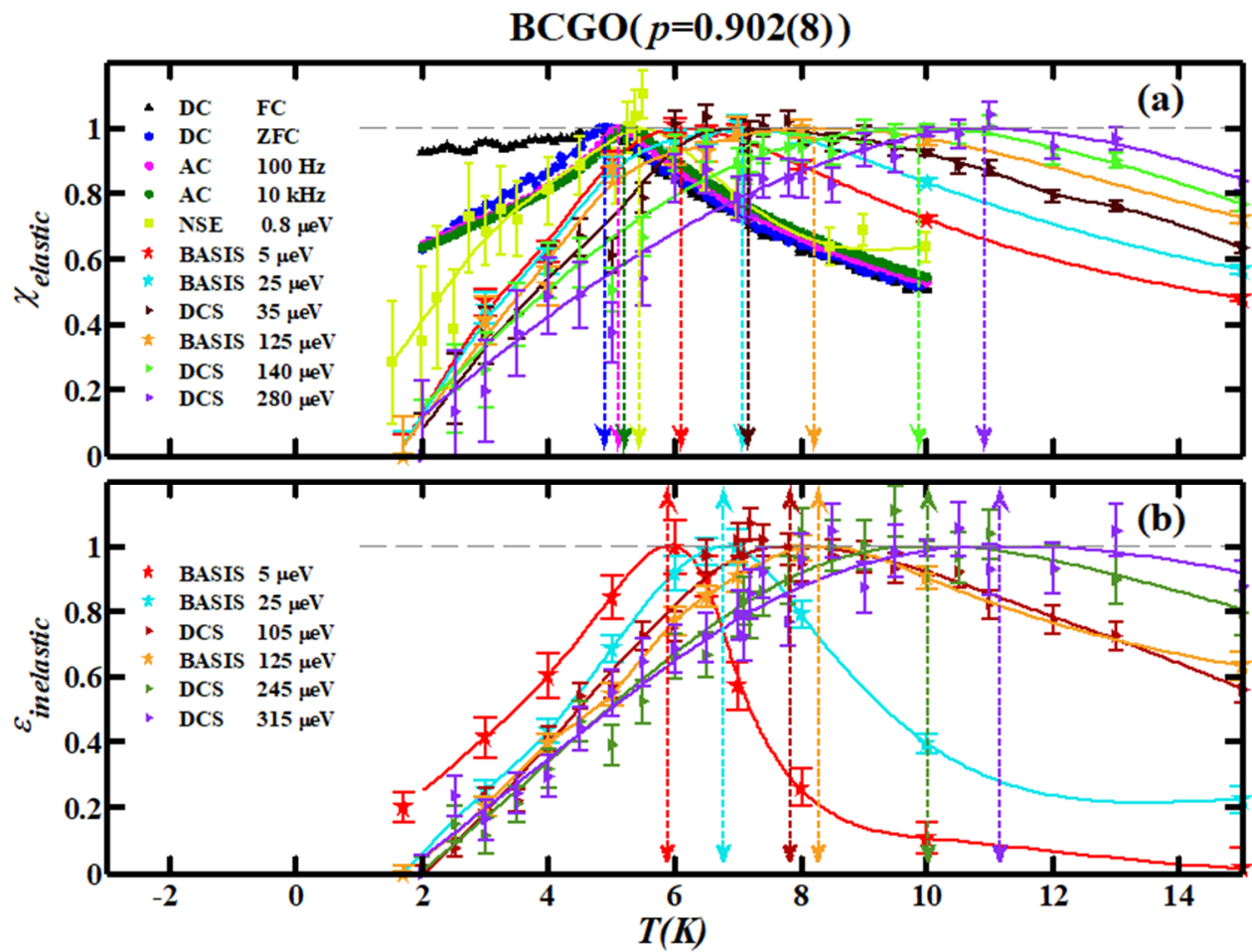

Figure (App.) A.5. Susceptibility and inelastic order parameter for $\operatorname{BCGO}(p=0.902(8))$

(a) shows the DC susceptibility $\left(\chi_{D C}\right)$ and AC susceptibility $\left(\chi_{A C}\right)$ and susceptibility calculated using elastic order-parameters $\left(I\left(Q_{0}, \Delta \omega_{0}, T\right)\right)$ measured from neutron scattering experiments at different energy resolutions $\left(\Delta \omega_{0}\right)$ for $\operatorname{BCGO}(p=0.902(8))$. The Equation 5.3 was used to calculate the susceptibility from $I\left(Q_{0}, \Delta \omega_{0}, T\right)$ data. (b) shows the inelastic order-parameter ( $\left.\varepsilon_{\text {inelastic }}\right)$ as a function of energy resolution $\left(\Delta \omega_{0}\right)$. The Neutron Spin echo (NSE) spectrometer at NCNR, NIST, Neutron Backscattering spectrometer (BASIS) at SNS, ORNL and Disk Chopper Spectrometer (DCS) at NCNR, NIST were used to measure $I\left(Q_{0}, \Delta \omega_{0}, T\right)$ as a function of $\Delta \omega_{0}$ and $T$. 


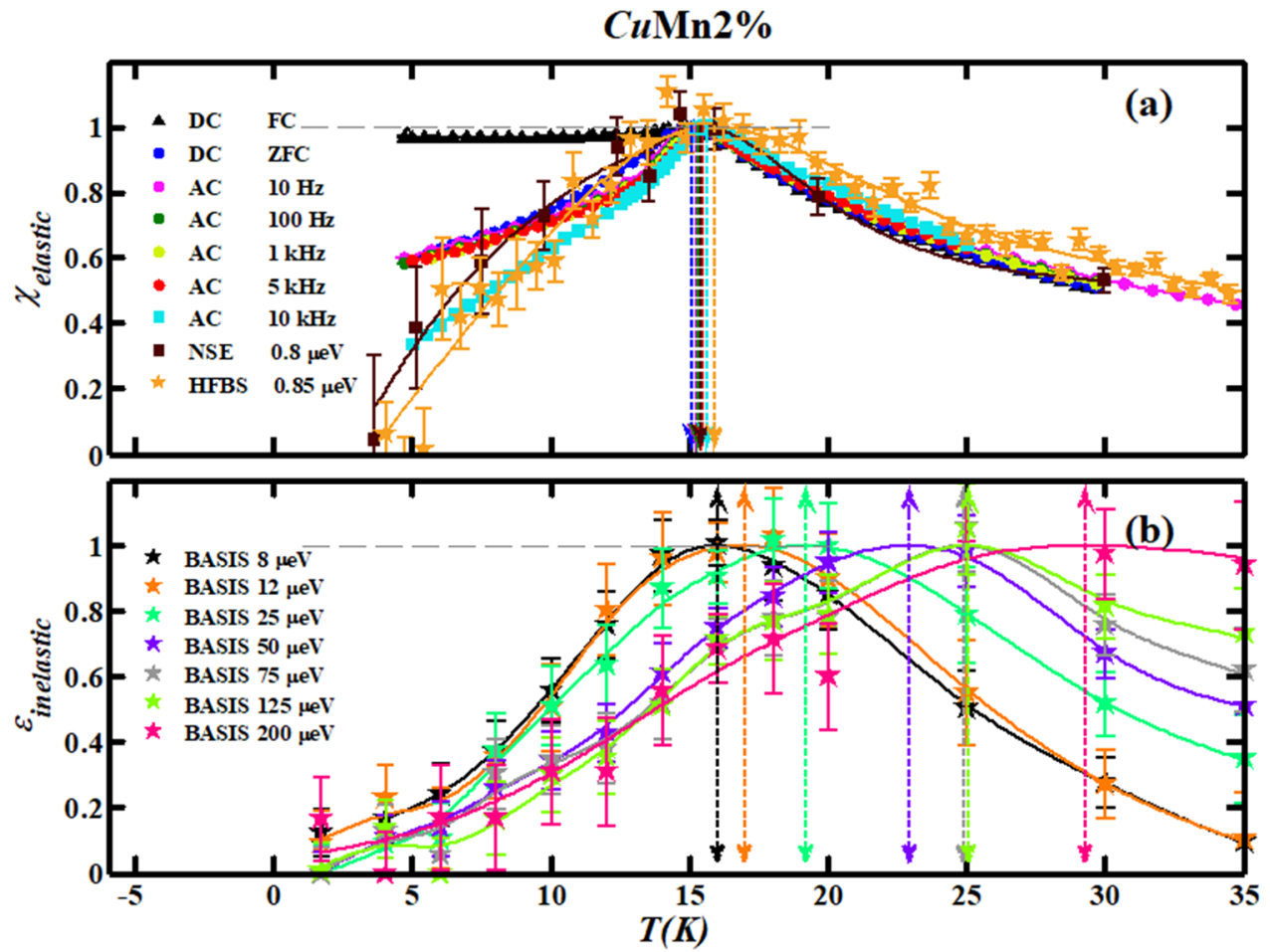

Figure (App.) A.6. Susceptibility and inelastic order parameter for $\mathrm{CuMn2 \%}$

(a) shows the DC susceptibility $\left(\chi_{D C}\right)$ and AC susceptibility $\left(\chi_{A C}\right)$ and susceptibility calculated using elastic order-parameters $\left(I\left(Q_{0}, \Delta \omega_{0}, T\right)\right)$ measured from neutron scattering experiments at different energy resolutions $\left(\Delta \omega_{0}\right)$ for $C u \mathrm{Mn} 2 \%$. The Equation 5.3 was used to calculate the susceptibility from $I\left(Q_{0}, \Delta \omega_{0}, T\right)$ data. (b) shows the inelastic order-parameter $\left(\varepsilon_{\text {inelastic }}\right)$ as a function of energy resolution $\left(\Delta \omega_{0}\right)$. The Neutron Spin echo (NSE) spectrometer at NCNR, NIST, Neutron Backscattering spectrometer (BASIS) at SNS, ORNL and High Flux Backscattering Spectrometer (HFBS) at NCNR, NIST were used to measure $I\left(Q_{0}, \Delta \omega_{0}, T\right)$ as a function of $\Delta \omega_{0}$ and $T$. 

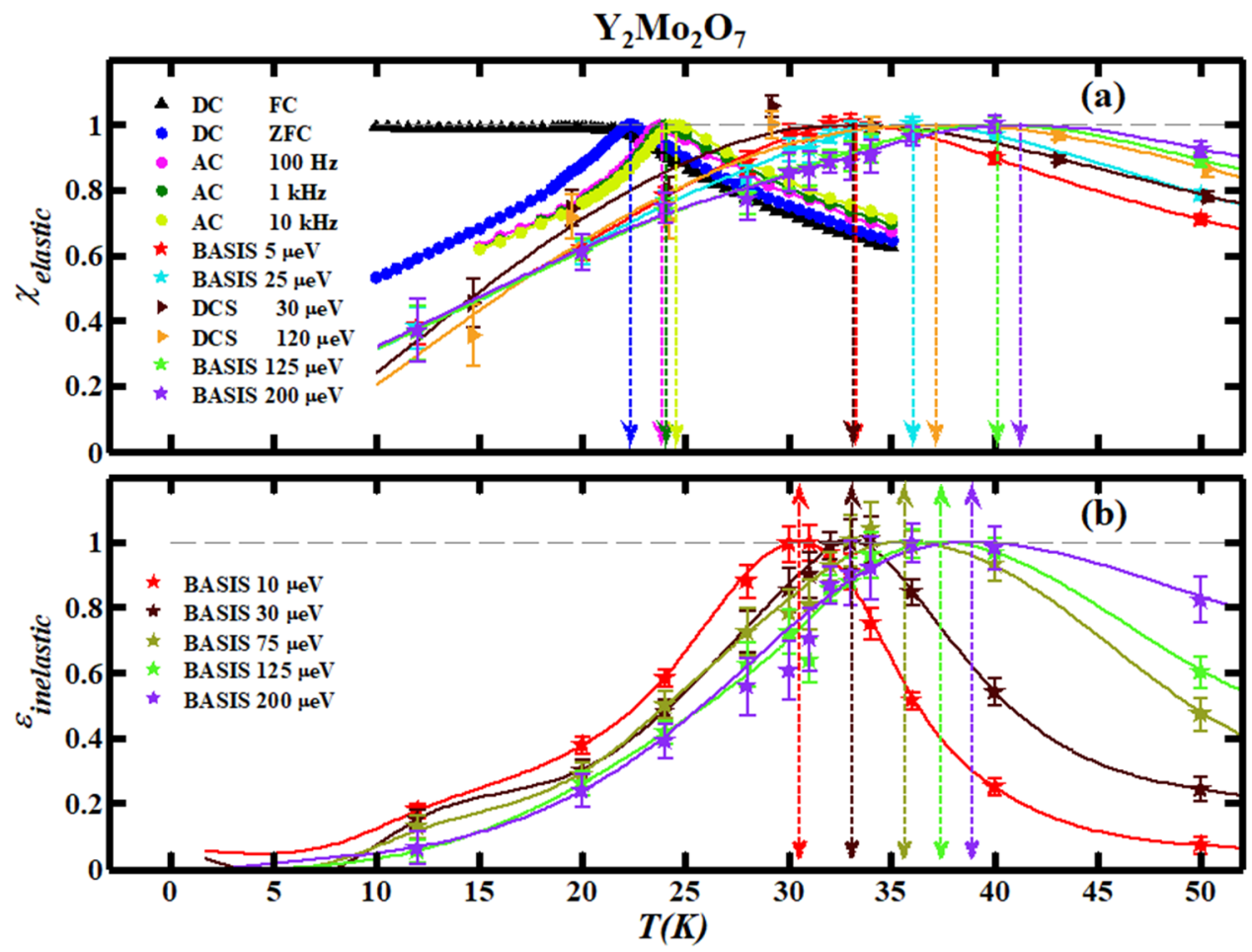

Figure (App.) A.7. Susceptibility and inelastic order parameter for $\mathrm{Y}_{2} \mathrm{Mo}_{2} \mathrm{O}_{7}$

(a) shows the DC susceptibility $\left(\chi_{D C}\right)$ and AC susceptibility $\left(\chi_{A C}\right)$ and susceptibility calculated using elastic order-parameters $\left(I\left(Q_{0}, \Delta \omega_{0}, T\right)\right)$ measured from neutron scattering experiments at different energy resolutions $\left(\Delta \omega_{0}\right)$ for $\mathrm{Y}_{2} \mathrm{Mo}_{2} \mathrm{O}_{7}$. The Equation 5.3 was used to calculate the susceptibility from $I\left(Q_{0}, \Delta \omega_{0}, T\right)$ data. (b) shows the inelastic order-parameter $\left(\varepsilon_{\text {inelastic }}\right)$ as a function of energy resolution $\left(\Delta \omega_{0}\right)$. The Neutron Spin echo (NSE) spectrometer at SNS, ORNL, Neutron Backscattering spectrometer (BASIS) at SNS, ORNL and Disk Chopper Spectrometer (DCS) at NCNR, NIST were used to measure $I\left(Q_{0}, \Delta \omega_{0}, T\right)$ as a function of $\Delta \omega_{0}$ and $T$. 


\section{Appendix B Spinwave Dispersion for Kagome-Triangular- Kagome Trilayer}

Author: Kazuki Iida [72]

The spin wave calculations were done for the SCGO at the clean limit, $p=1$. This was done by expanding around classically ordered ground state configuration to harmonic order, using the standard Holstein-Primakoff method [185].

The chosen states are coplanar spin configurations that are described in the inset in Fig. S6. The spin wave dispersion is plotted along the $(H, H, 1.8)$ momentum direction for various values of $\alpha=\cos ^{-1}\left(\frac{J^{\prime} J+1}{2}\right)(S 5)$, going from $\alpha=0$ for the ideal SCGO to $\alpha=\pi / 3$ for isolated kagome.

The spin wave analysis shows a flat band of 9 zero modes, indicating a large classical degeneracy.
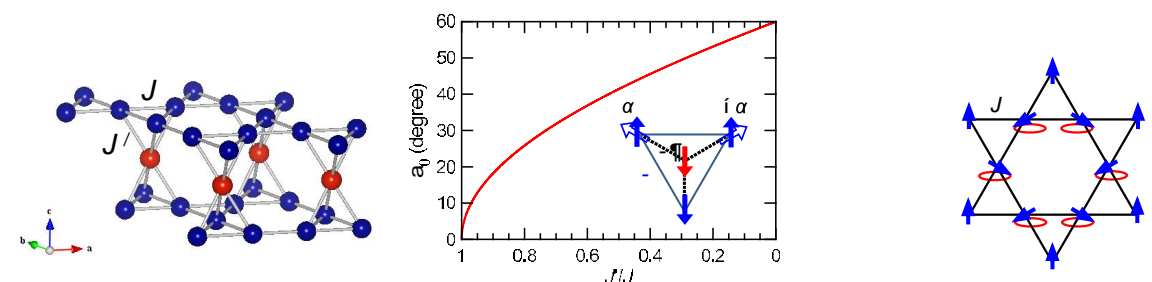

$\overleftarrow{1}$

$J^{\prime} / J$

0
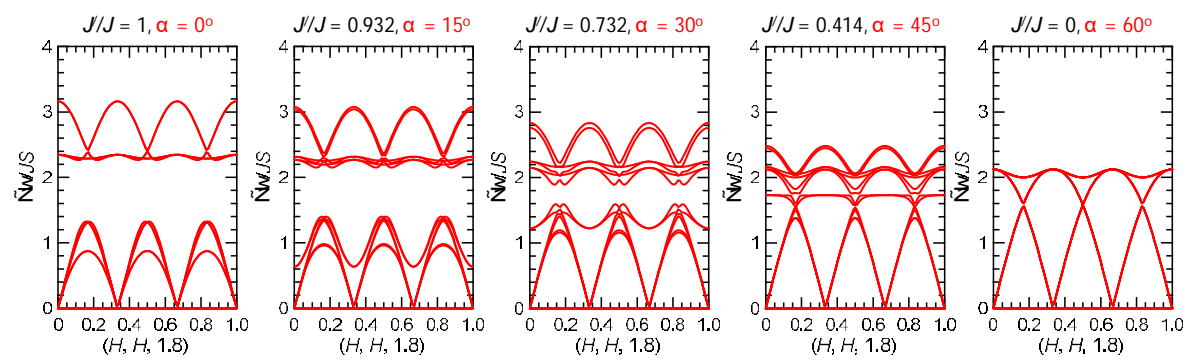

Figure (App.) B.1. Spin wave dispersion.

The ideal SCGO lattice and the kagome may be viewed as limits as $J^{\prime} / J$ goes from 1 to 0 . Ordered classical coplanar ground state configurations may be obtained from the ideal case by collectively rotating a subset of the spins as is shown in the inset and Ref. [15]. 


\section{Appendix C Barrier Tree Models and Monte Carlo Simulations}

Authors: Tianran Chen, Gia-Wei Chern and Seung-Hun Lee [186]

Understanding the dynamics of a glassy system via the energy landscape approach has led to promising insights into puzzling phenomena, such as temperature-dependent aging and memory effect. In this framework, the energy landscape is seen as a set of basins of attraction, and the system evolves through a succession of jumps between their local minima [187,188]. This approach focuses on the interbasin transitions without treating explicitly the fast (high-frequency) intrabasin dynamics. Over the past decades, much effort has been devoted to characterizing the structure and topology of the energy landscape for various glass-forming systems $[83,95,189]$. In particular, the so-called disconnectivity graph [95] has become a widely used approach for visualizing and representing the multidimensional potential energy surface. The disconnectivity graph summarizes the local minima and saddle points of an energy landscape into a tree. Each leaf in this tree representation corresponds to a local minimum, whereas the branching point is a transition state (saddle point) connecting different local minima. Another approach to describe the energy landscape is to use the language of complex networks [100,188].

The disconnectivity graph (also referred to as a barrier tree representation) can be constructed numerically from the database of local minima and the kinetic pathways for small molecules or lattices [189]. Monte Carlo sampling is often required to construct the representative barrier tree for a larger system. These approaches require microscopic details of the system at hand, which are hard to process for complicated physical systems. An alternative, phenomenological, approach for complex systems uses a statistically based characterization of the barrier tree, which is the method adopted in our work. For a given energy landscape representation, either through a barrier tree or through a complex 
network, the dynamics of the system can be simulated as a random walk on the tree or the network. Amaster equation is often used to study the resultant dynamics [95,187,188]. Here we use the Markov chain Monte Carlo simulations coupled with a dynamical tree method to study thememory effect of spin glass and spin jam states.Our Monte Carlo approach offers the advantage of being applicable to barrier trees with complex structures without the need of introducing further approximations as in the master equation method.

In our simulations, the relaxation dynamics of the system are modeled as a random walk on the barrier tree. Each node of the tree, corresponding to either a local minimum or a saddle point, represents a specific microscopic spin configuration. Transition between two nodes corresponds to modifying a small number of spins. In our simulations, the structure and properties of the barrier trees are characterized statistically. Specifically, the tree is described by a set of random numbers satisfying certain probability distributions.

We first discuss the statistical description of the barrier tree for a conventional spin glass. Here, the tree has a hierarchical structure with many levels. A node at a lower level (larger $l$ ) corresponds to a lower energy state (Figure 6.1 (a)). The barrier energy $\varepsilon$ at level $l$ is an independent random variable with an exponential distribution $p(\varepsilon)=e^{-\varepsilon / T_{l}} / T_{l}$ characterized by the temperature $T_{l}$. This construction is similar to the so-called random energy or random trap models $[190,191]$ that are shown to exhibit the characteristic aging behavior in spin glasses.

The characteristic temperature $T_{l}$ decreases with increasing levels; i.e., $T_{1}>T_{2}>\cdots>$ $T_{l_{\max }}$, corresponding to smaller energy barriers at the bottom of the hierarchical tree. In our simulations, we assume that the characteristic temperature $T_{l} \sim T_{1} e^{-\alpha l}$ decreases exponentially with the level index. The relaxation of the system in this hierarchical structure exhibits complex temperature- dependent dynamics. Typically, because the relaxation time at level $l$ scales as $\tau_{l} \sim \tau_{0} e^{\varepsilon_{l} / T}$, where $\tau_{0}$ is a microscopic time scale, the relaxation dynamics start to show exponential slowing down at level $l$ when $T<T_{l}$. Interestingly, the progressive slowing down of the relaxation dynamics can be viewed as the fact that the system undergoes a series of glass transitions with decreasing temperature. The largest energy scale $T_{1}$ then determines the nominal freezing transition temperature $T_{f}$. This hierarchical construction is consistent with the picture of temperature-dependent 
energy barriers [132], which is shown to be crucial for the occurrence of memory effect in conventional spin glass.

At each level, whether the node is a local minimum (and thus a dead end) or a saddle point is specified by a constant $0<\lambda_{l}<1$; i.e., $\lambda_{l}$ is the probability that a given node at level $l$ is a local minimum. Another random number $n_{b}$ is used to specify the branching or the number of descendants of a saddle point. Finally, for the calculation of magnetic susceptibility, we use the simple random magnetization model discussed in ref. [94] for the barrier tree. Specifically, the magnetization of a particular state at level $L$ is given by $M=m_{0}+m_{1}+\cdots+m_{L}$. The magnetization contribution $m_{l}$ from level $l$ is a random number uniformly distributed in the interval $\left[-M_{l}, M_{l}\right]$, where the bound $M_{l}$ is assumed to decrease exponentially with increasing levels. Consequently, a Zeeman coupling $H_{z}=$ $-H \cdot M$ is included in the Monte Carlo simulations of the reheating process.

We use the standard Metropolis dynamics in our Monte Carlo simulations. Because the barrier tree is specified only statistically, there is no need to create a tree at the beginning of the dynamical simulations. Instead, we generate the barrier tree dynamically according to the desired statistical properties as discussed above. However, additional bookkeeping is required to describe a system currently at level $L$. Specifically, we need to keep track only of all of the barrier energies and magnetizations from levels $l \leq L$, i.e., $\left\{\varepsilon_{1}, \varepsilon_{2}, \cdots, \varepsilon_{l}, \cdots, \varepsilon_{L}\right\}$ and $\left\{m_{1}, m_{2}, \cdots, m_{l}, \cdots, m_{L}\right\}$. A Monte Carlo step then consists of the following procedures: $(i)$ Determine whether this node is a local minimum (a dead end) or a saddle point. This can be done by generating a random number $\mathrm{r}$ uniformly distributed between 0 and 1 . If $r<\lambda_{L}$, then the current state is a local minimum, and the system can move only upward to escape this local trap. (ii) If the current node is a saddle point, then we generate another uniformly distributed random number $r^{\prime} \in[0,1]$. If $r^{\prime}>1 /\left(n_{b}+1\right)$, then we attempt to move the system upward. Otherwise we move the system downward to a lower level (closer to the global minimum). (iii) For a downhill update, we first increase the level by one. Next, we generate new random numbers $\varepsilon_{L+1}$ and $m_{L+1}$ according to the respective probability distribution and add them to the lists of barrier energies and magnetizations, respectively. (iv) Finally, for an uphill update we first compute the energy $\operatorname{cost} \Delta \varepsilon=\varepsilon_{L}+H m_{L}$. Then a standard Metropolis criterion is used to determine whether 
this upward movement is accepted or not. If the uphill move is accepted, we then erase $\varepsilon_{L}$ and $m_{L}$ from the respective lists. The above procedures are illustrated in Figure (App.) C.1.

We note that the dynamical tree simulation is valid as long as the number of branching $n_{b} \gg 1$ (in the simulations we took $n_{b} \sim 500$ ). Under this condition, we can neglect the possibility that the system will visit exactly the same lower-energy states more than once in our finite-time simulation. We also find that a rather large $\lambda_{l}$ is required to observe a noticeable memory effect. This condition simply means that there are many dead-end local traps along the way toward the global ground state, which is consistent with the rugged funnel-type energy landscape. In our simulations, we assumed a maximum number of level $l_{\max }=50$ and took $\lambda_{l}$ to increase linearly from 0.9 to 1 at $l_{\max }$. We also note that barrier trees characterized by these statistical properties are similar to the "palm tree" pattern in the classification of disconnectivity graphs $[83,189]$.

The spin jam glassy state, on the other hand, is characterized by a very different energy landscape. This is because the numerous minima in the spin jam originate from quantum fluctuations that lift the otherwise flat energy surface at the classical level; we expect a uniform, nonhierarchical barrier tree structure, shown in Figure 6.1(b) in the main text, for spin jam. The lack of hierarchical structure in this type of tree indicates that the weaker memory effect of spin jam results from a different mechanism. As the local minima in a spin jam result from the original zero energy mode of the classical spin Hamiltonian, it is plausible that the energy minima in the spin jam are grouped into clusters with different average barrier heights. This nonhierarchical tree resembles the so-called "banyan tree" pattern $[83,188]$. In this tree structure, different clusters are separated by a large barrier energy $T_{b}$, whereas the barrier energies within a cluster (labeled by $m$ ) are random numbers generated from an exponential distribution $p(\varepsilon)=e^{-\varepsilon / T_{m}} / T_{m}$. Here $T_{m}$ is an energy scale characterizing the local glassy transition for a cluster. This means that a system trapped in a cluster with energy $T_{m}$ will exhibit slow glassy dynamics when $T \lesssim T_{m}$. This energy scale $T_{m}$ varies from cluster to cluster and is assumed to be a random number uniformly distributed in the interval $T_{m} \in\left[0, T_{f}\right]$, where $T_{f}$ is the freezing temperature of the spin jam. Importantly, the energy scale for glass transition $T_{f}$ in a spin jam is determined by 
quantum fluctuations and is an order of magnitude smaller than the Curie-Weiss temperature. A similar random magnetization model is used here to describe the magnetic properties of a spin jam. Local minima within a cluster have a random magnetization $m$ uniformly distributed in the interval $\left[-M_{m},+M_{m}\right]$, where the bound $M_{m}$ itself is another random variable. Similar to the hierarchical tree counterpart, we assume a larger energy scale $T_{m}$ gives rise to a larger magnetization-bound $\mathrm{M}_{m}$.

We performed our Monte Carlo simulations following the same protocol as the experiments. The temperature decreases linearly during the cooling process, except the waiting-time period. Numerically, we start at an initial temperature of $1.5 T_{f}$, where $T_{f} \sim$ $T_{l=1}$ is the freezing temperature, and simulate cooling by decreasing the simulation temperature in small steps $\left(\Delta T \sim T_{f} / 3,300\right)$. When we reach base temperature, we heat the system up (rate of $\Delta T \sim T_{f} / 5000$ ). At each step, we perform 50 Monte Carlo updates. When there was waiting at an intermediate temperature, there were additional MC updates at the temperature while cooling, detailed in Figure (App.) C.2 (a) and (b), Insets. During the reheating part of the simulations, a small magnetic field $H$ is included to generate a finite magnetization. The dc susceptibility is simply $\chi_{D C}=M / H$. The numerical results shown in Fig. 4 of the main text were obtained for different waiting temperatures after averaging over $10^{5} \sim 10^{7}$ independent runs. Figure (App.) C.2 (a) and (b) shows the results obtained with different waiting times at $T_{w}=0.6 T_{f}$ for the spin jam and the spin glass model, respectively. Different curves in each panel correspond to varying numbers of MC steps that waited at $T_{w}$, which are proportional to the real waiting time $t_{w}$. Figure (App.) C.2 (c) (spin jam model) and (d) (spin glass model) shows $\left(M_{r e f}-M\right) / M_{r e f}$ as a function of (\# of MC steps)/10 that best reproduces the $t_{w}$ (in seconds) in the experiments, as shown in Figure 6.2 (d) in the main text. In Figure (App.) C.2 (c) and (d), ( $M_{r e f}-$ $M) / M_{\text {ref }}$ is rescaled so that their maximum values are 1. As shown in Figure (App.) C.2 (c) and (d), Insets at (\# of MC steps) $/ 10=10^{2},\left(M_{\text {ref }}-M\right) / M_{\text {ref }}$ of the spin glass model is almost twice that of the spin jam model. This difference for a short waiting time is consistent with our experimental observation (Figure 6.2 (a)-(c) in the main text). Furthermore, the overall dependence of $\left(M_{\text {ref }}-M\right) / M_{\text {ref }}$ as a function of $t_{w}$ reproduces our experimental data when scaled to the maximum value of $\left(M_{\text {ref }}-M\right) / M_{\text {ref }}$ data 
(Figure $6.2(\mathrm{~d})$ ). We note that our MC calculations based on the multilayer random energy model do not take into account other possible sources of magnetization. As a result, different scaling factors for $\left(M_{r e f}-M\right) / M_{\text {ref }}$ are required to reproduce the experimental data of different systems.

The memory effect in the hierarchical tree arises from the temperature-dependent relaxation dynamics. For a given waiting temperature $T_{w}$, the system will fluctuate over a small window of levels depending on $T_{l}$ and the population $\lambda_{l}$ of dead-end local minima at each level. A longer waiting time $t_{w}$ at this temperature thus allows the system to relax to a deeper and larger (entropically) valley of the energy surface. The memory effect observed during the reheating process results from the fact that the system is trapped in this special landscape basin. Similarly, the weaker memory effect in spin jam originates from the distribution of the cluster energy scales $T_{m}$. With decreasing temperature $T$, thermal equilibrium cannot be reached within clusters with $T_{m}>T$ as the corresponding relaxation dynamics become exponentially slow. The waiting temperature sets a threshold such that clusters with $T_{m}>T_{w}$ exhibit slow relaxation dynamics, whereas a longer waiting time $t_{w}$ helps the system find the cluster with lower overall energy and larger entropy. Again, the fact that the system is trapped in this special cluster manifests itself as the memory effect during rewarming. 

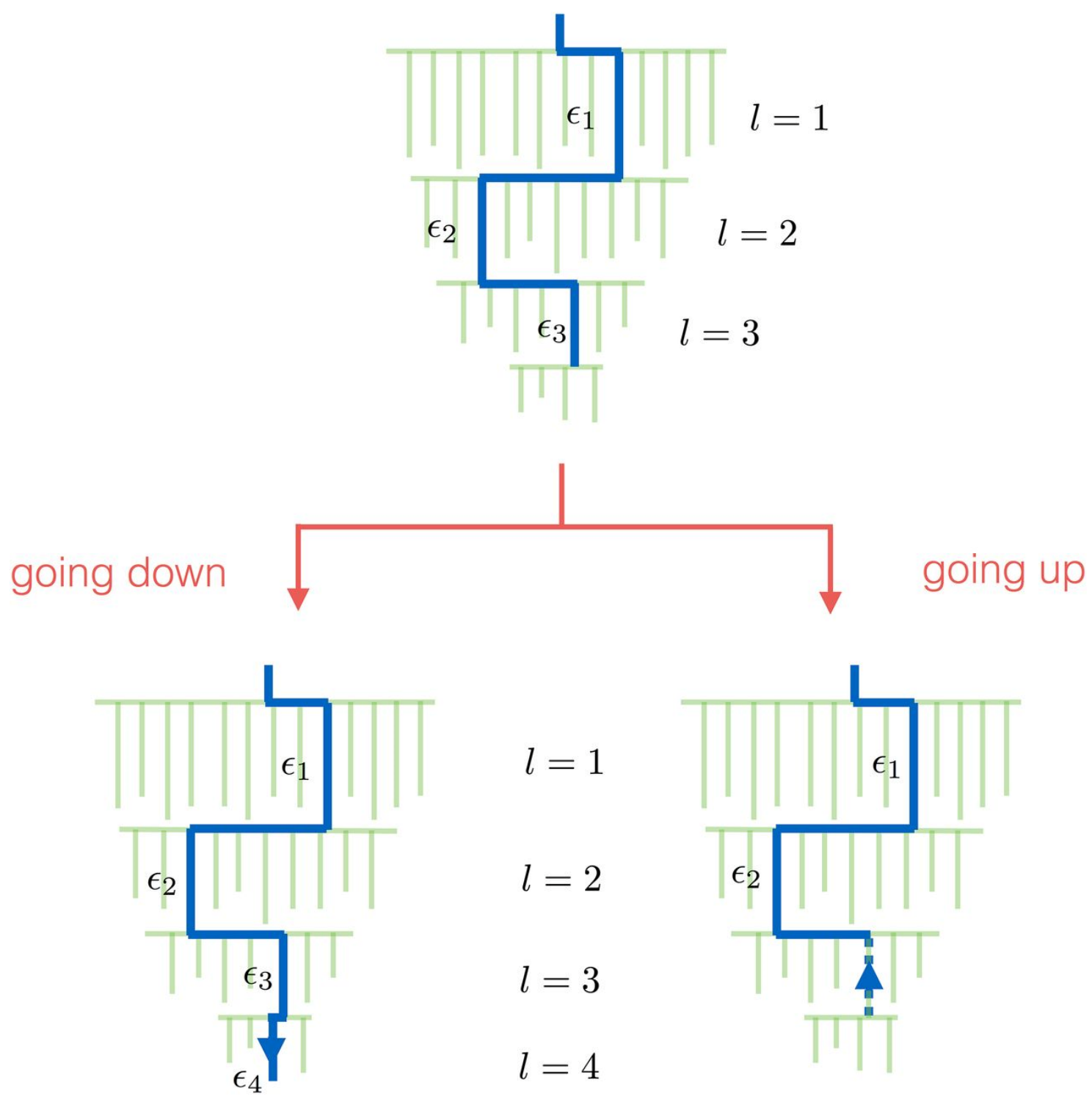

Figure (App.) C.1. Schematic diagram showing a Monte Carlo step in our dynamic barrier tree simulations. 

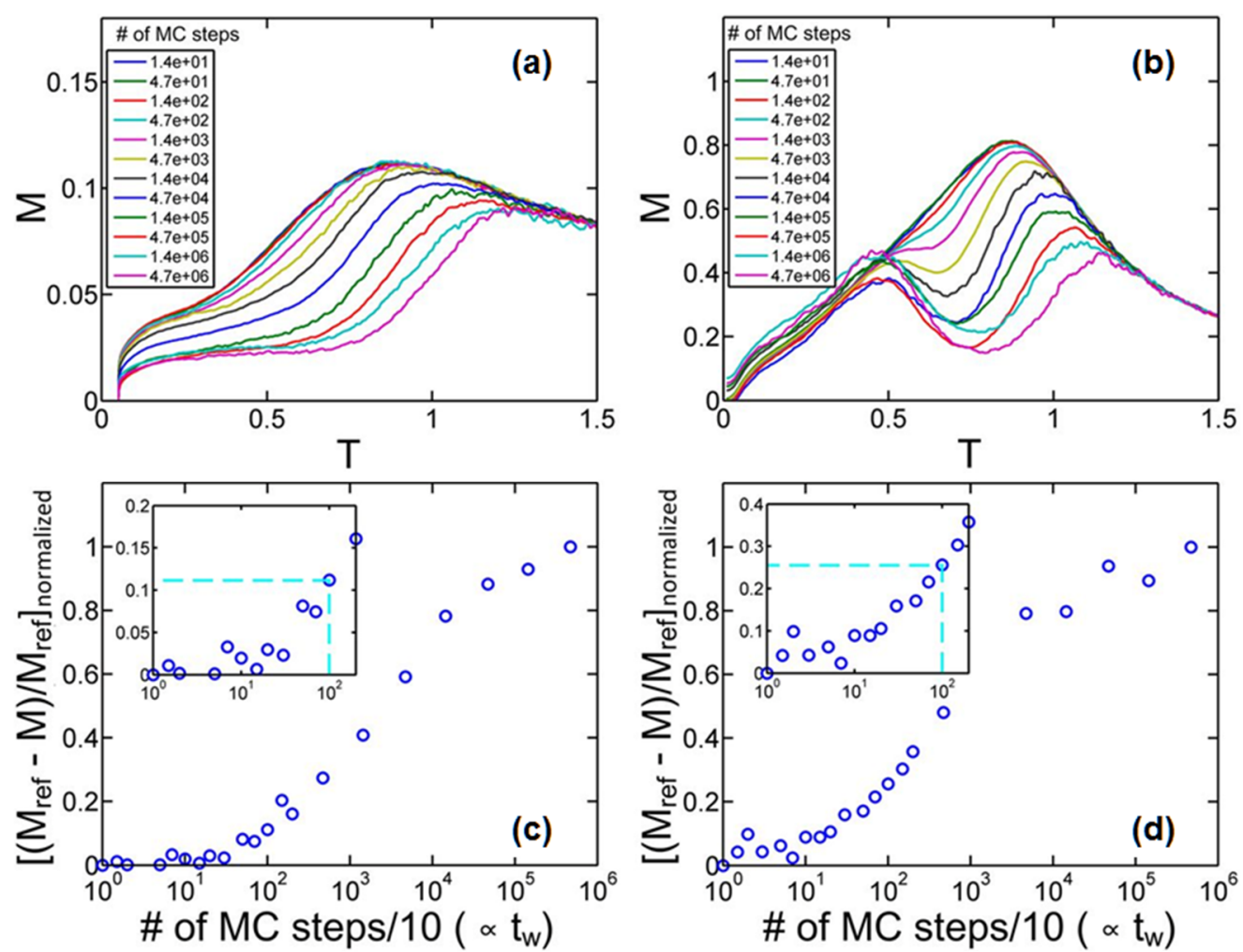

Figure (App.) C.2. Simulated Magnetization as a function of temperature.

[(a) and (b)] The magnetization $M$ as a function of temperature $T$ during heating for the spin jam and the spin glass model, respectively. Different curves in each panel correspond to varying numbers of MC steps that waited at $T_{w}$, which are proportional to $t_{w}$. (c) (spin jam model) and (d) (spin glass model) show $\left(M_{\text {ref }}-M\right) / M_{\text {ref }}$ as a function of (\# of MC steps)/ 10, which corresponds to tw (in seconds) in the experiments. The time scale has been chosen to best fit the experimental results. $\left(M_{\text {ref }}-M\right) / M_{\text {ref }}$ of both models are rescaled so that their maximum values are 1. As shown in (c) and (d), Insets, at (\# of MC steps) $/ 10=10^{2},\left(M_{\text {ref }}-M\right) / M_{\text {ref }}$ of the spin glass model is almost twice that of the spin jam model. 


\section{Appendix D \\ High Temperature susceptibility of $\mathrm{Fe}_{1.02} \mathrm{Se}_{0.15} \mathrm{Te}_{0.85}$ and $\mathrm{La}_{1.96} \mathrm{Sr}_{0.04} \mathrm{CuO}_{4}$}

The Figure S1 shows the DC susceptibility data obtained from $\mathrm{Fe}_{1.02} \mathrm{Se}_{0.15} \mathrm{Te}_{0.85}$ and $\mathrm{La}_{1.96} \mathrm{Sr}_{0.04} \mathrm{CuO}_{4}$ that exhibit their glassy transitions at low temperatures.
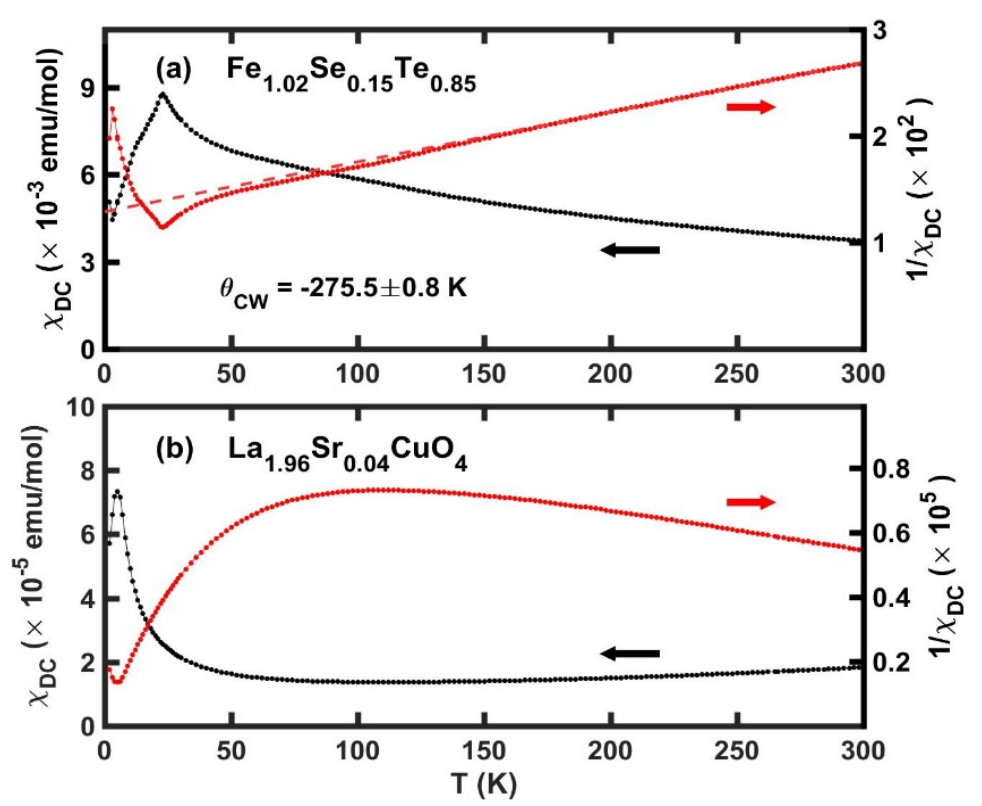

Figure (App.) D.1. High-Temperature bulk susceptibility of LSCO and FST samples.

High-Temperature bulk susceptibility (black) and inverse susceptibility (red) respectively, obtained from (a) $\mathrm{Fe}_{1.02} \mathrm{Se}_{0.15} \mathrm{Te}_{0.85}$ and (b) $\mathrm{La}_{1.96} \mathrm{Sr}_{0.04} \mathrm{CuO}_{4}$. The data above $120 \mathrm{~K}$ of $\mathrm{Fe}_{1.02} \mathrm{Se}_{0.15} \mathrm{Te}_{0.85}$ has been fitted to the CurieWeiss law (red dash line) and the estimated Curie-Weiss temperature is -265.5(8) K. The measurements have done under magnetic fields of $0.01 \mathrm{~T}$ and $0.1 \mathrm{~T}$ respectively. 


\section{Appendix E Polarization Analysis}

One can write down expression for the differential non-spin-flip and spin-flip crosssections in the various directions, in the presence of magnetic, nuclear and spin-incoherent scattering. As

$$
\begin{aligned}
& \left(\frac{\partial \sigma}{\partial \Omega}\right)_{x \downarrow}=\frac{1-p}{2} \cdot N+\frac{3+p}{6} \cdot I+\frac{1}{2} \cdot M \cdot\left(1+p \cdot \cos ^{2} \alpha\right) \\
& \left(\frac{\partial \sigma}{\partial \Omega}\right)_{x \uparrow}=\frac{1+p f}{2} \cdot N+\frac{3-p f}{6} \cdot I+\frac{1}{2} \cdot M \cdot\left(1-p f \cdot \cos ^{2} \alpha\right) \\
& \left(\frac{\partial \sigma}{\partial \Omega}\right)_{y \downarrow}=\frac{1-p}{2} \cdot N+\frac{3+p}{6} \cdot I+\frac{1}{2} \cdot M \cdot\left(1+p \cdot \sin ^{2} \alpha\right) \\
& \left(\frac{\partial \sigma}{\partial \Omega}\right)_{y \uparrow}=\frac{1+p f}{2} \cdot N+\frac{3-p f}{6} \cdot I+\frac{1}{2} \cdot M \cdot\left(1-p f \cdot \sin ^{2} \alpha\right) \\
& \left(\frac{\partial \sigma}{\partial \Omega}\right)_{z \downarrow}=\frac{1-p}{2} \cdot N+\frac{3+p}{6} \cdot I+\frac{1}{2} \cdot M \cdot(1+p) \\
& \left(\frac{\partial \sigma}{\partial \Omega}\right)_{z \uparrow}=\frac{1+p f}{2} \cdot N+\frac{3-p f}{6} \cdot I+\frac{1}{2} \cdot M \cdot(1-p f)
\end{aligned}
$$

where $\mathrm{M}, \mathrm{N}, \mathrm{I}$ are the magnetic $(\mathrm{M})$, nuclear coherent $(\mathrm{N})$ and nuclear spin-incoherent (I) intensities, respectively, $\mathrm{p}$ is the scaler beam polarization (assumed to be the same for all polarization settings), and $\mathrm{f}$ is the flipping efficiency. Here the flipper is on for the 'up' counts. The angle $\alpha$ is the angle between the $\mathrm{x}$-axis and the mean direction.

Note that this is the power average and won't hold, in general, for single crystal data. 


\section{Appendix F Linear Spin Waves for CN-ground States}

Author: S.-S. Zhang and C. D. Batista [192]

The ground state of the classical Kitaev model has an extensive degeneracy. The subset of $\mathrm{CN}$-ground states can be mapped to the close-packed dimer coverings of the honeycomb lattice. [154] The empty bonds of each dimer covering form self-avoiding (SW) paths, which are loops for closed boundary conditions. Within linear spinwave theory, magnons can only propagate along these $1 \mathrm{D}$ paths to lowest order in a $1 / S$ expansion. To compute the spin-wave Hamiltonian in each loop for a given $\mathrm{CN}$-ground state, it is convenient to use a twisted reference frame for the original Hamiltonian defined on a given loop, where the local Z-axis on a given site is chosen to be parallel to the spin direction:

$$
H_{1 D}=K \sum_{i=1}^{m}\left(\tilde{S}_{i 1}^{x} \tilde{S}_{i 2}^{x}+\tilde{S}_{i 2}^{y} \tilde{S}_{i+1,1}^{y}\right)-K S \sum_{i=1}^{m} \sum_{\alpha=1}^{2} \tilde{S}_{i \alpha}^{z}
$$

where $m=n / 2$ with $n$ being the number of sites on the loop. The local reference frame is chosen in such a way that two adjacent sites have the same local $x(y)$ axis if they are connected by a $x x(y y)$ bond. With this construction, the closed boundary condition can be periodic or anti-periodic depending on the direction of the last spin [154]. In this reference frame, the Hamiltonian is invariant under translations by two lattice sites. Correspondingly, the index $\alpha=1,2$ denotes the two sites on the effective unit cell. The second term of Equation (F.1) represents an effective perpendicular magnetic field generated by the adjacent $1 \mathrm{D}$ path through the antiferromagnetic interaction on the dimer. After a Holstein-Primakoff transformation, 


$$
\begin{aligned}
& \tilde{S}_{i \alpha}^{z}=S-a_{i \alpha}^{\dagger} a_{i \alpha} \\
& \tilde{S}_{i \alpha}^{x}=\frac{1}{\sqrt{2}}\left(a_{i \alpha}^{\dagger}+a_{i \alpha}\right), \quad \tilde{S}_{i \alpha}^{y}=\frac{1}{\sqrt{2 i}}\left(a_{i \alpha}^{\dagger}-a_{i \alpha}\right)
\end{aligned}
$$

the spin wave Hamiltonian (F.1) can be rewritten as

$$
\begin{aligned}
H_{S W} & =\frac{K S}{2} \sum_{i=1}^{m} \sum_{\alpha=1}^{2}\left(a_{i, \alpha}^{\dagger} a_{i, \alpha}+a_{i, \alpha} a_{i, \alpha}^{\dagger}\right) \\
& +\frac{K S}{2} \sum_{i=1}^{m}\left(a_{i, 1}^{\dagger} a_{i, 2}^{\dagger}+a_{i, 1}^{\dagger} a_{i, 2}+\text { h.c. }\right) \\
& -\frac{K S}{2} \sum_{i=1}^{m}\left(a_{i, 2}^{\dagger} a_{i+1,1}^{\dagger}+a_{i, 2}^{\dagger} a_{i+1,1}+\text { h.c. }\right)
\end{aligned}
$$

Given the translational symmetry of $H_{s w}$, we can diagonalize it by Fourier transforming the creation and annihilation operators:

$$
a_{i, \alpha}=\frac{1}{\sqrt{m}} \sum_{k} a_{k, \alpha} e^{i k\left(i+\delta_{\alpha}\right)}
$$

where $\delta_{\alpha}$ refers to the displacement within each unit cell. After Fourier transforming and applying a Bogoliubov transformation to $H_{S w}$, we obtain the diagonal form

$$
H_{s w}=\sum_{k} E(k) \beta_{k}^{\dagger} \beta_{k}+E_{0} \sum_{k} \gamma_{k}^{\dagger} \gamma_{k}+K S \sum_{k}\left|\cos \left(\frac{k}{2}\right)\right|
$$

where $E_{0}=0$. The branch of zero modes arises from the continuum of (non-CN) ground states connecting different $\mathrm{CN}$-ground states. The dispersion relation of the dispersive branch is [??]

$$
E(k)=2|K| S \cos (k / 2)
$$




\section{F.1 Dynamical Structure Factor}

To the lowest non-trivial order in the $1 / S$ expansion, the dynamical structure factor in momentum-frequency space for a given ground state, $|0\rangle$, has only contributions from the transverse spin components in the local reference frame

$$
\tilde{S}_{\alpha \beta}^{\mu \nu}(k, \omega)=4 \pi^{2} \sum_{n=1,2}\left\langle 0\left|\tilde{S}_{k, \alpha}^{\mu}\right| k, n\right\rangle\left\langle k, n\left|\tilde{S}_{-k, \beta}^{v}\right| 0\right\rangle \delta\left(\omega-E_{q, n}\right)
$$

As we explained in Chapter 8.1 , the local gauge structure of the Kitaev Hamiltonian, $\left[H_{K}, W_{p}\right]=0$, implies that the real space spin-spin correlators must vanish for distances bigger than one lattice parameter. Based on that observation, we will only compute the onsite and the NN spin-spin correlators that arise from taking the average over all the $\mathrm{CN}$ ground states. Note that a more rigorous calculation of the $T=0$ spin-spin correlator should also include the non-CN ground states. However, a calculation based on the just the $\mathrm{CN}$-ground states is enough to capture the main qualitative features of the dynamical structure factor obtained from our numerical simulations of the classical AFM Kitaev model.

Finally, given the critical nature of the dimer coverings of the honeycomb lattice, the loop length has a power law distribution, implying that most of the loops containing a given site (for the on-site correlator) and a pair of sites (for the two-site correlator) have a very long length. Consequently, we will assume that the average over $\mathrm{CN}$ states is dominated by the result for infinitely long loop length.

\section{F.2 On-site Dynamical Structure Factor}

The on-site dynamical structure factor is obtained by averaging over both sites of the unit cell of the loop:

$$
\tilde{S}_{0}^{x x}(\omega)=\frac{1}{2 m} \sum_{k}\left[\tilde{\chi}_{11}^{x x}(k, \omega)+\tilde{\chi}_{22}^{x x}(k, \omega)\right]
$$

Replacing the creation and annihilation operators of Holstein-Primakoff bosons with Bogoliubov bosons through 


$$
\left(\begin{array}{l}
a_{k, 1} \pm a_{-k, 1}^{\dagger} \\
a_{k, 2} \pm a_{-k, 2}^{\dagger}
\end{array}\right)=\left(\begin{array}{cc}
u_{k} & v_{k} \\
v_{k} & -u_{k}
\end{array}\right)^{(*)}\left(\begin{array}{c}
\gamma_{k} \pm \gamma_{-k}^{\dagger} \\
\beta_{k} \pm \beta_{-k}^{\dagger}
\end{array}\right)
$$

with

$$
u_{k}=\frac{i \sin \left(\frac{k}{4}\right)+\cos \left(\frac{k}{4}\right)}{\sqrt{2 \cos \left(\frac{k}{2}\right)}}, v_{k}=\frac{i \sin \left(\frac{k}{4}\right)-\cos \left(\frac{k}{4}\right)}{\sqrt{2 \cos \left(\frac{k}{2}\right)}}
$$

where the conjugation $(*)$ of the transformation matrix is taken for the "-" sign. After this substitution and taking the limit of $m \rightarrow \infty$, the on-site correlator is given by:

$$
\tilde{S}_{0}^{x x}(\omega)=\pi S \frac{\bar{\rho}\left(\frac{\omega}{K S}\right)}{\omega}+\frac{\pi S}{2} \delta(\omega) \int_{-\pi}^{\pi} \frac{d k}{\cos \left(\frac{k}{2}\right)}
$$

where the dimensionless density of states, $\bar{\rho}(\omega /(K S))=K S \rho(\omega)$, is defined as follows:

$$
\bar{\rho}(x)=\frac{2}{\sqrt{1-\left(\frac{x}{2}\right)^{2}}} .
$$

The divergence at $\omega=2 K S$ arises from the Van Hove singularity in the density of singlemagnon states at the top of the spin-wave band. Going back to the original reference frame, we have

$$
S_{0}(\omega)=\left\langle\tilde{S}_{0}^{x x}(\omega)\right\rangle
$$

where we do need not to specify the superscript because $S_{0}^{x x}(\omega)=S_{0}^{y y}(\omega)=S_{0}^{z z}(\omega)$.

\section{F.3 Nearest-neighbor Dynamical Structure Factor}

There are two different contributions to the dynamical spin correlator between nearestneighbor sites because of the two-site unit cell. Let us first consider the $x x$ bond $(i, 1)-$ $(i, 2)$. The conservation of the flux operators $W_{p}$ implies that only the correlator between the twisted $x$ spin components, $\left\langle\tilde{S}_{i, 1}^{x} \tilde{S}_{i, 2}^{x}\right\rangle$, is non-zero on this bond. From the spin wave theory, we have 


$$
\tilde{S}_{1}^{x x}(\omega) \simeq \frac{1}{m} \sum_{k} e^{-i k / 2}\left\langle\tilde{\chi}_{12}^{x x}(k, \omega)\right\rangle
$$

With

$$
\left\langle\tilde{\chi}_{12}^{x x}(k, \omega)\right\rangle=\frac{\pi^{2}}{\left|\cos \left(\frac{k}{2}\right)\right|}\left[e^{-i k / 2} \delta\left(\omega-E_{k}\right)-e^{-i k / 2} \delta(\omega)\right]
$$

In the $m \rightarrow \infty$ limit, there is

$$
\tilde{S}_{1}^{x x}(\omega)=\left[\frac{\pi \omega S}{2(K S)^{2}}-\frac{\pi S}{\omega}\right] \bar{\rho}\left(\frac{\omega}{K S}\right)-\frac{\pi S}{2} \delta(\omega) \int_{-\pi}^{\pi} \frac{d k}{\cos \left(\frac{k}{2}\right)}
$$

Similarly, only the y-components of the twisted spins, $\tilde{S}_{1}^{y y}(\omega)=\left\langle\tilde{S}_{i, 2}^{y} \tilde{S}_{i+1,1}^{y}\right\rangle$, contribute to the NN spin correlator on the other $y y$ bond $(i, 2)-(i+1 ; 1)$. By symmetry, this correlator is the same as the $x x$ correlator on the bond $(i, 1)-(i, 2)$ calculated above. Consequently, we can ignore the superscripts $x x / y y$ when referring to the $\mathrm{NN}$ spin correlator. Back to the original spin reference frame, we NN dynamic structure factor becomes

$$
S_{1}(\omega)=\tilde{S}_{1}^{x x}(\omega)
$$




\section{Appendix G Spin Dynamic Simulations}

\section{G.1 Sum Rules and Normalizations}

There are numerous sum rules exist, and they can be problem specific. For example, Let's consider an isotropic Kitaev model,

$$
S_{1}(\omega)=\sum_{p} \sum_{\alpha}^{ \pm x x \pm y y \pm z z} \sum_{l_{j k}}^{N_{\alpha}}\left\langle S_{l_{j}}^{p}(\omega)\right\rangle \times\left\langle S_{l_{k}}^{p}(\omega)\right\rangle^{\dagger}=6 \sum_{p} \sum_{l_{j k}}^{x x}\left\langle S_{l_{j}}^{p}(\omega)\right\rangle \times\left\langle S_{l_{k}}^{p}(\omega)\right\rangle^{\dagger}
$$

From the sum rules for Matter Fermions,

(1) $\int d \omega S^{\alpha}(\Gamma, \omega)=\left\langle S_{j}^{\alpha} S_{k}^{\alpha}\right\rangle+1 / 4$, where $\left\langle S_{j}^{\alpha} S_{k}^{\alpha}\right\rangle$-Nearest Neighbor equal time correlation function.

(2) $\int d \omega \omega S^{z z}(\Gamma, \omega)=\left(K_{x x}\left\langle S_{j}^{x x} S_{k}^{x x}\right\rangle+K_{y y}\left\langle S_{j}^{y y} S_{k}^{y y}\right\rangle\right) / 2$ and two other relations with $\alpha=x x, y y$ where $\left\langle S_{j}^{\alpha} S_{k}^{\alpha}\right\rangle$ is the nearest neighbor equal time correlation function,

$$
\left\langle S_{j}^{\alpha} S_{k}^{\alpha}\right\rangle=\sum_{t} \sum_{p} \sum_{\alpha}^{ \pm x x, \pm y y, \pm z z} \sum_{l_{j k}}^{N_{\alpha}}\left\langle S_{l_{j}}^{p}(t)\right\rangle \times\left\langle S_{l_{k}}^{p}(t)\right\rangle^{\dagger}
$$


For the isotropic Kitaev model, the Equation 2.25 can be rewritten as,

$$
\begin{gathered}
S(Q, \omega)=\sum_{p} \sum_{j k} \exp \left[-i Q\left(r_{j}-r_{k}\right)\right]\left\langle S_{j}^{p}(\omega)\right\rangle \times\left\langle S_{k}^{p}(\omega)\right\rangle^{\dagger} \\
S(Q, \omega)=S_{0}(\omega)+\sum_{p} \sum_{\alpha}^{ \pm x x, \pm y y, \pm z z} \sum_{l_{j k}}^{N_{\alpha}} \exp \left[-i Q R_{N N}^{\alpha}\right]\left\langle S_{l_{j}}^{p}(\omega)\right\rangle \times\left\langle S_{l_{k}}^{p}(\omega)\right\rangle^{\dagger} \\
S(Q, \omega)=S_{0}(\omega)+\sum_{\alpha}^{x x, y y, z z} 2 \cos \left[Q R_{N N}^{\alpha}\right] \sum_{p} \sum_{l_{j k}}^{N_{\alpha}}\left\langle S_{l_{j}}^{p}(\omega)\right\rangle \times\left\langle S_{l_{k}}^{p}(\omega)\right\rangle^{\dagger} \\
S(Q, \omega)=S_{0}(\omega)+S_{1}(\omega) \frac{1}{3} \sum_{\alpha}^{x x, y y, z z} \cos \left[Q R_{N N}^{\alpha}\right]
\end{gathered}
$$

However, for a particular bond type $(\alpha)$,

$$
S^{\alpha}(Q, \omega)=\frac{1}{3}\left(S_{0}(\omega)+S_{1}(\omega) \cos \left[Q R_{N N}^{\alpha}\right]\right)
$$

At the $\Gamma-$ point,

$$
S(\Gamma, \omega)=S_{0}(\omega)+S_{1}(\omega)
$$

From the rule (1),

$$
\int d \omega\left(S_{0}(\omega)+S_{1}(\omega)\right)-\sum_{\alpha}\left\langle S_{j}^{\alpha} S_{k}^{\alpha}\right\rangle=\frac{3}{4}
$$

Therefore, a normalization factor for $S(Q, \omega)$ can be calculated as,

$$
N=\frac{3 / 4}{\int d \omega\left(S_{0}(\omega)+S_{1}(\omega)\right)-\sum_{\alpha}\left\langle S_{j}^{\alpha} S_{k}^{\alpha}\right\rangle}
$$

Another way of normalizing the calculations can be using the neutron scattering sum rule,

$$
\int d \omega \int_{B Z} d Q S(Q, \omega)=S(S+1)
$$

By integrating over BZ, 


$$
\begin{gathered}
\int_{B Z} d Q S(Q, \omega)=V S_{0}(\omega)+W S_{1}(\omega) \\
V=\int_{B Z} d Q \quad W=\int_{B Z} d Q \frac{1}{3} \sum_{\alpha}^{x x, y y, z z} \cos \left[Q R_{N N}^{\alpha}\right]
\end{gathered}
$$

where $V$ and $W$ are constant. Thus,

$$
\int d \omega \int_{B Z} d Q S(Q, \omega)=V \int d \omega S_{0}(\omega)+W
$$

Therefore, a normalization factor for $S(Q, \omega)$ can be calculated as,

$$
N=\frac{S(S+1)}{V \int d \omega S_{0}(\omega)+W}
$$

The $V$ and $W$ for the Kitaev model can be found as $V=102.5 / R_{N N}$ and $W=-13.8 / R_{N N}$ respectively as estimated by Monte-Carlo sampling on a hexagon. In Chapter 8 , we have used the former normalization method in order to compare with the quantum solution.

Since we ignore the Planck constant $(\hbar)$ and Boltzmann constant $\left(k_{B}\right)$ throughout the spindynamic simulations and subsequent calculations, we have to normalize MC-time and the corresponding energy transfer $(\hbar \omega)$ as $t=\frac{\hbar}{k_{B}} t_{M C}$ and $\hbar \omega=2 \pi k_{B} \omega_{M C}$ where $\omega_{M C}=$ $1 / t_{M C}$. Here $t$ is in nanoseconds (ns) and $\hbar \omega$ in millielectron volts (meV). 


\section{G.2 Simple Heisenberg models in Square Lattice}

$S(Q, \omega)$ for simple nearest neighbor Heisenberg models on a simple square lattice, has been calculated as a sanity check and compared with Linear Spinwave Theory (LST) as shown in the Figure (App.) G.1. For LST calculation, we have used SpinW program from Paul Scherrer Institute (PSI).
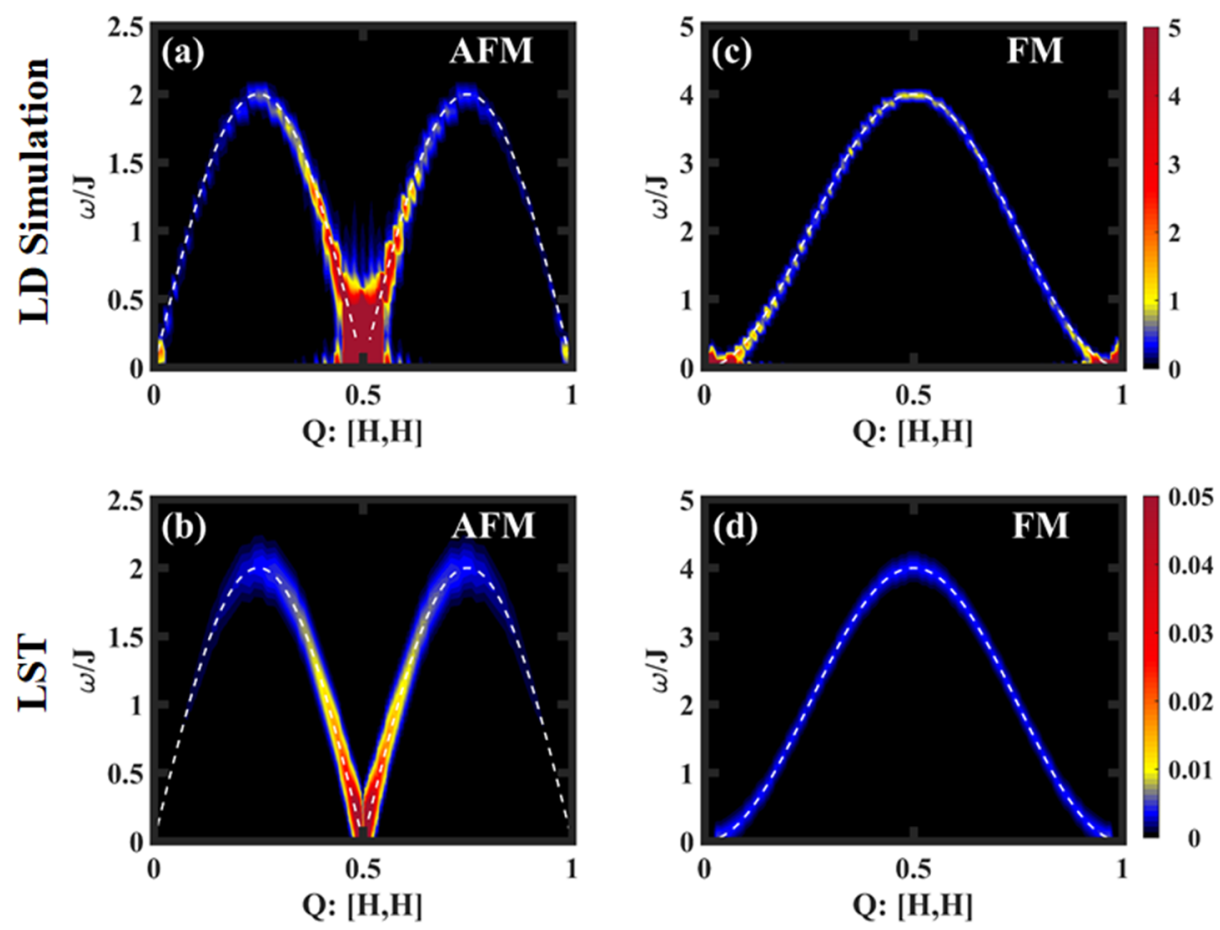

Figure (App.) G.1. Dispersion relations of simple Heisenberg square lattices

Sanity checks on $S(Q, \omega)$ of simple AFM and FM Heisenberg models on a simple square lattice. The calculation from (a), (c) dynamic simulations (LD) and (b),(d) Linear Spinwave Theory (LPT) are compared. The corresponding spinwave dispersions are overpotted as white dash lines. 


\section{Appendix $\mathrm{H}$ Important Relations}

* $E=81.804 / \lambda^{2}=2.072 \mathrm{~K}_{i}^{2} ; \quad E$ in $\mathrm{meV}, \quad \lambda$ in $\AA, \mathrm{K}_{i}$ in $\AA^{-1}$

* $E_{\text {thermal }}=0.086 \times T ; \quad E_{\text {thermal }}$ in $\mathrm{meV}, T$ in $\mathrm{K}$

* $E=4 \times 10^{-3} / t=4 / t^{\prime} ; t$ in ns, $t^{\prime}$ in ps

* $E=\left(g_{s} . S\right) \times 0.058 \times B ; E$ in meV, $B$ in Tesla (T)

* Curie-Weiss Law, $\chi=C /\left(T-\theta_{C W}\right)$

Curie constant, $C=N g^{2} J(J+1) \mu_{0} \mu_{B}^{2} / 3 k_{B}$

Curie-Weiss Temperature, $\theta_{C W}=N g^{2} J(J+1) \lambda \mu_{B}^{2} / 3 k_{B}$

Molecular Field, $\lambda=\frac{\partial H}{\partial S_{i}} ; H-$ microscopic hamiltonian

* From Bragg's Law, $Q=(4 \pi \sin (\theta)) / \lambda ; \lambda$ - Wave Length of incident beam, in A. $\quad \theta=\alpha / 2$ where $\alpha$ is the scattering angle. 


\section{Bibliography}

[1] M. E. Cates, J. P. Wittmer, J. Bouchaud, and P. Claudin, Phys. Rev. Lett. 81, 1841 (1998).

[2] A. J. Liu and S. R. Nagel, Nature 396, 21 (1998).

[3] M. P. Marder, Condensed matter physics (John Wiley \& Sons, 2010).

[4] K. Iida, S. Lee, and S. Cheong, Phys. Rev. Lett. 108, 217207 (2012).

[5] I. Klich, S. Lee, and K. Iida, Nature communications 5 (2014).

[6] J. Bland, www.cmp.liv.ac.uk/frink/thesis/thesis/node71.html.

[7] J. A. Mydosh, Spin glasses: an experimental introduction (Taylor and Francis, 1993).

[8] D. L. Stein and C. M. Newman, Physical Review E 51, 5228 (1995).

[9] T. Elovaara, H. Huhtinen, S. Majumdar, and P. Paturi, Journal of Physics: Condensed Matter 24, 216002 (2012).

[10] S. Mukherjee, A. K. Pal, S. Bhattacharya, and J. Raittila, Physical Review B 74, 104413 (2006).

[11] P. A. Joy, P. A. Kumar, and S. K. Date, Journal of Physics: Condensed Matter 10, 11049 (1998).

[12] J. Villain, R. Bidaux, J. P. Carton, and R. Conte, J. Phys. France, 1263 (1980).

[13] A. Chubukov, Phys. Rev. Lett. 69, 832 (1992).

[14] C. L. Henley, Phys. Rev. Lett. 62, 2056 (1989).

[15] S. Sachdev, Physical Review B 45, 12377 (1992).

[16] B. I. Halperin and W. M. Saslow, Physical Review B 16, 2154 (1977). 
[17] P. W. Anderson, B. I. Halperin, and C. M. Varma, Philosophical Magazine 25, 1 (1972).

[18] E. Clementyev, Magnetic Susceptibility: Basic principles and features of the PPMS, 2004).

[19] M. Gingras, C. V. Stager, N. P. Raju, B. D. Gaulin, and J. E. Greedan, Phys. Rev. Lett. 78, 947 (1997).

[20] J. S. Gardner, B. D. Gaulin, S. Lee, C. Broholm, N. P. Raju, and J. E. Greedan, Phys. Rev. Lett. 83, 211 (1999).

[21] N. J. Carron, An introduction to the passage of energetic particles through matter (CRC Press, 2006).

[22] J. Copley and J. C. Cook, Chem. Phys. 292, 477 (2003).

[23] G. L. Squires, Introduction to the theory of thermal neutron scattering (Cambridge university press, 2012).

[24] S. W. Lovesey, (1984).

[25] R. F. Bryan, International tables for crystallography.Vol.C.Mathematical, physical and chemical tables edited by AJC Wilson (1993).

[26] E. Mamontov and K. W. Herwig, Rev. Sci. Instrum. 82, 085109 (2011).

[27] A. Meyer, R. M. Dimeo, P. M. Gehring, and D. A. Neumann, Rev. Sci. Instrum. 74, $2759(2003)$.

[28] M. Birr, A. Heidemann, and B. Alefeld, Nucl. Instrum. Methods 95, 435 (1971).

[29] J. C. Cook, W. Petry, A. Heidemann, and J. F. Barthlemy, Nuclear Instruments and Methods in Physics Research Section A: Accelerators, Spectrometers, Detectors and Associated Equipment 312, 553 (1992). 
[30] F. Mezei, Zeitschrift fr Physik A Hadrons and nuclei 255, 146 (1972).

[31] U. Atxitia, O. Chubykalo-Fesenko, R. W. Chantrell, U. Nowak, and A. Rebei, Phys. Rev. Lett. 102, 057203 (2009).

[32] A. P. Ramirez, G. P. Espinosa, and A. S. Cooper, Phys. Rev. Lett. 64, 2070 (1990).

[33] S. Lee, C. Broholm, G. Aeppli, A. P. Ramirez, T. G. Perring, C. J. Carlile, M.

Adams, T. Jones, and B. Hessen, EPL (Europhysics Letters) 35, 127 (1996).

[34] S. Lee, C. Broholm, G. Aeppli, T. G. Perring, B. Hessen, and A. Taylor, Phys. Rev. Lett. 76, 4424 (1996).

[35] X. Obradors, A. Labarta, A. Isalgue, J. Tejada, J. Rodriguez, and M. Pernet, Solid State Commun. 65, 189 (1988).

[36] C. Broholm, G. P. Espinosa, A. S. Cooper, and G. Aeppli, Journal of Applied Physics 67, 5799 (1990).

[37] A. P. Ramirez, B. Hessen, and M. Winklemann, Phys. Rev. Lett. 84, 2957 (2000).

[38] A. Keren, Y. J. Uemura, G. Luke, P. Mendels, M. Mekata, and T. Asano, Phys. Rev. Lett. 84, 3450 (2000).

[39] H. Mutka, G. Ehlers, C. Payen, D. Bono, J. R. Stewart, P. Fouquet, P. Mendels, J. Y. Mevellec, N. Blanchard, and G. Collin, Phys. Rev. Lett. 97, 047203 (2006).

[40] P. Mendels, A. Keren, L. Limot, M. Mekata, G. Collin, and M. Horvatić, Phys. Rev. Lett. 85, 3496 (2000).

[41] L. Limot, P. Mendels, G. Collin, C. Mondelli, B. Ouladdiaf, H. Mutka, N. Blanchard, and M. Mekata, Physical Review B 65, 144447 (2002).

[42] D. Bono, P. Mendels, G. Collin, and N. Blanchard, Phys. Rev. Lett. 92, 217202 (2004). 
[43] D. Bono, P. Mendels, G. Collin, N. Blanchard, F. Bert, A. Amato, C. Baines, and A. D. Hillier, Phys. Rev. Lett. 93, 187201 (2004).

[44] I. S. Hagemann, Q. Huang, X. Gao, A. P. Ramirez, and R. J. Cava, Phys. Rev. Lett. 86, 894 (2001).

[45] B. Martinez, F. Sandiumenge, A. Rouco, A. Labarta, J. Rodríguez-Carvajal, M.

Tovar, M. T. Causa, S. Gali, and X. Obradors, Physical Review B 46, 10786 (1992).

[46] D. Bono, L. Limot, P. Mendels, G. Collin, and N. Blanchard, Low Temp. Phys. 31, 704 (2005).

[47] J. Yang, A. M. Samarakoon, K. W. Hong, J. R. Copley, Q. Huang, A. Tennant, T. J. Sato, and S. Lee, Journal of the Physical Society of Japan 85, 094712 (2016).

[48] P. Bonnet, C. Payen, H. Mutka, M. Danot, P. Fabritchnyi, J. R. Stewart, A. Mellergård, and C. Ritter, Journal of Physics: Condensed Matter 16 (2004).

[49] S. Lee, C. Broholm, G. Aeppli, A. P. Ramirez, T. G. Perring, C. J. Carlile, M. Adams, T. Jones, and B. Hessen, EPL (Europhysics Letters) 35, 127 (1996). [50] P. Schiffer, A. P. Ramirez, K. N. Franklin, and S. W. Cheong, Phys. Rev. Lett. 77, 2085 (1996).

[51] J. Copley and J. C. Cook, Chem. Phys. 292, 477 (2003).

[52] R. T. Azuah, L. R. Kneller, Y. Qiu, P. L. Tregenna-Piggott, C. M. Brown, J. R. Copley, and R. M. Dimeo, Journal of Research of the National Institute of Standards and Technology 114, 341 (2009).

[53] S. W. Lovesey, (1984).

[54] A. Furrer and H. U. Gdel, J Magn Magn Mater 14, 256 (1979).

[55] R. H. Heffner and D. E. MacLaughlin, Physical Review B 29, 6048 (1984). 
[56] F. Mezei and A. P. Murani, J Magn Magn Mater 14, 211 (1979).

[57] F. Mezei, Physica B C 120, 51 (1983).

[58] R. Moessner and A. J. Berlinsky, Phys. Rev. Lett. 83, 3293 (1999).

[59] A. Murani, J Magn Magn Mater 22, 271 (1981).

[60] D. Podolsky and Y. B. Kim, Physical Review B 79, 140402 (2009).

[61] A. P. Ramirez, G. P. Espinosa, and A. S. Cooper, Physical Review B 45, 2505 (1992).

[62] E. J. Samuelsen, M. T. Hutchings, and G. Shirane, Physica 48, 13 (1970).

[63] C. L. Henley, Can. J. Phys. 79, 1307 (2001).

[64] H. J. Silverstein, K. Fritsch, F. Flicker, A. M. Hallas, J. S. Gardner, Y. Qiu, G.

Ehlers, A. T. Savici, Z. Yamani, and K. A. Ross, Physical Review B 89, 054433 (2014).

[65] H. Shinaoka, Y. Motome, T. Miyake, and S. Ishibashi, Physical Review B 88, 174422 (2013).

[66] S. Lee, D. Louca, H. Ueda, S. Park, T. J. Sato, M. Isobe, Y. Ueda, S. Rosenkranz, P. Zschack, and J. iguez, Phys. Rev. Lett. 93, 156407 (2004).

[67] M. Gingras, C. V. Stager, B. D. Gaulin, N. P. Raju, and J. E. Greedan, J. Appl. Phys. 79, 6170 (1996).

[68] C. Chamon, Phys. Rev. Lett. 94, 040402 (2005).

[69] W. De Roeck and F. Huveneers, Communications in Mathematical Physics 332, 1017 (2014).

[70] C. P. Goodrich, A. J. Liu, and S. R. Nagel, Nature Physics 10, 578 (2014).

[71] G. Tarjus, S. A. Kivelson, Z. Nussinov, and P. Viot, Journal of Physics: Condensed Matter 17 (2005). 
[72] J. Yang, A. Samarakoon, S. Dissanayake, H. Ueda, I. Klich, K. Iida, D. Pajerowski, N. P. Butch, Q. Huang, and J. R. Copley, Proceedings of the National Academy of Sciences 112, 11519 (2015).

[73] D. Hser, L. E. Wenger, A. J. Van Duyneveldt, and J. A. Mydosh, Physical Review B 27, 3100 (1983).

[74] V. K. Anand, D. T. Adroja, and A. D. Hillier, Physical Review B 85, 014418 (2012).

[75] M. Balanda, Acta Physica Polonica A 124, 964 (2013).

[76] K. D. Ball, R. S. Berry, R. E. Kunz, F. Li, A. Proykova, and D. J. Wales, Science, 963 (1996).

[77] P. G. Debenedetti and F. H. Stillinger, Nature 410, 259 (2001).

[78] C. A. Angell, Science 267, 1924 (1995).

[79] A. J. Kovacs, J. J. Aklonis, J. M. Hutchinson, and A. R. Ramos, Journal of Polymer Science Part B: Polymer Physics 17, 1097 (1979).

[80] T. Watanabe, N. Masuda, F. Megumi, R. Kanai, and G. Rees, Nature communications 5 (2014).

[81] S. A. Marvel, S. H. Strogatz, and J. M. Kleinberg, Phys. Rev. Lett. 103, 198701 (2009).

[82] W. Janke, Rugged free energy landscapes: Common computational approaches to spin glasses, structural glasses and biological macromolecules (Springer, 2007), 736.

[83] D. J. Wales, M. A. Miller, and T. R. Walsh, Nature 394, 758 (1998).

[84] J. D. Bryngelson and P. G. Wolynes, Proceedings of the National Academy of Sciences 84, 7524 (1987). 
[85] B. Fain and M. Levitt, Proceedings of the National Academy of Sciences 100, 10700 (2003).

[86] J. Dolinšek, Z. Jagličić, T. J. Sato, J. Q. Guo, and A. P. Tsai, Journal of Physics:

Condensed Matter 15, 7981 (2003).

[87] H. Mamiya, N. Tsujii, N. Terada, S. Nimori, H. Kitazawa, A. Hoshikawa, and T.

Ishigaki, Physical Review B 90, 014440 (2014).

[88] H. Mamiya and S. Nimori, J. Appl. Phys. 111 (2012).

[89] F. Ladieu, F. Bert, V. Dupuis, E. Vincent, and J. Hammann, Journal of Physics:

Condensed Matter 16 (2004).

[90] V. Dupuis, F. Bert, J. -. Bouchaud, J. Hammann, F. Ladieu, D. Parker, and E.

Vincent, Pramana 64, 1109 (2005).

[91] V. Dupuis, E. Vincent, J. Hammann, J. E. Greedan, and A. S. Wills, J. Appl. Phys. 91, 8384 (2002).

[92] F. Krzakala and F. Ricci-Tersenghi, in Journal of Physics: Conference Series (IOP Publishing, 2006), p. 42.

[93] L. Zou and S. R. Nagel, Phys. Rev. Lett. 104, 257201 (2010).

[94] M. Sasaki and K. Nemoto, Journal of the Physical Society of Japan 69, 2283 (2000).

[95] O. M. Becker and M. Karplus, J. Chem. Phys. 106, 1495 (1997).

[96] P. Garstecki, T. X. Hoang, and M. Cieplak, Physical Review E 60, 3219 (1999).

[97] D. Sherrington and S. Kirkpatrick, Phys. Rev. Lett. 35, 1792 (1975).

[98] L. Rademaker, Z. Nussinov, L. Balents, and V. Dobrosavljevic, arXiv preprint arXiv:1605.01822 (2016). 
[99] D. Gfeller, P. De Los Rios, A. Caflisch, and F. Rao, Proceedings of the National Academy of Sciences 104, 1817 (2007).

[100] J. P. Doye, Phys. Rev. Lett. 88, 238701 (2002).

[101] R. Albert and A. Barabsi, Reviews of modern physics 74, 47 (2002).

[102] J. A. Mydosh, Spin glasses: an experimental introduction (Taylor and Francis, 1993).

[103] V. Cannella and J. A. Mydosh, Physical Review B 6, 4220 (1972).

[104] P. W. Anderson, Physics Today, 9 (1988).

[105] S. Wakimoto, R. J. Birgeneau, M. A. Kastner, Y. S. Lee, R. Erwin, P. M. Gehring, S. H. Lee, M. Fujita, K. Yamada, and Y. Endoh, Physical Review B 61, 3699 (2000). [106] M. Matsuda, M. Fujita, K. Yamada, R. J. Birgeneau, M. A. Kastner, H. Hiraka, Y. Endoh, S. Wakimoto, and G. Shirane, Physical Review B 62, 9148 (2000).

[107] N. Katayama, S. Ji, D. Louca, S. Lee, M. Fujita, T. J. Sato, J. Wen, Z. Xu, G. Gu, and G. Xu, Journal of the Physical Society of Japan 79, 113702 (2010).

[108] Y. Luo, C. Cao, B. Si, Y. Li, J. Bao, H. Guo, X. Yang, C. Shen, C. Feng, and J. Dai, Physical Review B 87, 161121 (2013).

[109] P. Khuntia, S. Manni, F. R. Foronda, T. Lancaster, S. J. Blundell, P. Gegenwart, and M. Baenitz, arXiv preprint arXiv:1512.04904 (2015).

[110] S. Manni, Y. Tokiwa, and P. Gegenwart, Physical Review B 89, 241102 (2014).

[111] G. Aeppli, S. M. Hayden, H. A. Mook, Z. Fisk, S. Cheong, D. Rytz, J. P. Remeika, G. P. Espinosa, and A. S. Cooper, Phys. Rev. Lett. 62, 2052 (1989).

[112] R. Coldea, S. M. Hayden, G. Aeppli, T. G. Perring, C. D. Frost, T. E. Mason, S. Cheong, and Z. Fisk, Phys. Rev. Lett. 86, 5377 (2001). 
[113] W. Bao, Y. Qiu, Q. Huang, M. A. Green, P. Zajdel, M. R. Fitzsimmons, M.

Zhernenkov, S. Chang, M. Fang, and B. Qian, Phys. Rev. Lett. 102, 247001 (2009).

[114] P. Dai, J. Hu, and E. Dagotto, arXiv preprint arXiv:1209.0381 (2012).

[115] V. M. Katukuri, S. Nishimoto, I. Rousochatzakis, H. Stoll, J. Van Den Brink, and L. Hozoi, Scientific reports 5 (2015).

[116] S. K. Choi, R. Coldea, A. N. Kolmogorov, T. Lancaster, I. I. Mazin, S. J. Blundell, P. G. Radaelli, Y. Singh, P. Gegenwart, and K. R. Choi, Phys. Rev. Lett. 108, 127204 (2012).

[117] R. V. Chamberlin, G. Mozurkewich, and R. Orbach, Phys. Rev. Lett. 52, 867 (1984).

[118] R. M. Roshko and W. Ruan, J Magn Magn Mater 104, 1613 (1992).

[119] P. Mitchler, R. M. Roshko, and W. Ruan, Journal de Physique I 2, 2299 (1992).

[120] R. G. Palmer, D. L. Stein, E. Abrahams, and P. W. Anderson, Phys. Rev. Lett. 53, 958 (1984).

[121] M. A. Continentino and A. P. Malozemoff, Physical Review B 33, 3591 (1986).

[122] I. A. Campbell, Physical Review B 37, 9800 (1988).

[123] A. T. Ogielski, Physical Review B 32, 7384 (1985).

[124] A. Samarakoon, T. J. Sato, T. Chen, G. Chern, J. Yang, I. Klich, R. Sinclair, H. Zhou, and S. Lee, Proceedings of the National Academy of Sciences 113, 11806 (2016). [125] H. Shinaoka, Y. Motome, T. Miyake, and S. Ishibashi, Physical Review B 88, 174422 (2013).

[126] D. S. Fisher and D. A. Huse, Physical Review B 38, 386 (1988). 
[127] S. Cheong, G. Aeppli, T. E. Mason, H. Mook, S. M. Hayden, P. C. Canfield, Z.

Fisk, K. N. Clausen, and J. L. Martinez, Phys. Rev. Lett. 67, 1791 (1991).

[128] V. J. Emery, S. A. Kivelson, and J. M. Tranquada, Proceedings of the National Academy of Sciences 96, 8814 (1999).

[129] S. Lee, G. Xu, W. Ku, J. S. Wen, C. C. Lee, N. Katayama, Z. J. Xu, S. Ji, Z. W. Lin, and G. D. Gu, Physical Review B 81, 220502 (2010).

[130] T. J. Liu, J. Hu, B. Qian, D. Fobes, Z. Q. Mao, W. Bao, M. Reehuis, S. Kimber, K. Prokes, and S. Matas, arXiv preprint arXiv:1003.5647 (2010).

[131] J. Bouchaud, V. Dupuis, J. Hammann, and E. Vincent, Physical review B 65, 024439 (2001).

[132] M. Lederman, R. Orbach, J. M. Hammann, M. Ocio, and E. Vincent, Physical Review B 44, 7403 (1991).

[133] J. F. Fontanari and P. F. Stadler, Journal of Physics A: Mathematical and General 35, 1509 (2002).

[134] P. Charbonneau, J. Kurchan, G. Parisi, P. Urbani, and F. Zamponi, arXiv preprint arXiv:1404.6809 (2014).

[135] S. Franz, G. Parisi, P. Urbani, and F. Zamponi, Proceedings of the National Academy of Sciences 112, 14539 (2015).

[136] K. Chen, W. G. Ellenbroek, Z. Zhang, D. T. Chen, P. J. Yunker, S. Henkes, C.

Brito, O. Dauchot, W. Van Saarloos, and A. J. Liu, Phys. Rev. Lett. 105, 025501 (2010).

[137] A. J. Liu and S. R. Nagel, Annu.Rev.Condens.Matter Phys. 1, 347 (2010).

[138] A. Kitaev, 321, 2 (2006).

[139] G. Jackeli and G. Khaliullin, 102, 017205 (2009). 
[140] Banerjee, A and Bridges, CA and Yan, J-Q and Aczel, AA and Li, L and Stone, MB and Granroth, GE and Lumsden, MD and Yiu, Y and Knolle, J and others, 15, 733 (2016).

[141] M. Gohlke, R. Verresen, R. Moessner, and F. Pollmann, (2017).

[142] D. Gotfryd, J. Rusna vcko, K. Wohlfeld, G. Jackeli, Chaloupka, Ji vr ' i, and M. Ole 's Andrzej, 95, 024426 (2017).

[143] J. Knolle, D. L. Kovrizhin, J. T. Chalker, and R. Moessner, 112, 207203 (2014).

[144] J. Knolle, D. L. Kovrizhin, J. T. Chalker, and R. Moessner, 92, 115127 (2015).

[145] J. Yoshitake, J. Nasu, and Y. Motome, Phys. Rev. Lett. 117, 157203 (2016).

[146] J. Yoshitake, J. Nasu, Y. Kato, and Y. Motome, .

[147] Y. J., N. J., and M. Y., .

[148] Do, Seung-Hwan and Park, Sang-Youn and Yoshitake, Junki and Nasu, Joji and Motome, Yukitoshi and Kwon, Yong Seung and Adroja, DT and Voneshen, DJ and Kim, Kyoo and Jang, T-H and others, (2017).

[149] A. Banerjee, J. Yan, J. Knolle, C. A. Bridges, M. B. Stone, M. D. Lumsden, D. G. Mandrus, D. A. Tennant, R. Moessner, and S. E. Nagler, 356, 1055 (2017).

[150] T. Huberman, D. A. Tennant, R. A. Cowley, R. Coldea, and C. D. Frost, 2008 (2008).

[151] J. Nasu, M. Udagawa, and Y. Motome, 113, 197205 (2014).

[152] J. Nasu, M. Udagawa, and Y. Motome, 92, 115122 (2015).

[153] G. Baskaran, S. Mandal, and R. Shankar, 98, 247201 (2007).

[154] G. Baskaran, D. Sen, and R. Shankar, 78, 115116 (2008).

[155] S. Chandra, K. Ramola, and D. Dhar, 82, 031113 (2010). 
[156] I. Rousochatzakis, Y. Sizyuk, and N. B. Perkins, .

[157] C. C. Price and N. B. Perkins, 109, 187201 (2012).

[158] C. Price and N. B. Perkins, 88, 024410 (2013).

[159] I. Rousochatzakis and N. B. Perkins, 118, 147204 (2017).

[160] J. Nasu, Y. Kato, J. Yoshitake, Y. Kamiya, and Y. Motome, 118, 137203 (2017).

[161] G. Khaliullin, 160, 155 (2005).

[162] Chaloupka, Ji vr ' i, G. Jackeli, and G. Khaliullin, 105, 027204 (2010).

[163] Chaloupka, Ji vr ' i, G. Jackeli, and G. Khaliullin, 110, 097204 (2013).

[164] J. '. Kondev, J. d. Gier, and B. Nienhuis, 29, 6489 (1996).

[165] Bl ote, H W J and B. Nienhuis, 72, 1372 (1994).

[166] X. Song, Y. You, and L. Balents, 117, 037209 (2016).

[167] S. Lin, Y. Kamiya, G. Chern, and C. D. Batista, 112, 155702 (2014).

[168] A. Wei sse, G. Wellein, A. Alvermann, and H. Fehske, 78, 275 (2006).

[169] J. Yoshitake, J. Nasu, and Y. Motome, 117, 157203 (2016).

[170] R. Yadav, N. A. Bogdanov, V. M. Katukuri, S. Nishimoto, J. van den Brink, and L.

Hozoi, 6 (2016).

[171] S. M. Winter, Y. Li, H. O. Jeschke, and R. Valenti, 93, 214431 (2016).

[172] W. Wang, Z. Dong, S. Yu, and J. Li, (2016).

[173] H. Kim and H. Kee, 93, 155143 (2016).

[174] H. Kim, V. S. V., A. Catuneanu, and H. Kee, 91, 241110 (2015).

[175] Y. Sizyuk, P. W olfle, and N. B. Perkins, 94, 085109 (2016).

[176] B. H. Kim, T. Shirakawa, and S. Yunoki, 117, 187201 (2016).

[177] Chaloupka, Ji vr ' i and G. Khaliullin, 94, 064435 (2016). 
[178] L. Janssen, E. C. Andrade, and M. Vojta, 117, 277202 (2016).

[179] Y. S. Hou, H. J. Xiang, and X. G. Gong, (2016).

[180] S. M. Winter, K. Riedl, A. Honecker, and R. Valenti, (2017).

[181] Ran, Kejing and Wang, Jinghui and Wang, Wei and Dong, Zhao-Yang and Ren, Xiao and Bao, Song and Li, Shichao and Ma, Zhen and Gan, Yuan and Zhang, Youtian and others, 118, 107203 (2017).

[182] E. Sela, H. Jiang, M. H. Gerlach, and S. Trebst, 90, 035113 (2014).

[183] Choi, SK and Coldea, R and Kolmogorov, AN and Lancaster, T and Mazin, II and Blundell, SJ and Radaelli, PG and Singh, Yogesh and Gegenwart, P and Choi, KR and others, 108, 127204 (2012).

[184] A. C. Larson and R. B. Von Dreele, General Structure Analysis System.LANSCE, MS-H805, Los Alamos, New Mexico (1994).

[185] S. Chen, H. Guo, K. A. Seu, K. Dumesnil, S. Roy, and S. K. Sinha, Phys. Rev. Lett. 110, 217201 (2013).

[186] A. Samarakoon, T. J. Sato, T. Chen, G. Chern, J. Yang, I. Klich, R. Sinclair, H. Zhou, and S. Lee, Proceedings of the National Academy of Sciences 113, 11806 (2016).

[187] R. S. Berry and R. Breitengraser-Kunz, Phys. Rev. Lett. 74, 3951 (1995).

[188] L. Angelani, G. Parisi, G. Ruocco, and G. Viliani, Phys. Rev. Lett. 81, 4648 (1998).

[189] D. J. Wales, Energy landscapes (Cambridge University Press, Cambridge, 2003).

[190] B. Derrida, Phys. Rev. Lett. 45, 79 (1980).

[191] J. Bouchaud, Journal de Physique I 2, 1705 (1992).

[192] A. M. Samarakoon, A. Banerjee, S. Zhang, Y. Kamiya, S. E. Nagler, D. A.

Tennant, S. Lee, and C. D. Batista, Physical Review B 96, 134408 (2017). 